\title{
Experimental investigation of the vibrational and thermal response of a laser spark plug
}

\author{
Gregory S. Yoder \\ West Virginia University
}

Follow this and additional works at: https://researchrepository.wvu.edu/etd

\section{Recommended Citation}

Yoder, Gregory S., "Experimental investigation of the vibrational and thermal response of a laser spark plug" (2013). Graduate Theses, Dissertations, and Problem Reports. 3406.

https://researchrepository.wvu.edu/etd/3406

This Thesis is protected by copyright and/or related rights. It has been brought to you by the The Research Repository @ WVU with permission from the rights-holder(s). You are free to use this Thesis in any way that is permitted by the copyright and related rights legislation that applies to your use. For other uses you must obtain permission from the rights-holder(s) directly, unless additional rights are indicated by a Creative Commons license in the record and/ or on the work itself. This Thesis has been accepted for inclusion in WVU Graduate Theses, Dissertations, and Problem Reports collection by an authorized administrator of The Research Repository @ WVU. For more information, please contact researchrepository@mail.wvu.edu. 


\title{
EXPERIMENTAL INVESTIGATION OF THE VIBRATIONAL AND Thermal Response of a Laser Spark Plug
}

\author{
Gregory S. Yoder
}

\author{
Thesis submitted to the \\ College of Engineering and Mineral Resources \\ at West Virginia University \\ for partial fulfillment of the requirements \\ for the degree of
}

\author{
Masters of Science \\ in \\ Mechanical Engineering \\ Advisory Committee Members: \\ Mridul Gautam, Ph.D., Chair \\ Gregory Thompson, Ph.D. \\ Dustin McIntyre, Ph.D.
}

Department of Mechanical and Aerospace Engineering

Morgantown, West Virginia

July 26, 2013

Keywords: Laser; Ignition; Vibration; Temperature; Plasma; Combustion; Engine Copyright 2013 Gregory S. Yoder 


\section{ABSTRACT \\ EXPERIMENTAL INVESTIGATION OF THE VIBRATIONAL AND THERMAL Response of a Laser Spark Plug}

\section{Gregory S. Yoder}

A study was conducted in order to evaluate the external thermal and vibrational effects on the operation of a laser ignition system for internal combustion (IC) engine applications. West Virginia University (WVU) in conjunction with the National Energy Technology Laboratory (NETL) have constructed a prototype laser spark plug which has been designed to mount directly onto the head of a natural gas engine for the purpose of igniting an air/fuel (A/F) mixture in the engine's combustion chamber. To be considered as a viable replacement for the conventional electrode-based ignition system, integrity, durability and reliability must be justified. Thermal and oscillatory perturbations induced upon the ignition system are major influences that affect laser spark plug (LSP) operation and, therefore, quantifying these effects is necessary to further the advancement and development of this technology.

The passively q-switched Nd:YAG laser was mounted on Brüel \& Kjær (B\&K) Vibration Exciter Type 4808 Shaker in conjunction with at B\&K Power Amplifier Type 2719, which was oscillated in $10 \mathrm{~Hz}$ intervals from 0 to $60 \mathrm{~Hz}$ using a sine wave to mimic natural gas engine operation. The input signal simulated the rotational velocity of the engine operating from 0 to 3600 RPM with the laser mounted in three different axial orientations. The laser assembly was wrapped with medium-temperature heat tape, outfitted with thermocouples and heated from room temperature to $140{ }^{\circ} \mathrm{F}$ to simulate the temperatures that the LSP may experience when installed on an engine. The acceleration of the payload was varied between $50 \%$ and $100 \%$ of the oscillator's maximum allowable acceleration in each mounting orientation resulting in a total of 294 total setpoints.

For each setpoint, pulse width, pulse width variation, q-switch delay, jitter and output energy were measured and recorded. Each of these dependent variables plays a critical role in multi photon ionization and precise control is necessary to limit the variability of these key 
parameters. Under the influence of thermal and oscillatory perturbations, the q-switch delay of the laser was found to vary significantly. For application on an IC engine, such variation in qswitch delay would result in an ignition timing variation by as much as \pm 4.6 crank angle (CA) degrees in the most extreme setpoint on a cycle-to-cycle basis. Every setpoint tested was calculated to be capable of generating a plasma spark in air $\left(>100 \mathrm{GW} / \mathrm{cm}^{2}\right)$, however the resulting focal intensity was found to vary by as much as $\pm 13 \mathrm{GW} / \mathrm{cm}^{2}$. 


\section{ACKNOWLEDGEMENT}

I would like to express gratitude towards several key personal that have influenced my professional development as an engineer and have contributed towards the completion of this thesis. First and foremost, I would like to thank Mr. Dan Carder and Mr. Tom Spencer for initially giving me the opportunity to continue my educational career with WVU, and for continuously providing support and guidance for me throughout my time here. I also greatly appreciate the expertise and insight from my committee members, Dr. Mridul Gautam and Dr. Gregory Thompson.

If it were not for Dr. Azer Yalin and Dr. Sachin Joshi at CSU for initially getting me interested in the subject of laser-initiated breakdown, I would not be in the position that I am today. The training that I received from you guys at LPDL gave me the skills necessary to comprehend the physics involved with this experimentation, and the ability to repair the system when damage occurred.

I would like to acknowledge Dr. Daniel Welcome and Dr. Renguang Dong from NIOSH, who without their support would have not made a complete vibrational characterization possible. Dr Jay Wilhelm, I thank you for kindly loaning me accelerometers for the duration of this testing and for your advice throughout.

My appreciation for everything the gentlemen at NETL have done for me is unmatched. Dr. Dustin McIntyre and Dr. Steven Woodruff, I have been following your work throughout my educational career, and it has been a privilege and an honor to have worked beside you in the continued development of this revolutionary technology.

When I graduated high school, the direction for which I should apply myself was unclear. The person who exploited my potential and engaged my interest in automotive and internal combustion engine technology was Mr. Charles Watt from FRCC. You initially inspired my further education.

Finally, I would like to thank all of my friends and family who never questioned my ability of obtaining an advanced degree and who have stuck by my side throughout. 
I dedicate this thesis to my Mom. 


\section{TABLE OF CONTENTS}

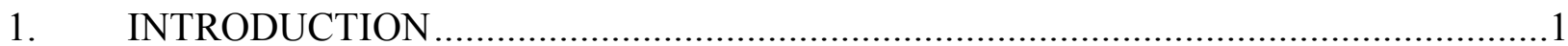

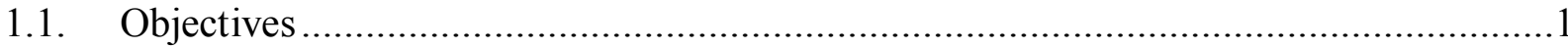

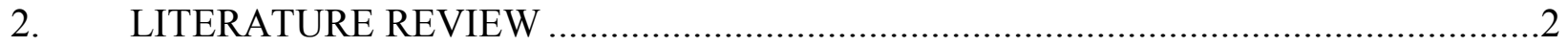

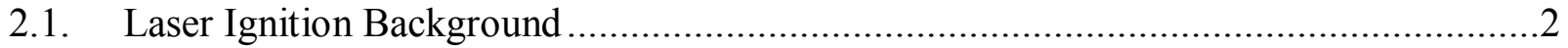

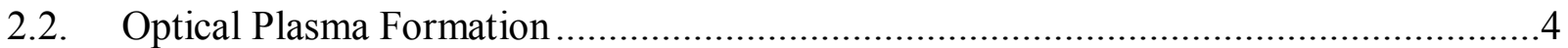

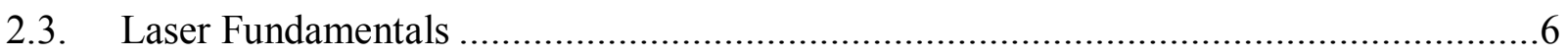

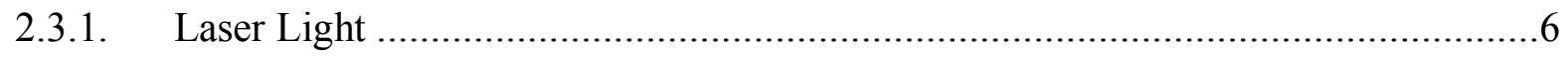

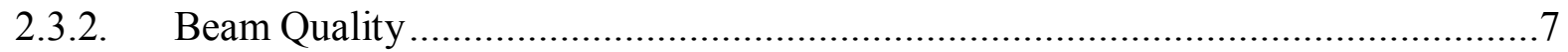

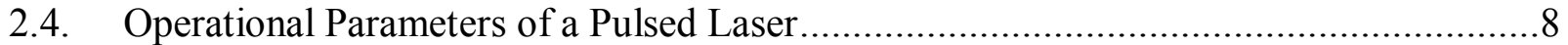

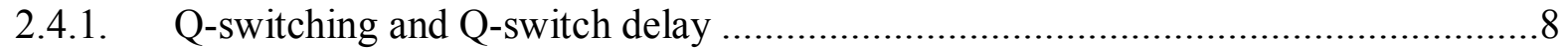

2.4.1.1. Active Q-Switching ..............................................................................12

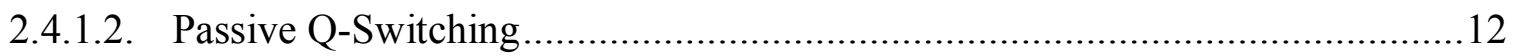

2.5. High Peak Power Delivery through Fiber Optic Cables ..............................................18

2.5.1. Multimode Solid-Core Step-Index Fibers ..........................................................19

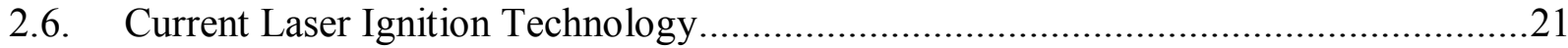

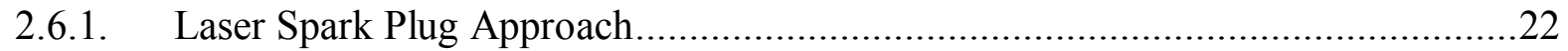

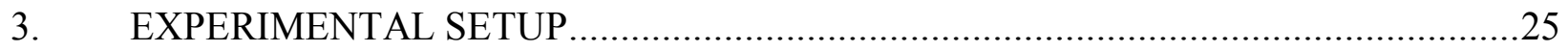

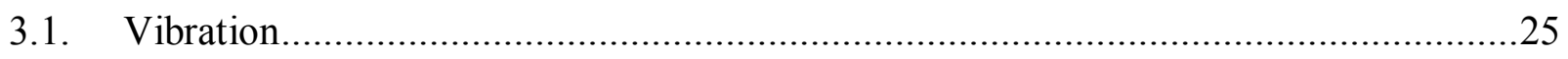

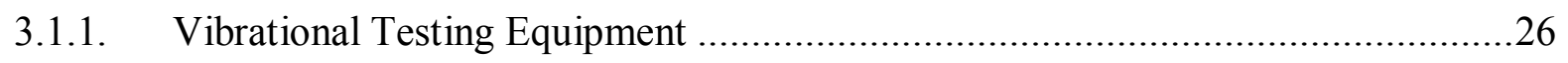

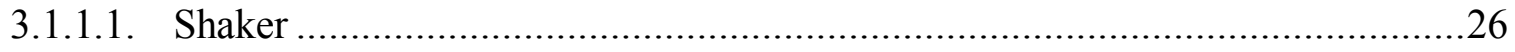

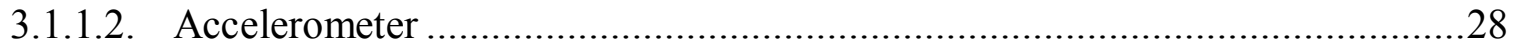

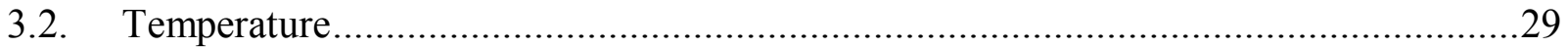

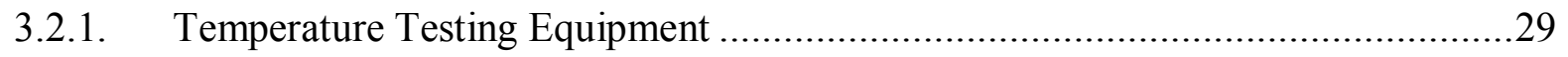

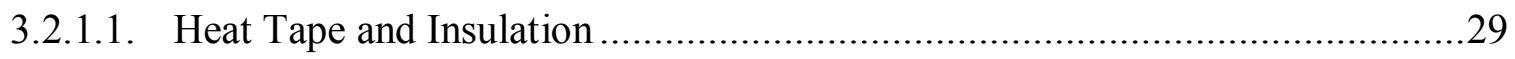

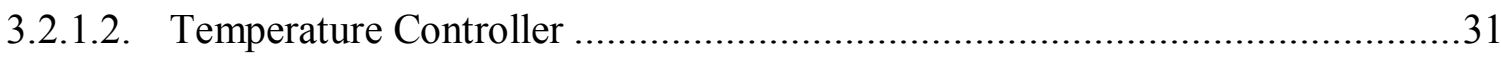

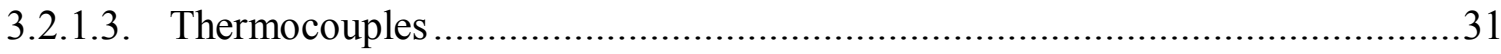

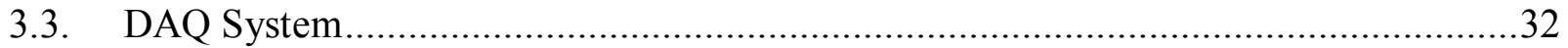

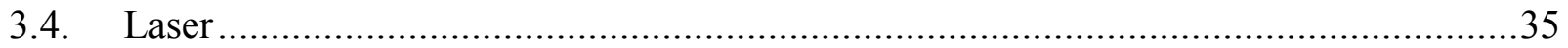

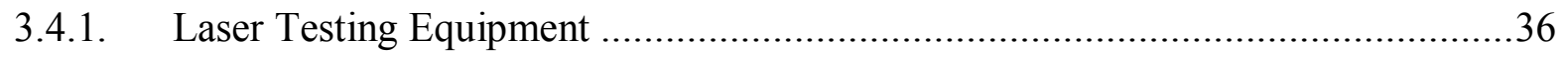

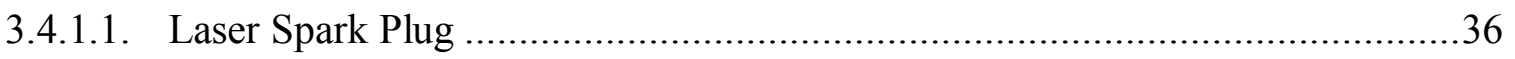

3.4.1.2. Power Meter, Oscilloscope, Detector Head and Photodiode..............................38 


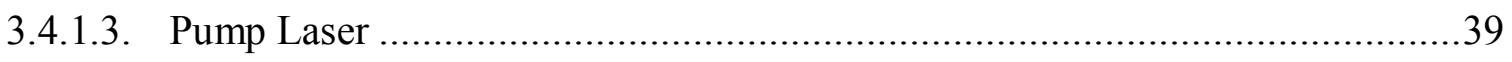

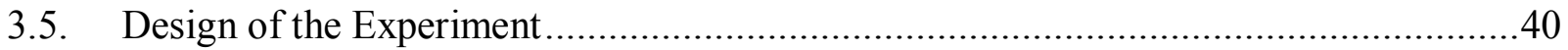

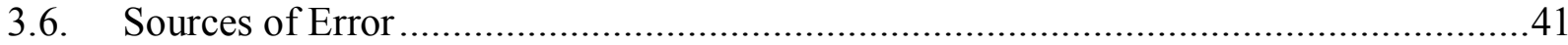

3.6.1. NI_DAQ SCB68 Shielded Desktop Connector Block..........................................41

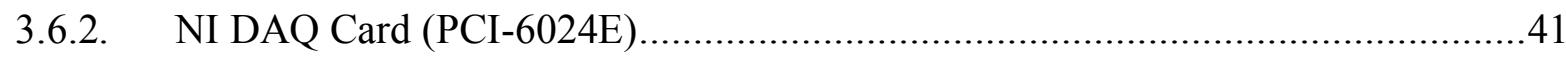

3.6.3. Omega Cement-On J-Type Thermocouples......................................................42

3.6.4. UM6 Ultra Miniature Orientation Sensor .............................................................

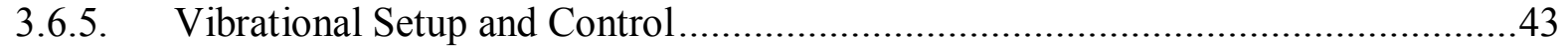

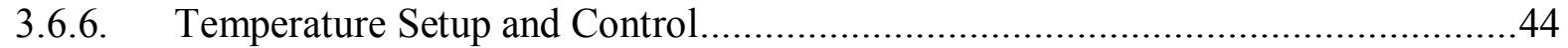

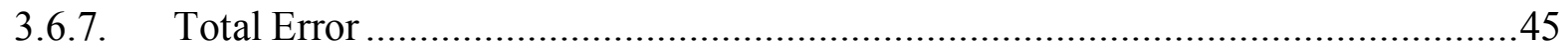

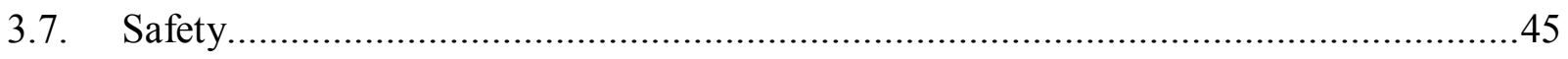

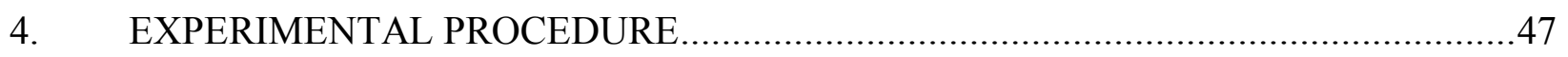

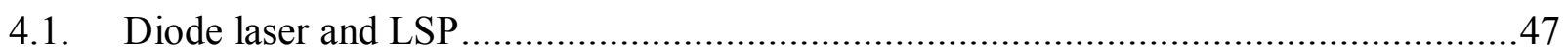

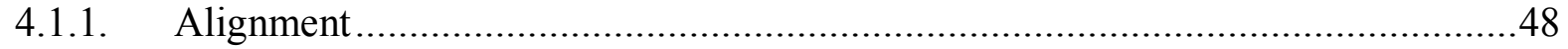

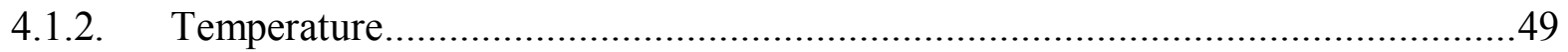

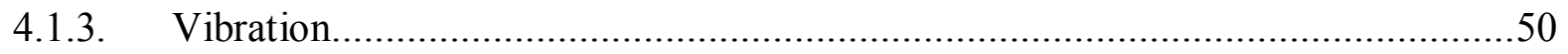

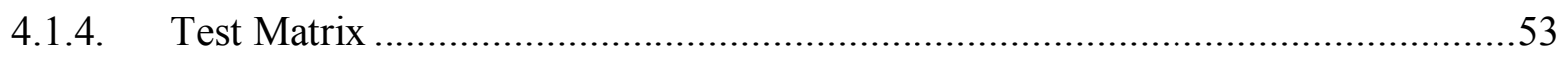

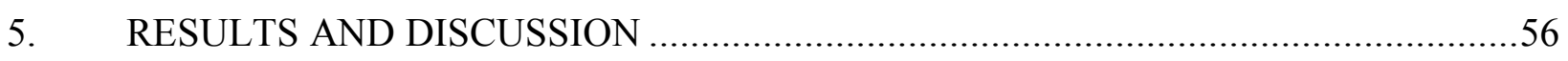

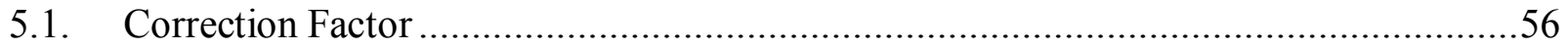

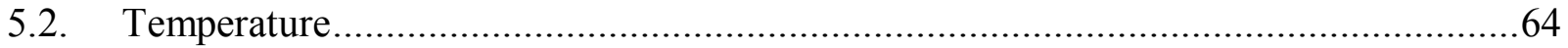

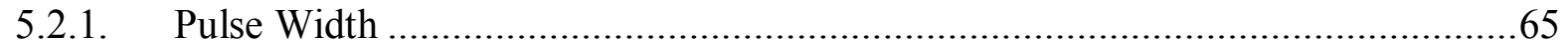

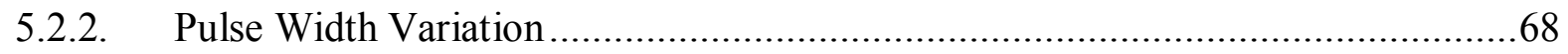

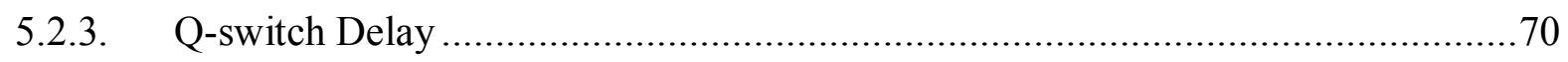

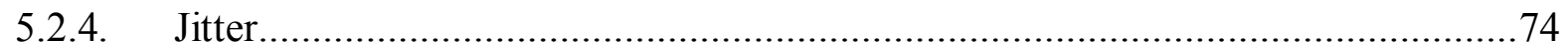

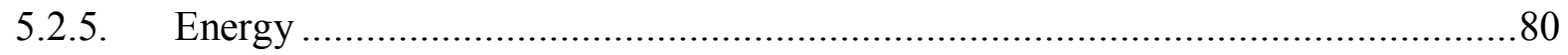

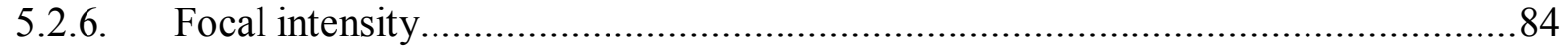

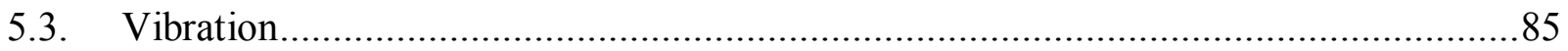

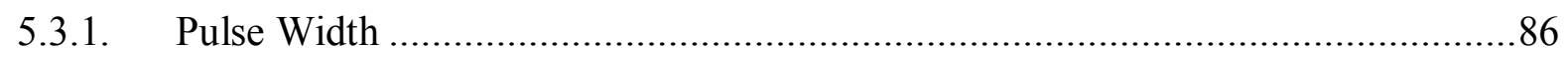

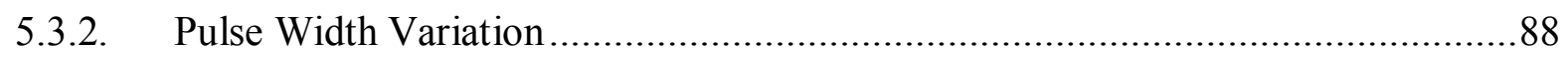

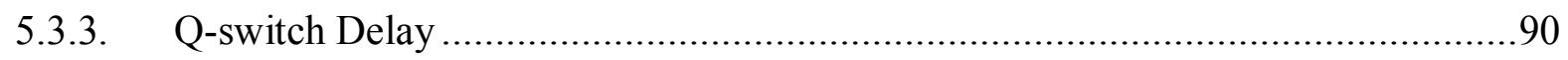

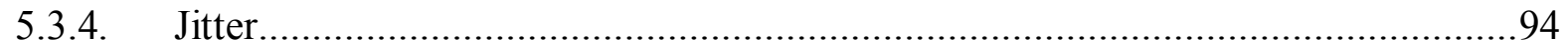

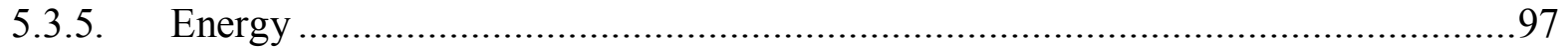




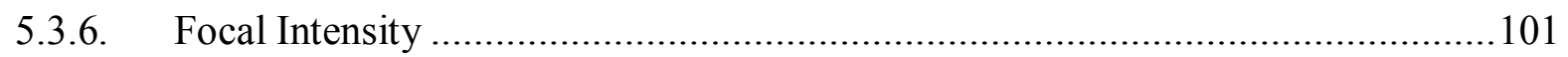

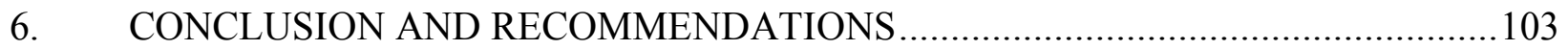

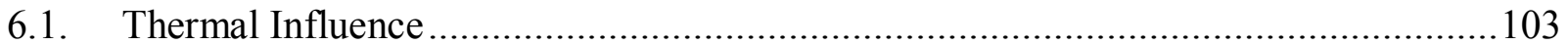

6.2. Size and Weight Distribution .................................................................... 104

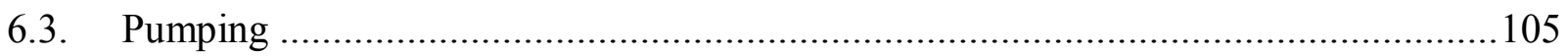

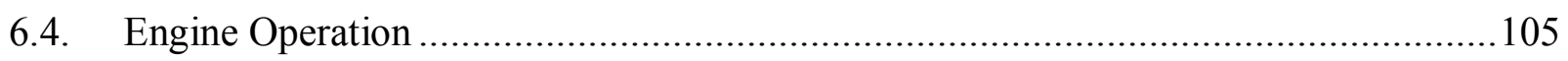

6.5. Recommendation for Future Work ............................................................. 107

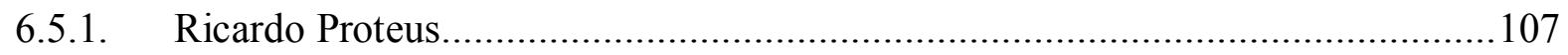

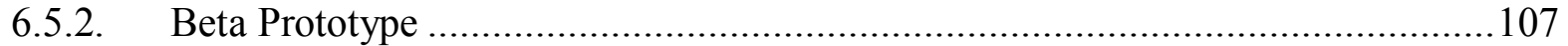

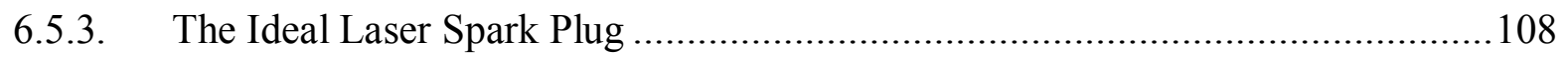

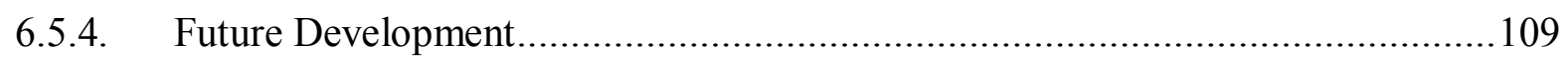

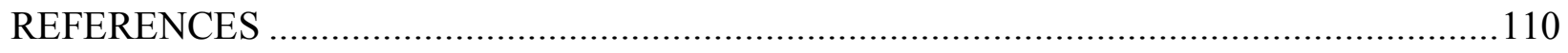

Appendix-A: Advance Ignition System Functional Requirements ................................. 115

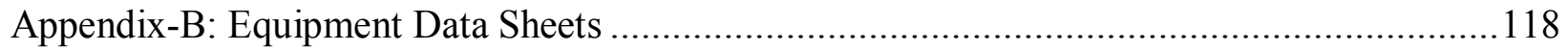

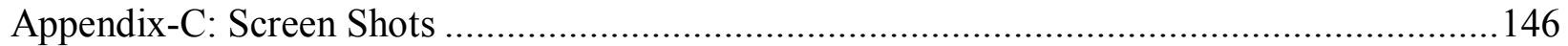

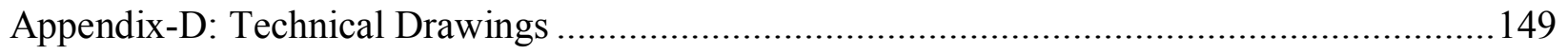

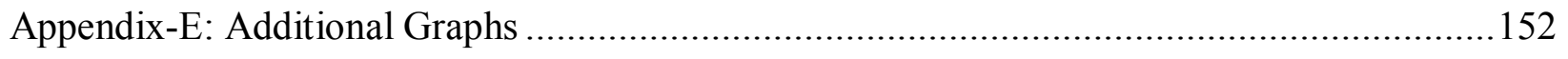

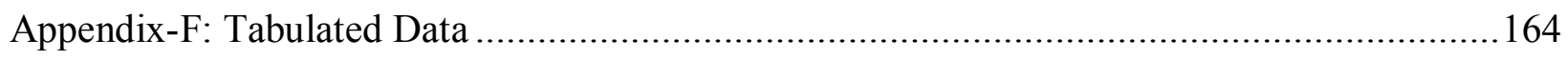




\section{LIST OF FIGURES}

Figure 1: Experimental laser breakdown thresholds of Nitrogen, Air and Methane ...................5

Figure 2: Energy level diagrams for a four level laser atomic system .................................6

Figure 3: Comparison of the focused beam waste sizes of a real laser beam and a diffraction

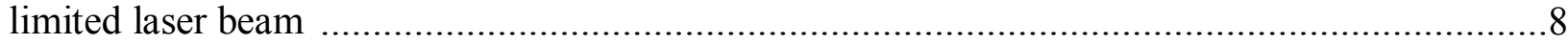

Figure 4: Pulsed energy output as a function of q-switch time delay from an actively q-switched

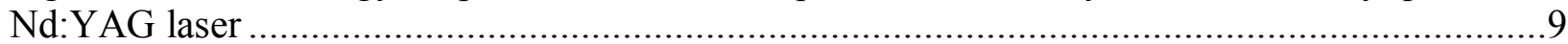

Figure 5: Measurement technique for q-switch delay using an oscilloscope ...........................10

Figure 6: CW and q-switched pulsed laser development .............................................11

Figure 7: Output power of an Nd:YAG laser as a function of input power in $\mathrm{HC}$ mode and its

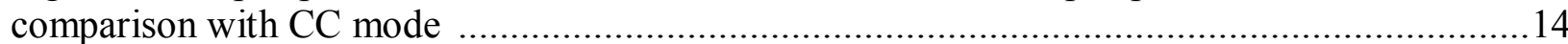

Figure 8: Initial transmission of a Cr:YAG crystal as a function of crystal temperature ...........15

Figure 9: Q-switch delay data for an end pumped laser plotted with numerical data as a function

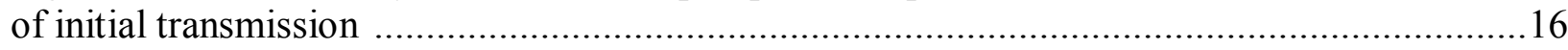

Figure 10: Calculated and measured output energy of an end pumped Nd:YAG laser .............17

Figure 11: Solid core step index fiber with exceeded damage threshold of $\sim 1-5 \mathrm{GW} / \mathrm{cm}^{2} \ldots \ldots \ldots .20$

Figure 12: Output beam intensity profile due to mode coupling from externally induced stress

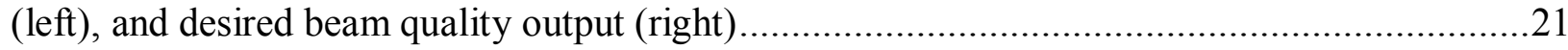

Figure 13: Strategy for multiplexing the pump energy to each respective ignition laser ..........22

Figure 14: The NETL prototype laser spark plug was initially mounted onto a research engine

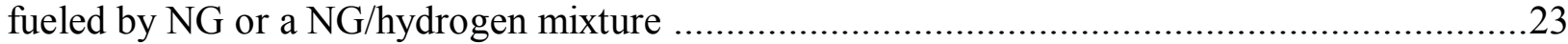

Figure 15: G-force as a function of frequency in the $\mathrm{x}$ axis for a 3.4L V6 5VZ-FE gasoline engine (left) and for a 7.3L V8 Powerstroke turbo diesel (right) ..........................................25

Figure 16: Single Axis B\&K Vibration Exciter Type 4808 Shaker .....................................26

Figure 17: Laser spark plug mounted on the vibration exciter with a $90^{\circ}$ orientation................28

Figure 18: The laser spark plug mounted in a $90^{\circ}$ orientation wrapped with the heat tape. .........30

Figure 19: Performance curves for Vibration Exciter Type 4808 for operation without assisted

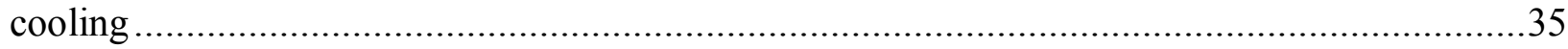

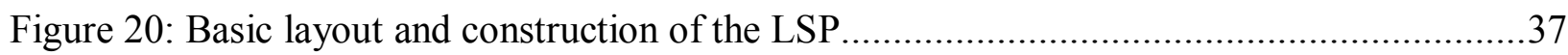

Figure 21: Positioning of the equipment in the experimental setup ....................................39

Figure 22: Calculated and actual 50\% x-axis g-force of the payload in the 90 degree mounting

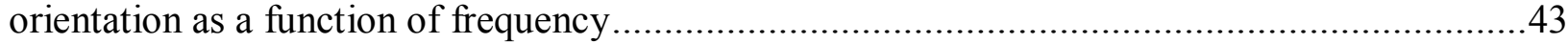

Figure 23: Laser spark plug component temperature as a function of setpoint temperature in the

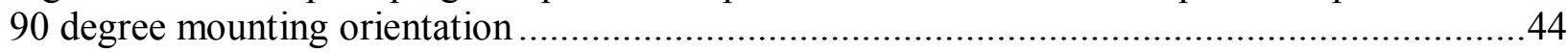

Figure 24: Danger sign posted in the optical test cell .................................................46 
Figure 25: Accelerometer mounting location for the 0 and 90 degree mounting angles..... .51

Figure 26: Generalized amplitude response of an oscillator as a function of frequency .53

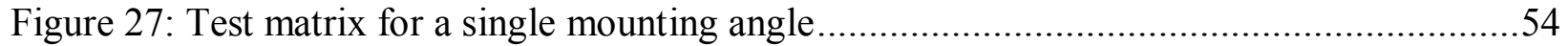

Figure 28: Four dimensional array of three cubic matrices...............................................55

Figure 29: Detector head positioning for calculation of the correction factor ..........................57

Figure 30: Average correction factor energy as a function of temperature............................59

Figure 31: Average correction factor energy as a function of pump pulse frequency ................60

Figure 32: Applied correction factor percentage as a function of temperature and pump pulse frequency.

Figure 33: Average q-switch delay as a function of pump pulse frequency in correction factor orientation.

Figure 34: Average q-switch delay as a function of heat tape temperature in correction factor orientation. .63

Figure 35: Pulse width measurement using the oscilloscope

Figure 36: Pulse width as a function of temperature for 0,45 and 90 degree mounting orientations with $100 \%$ acceleration .67

Figure 37: Pulse width as a function of temperature for the 90 degree orientation with $100 \%$ acceleration.

Figure 38: Pulse width variation as a function of temperature for 0,45 and 90 degree mounting orientations with $50 \%$ acceleration .

Figure 39: Pulse width variation as a function of temperature for the 45 degree mounting orientation with $50 \%$ acceleration

Figure 40: Average q-switch delay as a function of temperature for the 0,45 and 90 degree mounting orientations with $100 \%$ acceleration 71

Figure 41: Average q-switch delay as a function of temperature for the 45 degree mounting orientation with $100 \%$ acceleration.

Figure 42: Average q-switch delay as a function of temperature for the 90 degree mounting orientation with $100 \%$ acceleration. .73

Figure 43: Q-switch delay as a function of q-switch initial transmission. .74

Figure 44: Average jitter as a function of temperature for $50 \%$ and $100 \%$ acceleration for the 90 degree mounting orientation..... .75

Figure 45: Variation in the q-switch delay and the jitter as a function of time as observed on the oscilloscope

Figure 46: Average jitter as a function of frequency and temperature for the 90 degree mounting orientation with $100 \%$ acceleration.

Figure 47: Average jitter as a function of temperature for the 0, 45 and 90 degree mounting orientations with $100 \%$ acceleration. 
Figure 48: Jitter as a function of temperature in the 45 degree mounting orientation vibrating at

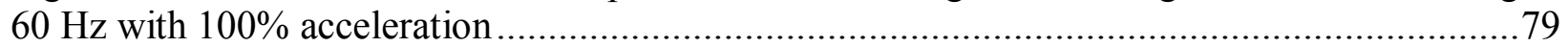

Figure 49: Jitter as a function of temperature at $60 \mathrm{~Hz}$ for the 0,45 and 90 degree mounting orientations with $100 \%$ acceleration. .80

Figure 50: Average energy as a function of temperature for 0,45 and 90 degree mounting orientations with $50 \%$ acceleration .81

Figure 51: Average energy as a function of temperature for the 45 degree mounting orientation with $50 \%$ acceleration .82

Figure 52: Average energy as a function of temperature for the 90 degree mounting orientation with $50 \%$ and $100 \%$ acceleration.. .83

Figure 53: Output energy as a function of q-switch initial transmission percentage .84

Figure 54: Focal intensity as a function of temperature for the 0, 45 and 90 degree mounting orientations with $50 \%$ acceleration . .85

Figure 55: Average pulse width as a function of frequency for the 0, 45 and 90 degree mounting orientations with $100 \%$ acceleration . .86

Figure 56: Average pulse width as a function of frequency for the 90 degree mounting angle with $100 \%$ acceleration. .87

Figure 57: Output pulse width as a function of q-switch initial transmission..... .88

Figure 58: Average pulse width variation as a function of frequency for the 0,45 and 90 degree mounting angles with $50 \%$ acceleration. . .89

Figure 59: Average pulse width variation as a function of frequency for the 45 degree mounting orientation with $50 \%$ acceleration 90

Figure 60: Average q-switch delay as a function of frequency for the 0,45 and 90 degree mounting orientations with $100 \%$ acceleration .91

Figure 61: Average q-switch delay as a function of frequency for the 90 degree mounting orientation with $100 \%$ acceleration. .92

Figure 62: Average q-switch delay as a function of frequency for the 45 degree mounting orientation with $100 \%$ acceleration. .93

Figure 63: Average q-switch delay as a function of frequency and temperature for the 90 degree mounting orientation with $100 \%$ acceleration. . .94

Figure 64: Average jitter as a function of frequency for the 90 degree mounting orientation with $50 \%$ and $100 \%$ acceleration .95

Figure 65: Average jitter as a function of frequency for the 0,45 and 90 degree mounting orientations with $100 \%$ acceleration. .96

Figure 66: Maximum jitter as a function of frequency for the 0, 45 and 90 degree mounting orientations with $100 \%$ acceleration for the most influencing temperatures .97

Figure 67: Average output energy as a function of frequency for the 0, 45 and 90 degree mounting orientations with $50 \%$ acceleration . .98 
Figure 68: Laser vibration theory for the 0 degree mounting orientation..... .99

Figure 69: Excitation regions of the Nd:YAG crystal using an end pumped strategy

Figure 70: Average output energy as a function of frequency for the 45 degree mounting orientation with $50 \%$ acceleration .100

Figure 71: Average output energy as a function of frequency for the 90 degree mounting orientation with $50 \%$ and $100 \%$ acceleration ...

Figure 72: Focal intensity as a function of frequency for the 0,45 and 90 degree mounting orientations with $50 \%$ acceleration .

Figure 73: Q-switch timing stability as a function of temperature and frequency in the 90 degree mounting orientation with $100 \%$ acceleration. 106

Figure 74: Construction of an ideal laser spark plug .

Figure 75: Q-switch delay as a function of temperature for the 0,45 and 90 degree mounting orientation with $50 \%$ acceleration 152

Figure 76: Q-switch delay as a function of frequency for the 0,45 and 90 degree mounting orientation with $50 \%$ acceleration .152

Figure 77: Pulse width variation as a function of temperature for the 0,45 and 90 degree mounting orientation with $50 \%$ acceleration.

Figure 78: Pulse width variation as a function of frequency for the 0,45 and 90 degree mounting orientation with $50 \%$ acceleration 153

Figure 79: Pulse width as a function of temperature for the 0,45 and 90 degree mounting orientation with $50 \%$ acceleration .154

Figure 80: Pulse width as a function of frequency for the 0,45 and 90 degree mounting orientation with $50 \%$ acceleration .154

Figure 81: Jitter as a function of temperature for the 0,45 and 90 degree mounting orientation with $50 \%$ acceleration .155

Figure 82: Jitter as a function of frequency for the 0,45 and 90 degree mounting orientation with $50 \%$ acceleration. .155

Figure 83: Energy as a function of temperature for the 0,45 and 90 degree mounting orientation with $50 \%$ acceleration .156

Figure 84: Energy as a function of frequency for the 0,45 and 90 degree mounting orientation with $50 \%$ acceleration . .156

Figure 85: Intensity as a function of temperature for the 0,45 and 90 degree mounting orientation with $50 \%$ acceleration .157

Figure 86: Intensity as a function of temperature for the 0,45 and 90 degree mounting orientation with $50 \%$ acceleration

Figure 87: Q-switch delay as a function of temperature for the 0, 45 and 90 degree mounting orientation with $100 \%$ acceleration. .158 
Figure 88: Q-switch delay as a function of frequency for the 0, 45 and 90 degree mounting orientation with $100 \%$ acceleration. 158

Figure 89: Pulse width variation as a function of temperature for the 0,45 and 90 degree mounting orientation with $100 \%$ acceleration

Figure 90: Pulse width variation as a function of frequency for the 0,45 and 90 degree mounting orientation with $100 \%$ acceleration.

Figure 91: Pulse width as a function of temperature for the 0,45 and 90 degree mounting orientation with $100 \%$ acceleration. 160

Figure 92: Pulse width as a function of frequency for the 0,45 and 90 degree mounting orientation with $100 \%$ acceleration 160

Figure 93: Jitter as a function of temperature for the 0,45 and 90 degree mounting orientation with $100 \%$ acceleration 161

Figure 94: Jitter as a function of frequency for the 0,45 and 90 degree mounting orientation with $100 \%$ acceleration 161

Figure 95: Energy as a function of temperature for the 0,45 and 90 degree mounting orientations with $100 \%$ acceleration 162

Figure 96: Energy as a function of frequency for the 0, 45 and 90 degree mounting orientations with $100 \%$ acceleration 162

Figure 97: Intensity as a function of temperature for the 0,45 and 90 degree mounting orientations with $100 \%$ acceleration 163

Figure 98: Intensity as a function of frequency for the 0, 45 and 90 degree mounting orientations with $100 \%$ acceleration 163 


\section{LIST OF TABLES}

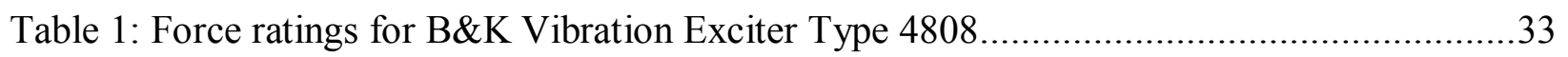

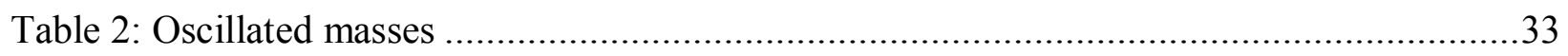

Table 3: Maximum acceleration and g-force calculations for every combination of testing

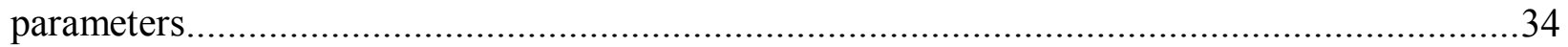

Table 4: Pump pulse width used for each thermal and mounting orientation setpoint ...............47

Table 5: Amplifier gain needed to reach maximum (100\%) g-force of the payload for each

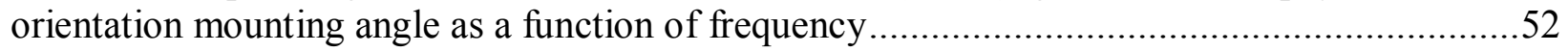

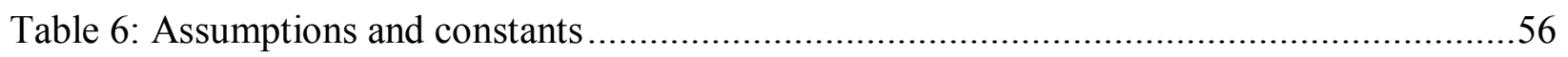

Table 7: Pulse width (ns) in the 0 degree mounting orientation with 50\% acceleration ...........164

Table 8: Pulse width (ns) in the 0 degree mounting orientation with $100 \%$ acceleration ..........164

Table 9: Pulse width (ns) in the 45 degree mounting orientation with 50\% acceleration ..........165

Table 10: Pulse width (ns) in the 45 degree mounting orientation with $100 \%$ acceleration.......165

Table 11: Pulse width (ns) in the 90 degree mounting orientation with 50\% acceleration.........166

Table 12: Pulse width (ns) in the 90 degree mounting orientation with $100 \%$ acceleration.......166

Table 13: Pulse width variation (ns) in the 0 degree mounting orientation with $50 \%$ acceleration

Table 14: Pulse width variation (ns) in the 0 degree mounting orientation with $100 \%$ acceleration .167

Table 15: Pulse width variation (ns) in the 45 degree mounting orientation with $50 \%$ acceleration .168

Table 16: Pulse width variation (ns) in the 45 degree mounting orientation with 100\% acceleration. .168

Table 17: Pulse width variation (ns) in the 90 degree mounting orientation with $50 \%$ acceleration .169

Table 18: Pulse width variation (ns) in the 90 degree mounting orientation with 100\% acceleration. 169

Table 19: Q-switch delay ( $\mu \mathrm{s})$ in the 0 degree mounting orientation with $50 \%$ acceleration ..... 170 Table 20: Q-switch delay ( $\mu \mathrm{s})$ in the 0 degree mounting orientation with $100 \%$ acceleration ... 170 Table 21: Q-switch delay ( $\mu \mathrm{s})$ in the 45 degree mounting orientation with $50 \%$ acceleration ... 171 Table 22: Q-switch delay ( $\mu \mathrm{s})$ in the 45 degree mounting orientation with 100\% acceleration .171 Table 23: Q-switch delay ( $\mu \mathrm{s})$ in the 90 degree mounting orientation with $50 \%$ acceleration ... 172 Table 24: Q-switch delay ( $\mu \mathrm{s})$ in the 90 degree mounting orientation with $100 \%$ acceleration .172 Table 25: Jitter $(\mu \mathrm{s})$ in the 0 degree mounting orientation with $50 \%$ acceleration. 173 
Table 26: Jitter $(\mu \mathrm{s})$ in the 0 degree mounting orientation with $100 \%$ acceleration..................173

Table 27: Jitter $(\mu \mathrm{s})$ in the 45 degree mounting orientation with $50 \%$ acceleration..................174

Table 28: Jitter $(\mu \mathrm{s})$ in the 45 degree mounting orientation with $100 \%$ acceleration................174

Table 29: Jitter $(\mu \mathrm{s})$ in the 90 degree mounting orientation with $50 \%$ acceleration..................175

Table 30: Jitter $(\mu \mathrm{s})$ in the 90 degree mounting orientation with $100 \%$ acceleration................175

Table 31: Energy $(\mathrm{J})$ in the 0 degree mounting orientation with 50\% acceleration ..................176

Table 32: Energy $(\mathrm{J})$ in the 0 degree mounting orientation with $100 \%$ acceleration ................176

Table 33: Energy $(\mathrm{J})$ in the 45 degree mounting orientation with 50\% acceleration ................177

Table 34: Energy $(\mathrm{J})$ in the 45 degree mounting orientation with 100\% acceleration...............177

Table 35: Energy $(\mathrm{J})$ in the 90 degree mounting orientation with $50 \%$ acceleration ................178

Table 36: Energy $(\mathrm{J})$ in the 90 degree mounting orientation with $100 \%$ acceleration...............178

Table 37: Focal intensity $\left(\mathrm{GW} / \mathrm{cm}^{2}\right)$ in the 0 degree mounting orientation with $50 \%$ acceleration 179

Table 38: Focal intensity $\left(\mathrm{GW} / \mathrm{cm}^{2}\right)$ in the 0 degree mounting orientation with $100 \%$ acceleration 179

Table 39: Focal intensity $\left(\mathrm{GW} / \mathrm{cm}^{2}\right)$ in the 45 degree mounting orientation with $50 \%$ acceleration 180

Table 40: Focal intensity $\left(\mathrm{GW} / \mathrm{cm}^{2}\right)$ in the 45 degree mounting orientation with $100 \%$ acceleration.

Table 41: Focal intensity $\left(\mathrm{GW} / \mathrm{cm}^{2}\right)$ in the 90 degree mounting orientation with $50 \%$ acceleration 181

Table 42: Focal intensity $\left(\mathrm{GW} / \mathrm{cm}^{2}\right)$ in the 90 degree mounting orientation with $100 \%$ acceleration 


\section{NOMENCLATURE}

$\mathrm{A} / \mathrm{F}$

ANSI

$\mathrm{B} \& \mathrm{~K}$

CA

CAFEE

$\mathrm{CC}$

CCD

CFR

CHR

Cr:YAG

$\mathrm{CW}$

DAQ

DOE

DVD

EMF

FWHM

$\mathrm{HC}$

IC

$\mathrm{I} / \mathrm{O}$

IR

LASER

LI

LLNL

LSP

$\mathrm{M}^{2}$

Nd:YAG

NETL

NI

$\mathrm{NIOSH}$

NIST

NOx
-Air to fuel equivalence ratio

-American National Standards Institute

-Brüel \& Kjær

-Crank angle

-Center for Alternative Fuels Engines and Emissions

-Continuous cooling

-Carbon chamber deposition

-Code of Federal Regulations

-CH Robotics

-Chromium-doped yttrium aluminum garnet $\left(\mathrm{Cr}^{4+}: \mathrm{Y}_{3} \mathrm{Al}_{5} \mathrm{G}_{12}\right)$

-Continuous wave

-Data acquisition

-Department of Energy

-Digital video disk

-Electromotive force

-Full width half maximum

-Heat capacity

-Internal combustion

-Input or output

-Infrared

-Light amplification by stimulated emission of radiation

-Laser ignition

-Lawrence Livermore National Laboratory

-Laser spark plug

-Laser beam quality

-Neodymium-doped yttrium aluminum garnet $\left(\mathrm{Nd}^{3+}: \mathrm{Y}_{3} \mathrm{Al}_{5} \mathrm{G}_{12}\right)$

-National Energy Technology Laboratory

-National Instruments

-National Institute for Occupational Safety and Health

-National Institute of Standards and Technology

-Oxides of nitrogen 
OSHA

PCX

PID

PPE

Q

$\mathrm{R}^{2}$

RPM

SI

SMA

SOP

TEM

TDS

TTL

USB

VI

WV

WVU
-Occupational Safety and Health Administration

-Plano-convex lens

-Proportional integral derivative

-Personal protection equipment

-Quality

-Coefficient of determination

-Revolutions per minute

-Spark ignition

-Subminiature version A

-Standard operating procedure

-Transverse electromagnetic mode

-Tektronix Distribution Services

-Transistor-transistor logic

-Universal serial bus

-Virtual image

-West Virginia

-West Virginia University 


\section{INTRODUCTION}

To improve the combustion process in a natural gas fueled internal combustion (IC) engine, an alternative ignition source was considered. The use of a laser to ignite an air/fuel (A/F) mixture has been proven to be a viable technology over the conventional spark ignition (SI) system and has the potential to improve several facets of engine performance including brake thermal and fuel efficiency, exhaust constituent emissions and power output. By use of a high peak power solid-state laser and a fiber-coupled pumping source, an ignition system is realized for the spatially complex, high energy pulse transfer necessary for plasma spark generation.

The progress of laser ignition (LI) has yet to develop to the point where it may be deemed applicable for industrial use primarily because durability, integrity and reliability are of major concern. The implementation of such a sensitive optical system into a harsh engine environment presents a multitude of challenges that must be overcome prior to becoming a suitable replacement for the conventional electrode-based ignition system. Limitations set forth by the application, such as the influence of temperature and vibration, present obstacles regardless of the engine to which the alternative ignition source is being applied. The purpose of this study was to expose these limitations, and to characterize the effects that temperature and vibration will have on the LI system's performance.

\subsection{Objectives}

The global objective of this study was to promote the advancement of LI systems for natural gas engine applications. The primary objective was to study the effect that temperature and vibration have on the operation of the laser spark plug (LSP). The specific objectives are (1) assess the effect that temperature and vibration each had on the output characteristics of the LSP, (2) assess the combined effect that temperature and vibration have on the LSP, and (3) present the collected results in such a manner as to be beneficial towards the continued research and development of a safe, durable and marketable alternative ignition source. 


\section{LITERATURE REVIEW}

\subsection{Laser Ignition Background}

As emission regulations continue to become more stringent for IC engines, optimization of the in-cylinder combustion process is becoming more critical. The movement to reduce the amount of harmful engine emissions entering our atmosphere begins with perfecting the ignition strategy of the engine. The conventional electrode-based spark ignition strategy has been widely utilized to initiate combustion in a multitude of SI engines, however it is fundamentally limited for igniting natural gas in an IC engine and an alternative means of igniting the A/F mixture may prove to yield better combustion efficiency.

The use of a laser to ignite $\mathrm{A} / \mathrm{F}$ mixtures in an IC engine is a comparatively young technology, compared to conventional electrode-based spark ignited method, and possesses great potential to advance the IC engine industry. Typically, a laser is q-switched to produce a high peak power pulsed beam, which with the proper accompanying optics, has the capability of producing a plasma spark. Chen, a plasma physicist, defines plasma as, "A quasineutral gas of charged and neutral particles, which exhibits collective behavior" [1]. This phenomena has been applied in several different LI experimental setups such as open air beam transfer [2, 3], transmission via fiber optic cables $[4,5,6,7,8,9]$ or a single laser mounted directly onto the head of each cylinder $[10,11]$. Depending on the limitations set forth by the application, one of these LI strategies may provide certain advantages for initiating combustion over the others.

An alternative ignition source, such as LI, offers several advantages over a conventional spark plug. Unlike a spark plug where the point of ignition is limited to the top of the combustion chamber near the head, a laser with the proper accompanying optics has the capability to produce a plasma spark virtually anywhere in the combustion chamber, allowing for optimization of the combustion process $[4,5,10,11,12]$. This allows for more complete combustion to be achieved, as the spark location may be optimized to best conform to the geometry of the engine, resulting in an extension of lean limit operation $[2,4,5,10,13]$. Even with the application of current strategies to optimize the combustion process, carbon chamber deposition (CCD) is an unfortunate byproduct which leads to fouling of the conventional spark plug electrodes for several gaseous and liquid fuels. In the case of clean fuels such as natural gas however, the effects from CCD are not significant. With the use of a non-invasive LI ignition 
source, particle deposition now has a minimal influence on ignition characteristics. It has been shown that as a laser pulse passes through the optically accessible quartz window in the combustion chamber, the particles that have accumulated on the window from the previous combustion event undergo an ablation process where the deposits may partially absorb the laser pulse, heated to the point of evaporation and are removed from the window [14, 15]. This is a key advantage over the conventional system in that this ignition source is self-maintaining.

The use of a plasma spark is rather attractive for pressurized applications as well, due to the fact that a combustible medium's optical breakdown threshold decreases with increasing pressure $[16,17]$. These ignition features allow for an engine to operate at a higher compression ratio and leaner $\mathrm{A} / \mathrm{F}$ mixture providing greater fuel efficiency, reduced emissions and a more complete combustion process than is capable with the use of an electrode-based spark plug [2, 3, $4,5,10,11,12,13]$.

The reduction of NOx formation is the primary reason a natural gas engine would be operated with a lean $\mathrm{A} / \mathrm{F}$ ratio. If a mixture is too lean, a misfire event can occur resulting in improper engine operation. To compensate for this, the compression ratio of the engine may be increased. A higher compression ratio will result in higher cylinder pressures which becomes problematic for electrode-based spark plugs. At elevated pressures, a higher voltage potential is required to breakdown the plug's gap to create the spark. This abides by Paschen's law which states that as the pressure of a gas is increased, it takes a higher potential difference to initiate the electrical breakdown of the gas [18]. As pressure is increased in the engine's combustion chamber, there is a higher quantity of air and fuel molecules being squeezed into the volume in between the spark plug electrodes. This necessitates a higher amount of ignition voltage to jump the electrode gap.

Since more current is needed to pass through the electrodes to initiate and sustain breakdown at elevated pressures, the spark plug electrodes wear out quicker requiring a higher frequency of replacement. Spark plug degradation is a strong argument for utilizing a laser to initiate ignition rather than a conventional spark plug. For engine owners, this degradation is unattractive since a higher replacement frequency means a higher out of pocket cost for engine maintenance as well as the loss from the downtime of the engine. Therefore an alternative means of ignition, such as a laser spark, is desirable in these operating conditions. 


\subsection{Optical Plasma Formation}

There are a total of four physical processes whereby a laser can be used to induce breakdown in gaseous mixtures: thermal heating, photochemical excitation, resonant breakdown, and non-resonant breakdown [19]. The dominant mechanism used in this and several other LI strategies is non-resonant breakdown [2]. Non-resonant breakdown is a process in which initial electrons absorb the energy from the laser pulse via the inverse Bremsstrahlung process whose exact mechanism is further defined in the literature [20,21].

The optical breakdown location within the cylinder can be controlled via the focal length of the focusing lens and the steering optics. This versatility allows for the combustion process to be optimized based on the engine geometry and fuel delivery strategy to provide for a more complete and uniform burn to occur, leading to higher fuel efficiency and a greater power output [8]. The range of focal lengths which can be applied to the system is fundamentally bounded by the power and beam quality considerations of the system. A longer focal length results in a larger spot size at the focal point of the lens requiring a greater laser power to initiate the formation of plasma [22].

Focusing a high power laser pulse produces a high optical intensity at the focal point of the lens. If this local intensity is greater than the breakdown voltage of a given medium, multiphoton ionization will occur and release electrons onto the medium [23]. These new free electrons are accelerated by the electric field produced by the photons and collide with other atoms of the given medium. This further ionizes the atoms leading to an avalanche cascade of electron release, referred to in this work as a plasma spark. At standard atmospheric pressure and temperature, the breakdown intensity of air for plasma formation is approximately 100-200 $\mathrm{GW} / \mathrm{cm}^{2}$ [8]. A laser spark is desirable in spark ignition applications due to the decrease of a gaseous medium's breakdown intensity with increasing pressure [8].

As pressure is increased in the combustion chamber, there are more insulators that are being squeezed into the focal volume of the LSP. As previously discussed, plasma formation generated from a laser source works on the principal of molecules absorbing the energy from the laser, thereby increasing the energy of the molecules and thus leading to the subsequent release of electrons as the breakdown threshold is reached [8]. In a high pressure atmosphere, breakdown is more readily achieved due to the fact that there are more molecules in the focal volume to absorb the laser's energy. More efficient energy transfer to the gas occurs at higher 
pressures. This is a primary advantage of LI over the electrode-based spark plug. Figure 1 shows this trend in breakdown threshold intensities for some common gasses as a function of pressure.

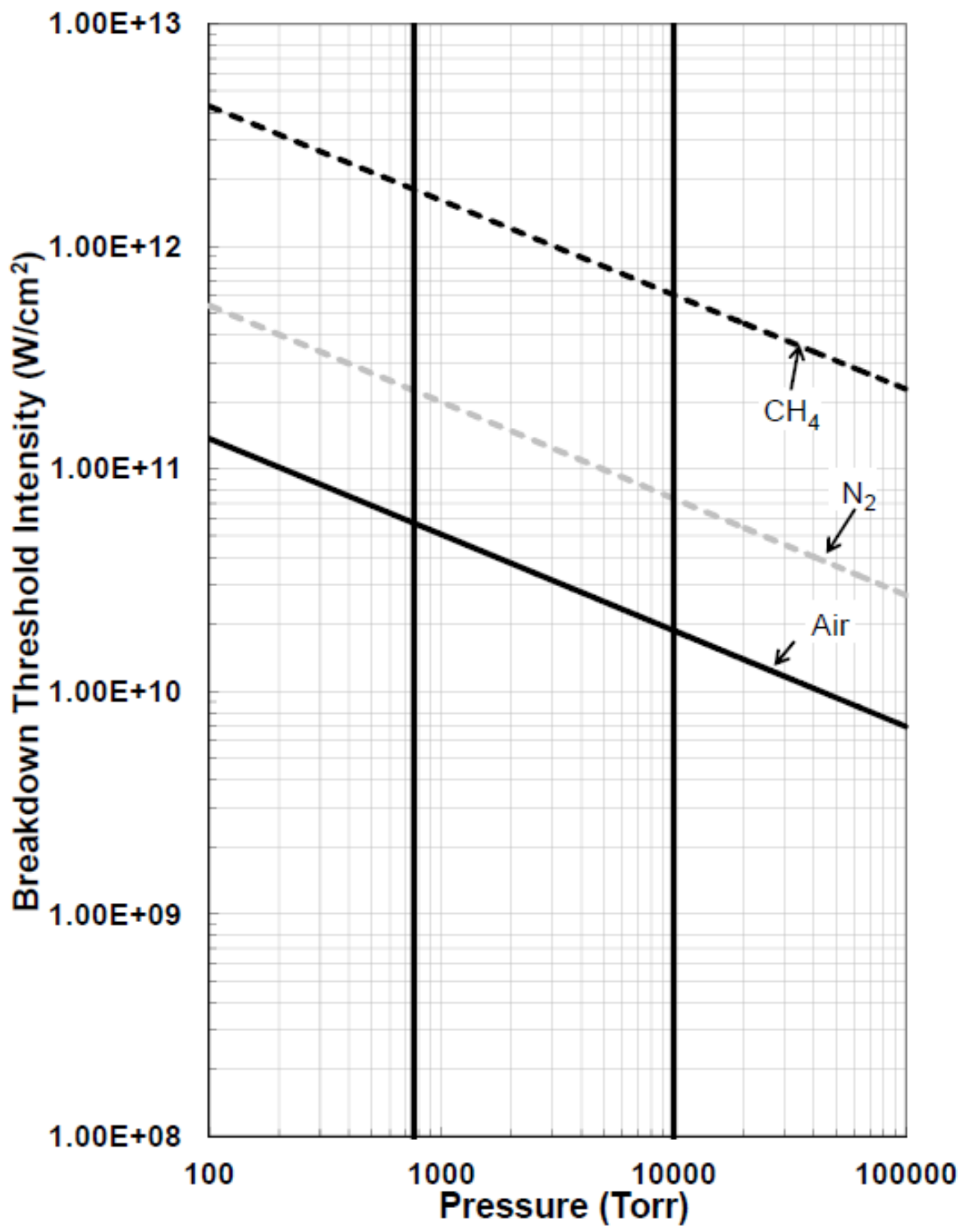

Figure 1: Experimental laser breakdown thresholds of Nitrogen, Air and Methane [21] 


\subsection{Laser Fundamentals}

\subsubsection{Laser Light}

The acronym LASER stands for Light Amplification by Stimulated Emission of Radiation. In order to stimulate an excited state in a laser gain medium, a means of transferring pump energy to the media is required. For a particular gain media, the active elements of the material require a pumping mechanism capable of exciting the elements electrons an appropriate energy level. Injection of energy creates a large collection of atoms in their excited state. This causes what is known as a population inversion as there are more excited atoms than atoms at the ground state. Once the media's electrons have reached this higher energy level, they naturally want to return to their ground state to satisfy the equilibrium in their respective atom. By doing so, energy in the form of photons is released as media's electrons relax to lower energy states. This natural photon release is monochromatic whose wavelength is dependent upon the properties of the excitation media. When two atoms of identical composition contain electrons dropping from the same higher energy level to the same lower energy level, photons of identical wavelengths will be released. The classical energy level diagram for a four level atomic laser system is shown below in Figure 2.
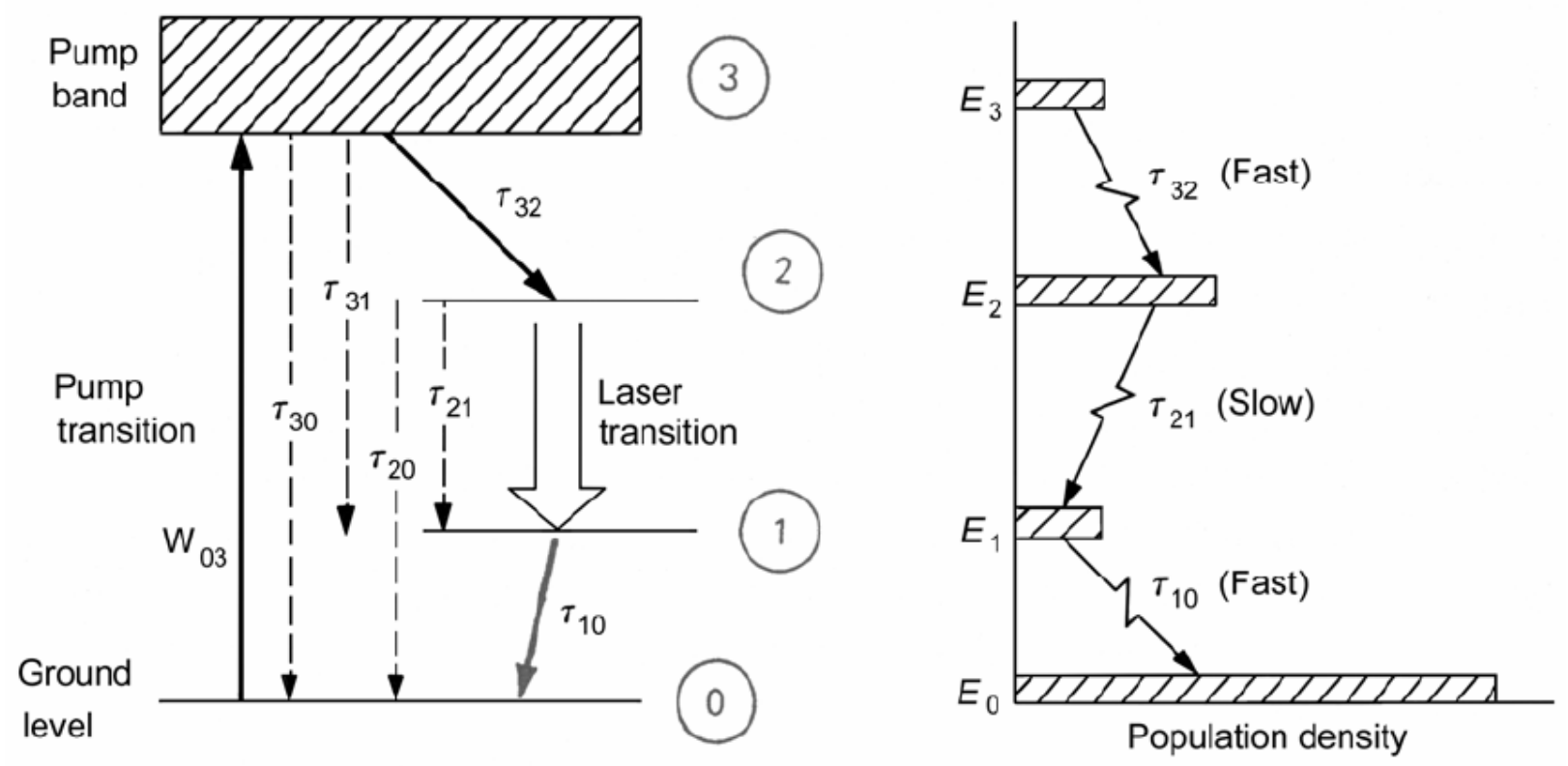

Figure 2: Energy level diagrams for a four level laser atomic system [24] 
As photons are emitted, they get trapped in the optical resonator and travel back and forth through the lasing medium where they interact with other atoms in their excited state. This process induces stimulated emission causing photons of the same phase, wavelength and direction of travel to be emitted. An exponential increase of stimulated emission then occurs allowing for the generation of a large amount of photons in the resonator. Some photons from the resonating cavity are allowed to leak through the output coupler (OC) and the resulting output is a directional, coherent, monochromatic, continuous wave laser beam.

\subsubsection{Beam Quality}

The quality of the output beam is quantified by its $\mathrm{M}^{2}$ value and dependent upon the characteristics of the beam. It is the ratio of the actual beam parameters over the ideal Gaussian beam $\left(\mathrm{TEM}_{00}\right)$ where the best possible beam quality has $\mathrm{M}^{2}=1$ [25]. Equation 2-1 shows the calculation for the diffraction limited spot diameter that can be obtained for a given lens at a given wavelength for a given output beam diameter. Here, $d_{0}$ is known as the diffraction limited spot diameter and it represents the beam diameter at the focal point of a lens assuming a $\mathrm{M}^{2}=1$ for a perfectly Gaussian beam.

$d_{0}=\frac{4 \lambda f_{L}}{\pi D}$

The parameter $D$ is the diameter of the laser beam at the input of the focusing lens, $f_{L}$ is the focal length of the focusing lens and $\lambda$ is the operating wavelength of the laser. The $M^{2}$ factor relates the diffraction limited spot diameter to the actual spot diameter that will be achieved by an imperfect beam being focused through an imperfect lens. The factor is simply multiplied by the diffraction limited beam diameter to obtain the real spot diameter $D_{m}$ as shown in Equation $2-2$.

$D_{m}=M^{2} d_{0}$

The relationship between focusing an actual laser beam and focusing a perfect diffraction limited laser beam is graphically shown below in Figure 3. Here it is visually shown that a much tighter focal diameter can be achieved with higher beam quality. The diameter of the focal spot size affects the focal intensity. As $D_{m}$ increases, the focal intensity decreases. This relationship is shown in Equation 2-3 where $I_{m}$ is the focal intensity and $P$ is the laser output power. 
$I_{m}=\frac{4 P}{\pi D_{m}}$

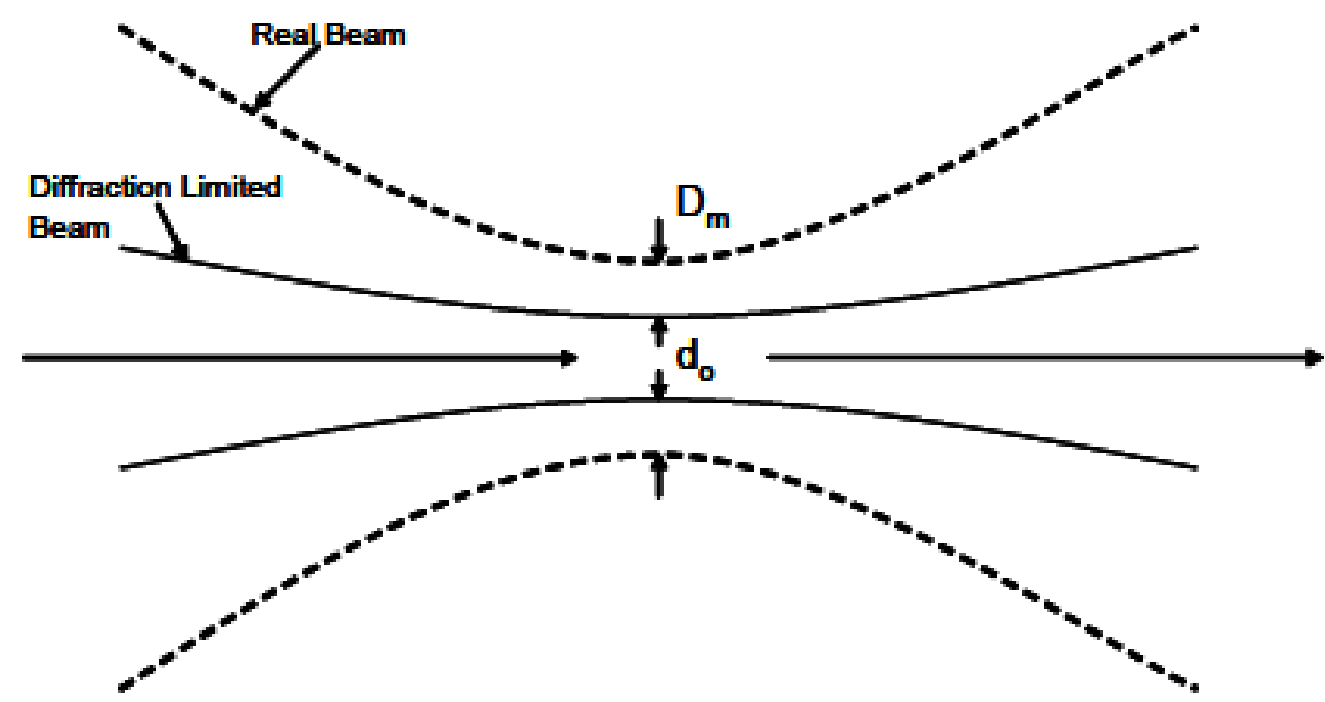

Figure 3: Comparison of the focused beam waste sizes of a real laser beam and a diffraction limited laser beam [21]

The quality of the output beam is an important factor in LI applications. The closer the $\mathrm{M}^{2}$ gets to 1 , the lower the power requirements are to generate a laser spark. A beam with low $\mathrm{M}^{2}$ has a uniform energy distribution allowing for a uniform energy distribution at the focal point. This is desired in LI applications since the better the $\mathrm{M}^{2}$ the less amount of energy is required to achieve multiphoton ionization. It is still possible to generate a plasma spark using a beam of poor quality however it takes considerably more energy.

\subsection{Operational Parameters of a Pulsed Laser}

\subsubsection{Q-switching and Q-switch delay}

Q-switching is an optical technique used for obtaining short energetic pulses from a laser by means of modulating the intracavity losses. Energy from a diode laser is directed into the LSP's gain media where it is used to produce an excited state population inversion. When the gain medium has reached a certain level of excited state population inversion, the energy is dumped from the cavity resulting in a high energy pulse of light in a short amount of time. This energy pulse is accomplished with the use of a q-switch. It is essentially an optical gate that 
allows for an energy potential to build to a desired level and to be quickly dumped once the energy has reached this desired level.

Q-switch delay is an important parameter that affects the timing of the laser output pulse. Figure 4 shows how the output pulse energy will vary as a function of the q-switch delay. Too long of time delay between the pump pulse and the bleaching of the q-switch will result a decrease in the population inversion within the cavity. Too short of a time delay will result in a reduction in output energy as well since the population inversion did not have a sufficient amount of time to reach its maximum value [26].

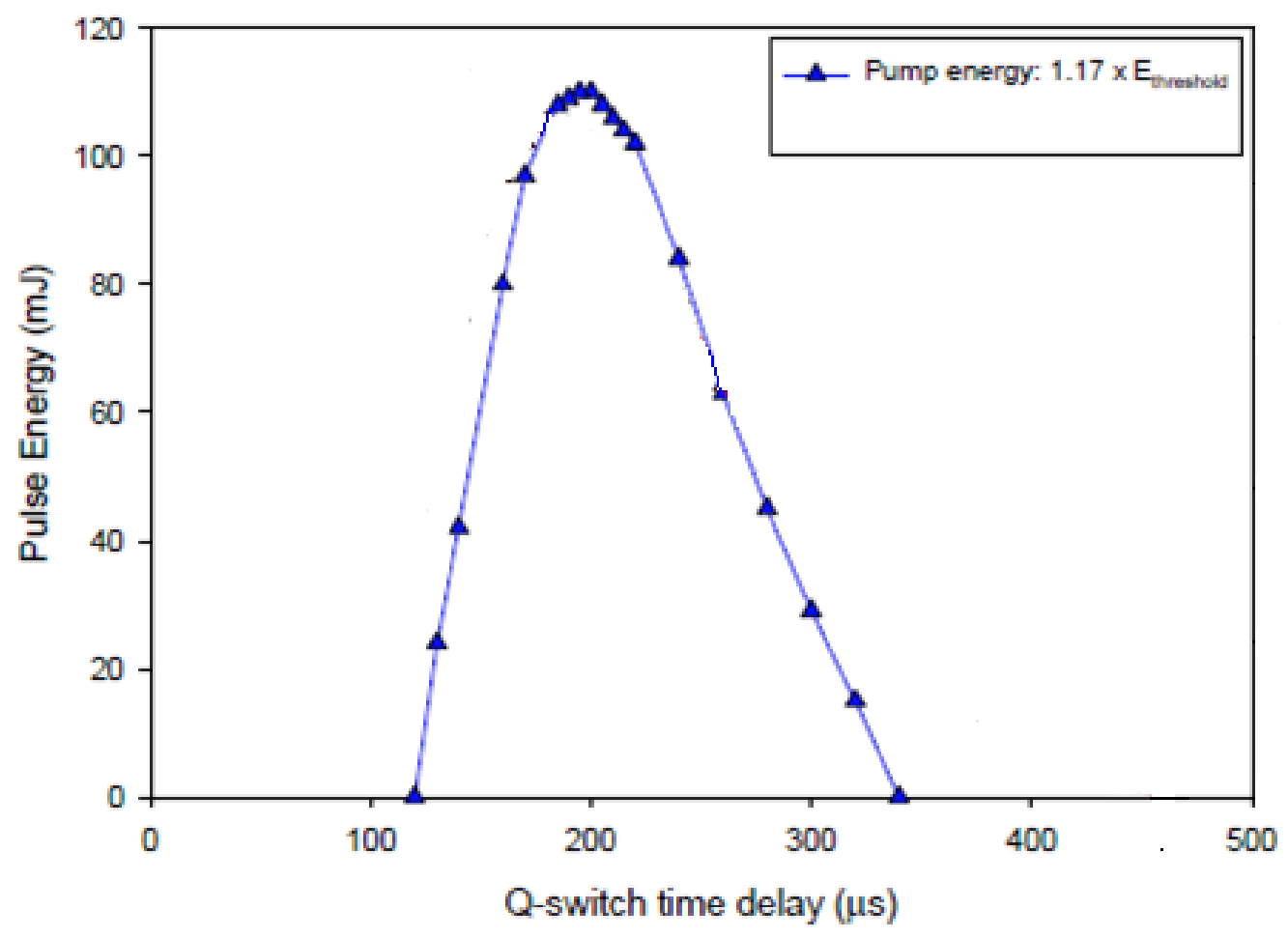

Figure 4: Pulsed energy output as a function of q-switch time delay from an actively q-switched Nd:YAG laser [26]

The q-switch delay is defined as the time from when the pump energy enters the cavity to the point where the q-switch becomes saturated and laser output is produced [26]. The q-switch delay can be quantified with the use of an oscilloscope as shown in Figure 5. The q-switch delay is a function of pump energy, resonant cavity material parameters and environmental conditions. 


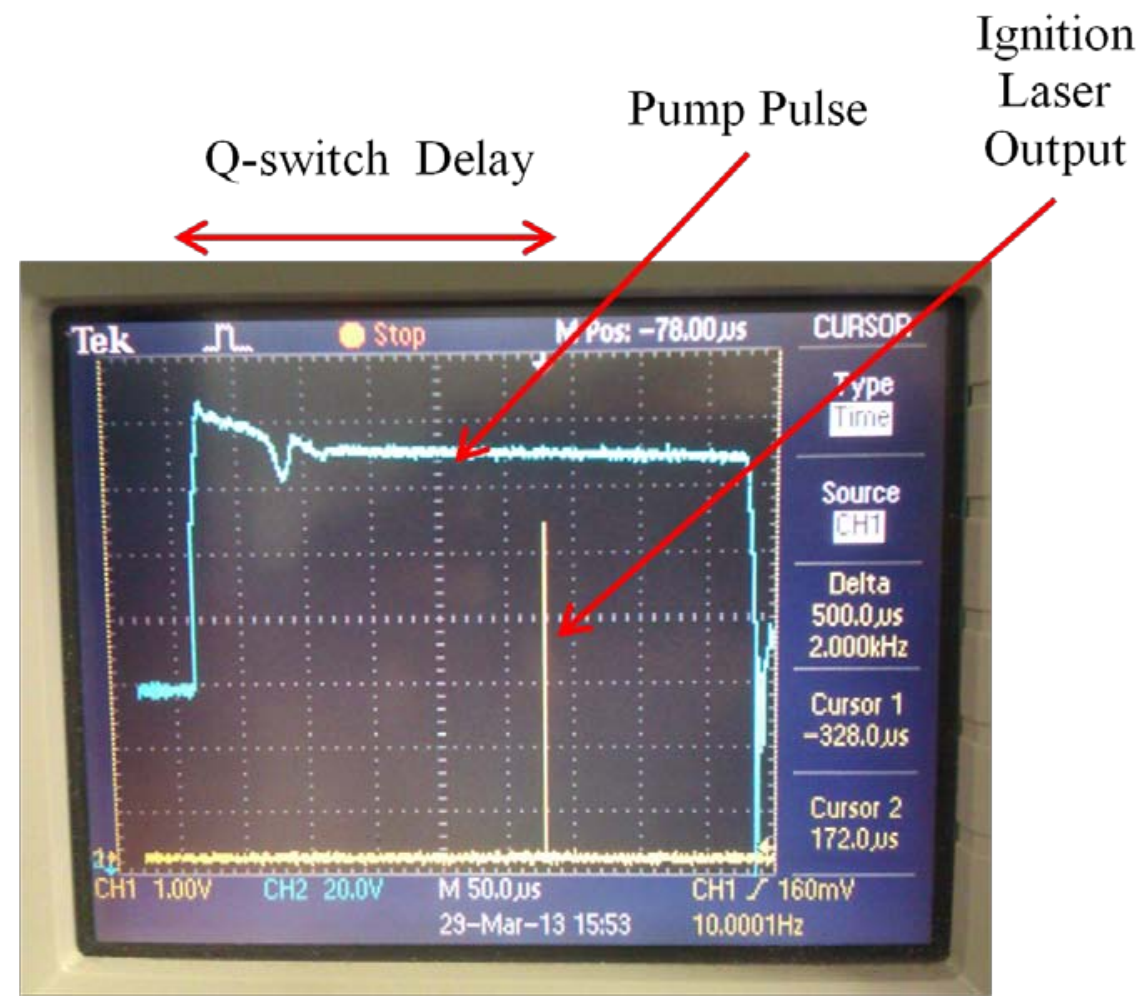

Figure 5: Measurement technique for q-switch delay using an oscilloscope

The technique of q-switching is achieved by inserting a variable attenuator into the resonating cavity of the laser. With the addition of an attenuator in its high state, the losses in the cavity are high (low Q factor) and most of the light which leaves the excitation media does not effectively couple with the cavity and does not contribute to useful output. When the attenuator is switched to its low level, a high Q factor is yielded and the losses in the cavity become low allowing the excited photons to resonate within the optical cavity. At this point, lasing begins.

In a q-switched laser, the gain media is pumped with the q-switch set to high preventing the resulting photons from circulating within the cavity and producing further stimulated emissions within the gain media. Preventing the energy from leaving the cavity causes a large population inversion to occur resulting in a high amount of energy potential within the cavity. After a certain amount of time has passed to accumulate a desired amount of energy, the qswitch changes the quality of the cavity from a high $\mathrm{Q}$ to a low $\mathrm{Q}$. At this point, the buildup of excited photons is able to circulate within the optical resonator and stimulated emission begins. 
Since a large amount of energy has built up over the time, a large amount of energy is released when the $\mathrm{Q}$ of the cavity becomes low. The result is a high intensity pulse of light.

The $\mathrm{Q}$ of the light within a laser cavity is defined as the proportion of the energy that is stored in the cavity to the energy that is lost due to pumping, as shown in Equation 2-4 [27]. This technique of modulating the $\mathrm{Q}$ of the cavity results in a large portion of the stored energy in the laser's cavity to be released in periodic pulsed intervals. This produces a high energy pulse at the lasers output.

$Q=\frac{E_{\text {stored }}}{E_{\text {lost }}}$

The q-switching technique is advantageous for ignition purposes over a continuous wave (CW) lasing system for several fundamental reasons. The peak power output from a pulsed laser system is typically several orders of magnitude larger than the CW output from the same laser system [21]. A comparison of the output from a pulsed laser system and a q-switched laser system is graphically shown below in Figure 6.

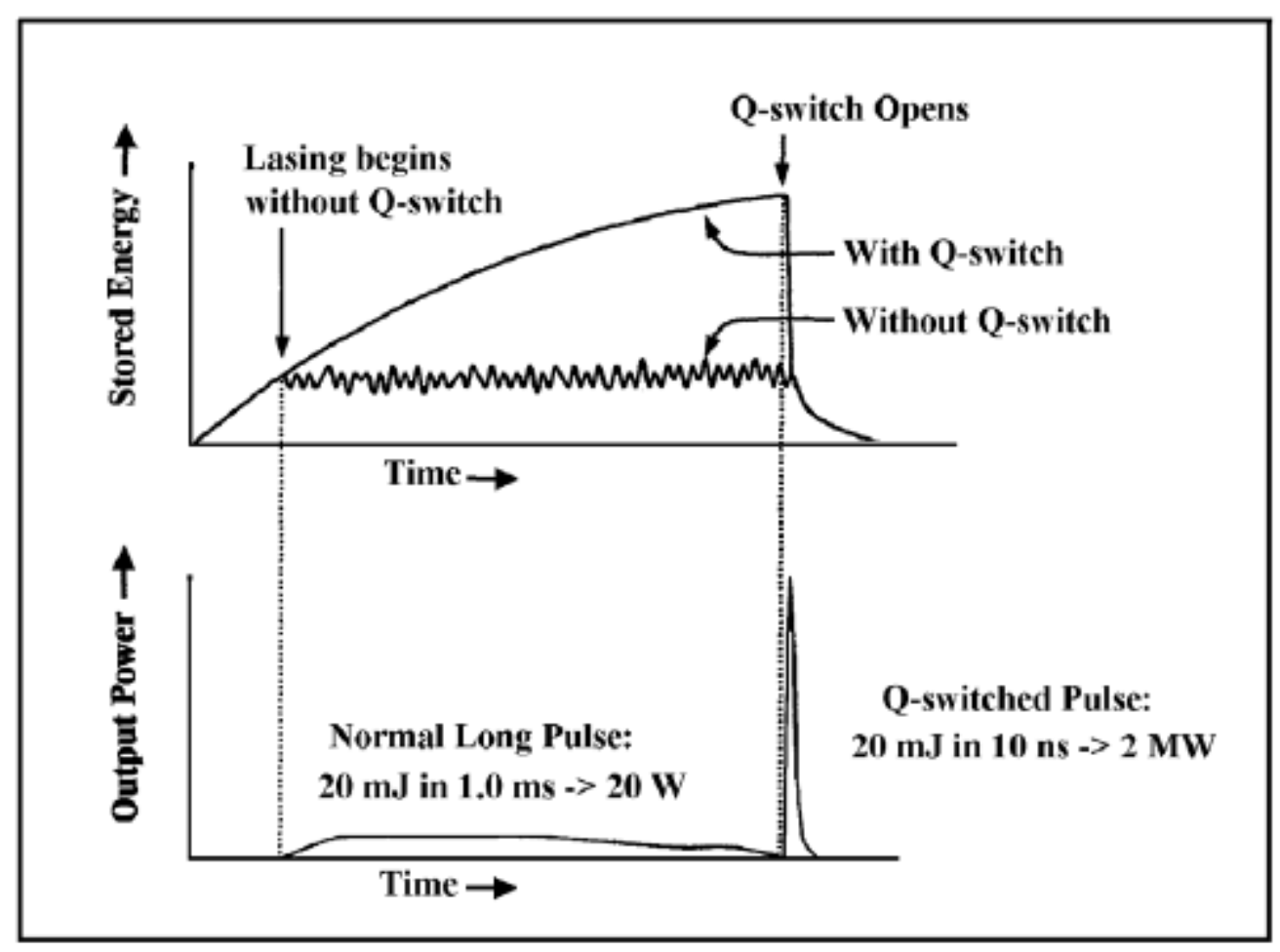

Figure 6: CW and q-switched pulsed laser development [28] 
The upper portion of Figure 6 shows how energy is stored within the laser system as a function of time, while the bottom portion shows the output power of the laser system as a function of time. It can be seen that a q-switched laser system can accumulate and store energy beyond the point which would typically be possible with a CW laser system. Both a q-switched laser and a CW laser will deliver energy to the surroundings, however the difference arises in the time in which the energy is delivered. Typically, there are two types of q-switching techniques, active and passive, which are further explained in the next section.

The time in which energy is delivered from a pulsed laser system is known as the pulse width. The pulse width from a q-switched laser is typically on the order of picosecond or nanosecond scale depending upon the laser's design. For the purposes of this investigation, pulse width is defined as the full width half maximum (FWHM) of the 1064nm optical energy exiting the LSP as a function of time.

\subsubsection{Active Q-Switching}

In an actively q-switched laser, the q-switch itself is a variable attenuator that is controlled through some external means. The trigger mechanism for the q-switch may be actuated through several different means including but not limited to mechanically, electrically or using acousto-optics. With an active q-switch, the recurrence intervals of the output pulse can be precisely controlled to suit the need of the application. An active q-switch not only allows for precision control of the pulse interval, but can be utilized at a very high repetition rate as well.

There are a few reasons why applying an actively q-switched laser towards laser ignition applications is not practical. An active q-switch laser is generally bulky as per its requirements to house an actuation mechanism and accompanying circuitry. Given the complexity of this design, an actively q-switched laser is typically expensive as well. Given these properties, an actively q-switched laser is currently not considered to be practical for mass produced laser spark plugs.

\subsubsection{Passive Q-Switching}

In a passively q-switched laser, the q-switch material acts as a saturable absorber whose opacity has the potential to vary as a function of energy input. It is a material that is introduced 
into the laser's optical cavity whose transmission is dictated by the intracavity photon density. Initially, the losses from the passive q-switch are high and few photons are able to circulate within the cavity. As pump energy continues to be applied to the excitation media, the population inversion continues to build and produce low level stimulated emission with a steadily increasing degree of photon circulation until the saturation threshold of the absorber is reached. When the light intensity exceeds the threshold of the q-switch within the laser cavity, the cavity losses are reduced and the transmission of the material rapidly increases allowing the stored energy to be released into the cavity. This transition to an increased transmission is known as bleaching. Unlike an active q-switch where an external means of modulation is required for operation, a passive q-switch's operation is a strict function of the material's absorption proprieties and intracavity photon density. The most typical means of controlling this type of qswitch is by control of the pumping frequency. Output from a passively q-switched laser can be controlled via the pump pulse duration and pump power applied to the gain media. The combination of the passively q-switched laser's initial transmission, OC and neodymium concentration influence the output characteristics as well. In this technique, maximum excited state production is a function of the time it takes the saturable absorber to reach its transition threshold.

Passively q-switched lasers are considered to be practical for mass production of laser spark plugs. Although indirect control over the $\mathrm{Q}$ of the cavity presents a certain amount of error that may lead to cycle-to-cycle variation of the resulting output pulse, they are far less expensive than active q-switches, more easily mass produced and can be manufactured much smaller as well. A passive q-switch is an ion doped material which belongs to the transition material family. The q-switch material chosen for a particular laser setup should operate in conjunction with a given gain medium based on the stimulated emission from the gain media [21, 29]. In the case of this experimentation, a Cr:YAG q-switch was used in conjunction with a Nd:YAG gain medium.

Under operation, a lasing medium undergoes a significant thermal variation from the addition of the pump energy. Since the excitation media of a passively q-switched laser is best controlled through modulating the pump source, the media undergoes a thermal cycling process which has been found to affect the average power output of the laser [30]. Transient thermal 
effects have been found to strongly effect the $1064 \mathrm{~nm}$ output from a passively q-switched Nd:YAG laser rod [30]. Attempts have been made to control the naturally occurring temperature fluctuations of an active medium by use of techniques such as heat capacity mode (HC) of operation and continuous cooling (CC) with success. The heat capacity mode of operation is defined as where no cooling takes place during lasing and the waste heat from the excitation process is stored within the gain medium. Continuous cooling is a process in which the excitation media is cooled in periodic intervals between the lasing cycles. The rate of cooling is limited by the fracture stress of the gain medium and has been shown to result in an increase in power output and provides for a reduction in optical distortions [31].

As shown in Figure 7, the average power output increases with the increased addition of pumping input power from a solid-state Nd:YAG laser [30,32]. Under these modes of operation, the thermal gradients were minimized through their control techniques resulting in significantly reduced thermo-optic distortions and improved $\mathrm{M}^{2}$. Also shown in Figure 7, a higher average power output was achieved under $\mathrm{CC}$ as opposed to all other $\mathrm{HC}$ modes of laser operation. Adequately cooling gain media in a passively q-switched laser gives a level of control over the output energy [30].

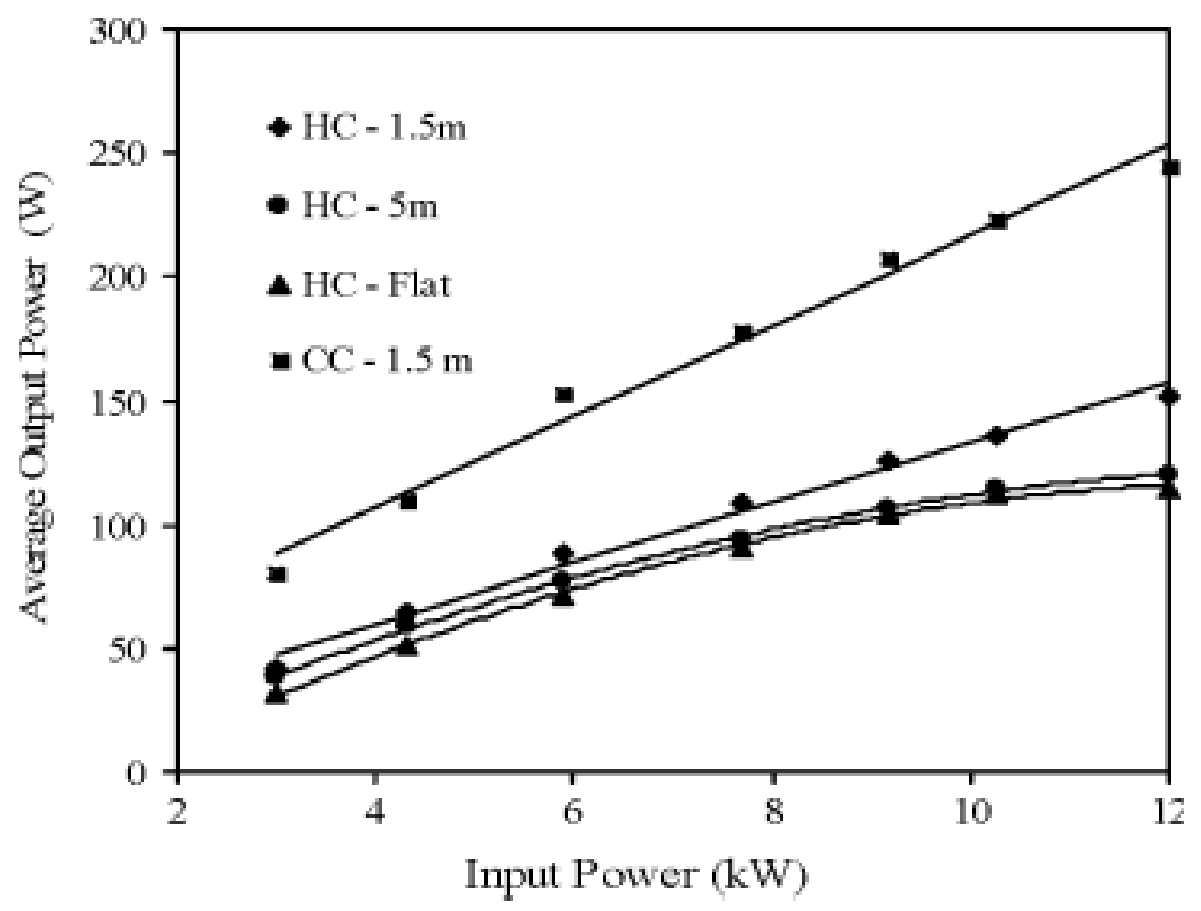

Figure 7: Output power of an Nd:YAG laser as a function of input power in $\mathrm{HC}$ mode and its comparison with CC mode [30] 
The Cr:YAG crystal has unique properties allowing it to be efficiently used in conjunction with Nd:YAG as an active laser medium. The absorption properties of a Cr:YAG qswitch compliment the stimulated emission from an $\mathrm{Nd}: \mathrm{YAG}$ gain media. The initial transmission of the q-switch is engineered to fit the application where the initial transmission is dependent upon the doped chromium concentration and the optical path length [21]. The temperature dependence upon the operation of the Cr:YAG q-switch is of importance when being applied towards LI applications. With an increase in temperature, the initial transmission of the Cr:YAG crystal has been found to increase as shown in Figure 8 [33].

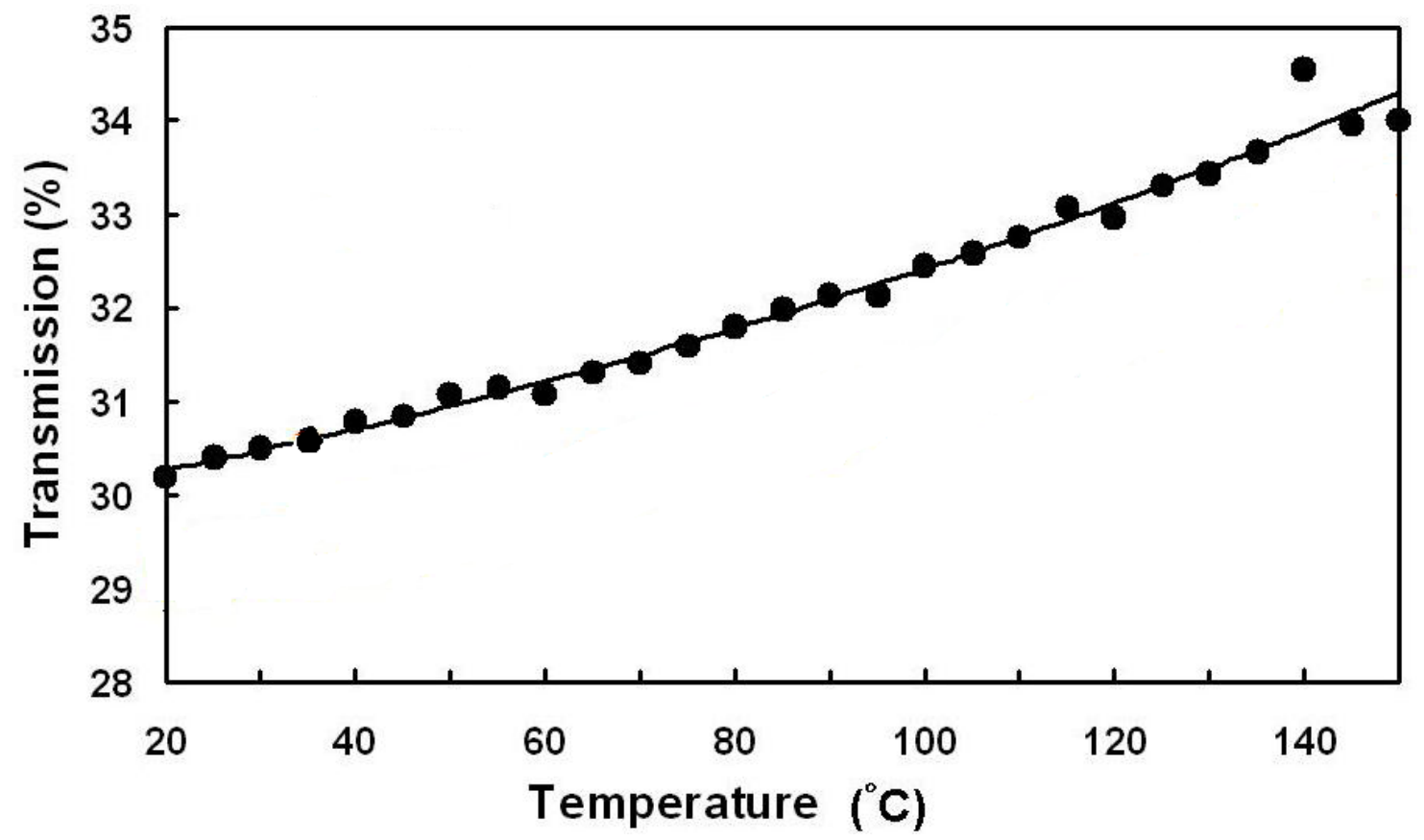

Figure 8: Initial transmission of a Cr:YAG crystal as a function of crystal temperature [33]

As shown by McIntyre and Woodruff in Figure 9, q-switch delay decreases with increasing initial transmission for a given OC and pumping frequency. This work shows that there is a relationship between initial transmission and q-switch delay. Upon analysis of Figure 8 concerning the operational thermal setpoints applied in this investigation, it is shown that the initial transmission of the q-switch may have changed by as much as $1 \%$ across the range of temperatures tested. The change in the initial transmission of the q-switch may alter the q-switch delay, however, given the small amount of change, research performed by Bass et al. suggests a negligible effect on the delay [34]. 


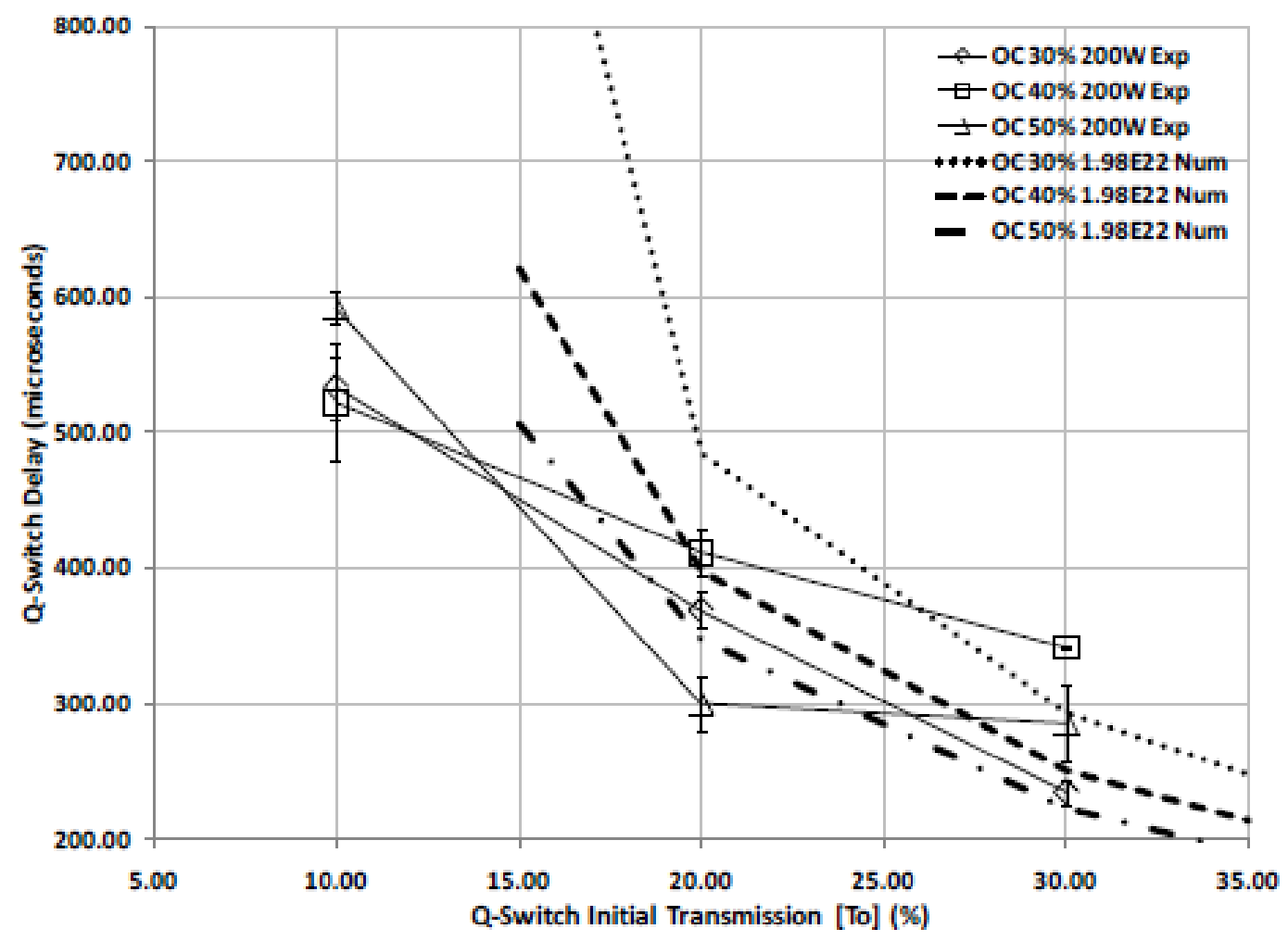

Figure 9: Q-switch delay data for an end pumped laser plotted with numerical data as a function of initial transmission [29]

Initial studies to determine the thermal influence on the operation of a laser with a Cr:YAG saturable absorber have found that the output energy can be maintained over a wide temperature range. Tsunekane and Taira have tested and reported on this for a wide range of temperatures [33]. It was found that output energy increases with increasing temperature as shown in Figure 10. This is due to an increased saturation absorption coefficient with respect to increasing temperature. As temperature increases, the molecular absorption cross section of the gain media increases in size. The absorption cross section is a measure of an atoms interaction cross section to absorb a photon at a certain wavelength. As cross section increases, there is a higher probability that a photon come in contact with the atom. This increases the absorbed photon percentage allowing for a greater number of electrons to enter an excited state, resulting in higher output energy at elevated temperatures. 


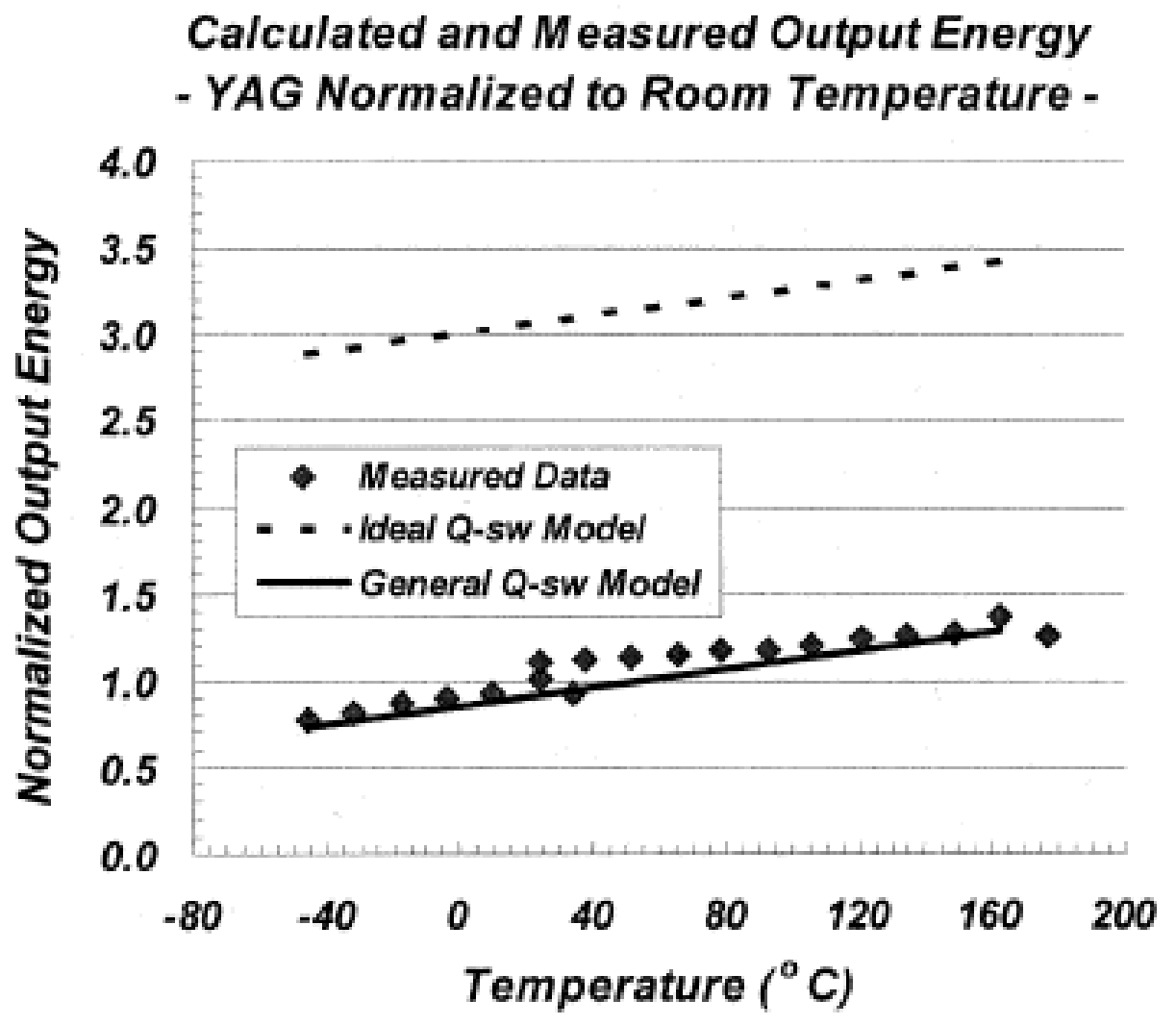

Figure 10: Calculated and measured output energy of an end pumped Nd:YAG laser [33]

Research has shown that the thin film coatings and intra cavity optics do not show any temperature dependence in this range, and that the absorption cross sections of the Cr:YAG qswitch remain virtually independent of temperature as well [34]. The energy output from a passively q-switch laser is found to be a strong function of the temperature of the gain medium. Equation 2-7 and Equation 2-8 describe the linear relationship between output energy and temperature in an Nd:YAG solid-state laser assuming all other parameters are held constant where $E$ is the output energy, $T$ is the temperature and $b_{0}$ is output energy at an initial temperature.

$E(T)=T \frac{d E}{d T}+b_{0}$

$\frac{d E}{d T}>0$ 
As indicated by Equation 2-8, the slope of the line in Equation 2-7 is always greater than zero. This is confirmed by the work of Shah et al in Figure 7 [30], and Tsunekane and Taira in Figure 10 [33]. The increasing slope of the line is determined by not only the operating temperature of the laser, but is a function of the temperature variance due to thermal cycling from absorbtion of the pump energy as well.

\subsection{High Peak Power Delivery through Fiber Optic Cables}

Fiber optic cables are useful for spatially complex energy transfer. Fiber cables are attractive due to their flexibility, which is needed for routing energy from a remotely located laser and multiplexer to a designated engine cylinder. A durable exterior jacket on the fiber is also desired to absorb vibrations from the engine and to protect the core-cladding interface. The amount of induced stress residing in the core and cladding of the fiber optic cable has been found to have a significant impact on the beam's intensity profile at the fiber's output. A fiber in a relaxed state where the influence of external stresses is minimized provides a more desirable $\mathrm{M}^{2}$ value than a fiber influenced by a force such as bending or surface loading. An exception to this is a uniformly distributed bend or coil, which will cause a reduction in transmission efficiency, but will also cause the fiber to lose its higher order modes resulting in better $\mathrm{M}^{2}$ [22].

The intensity profile of the fiber's output is a critical aspect when attempting to generate a laser spark using high peak power fiber delivery. The preparation of the input and output ends of the fiber cable is critical to obtain the best possible laser pulse transmission and output beam quality. A uniformly spatial beam distribution across the fiber face is strongly desired and is obtained by having a perfectly flat surface polish. The polishing process is a laborious procedure which must be conducted with the utmost cleanliness and care to ensure the final quality of the fiber's face. A poor surface finish will lead to unwanted light scattering, Fresnel back reflection, a high $\mathrm{M}^{2}$ value and may cause damage to the fiber via mode coupling and local intensity peaks [35].

There are several styles of fiber optic cable which vary geometrically and in material composition to compliment a specific application. Only a select few have the necessary features to accommodate the requirements for laser ignition. These fibers must be able to transfer megawatt level power laser pulses of adequate beam quality to allow for the breakdown threshold of a gas to be reached at the fiber's focused output. For gases such as methane, the 
breakdown intensity when stoichiometrically mixed with air is approximately100 $\mathrm{GW} / \mathrm{cm}^{2}$ which presents an optically challenging situation for pulsed laser delivery [8, 36, 37]. Optical breakdown through fiber optic cables has been achieved with energies as low as $4 \mathrm{~mJ}$ at $10 \mathrm{~ns}$ pulse durations, however ignition of most $\mathrm{A} / \mathrm{F}$ mixtures is achieved with pulse energies of $\sim 15$ $\mathrm{mJ}$ at $15 \mathrm{~ns}$ pulse durations [38, 39].

\subsubsection{Multimode Solid-Core Step-Index Fibers}

Multimode solid-core step-index fibers are considered to be the most ideal fibers for high intensity pulse transmission. Their construction is of a constant refractive index throughout the core of the fiber followed by an abrupt index increase at the cladding interface. This allows for the majority of the transmitted pulse to reside in the fiber's core. A coating and jacket layer surround the cladding provide the fiber with more resistance to external influences such as stress, bending, abrasion and heat. The core/cladding ratio is available in several different configurations dependent upon wavelength and application, typically with a core size between 50-1000 $\mu \mathrm{m}$. Under ideal fiber operation, total internal reflection will occur at the core cladding interface for all of the operating modes that the fiber supports. The consistency of a solid core's refractive index results in a more uniform intensity profile at the fiber's output. It is this quality that makes step index fibers attractive for plasma spark generation and laser ignition applications [22].

Recent advances in the research of step index fibers (core/clad=400/720) have shown that the transmission of $3 \mathrm{~mJ}$ at $10 \mathrm{~ns}$ pulse durations using $1064 \mathrm{~nm}$ light is capable of producing $100 \%$ plasma spark generation in air at atmospheric pressure [39]. Although this energy is below the minimum energy required for ignition of applicable gases, much higher transmission energies can be achieved at longer laser pulse durations. This presently makes the solid-core step-index fiber optic cable the most applicable style of fiber for pulsed transmission and laser ignition applications [22]. This style and size of fiber is small enough to still maintain an appropriate $\mathrm{M}^{2}$ and large enough to be structurally resistant to induced perturbations.

Vibration can lead to unwanted mode coupling when using fiber optic cables. A laser beam passing through an optical fiber may have several propagation modes even when the fiber is kept straight and in a relaxed state. Vibration inherently yields oscillatory bending which induces stress within the fiber. This causes a non-linear interaction between the propagation 
modes leading to high local intensities within the fiber. Under low energy applications this is not of major concern, however when transmitting energies close to the breakdown threshold of the fiber's fused silica core, damage to the fiber may occur. A fiber that has reached its breakdown threshold in a localized volume is shown in Figure 11.

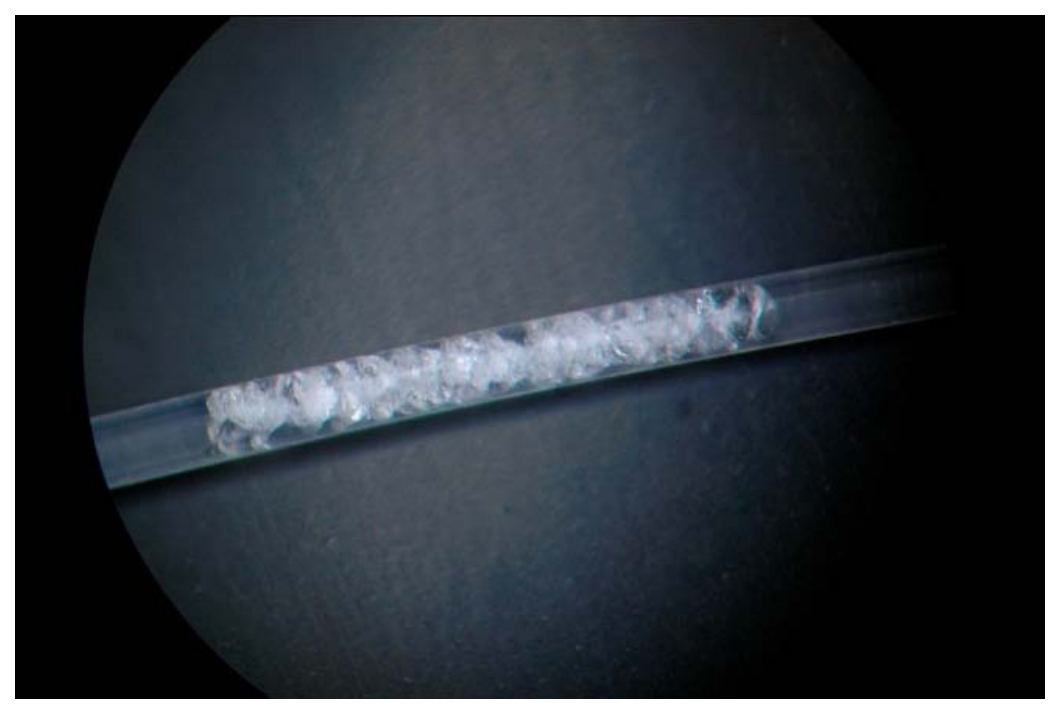

Figure 11: Solid core step index fiber with exceeded damage threshold of $\sim 1-5 \mathrm{GW} / \mathrm{cm}^{2}$ [22]

In vibration induced stress situations where the breakdown of the fiber's core is not reached, adverse effects from mode coupling can occur at the fiber's output as shown in Figure 12. Mode coupling can occur via several different mechanisms in the fibers core. These mechanisms are primarily due to changes in geometry that affect the internal reflection angle of the fiber. This can make it extremely difficult to produce a plasma spark given the distorted intensity profile at the output. In an ideal case, a plasma spark is most easily achieved with a Gaussian profile beam. Vibration leads to significant nonuniformity of a laser's output intensity profile and is therefore a major concern when applying this technology to the oscillation intensive environment of an IC engine. 

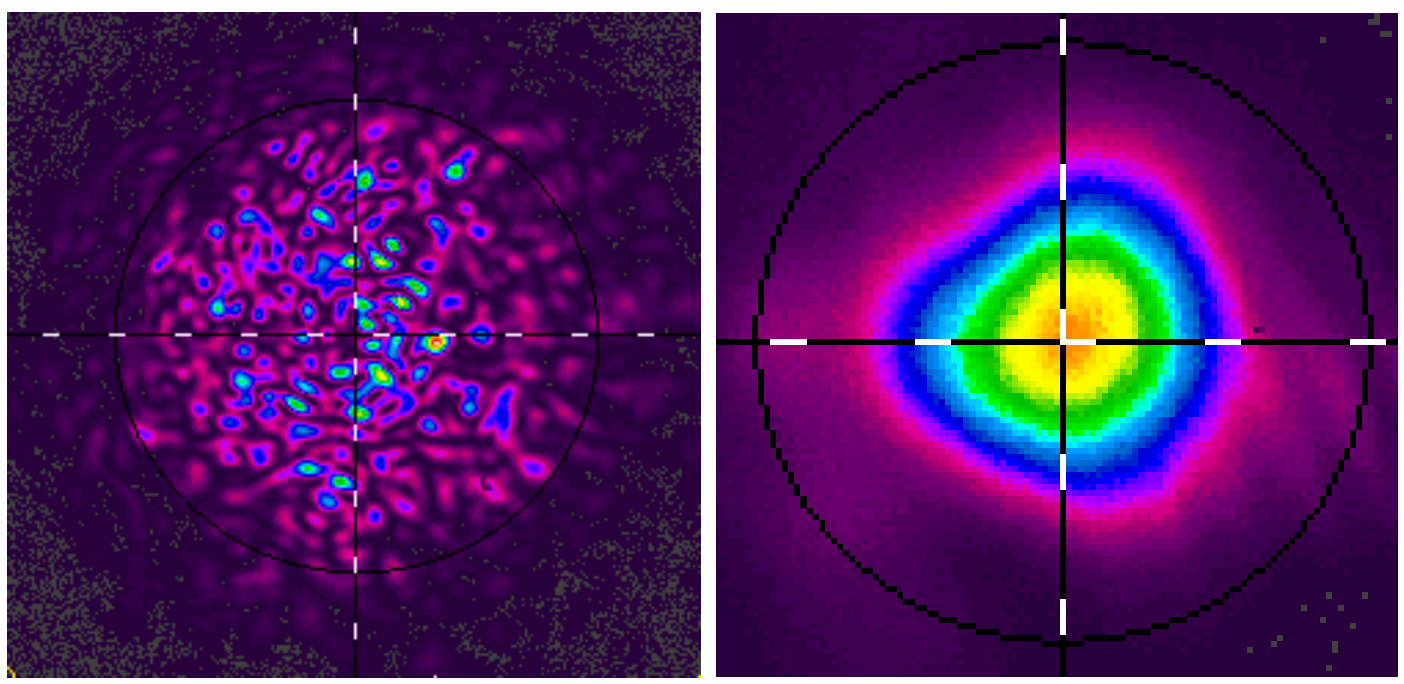

Figure 12: Output beam intensity profile due to mode coupling from externally induced stress (left), and desired beam quality output (right) [22]

\subsection{Current Laser Ignition Technology}

To date, the majority of LI research has focused on stationary large bore natural gas engines. Traditionally, a laser based ignition system is very sensitive to the heat and vibration produced by the operation of an IC engine, requiring a large portion of the experimental apparatus to be remotely located $[4,5,10]$. Also, the cost of a laser system capable of producing the output characteristics necessary to achieve optical breakdown is relatively expensive and consequently, a multiplexed strategy is often adopted for multiple cylinder engines $[4,5,6,8$, 10]. For these reasons, stationary engines have been highly targeted for the application of this technology because of the ability to remotely locate a desired component of the ignition system. Figure 13 shows a strategy for multiplexing the low peak pumping energy through fiber optic cables to the respective laser plug mounted directly on the cylinder. 


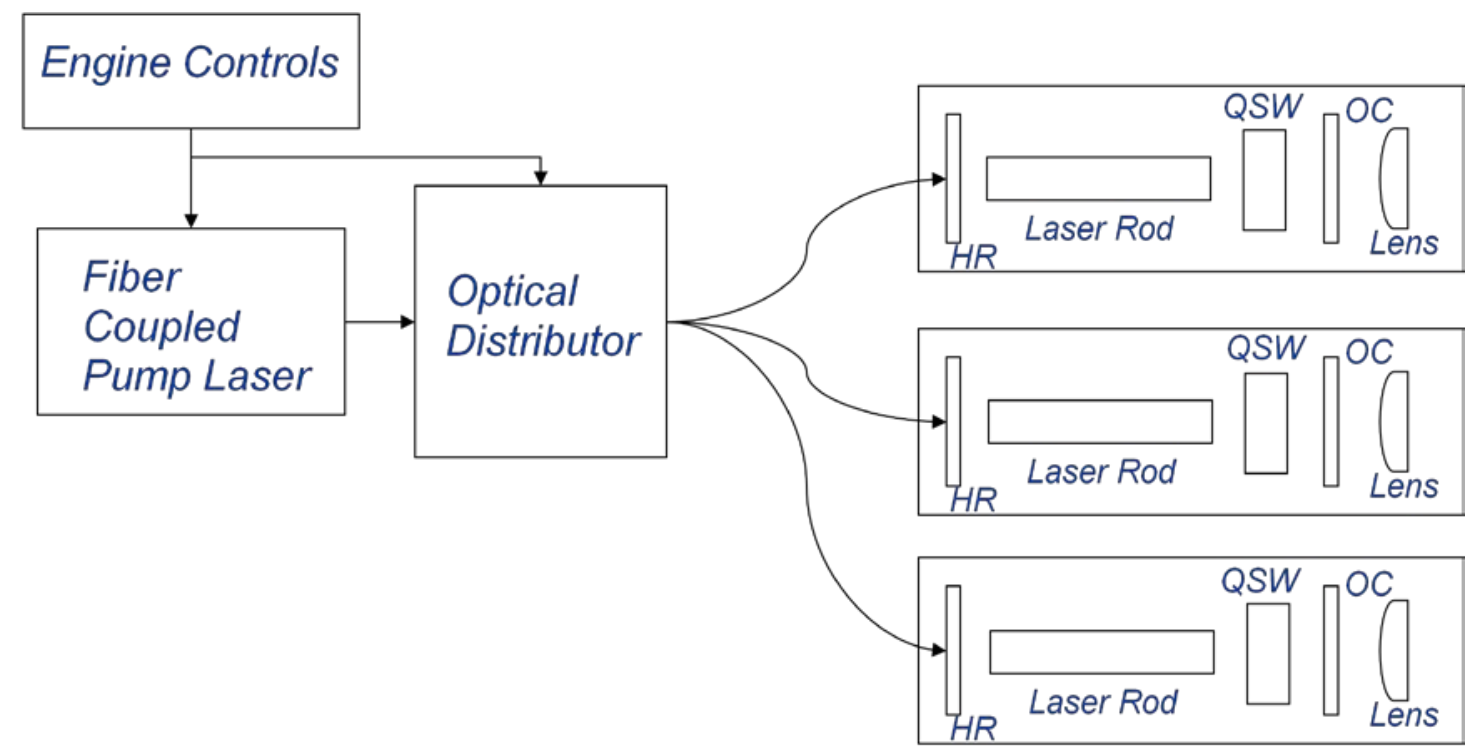

US Patent 7,421,166

Figure 13: Strategy for multiplexing the pump energy to each respective ignition laser [21]

The problem with utilizing a laser as an ignition source fundamentally resides in its ability to be mass produced cost effectively, and to be rugged enough to operate in a harsh engine environment. A laser of this magnitude can be a sensitive and costly instrument making it a challenge to implement this technology for ignition applications even in a controlled laboratory environment. Design of this ignition system must be focused towards durability, reliability and cost effectiveness for it to be accepted for industrial applications and long term usage.

\subsubsection{Laser Spark Plug Approach}

Current research conducted by NETL has allowed for the creation of a Nd:YAG, solidstate laser operated at $1064 \mathrm{~nm}$ that can be directly positioned at the spark plug port of a cylinder head [40]. This design is far more impervious to temperature and vibration than previous LI strategies such as open beam transfer or high power transmission through fiber optic cables, since open beam transfer requires the entire apparatus to be remotely located due engine perturbations and fiber optic cables are extremely susceptible to vibration. The number of components involved in the construction of the LSP was minimized as to allow for a significant reduction in cost for mass production. In this design, the passively q-switched ignition laser is directly mounted on the cylinder head of the engine and is optically pumped via a pulsed 200 
watt fiber-coupled diode laser bank operating at $808 \mathrm{~nm}$ [40]. A photograph of the prototype laser spark plug mounted on a research engine is shown in Figure 14. The pumping energy from the diode laser is transmitted to the ignition laser via a multi-mode step-index solid core fiber optic cable with a $400 \mu \mathrm{m} / 720 \mu \mathrm{m}$ core/cladding ratio. This pump energy is significantly less than that of the breakdown threshold of the fiber's fused silica core and does not damage the fiber. Although vibrational effects can still lead to mode coupling in the fiber and an inconsistent output intensity profile, this is not of major concern as the only purpose of the energy transferring through the fiber is to pump the ignition laser. The pumping of a laser's media does not require a precisely uniform intensity profile to be effective.

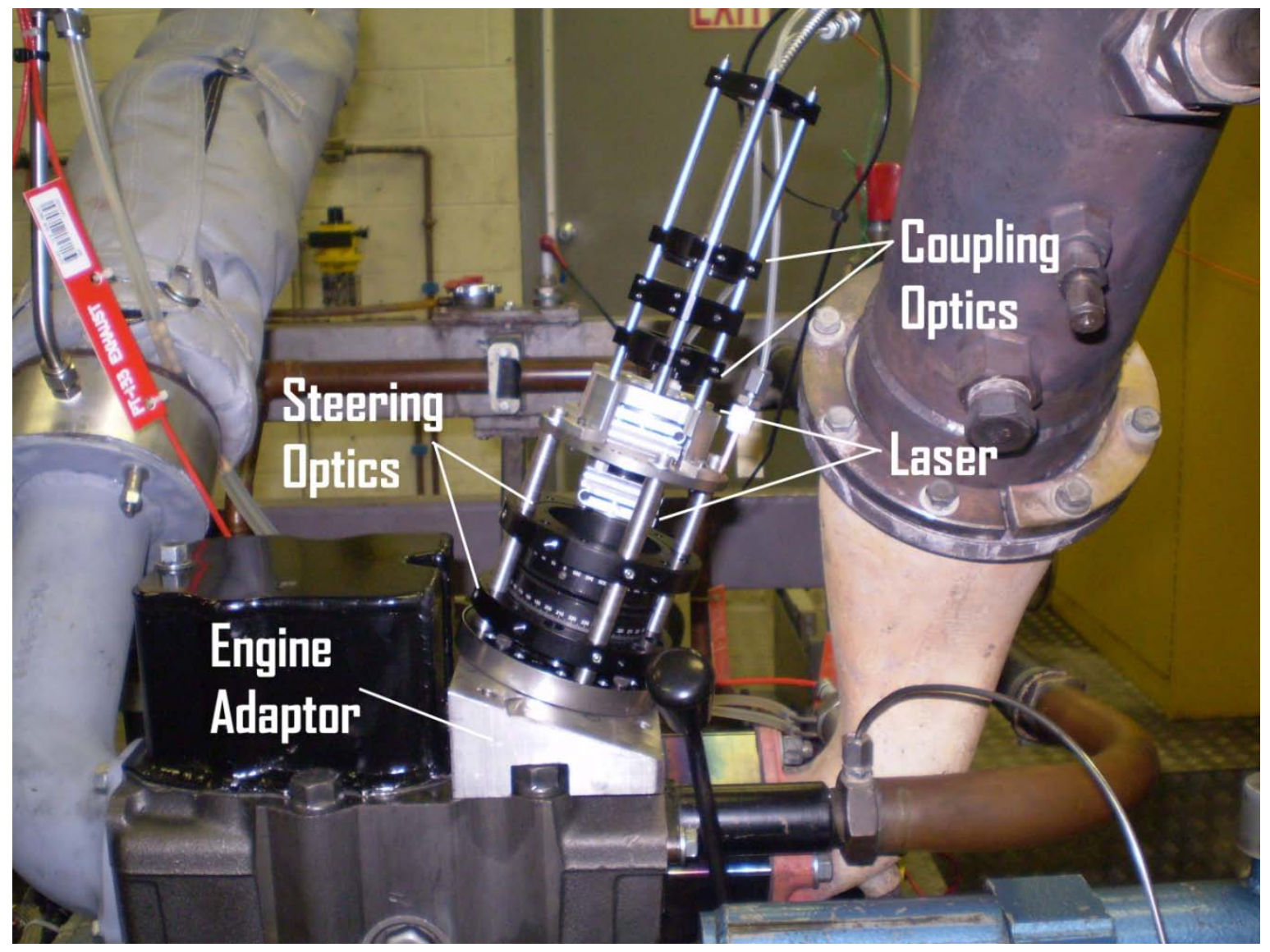

Figure 14: The NETL prototype laser spark plug was initially mounted onto a research engine fueled by NG or a NG/hydrogen mixture [40]

The LSP was originally installed and operated on an engine located in a test cell at the U.S. DOE's NETL facility in Morgantown WV. The test cell facility consisted of a Ricardo Proteus single cylinder engine and dynamometer in addition to the accompanying control and 
instrumentation necessary for modifying the operational parameters of the engine. The LSP successfully ignited the $\mathrm{A} / \mathrm{F}$ mixture in the engine at several different setpoints which have been fully defined in the literature $[29,40]$. 


\section{EXPERIMENTAL SETUP}

The LSP was mounted on a vibration exciter system and wrapped with heat tape. The LSP was mounted in three different orientations. In each of the three orientations, matrices of thermal, oscillatory and acceleration setpoints were employed. The following sections describe the experimental setup, data collection procedure and the variables of concern in detail.

\subsection{Vibration}

In order for a LI system to be considered for igniting an $\mathrm{A} / \mathrm{F}$ mixture in an IC engine, the effects that induced external perturbations from the engine have on laser operation must be quantified. The precise frequency and amplitude at which an engine vibrates is somewhat complex to characterize as several conditions such as location, plug angle, engine speed/load and the engine's design must be taken into consideration. To obtain some preliminary information about engine vibration, two engines have been outfitted with an accelerometer and a thermocouple to record the engine's vibrational frequencies, accelerations and temperatures at a given setpoint. Both of these engines were mounted inside the engine compartment of vehicles, and measurements were taken with the vehicles stationary and on flat ground. The acceleration data collected is graphically shown in Figure 15.
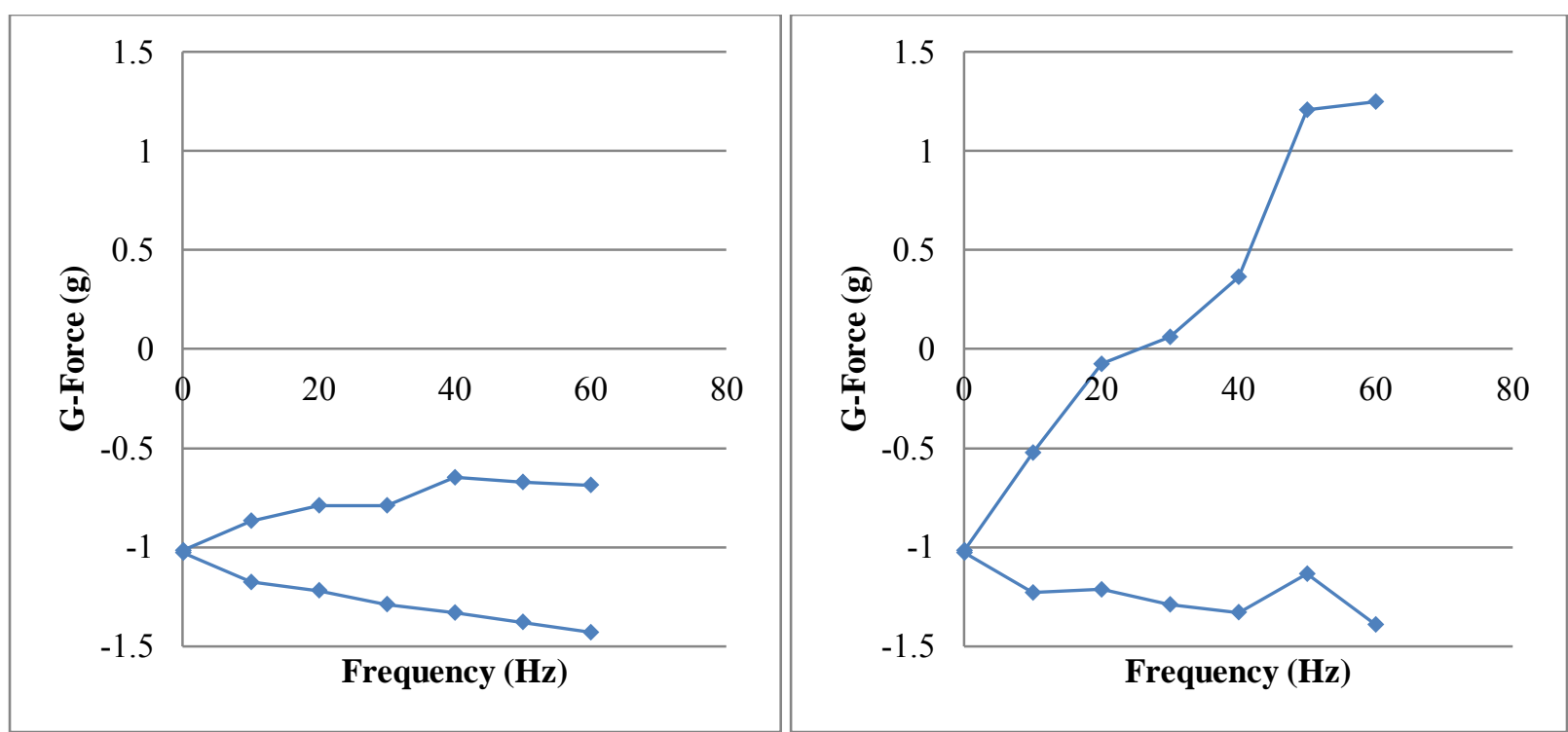

Figure 15: G-force as a function of frequency in the $\mathrm{x}$ axis for a 3.4L V6 5VZ-FE gasoline engine (left) and for a 7.3L V8 Powerstroke turbo diesel (right) 
The accelerometer data reveals that oscillations occur simultaneously in all three Cartesian directions, however, only the primary "x axis" is taken into consideration here due to the significantly smaller magnitudes of the other two axes. Attempts were made to mimic the acceleration magnitudes for a given frequency setpoint, however, due to limitations set forth by the vibrational testing equipment this was not possible.

\subsubsection{Vibrational Testing Equipment}

\subsubsection{Shaker}

The vibration system used for this work was a single axis B\&K Vibration Exciter Type 4808 Shaker shown in Figure 16, which was used in conjunction with at B\&K Power Amplifier Type 2719 [41]. Specification sheets for each of these pieces of equipment may be found in Appendix-B. This series of permanent magnet shakers has a wide frequency response band and are comprised of electro-dynamic transducers that are capable of producing linear sine wave motion with a maximum orthogonal vector force of $25 \mathrm{lbf}$ without assisted air cooling. In order to allow for maximum performance to be achieved, OEM provisions have been made to the vibrator body to accommodate a forced air cooling system from an auxiliary cooling fan, resulting in forces of approximately $42 \mathrm{lbf}$ to be achieved.

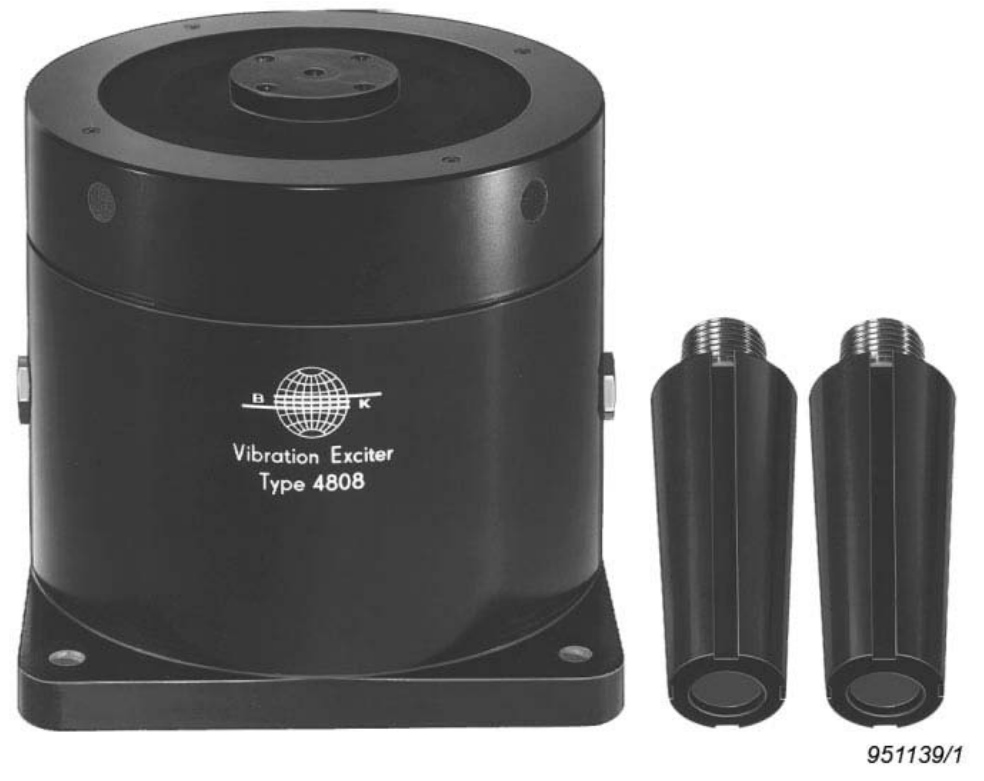

Figure 16: Single Axis B\&K Vibration Exciter Type 4808 Shaker [41] 
The shaker is capable of oscillating in a large useable frequency band of $5-10,000 \mathrm{~Hz}$, however the operational frequency of an IC engine are significantly less than this. The rated speed of a NG engine varies not only between manufacture, but varies amongst applications as well. For example, the rated speed of a natural gas engine in most transit busses range from 1,600-3,500 RPM, while the rated speed of stationary large bore natural gas engines used for the transfer of NG in a pipeline range from 600-1,800 RPM. Since the purpose of this investigation was to provide a stepping stone for the advancement of laser ignition for multiple applications, a range of frequencies up to $60 \mathrm{~Hz}$ was analyzed that simulates a wide range of $\mathrm{NG}$ engine operational frequencies up to 3,600 RPM.

Adapters were constructed in order to connect the laser to the vibration exciter. The LSP was then mounted on the vibration exciter in three different orientations of $0^{\circ}, 45^{\circ}$ and $90^{\circ}$ relative to the horizon. A picture of the LSP in the $90^{\circ}$ orientation mounted on the exciter is shown in Figure 17. 


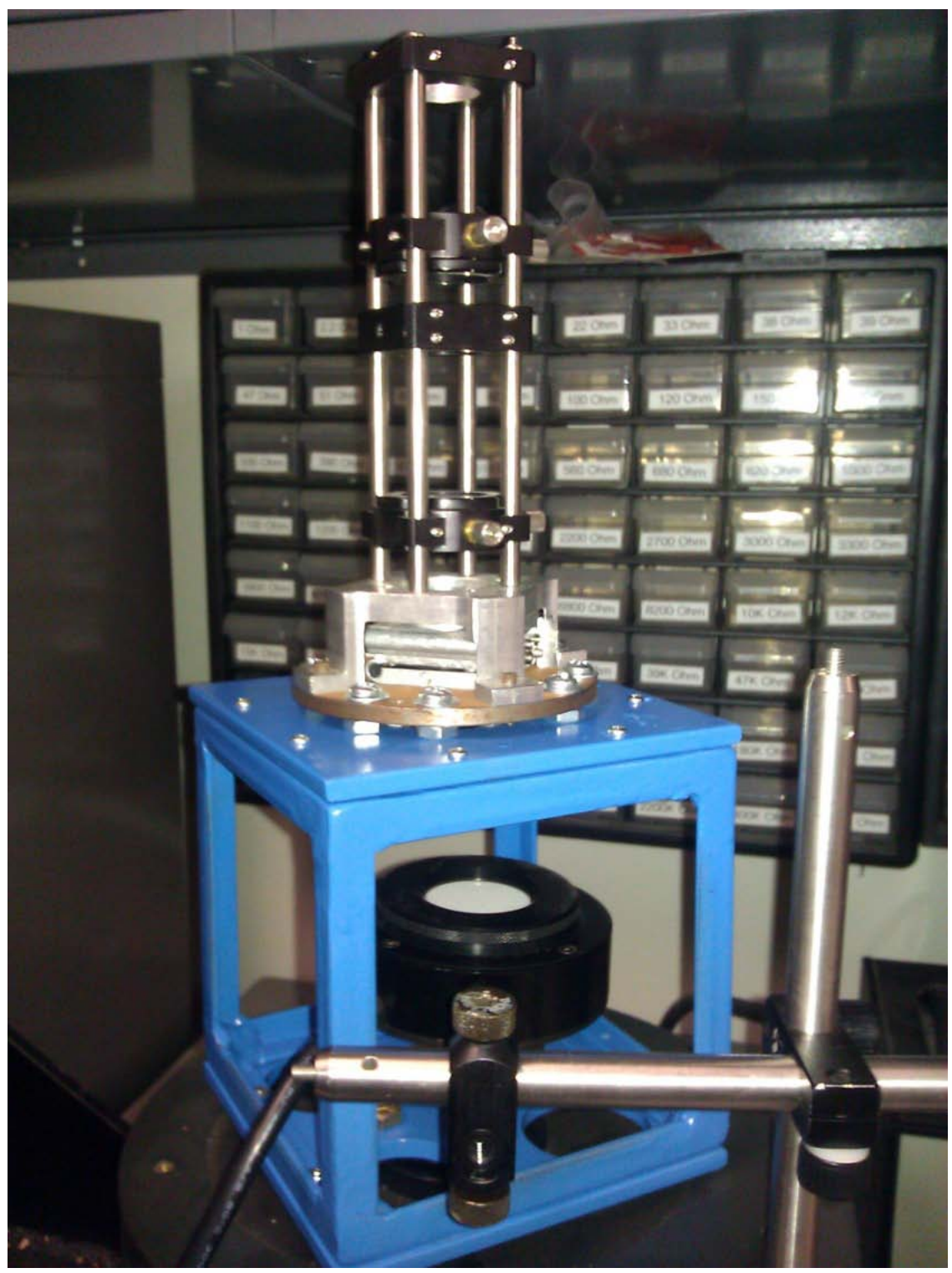

Figure 17: Laser spark plug mounted on the vibration exciter with a $90^{\circ}$ orientation.

\subsubsection{Accelerometer}

To verify proper magnitude of oscillation and to provide a means of positive feedback for the acceleration setpoint, an accelerometer was mounted on the laser adapter. The accelerometer used for the oscillation verification was a UM6 Ultra Miniature Orientation Sensor. It is a triaxial accelerometer with a built in rotational gyroscope capable of measuring $\pm 2 \mathrm{~g}$ up to $500 \mathrm{~Hz}$ [42]. 


\subsection{Temperature}

To measure the expected temperatures that the LSP was exposed to, a thermocouple was mounted on each of the engines used to collect the preliminary acceleration data. Since temperature varies with the speed and load of the engine, multiple engine speed setpoints were used to collect this data in accordance with the vibrational test matrix discussed in Section 4.1.4. This provided a means of quantifying the expected temperatures that the LSP will be exposed to, and directly translated to the thermal setpoints in the test matrix. The thermocouples were located as close as possible to the top of the combustion chamber for each engine without physically coming in contact with any portion of the engine as to measure the local air temperature. In this LI strategy, the LSP is mounted in such a manner that conduction heat transfer to the LSP is minimal and the primary means of heat transfer can be assumed to be convective and radiative. For the $3.4 \mathrm{~L}$ and $7.3 \mathrm{~L}$ engines, the recorded operating temperatures were approximately $130^{\circ} \mathrm{F}$ and $140^{\circ} \mathrm{F}$ respectively.

\subsubsection{Temperature Testing Equipment}

\subsubsection{Heat Tape and Insulation}

The LSP was wrapped with Omega: Medium Temperature Heat Tape SRT051-080 and heated to temperatures as high as $140^{\circ} \mathrm{F}$ to simulate the environment in which it will be operating on an engine. The heat tape was six feet in length and one inch wide with a maximum heat range of $212^{\circ} \mathrm{F}$. This particular coil type heat tape was chosen due to its inherent versatility to adapt to complex geometries. A picture of the LSP wrapped in the heat tape is shown in Figure 18. 


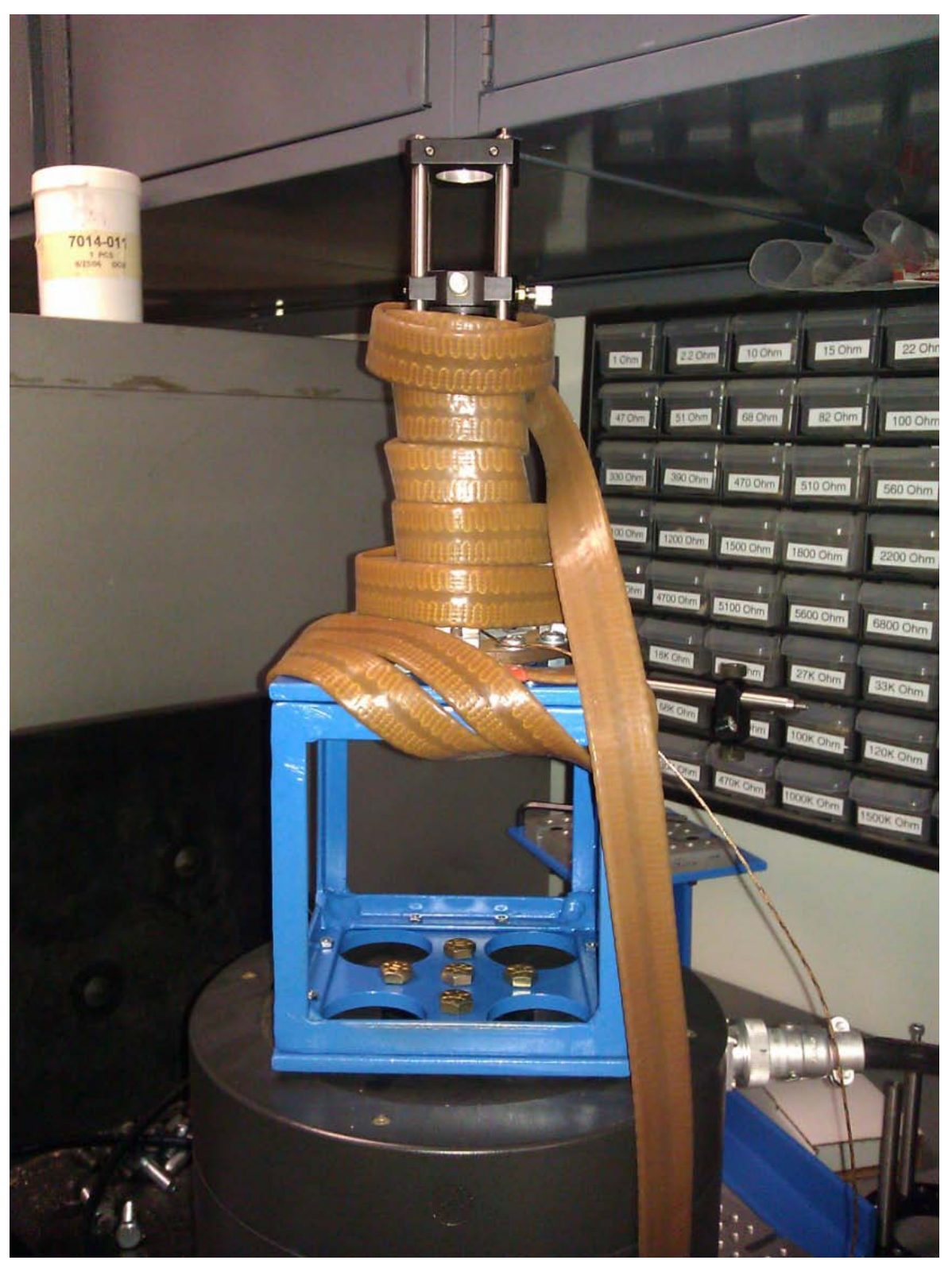

Figure 18: The laser spark plug mounted in a $90^{\circ}$ orientation wrapped with the heat tape.

Once the entire LSP was wrapped with the heat tape, its temperature was controlled with a proportional integral derivative (PID) controller to minimize transient effects from repeated heating and cooling of the LSP as to minimize overshoot and steady-state error. The LSP was heated to the specified setpoint in the test matrix via a temperature controller using a thermocouple for feedback. 


\subsubsection{Temperature Controller}

An Omega PID based Temperature Controller- CNi3243-C24 was able to control the temperature of the LSP at each thermal setpoint in the test matrix with an accuracy of $\pm 0.9^{\circ} \mathrm{F}$. The control thermocouple was mounted directly to the housing of the LSP so it was in contact with the LSP and the heat wrap. The feedback signal from the control thermocouple was used for the thermal setpoint of the heat tape. Due to the location of the thermocouple mounting on the LSP, the heat wrap and the exterior of the LSP became much hotter than the interior when the setpoint temperature was initially set. For this reason, a substantial amount of time was allowed for the temperature to stabilize throughout the LSP. Temperature was monitored in several locations within the LSP assembly as well to monitor the difference in temperature across the LSP as the testing was conducted, but also to act as a failsafe mechanism if the control circuit were to malfunction.

\subsubsection{Thermocouples}

The LSP was outfitted with Omega Cement-On Style J-Type thermocouples in order to supply a feedback signal to the Omega PID based Temperature Controller- CNi3243-C24 and data acquisition (DAQ) system. The small size of these thermocouples allowed for local pinpoint temperature measurements to be made across the LSP. Thermocouples were mounted in various locations on the LSP including the OC housing, high reflector housing, and the subminiature version-A (SMA) fiber connector for monitoring, and on the heat wrap for control.

In order to ensure an accurate temperature determination for each of the subject components of the LSP, thermocouples and accompanying DAQ system components were calibrated in accordance to 40 CFR 1065.315 with a National Institute of Standards and Technology (NIST) traceable thermocouple simulator [43]. The thermocouple calibrator was a certified NIST traceable Fluke 714 thermocouple calibrator. The thermoelectric electromotive force (EMF) values of the J-type thermocouples were calibrated in 10 equally spaced intervals across a temperature range of $60-160^{\circ} \mathrm{F}$ to encompass the temperature range being measured on all the LSP components. Simulated temperature as a function of thermocouple temperature was plotted and upon linear regression analysis the $\mathrm{R}^{2}$ curve fit was found to be greater than 0.998 in 
each case per NIST requirements [44]. The specification sheet for the thermocouples can be found in Appendix-B.

\subsection{DAQ System}

Data acquisition and control was conducted using National Instruments (NI) devices in conjunction with a desktop computer. A NI DAQ Card (PCI-6024E) was installed in the computer and connected to a 68-Pin Shielded Desktop Connector Block (SCB-68, MIO-16E Series) with a NI Cable (Type: SH6868). This combination allowed for several I/O ports to be configured to accommodate both thermocouple and function generator signals. Operation of the DAQ Card used is generally defined in Appendix-B with the pin specification list is further defined in the NI 6023E/6024E/6025E Family Specifications manual [45] .

Communication and data logging from the DAQ Card was conducted via a LabVIEW interface constructed specifically for this investigation. A virtual image (VI) (Appendix-C) was constructed to read and log the temperature signal from each of the thermocouples which sampled at a constant rate of $2 \mathrm{~Hz}$ throughout the duration of the thermal testing. Since the thermal portion of this investigation was to monitor LSP performance under the influence of temperature, steady-state setpoints were chosen and therefore in the interest of data reduction the sampling rate was kept low. Steady state operation was chosen due to limitations in the equipment, interest of time, lack of previous documentation and because transient thermal operation is beyond the scope of this investigation. External thermal effects on the operation of a laser have been relatively undocumented. External transient thermal effects imposed on a laser may have adverse effects on its operation as well, and additional testing is required and recommended to reveal its full influence.

The VI (Appendix-C) allowed for the LSP to be shaken at a wide range of frequencies within the test matrix between $0 \mathrm{~Hz}$ and $60 \mathrm{~Hz}$. Due to the weight of the assembly mounted on the oscillator and the limitations of the oscillator itself, amplitude variation was limited primarily at higher frequencies. The acceleration that can be applied to an object is a strong function of the mass of the object and the capabilities of the oscillator. Fundamentally, the acceleration of an object is defined by Newton's Second Law of Motion shown in Equation 3-1 below. 
When applying this equation to the operation of an oscillator, the force is considered to be the total amount of force that the oscillator can exert, and the mass is the sum of the test object mass and the mass of the oscillator's mounting piston. Applying this to Newton's Second Law yields Equation 3-2 which defines the acceleration of the payload as a function of the mass and the applied force from the oscillator.

$a=\frac{F_{t}}{m+m_{e}}$

The force that the oscillator can produce was a function of the cooling mechanism. The force ratings that this oscillator can exert can be found in Table 1. For this experimentation, a common computer cooling fan was adapted to mount to the oscillator's cooling passage. It should be noted that the addition of this cooling mechanism produced a slight increase in the applied force capability of the oscillator was observed.

Table 1: Force ratings for B\&K Vibration Exciter Type 4808

\begin{tabular}{|c|c|c|}
\hline & Cooled & Uncooled \\
\hline Force (lbf) & 42 & 25 \\
\hline
\end{tabular}

The total mass being oscillated varies based on the angle that the LSP is mounted, due to the difference in mass of the mounting adapter. These masses are shown in Table 2.

Table 2: Oscillated masses

\begin{tabular}{|c|c|c|c|c|c|}
\hline & $\mathbf{9 0}^{\circ} \mathbf{\&} \mathbf{0}^{\circ}$ adapter & $\mathbf{4 5}^{\circ}$ adapter & LSP & Hardware & Mounting Piston \\
\hline Mass (lbm) & 4.18 & 3.97 & 1.57 & 0.41 & 0.35 \\
\hline
\end{tabular}

Given the acceleration due to gravity, the maximum acceleration and total g-force were calculated for each condition, and are shown in Table 3. 
Table 3: Maximum acceleration and g-force calculations for every combination of testing parameters

\begin{tabular}{|c|c|c|c|c|}
\hline \multirow{2}{*}{} & \multicolumn{2}{|c|}{$\mathbf{9 0}^{\circ} \mathbf{\&} \mathbf{0}^{\circ}$ Adapter } & \multicolumn{2}{c|}{$\mathbf{4 5}^{\circ}$ Adapter } \\
\cline { 2 - 5 } & Cooled & Uncooled & Cooled & Uncooled \\
\hline Acceleration (ft/s $\mathbf{s}^{2}$ ) & 207.5 & 123.5 & 214.4 & 127.6 \\
\hline g-force & 6.4 & 3.8 & 6.7 & 4.0 \\
\hline
\end{tabular}

The accelerometer had a maximum measurement range of $\pm 2 \mathrm{~g}$. The maximum acceleration and g-force that the payload experienced in the $100 \%$ acceleration setpoints were unable to be confirmed and therefore the acceleration and gyroscopic characteristics of the payload were measured for the 50\% acceleration setpoint only. This limitation is further discussed later in Section 4.1.3. It is possible to estimate the acceleration parameters for frequencies that exceed the threshold limit of the accelerometer via non-linear interpolation however these parameters would only be estimates and therefore are not presented here. An estimation of the acceleration and g-force that the payload will experience is provided by $\mathrm{B} \& \mathrm{~K}$ Vibration Exciter Type 4808 and presented graphically in Figure 19. 


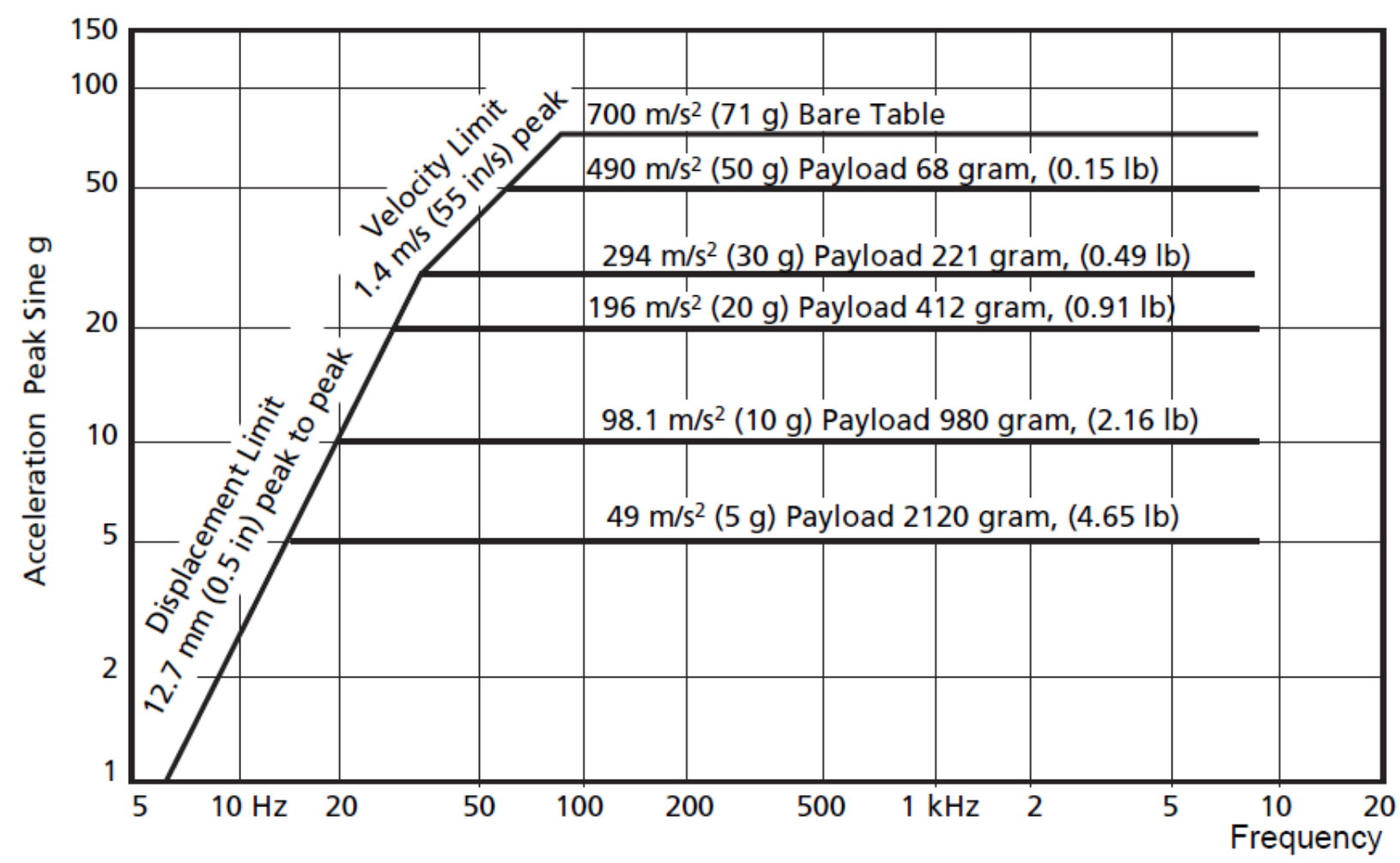

Figure 19: Performance curves for Vibration Exciter Type 4808 for operation without assisted cooling [41]

Communication with the UM6 Ultra Miniature Orientation Sensor for data logging was performed using a transistor-transistor logic (TTL) serial to universal serial bus (USB) connection interface. The interface converter was a USB 2.0 to TTL UART 6PIN Module Serial Converter CP2102, which converted the Serial TTL output from the accelerometer to a USB 2.0 input. Initial calibration and graphical display was performed on the accelerometer using the accompanying freeware CH Robotics (CHR) Serial Interface Software v2.2.0. Data logging was conducted with the CHR interface (Appendix-C), which was designed to split the serial output signal from the accelerometer and extract the acceleration components.

\subsection{Laser}

High power transmission through fiber optic cables is a research area of great importance that has the potential to revolutionize an industry. The fiber transmission energies required to achieve optical breakdown intensities of $\sim 100-200 \mathrm{GW} / \mathrm{cm}^{2}$ are however extremely difficult to achieve at the fiber output in a harsh engine environment [8,9]. For this current ignition 
strategy, each cylinder of the engine will be equipped with its own ignition laser instead of a different fiber optic cable transmitting high peak pulse energy for each cylinder. This strategy is different from alternative methods such as using a single ignition laser for the entire engine whose high pulsed energy is multiplexed through fiber optic cables to the respective cylinder. In this experimental setup, only a single LSP was subjected to the oscillatory and thermal perturbations for output analysis.

Regardless of the strategy used for introducing the ignition pulse into the combustion chamber, a sufficient means of pumping the laser is necessary. In this work, the pump energy exits a fiber-coupled diode laser and is directed to the LSP that is directly mounted on the engine. The pump laser is significantly larger and heavier than the ignition laser and is far more susceptible to heat and vibration as well. For this reason, the pump laser was remotely located from the engine. Ideally, the pump and the ignition laser would both be small, compact, coupled together and directly mounted on an engine. LI technology has reached the point where a single compact unit may be produced, however financial limitations are still of concern.

\subsubsection{Laser Testing Equipment}

\subsubsection{1. $\quad$ Laser Spark Plug}

The LSP used for this line of testing was a miniature diode end pumped passively qswitched Nd:YAG laser operating in the infrared (IR) at a wavelength of $1064 \mathrm{~nm}$ [40]. Figure 20 shows a basic optical alignment diagram of the high reflector (HR), Nd:YAG Rod, q-switch and OC which comprise the construction of the LSP. This design was chosen in part from the work of McIntyre et al. who performed an investigation of a lasing medium's concentration on the output characteristics of a passively q-switched LI system. The work showed that by lowering the Neodymium concentration in the active media, lowering the OC reflectivity and reducing the initial q-switch transmission, a larger and brighter spark can be achieved [21]. 


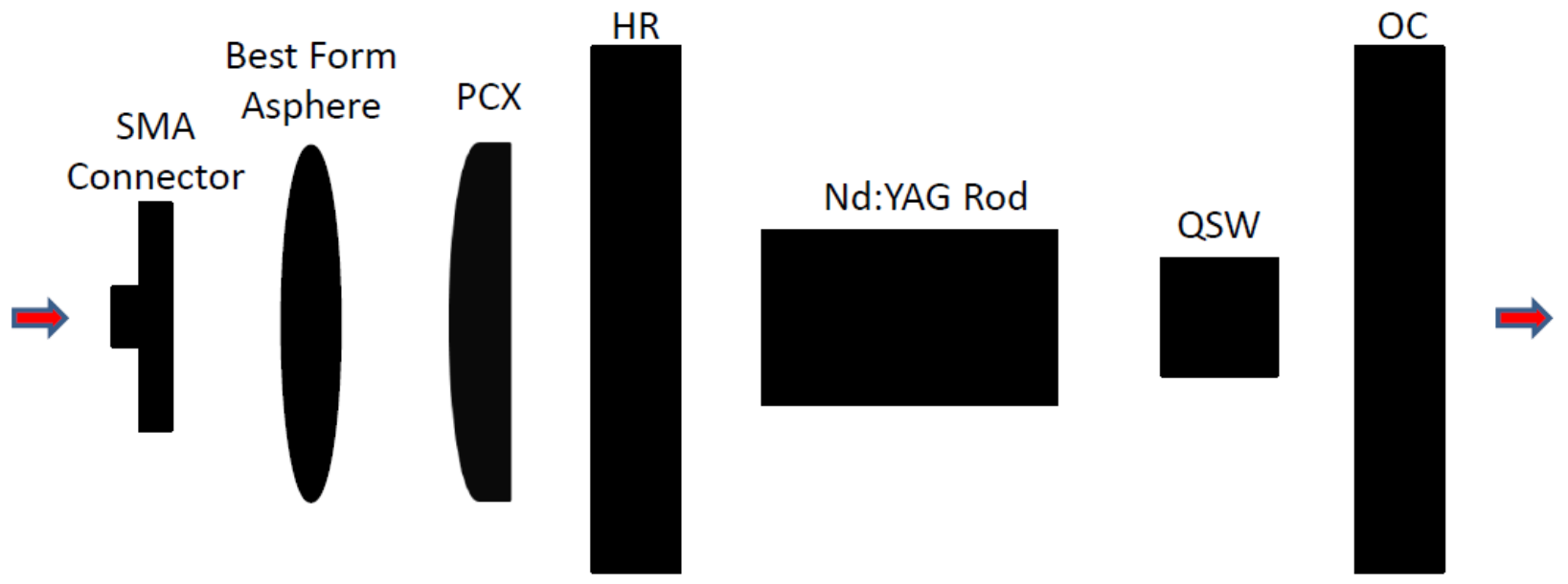

Figure 20: Basic layout and construction of the LSP

The addition of a q-switch to the laser cavity provides a means of modulating the energy storage capacity of the cavity. This is done to produce a pulsed output from the ignition laser for the sole purpose of providing the high peak power output necessary for generating sparks as previously discussed in Section 2.4.1. By inserting a q-switch into the laser cavity, the energy storage capability can be greatly increased. When the q-switch has absorbed enough photon energy to reach its threshold, as previously discussed in Section 2.4.1.2, it becomes bleached and the high potential buildup of energy is released from the laser cavity thereby creating a high peak power output pulse [21]. As previously shown in Figure 6, the output power of a q-switched pulse is typically several orders of magnitude larger than a $\mathrm{CW}$ output.

The approach of using a single laser to initiate the onset of combustion was investigated as a solution to the problems encountered with transmitting high peak power into and through fiber optic cables. The LSP is capable of producing pulsed output energies in the megawatt range which are steered directly into the combustion chamber from the ignition laser which is mounted directly onto the engine. This strategy alleviates the problems that arise from high power transmission through fiber optic cables of open beam transmission. The LSP is designed to be much more impervious to the engine's harsh environment than previous laser based designs for ignition applications. The preliminary design that was previously shown in Figure 14 is much larger and heavier than the LSP that would be designed for mass production. The preliminary design weighs $2 \mathrm{lb}$. $6.9 \mathrm{oz}$. and is approximately $12 \mathrm{in.}$ long with a 4 in. diameter. This design was initially chosen because it utilizes common off the shelf components that are 
readily available and at a relatively low cost. The configuration of this design is presently considered to be the geometrically simplest design using the fewest components necessary to achieve optical breakdown. The size, shape and construction of the present LSP prototype was manufactured with adjustability in mind. Each of the optical components are physically separate from each other to allow for interchangeability and maintenance to provide the necessary isolation to determine the optimal characteristics of the LSP for this application. It is possible for the optical components used here to be reduced into a monolithic design. The final product has the potential to be significantly reduced in size and mass to approximately the same size and mass of a conventional spark plug.

\subsubsection{Power Meter, Oscilloscope, Detector Head and Photodiode}

Measurement of the LSP's output parameters was critical for evaluating the LSP's performance under the influence of temperature and vibration. With the PE50BB-DIF detector head placed in the output beam, it was connected to the Laserstar Power Meter to obtain the laser energy output. The measurement location of the detector head varied in this testing for reasons further discussed in Section 5.5. The power meter was in turn connected to the computer for real time data logging capabilities via the power meters accompanying software. Laser pulse energy was collected and recorded at each test matrix setpoint.

A High Speed Photodiode Detector (DET110) was used to monitor the pulse width, pulse width variation, q-switch delay and jitter of the LSP. It was mounted at an angle pointing directly at the power meter's detector head and connected to an appropriate oscilloscope. The reflection of the pulsed laser light off of the detector head's diffuser was captured by the photodiode and recorded by the oscilloscope. With the power meter detector head and photodiode in place, the output parameters of the LSP were measured and recorded.

A mounting bracket with a vibration isolation pad was constructed to hold the detector head and the fast photodiode. This setup is shown in Figure 21. The signal from the detector head was analyzed by the power meter through RS232 serial communication and logged into the computer via the accompanying software from OPHIR. The signal from the photodiode was read by the oscilloscope which was able to detect the pulse duration as well as the pulse 
frequency of the LSP. Data and specification sheets for the detector head, power meter, oscilloscope and photodiode may be found in Appendix-B.

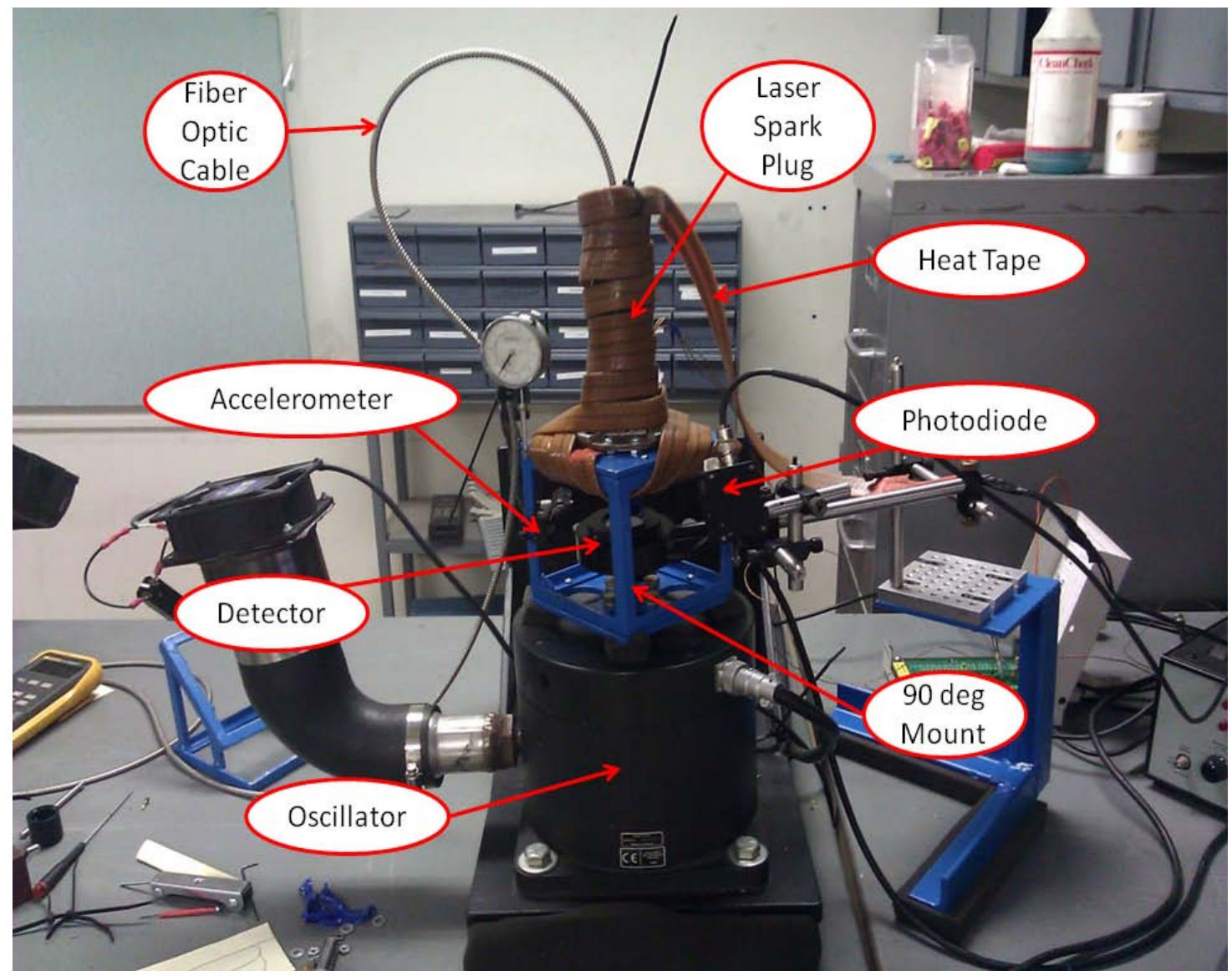

Figure 21: Positioning of the equipment in the experimental setup

\subsubsection{Pump Laser}

The laser used to pump the ignition laser was quasi CW diode laser bank operating at 808 $\mathrm{nm}$. This fiber-coupled diode laser was used to end pump the primary ignition laser with $500 \mu \mathrm{s}$ duration pulses at 200 watts [40]. These parameters were chosen based on review of the literature and the previous experimentation of Woodruff et al. For mass production applications, the pump laser would remain remotely located from the engine and be multiplexed through a series of fiber optic cables. Each fiber would then transfer the light energy to the LSP mounted on the respective cylinder. Experimentation conducted by Yalin et al. illustrates a means of accurately multiplexing a low energy laser pulse for ignition purposes $[7,8,9]$. A description of 
how a multiplexed strategy is applied to a LI setup can be found in the literature and extends beyond the scope of this experimental setup and procedure.

The advancement of laser technology has developed to the point where several components comprising the construction of a laser can now be manufactured to suit an alternative pumping strategy. Advancement in semiconductor research have allowed for the development of a Vertical-Cavity Surface-Emitting Diode Laser (VCSEL) that can be manufactured at a much lower cost, smaller and less sensitive to high temperatures than previous edge or surface emitter diode pumps [46]. If implemented properly, these diode lasers have the potential to act as a miniaturized end pump source for q-switched lasers. This technology has yet to develop for LI applications, however it has been applied as a pumping mechanism for end pumped, Nd:YAG solid-state lasers [47, 48, 49].

\subsection{Design of the Experiment}

The experimental design used in this testing was constructed based on a thorough literature review and a preliminary investigation of the targeted parameters. From these sources, a set of independent and dependent variables were developed to display the gathered results in a manner that may be advantageous to a wide range of disciplines. The independent variables were temperature, vibrational frequency, acceleration and mounting angle of the LSP, while the dependent variables were pulsewidth, pulse width variation, q-switch pulse delay, jitter and output energy. A four dimensional array of three cubic mounting orientation matrices were used whereby each setpoint has five test values. Execution of the test matrix is further discussed in Section 4.1.4.

For each test value in the matrix the pulse width, pulse width variation, q-switch pulse delay, jitter and energy output values were gathered and recorded. The testing procedure at each of the setpoints was conducted a total of three times so that an average resulting value could be calculated. A more accurate result may be obtained with a higher number of repetitions, however, for the preliminary investigative purposes of this study it was deemed unnecessary. For each of the resulting dependent variables collected, one standard deviation was reported for

each of the data points. This variation interval was chosen to gauge the deviation of the collected data from the mean. Since the resulting dependent variables are independent of time, day-to-day 
variation of the collected data is expected to be minimal. However to verify the negligibility and to reduce the error of the results, the test matrix was repeated three times for each data point as an attempt to account for this variation. This is further discussed in Section 4.1.4.

\subsection{Sources of Error}

As with every experiment, there is a certain amount of error associated with the data collection and ultimately the results. Regardless of the precision in which the experimental matrix is executed, there is an inherent amount of error in the equipment used as well as the inevitable human error. In this experimentation, an attempt to minimize the error from human interaction and day-to-day variation was performed by repetition of the test matrix and statistical analyses to obtain a data set that was globally representative of the setup's performance. The following are the defined sources of error involved in the equipment used in this preliminary testing as specified by the manufacturers.

\subsubsection{NI_DAQ SCB68 Shielded Desktop Connector Block}

There are several sources of error involved in SCB68 Shielded Desktop Connector Block. Compensation error from the SCB68 can arise from the temperature sensor wires and the screw terminals on the block. This presents a resistance node in the measurement technique, which may yield results that vary up to $\pm 1.8^{\circ} \mathrm{F}$ [50]. An attempt was made to minimize this error by keeping the connector block away from the heaters and isolated from the transient vibrational effects from oscillation propagation through the table and floor.

\subsubsection{NI DAQ Card (PCI-6024E)}

Measurement error from the DAQ device itself comes from inaccuracies in the preset gain and offset of the device and from ambient noise as well. The service manual for the DAQ card provides calculations for a means of calculating the accuracy of the device and provides a procedure for calibrating the device as well. The DAQ system was calibrated with a NISTtraceable, independently calibrated and cold junction compensated Fluke 714 Thermocouple Calibrator that is specified to have an error of $\pm 0.6^{\circ} \mathrm{F}$ [51]. The calibration procedure was followed using the WVU Standard Operating Procedure (SOP)-0381 Thermocouple Measurement Verification worksheet in accordance with 40 Code of Federal Regulations (CFR) 
1065.315. Potential error exists with the linearization of the calibration. This is dependent upon the degree of the polynomial used for the best fit line. As a consequence, the polynomials used are considered to be approximations of the actual thermocouple output. For each thermocouple calibrated, a linear regression was preformed for each of the calibrated points yielding an $\mathrm{R}^{2}$ value of $\geq 0.998$. This conforms to the regulations set forth by the CFR and therefore no additional error was accounted for in the linearization calibration. Error potentially exists during this calibration process from the thermocouple wire connection to the junction block as well. This is accounted for in the error that arises from the thermocouple and thermocouple wires presented in the next section.

\subsubsection{Omega Cement-On J-Type Thermocouples}

The thermocouples used to make the temperature measurements were Omega Cement-On J-Type Thermocouples Style 3. They were fast response thermocouples that are designed for surface temperature measurements. Inconsistencies, inhomogeneities and impurities in the thermocouple wire resulting from the manufacturing process were the main sources of error in the thermocouple construction. As specified by the manufacturer, the total error resulting from these main sources were $2{ }^{\circ} \mathrm{F}$ or $0.4 \%$ whichever is greater [52]. Given the temperature range of this experimentation, the error assumed was $2{ }^{\circ} \mathrm{F}$ as this value was always greater than $0.4 \%$.

\subsubsection{UM6 Ultra Miniature Orientation Sensor}

The accelerometer used in this investigation was a UM6 Ultra Miniature Orientation Sensor. The acceleration signal extracted from the sensor was processed to obtain instantaneous acceleration measurements at each of the test points. Calibration of the accelerometer and gyroscope was performed using the CHR Serial Interface provided as freeware from the manufacture website. The manufacturer specifies that the major influence affecting the measurement is thermal variation. The acceleration sensor had a sensitivity change due to temperature of $0.02 \% /{ }^{\circ} \mathrm{F}$. As a result, the accelerometer was mounted away from the heat tape. Due to the rigid construction of the adapter to mount the LSP on the oscillator, any transfer functions that may occur were assumed to be negligible. This was verified by using multiple installation locations prior to testing. 


\subsubsection{Vibrational Setup and Control}

There was a certain amount of error that was inherent in the vibrational setup, control parameters and equipment. Sources of error reside in the DAQ system, amplifier and vibration exciter itself. It was possible to calculate the acceleration that the payload can oscillate at as a function of its weight and input signal, however the damping system that was implemented for a means of vibration isolation introduces a source of error that is very complex to calculate. The damping coefficient of the open cell vibration isolation pad was a linear time invariant component which changed with payload frequency and the natural harmonic frequency of the oscillator. The acceleration at which the pay load was actually oscillating at was verified by the accelerometer. As shown in Figure 22, the difference between the calculated data and the response from the accelerometer takes a similar form but differences in magnitude are evident. This may be due to the fact that the mass is assumed to be uniform in nature and does not account for any geometric constraints. For the purposes of this work, the accelerometer response frequency is presented as the actual oscillating frequency.

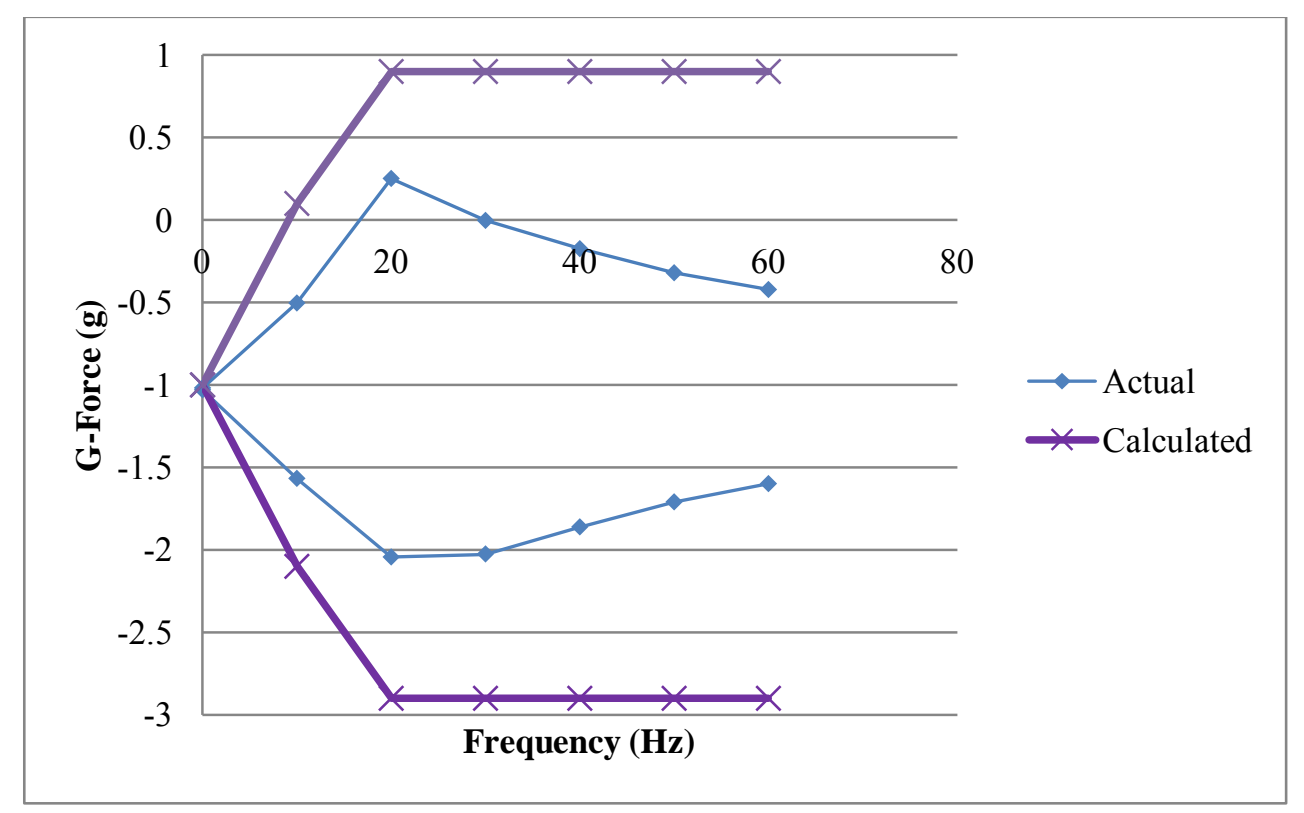

Figure 22: Calculated and actual 50\% x-axis g-force of the payload in the 90 degree mounting orientation as a function of frequency 


\subsubsection{Temperature Setup and Control}

The LSP was wrapped with the heat tape and allowed to come to an equilibrium temperature for each thermal setpoint. This strategy for controlling operating temperature presents an inherent source of error since most of the heat transfer is conductive. This is different from what the LSP would experience on the engine since for this design the LSP was mounted at a suitable distance from the head where the majority of the heat transfer would be convective and radiative. This conductive heating strategy leads to non-uniform temperature distributions within the laser as shown in Figure 23. There is a significant difference in the optical component operating temperature for a given thermal setpoint. The 90 degree mounting orientation for example acts as a heat stack where the applied heat was funneled up the optical axis of the LSP effectively making the highest point of the laser the hottest. Here in this orientation, the connector was the highest point of the laser and the $\mathrm{OC}$ was the lowest.

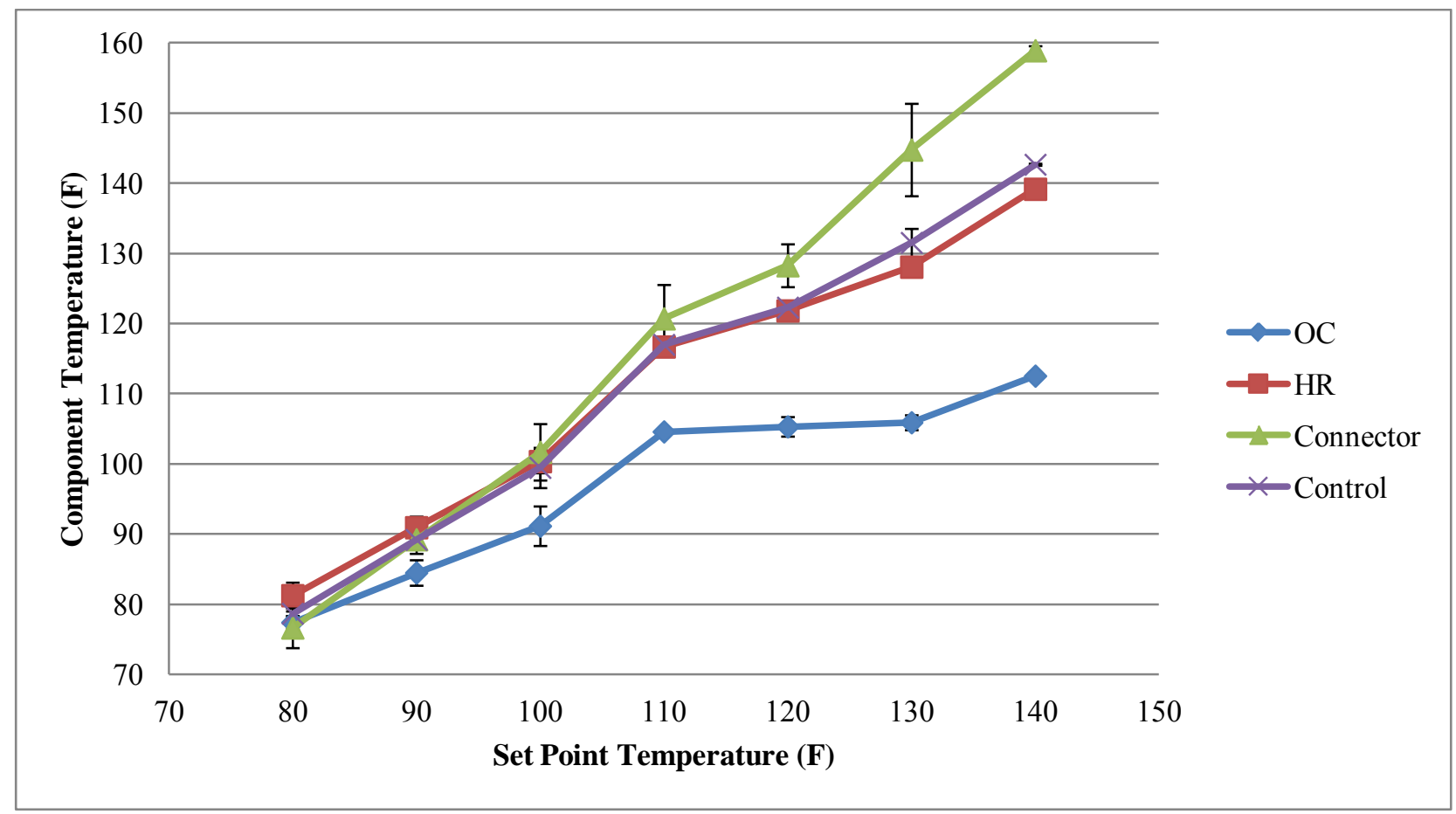

Figure 23: Laser spark plug component temperature as a function of setpoint temperature in the 90 degree mounting orientation 


\subsubsection{Total Error}

There are several independent parameters that may affect the error in the measurements, however only the calculated error measurements as specified by the equipment manufacturers are presented here. The total error involved in the temperature measurements due to the equipment was calculated to be $\pm 4.3^{\circ} \mathrm{F}$, and the total error involved in the oscillation measurement was calculated to be $\pm 0.5 \mathrm{~Hz}$

\subsection{Safety}

Safety is a major requirement regardless of the situation you are presented with. When performing tasks that have the potential to cause damage to your sense of vision such as the LSP, the proper precautions must be taken to ensure safety. For the purposes of this preliminary investigation, safety precautions included wearing the proper safety glasses, fully enclosing the setup to the point where no light can escape and posting the proper warning signs notifying others of the hazards.

The LSP assembly is considered a Class 4 optical source capable of achieving upwards of $3 \mathrm{MW}$ of peak pulse power. A Class 4 laser is the most dangerous class of laser. The class of laser is dependent upon the laser output power and wavelength and is a means of generally classifying the lasers ability to cause harm. A Class 4 laser has the capability to burn the skin and cause permanent damage to vision even if exposure to the laser beam occurs through reflection or diffusion [53]. These lasers also have the capability to ignite combustible materials and therefore a significant amount of care must be taken in controlling the path of the beam. The categorization of every laser is defined by the American National Standards Institute (ANSI) Z136.1 Standard (Z136.1-2000) and is typically regulated in the work place by the Occupational Safety and Health Administration (OSHA) [54]. For compliance and verification purposes for these rules and regulations, the LLNL Laser Safety HS5200-W test was completed to ensure knowledge of safety during this testing.

To make certain that personal were properly notified of the dangers accompanying this optical setup, signs were posted with the operating parameters of each of the lasers as shown in Figure 24. Since the test cell used in this investigation was not primarily constructed with optical testing in mind, precautionary measures such as blocking out all the windows and 
covering all highly reflective surfaces were taken. Coworkers involved in alternate projects in the same facility were supplied with literature explaining the dangers of laser exposure and were lectured about the proper techniques and safety precautions necessary to ensure their own personal safety. For the duration of this testing, only a single researcher was in the test cell while the laser was operating. Since safety was a major concern in this experimentation and the proper personal protection equipment (PPE) were used to protect against the hazards of laser exposure.

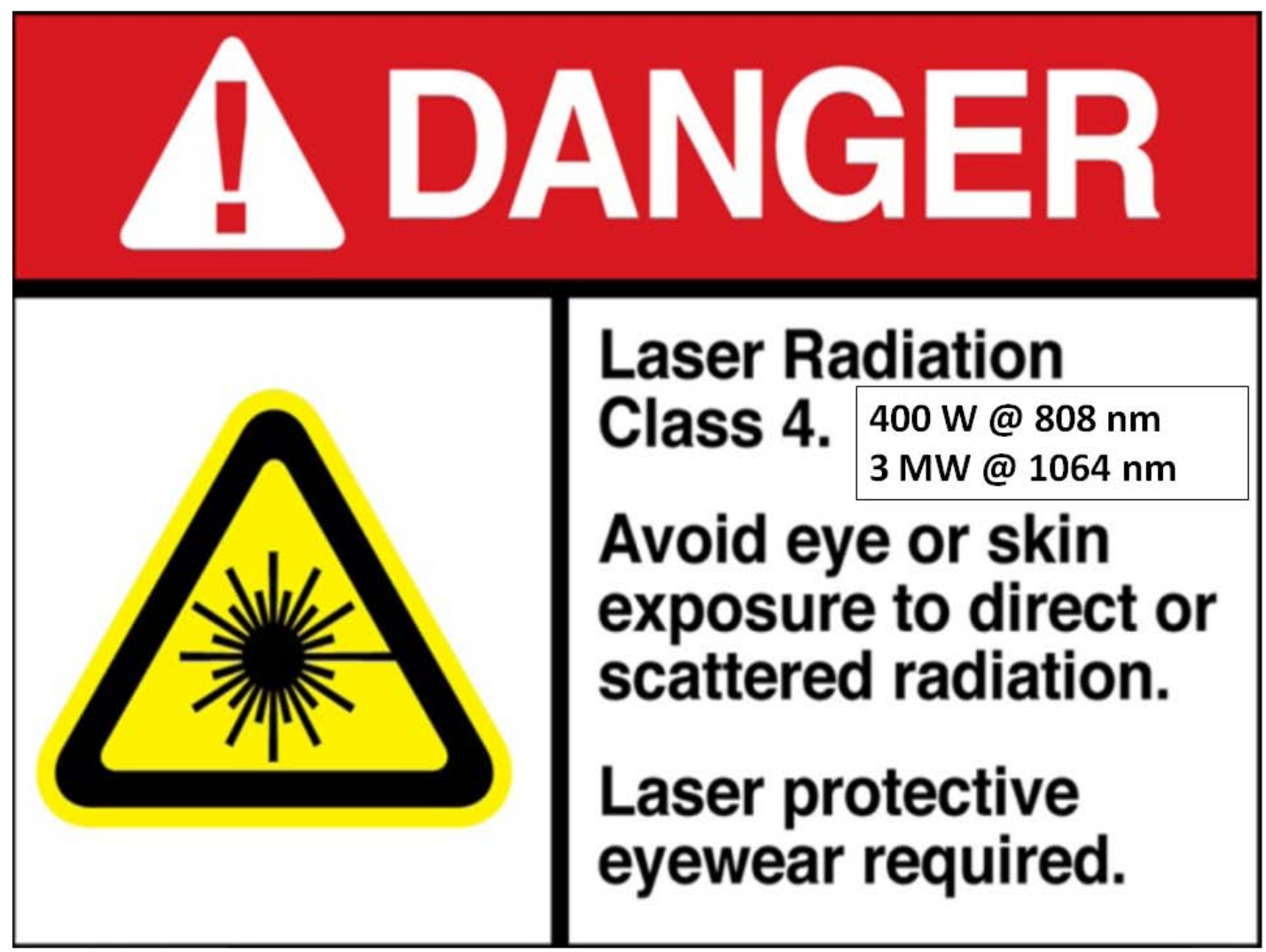

Figure 24: Danger sign posted in the optical test cell 


\section{EXPERIMENTAL PROCEDURE}

\subsection{Diode laser and LSP}

To provide the best means of comparison between the results gathered here and previous experimentation conducted by NETL, the settings of the diode laser and the LSP were set to the settings as specified by McIntyre et al. [29]. Due to time limitations, only the pump pulse width and pump pulse frequency were varied throughout the testing. The optical components comprising the cavity of the LSP were a Cr:YAG passive q-switch with a $10 \%$ initial transmission, an $\mathrm{OC}$ with a $20 \%$ transmission, a Nd:YAG gain medium with a doped concentration of $0.9 \%$ and a HR with a $99.999 \%$ reflectivity. The diode laser was water cooled at $82.4^{\circ} \mathrm{F}$ using a chiller and operated with a variable pulse width throughout the test matrix as a function of setpoint temperature. The thermal distributions were different for each of the mounting angles and for this reason, the pump pulse width was adjusted to be approximately 125 $\mu$ s less than the pump pulse width that would initiate a second consecutive output pulse. The pump pulse widths used for each mounting orientation and temperature are presented in Table 4. Note that for each mounting orientation, the pump pulse width increased as a function of setpoint temperature, and the rate at which the pump pulse width increased was smallest for the 45 degree mounting orientation. This relationship is further explained in Section 5.2.3.

Table 4: Pump pulse width used for each thermal and mounting orientation setpoint

\begin{tabular}{|c|c|c|c|}
\hline & \multicolumn{3}{|c|}{ Pump Pulse Width $(\boldsymbol{\mu s})$} \\
\hline Temperature ( $\left.{ }^{\mathbf{}} \mathbf{F}\right)$ & $\mathbf{0}$ Degree & 45 Degree & 90 Degree \\
\hline $\mathbf{8 0}$ & 350 & 375 & 375 \\
\hline $\mathbf{9 0}$ & 380 & 375 & 390 \\
\hline $\mathbf{1 0 0}$ & 390 & 375 & 390 \\
\hline $\mathbf{1 1 0}$ & 440 & 380 & 410 \\
\hline $\mathbf{1 2 0}$ & 450 & 380 & 430 \\
\hline $\mathbf{1 3 0}$ & 500 & 380 & 490 \\
\hline $\mathbf{1 4 0}$ & 540 & 390 & 540 \\
\hline
\end{tabular}


Since the q-switch delay of the LSP is a function of the power and time that the pump laser is exciting the active medium, at some setpoints multiple $1064 \mathrm{~nm}$ output pulses occurred. This especially happened at some of the higher frequency and thermal setpoints where jitter was large. Referring back to Figure 6 which shows the strategy for obtaining high peak power pulsed output with a passively q-switched laser, as pump energy enters the cavity, the q-switch absorbs the pump energy until it reaches it saturation threshold bleaches. This bleaching effect only occurs for a short amount of time until the ions return to their ground state and the majority of the $1064 \mathrm{~nm}$ energy has left the cavity. If the pump pulse width is long, the q-switch will saturate, dump the $1064 \mathrm{~nm}$ energy and begin again to absorb the $808 \mathrm{~nm}$ energy for the remaining pump pulse duration. If the pump pulse width is too long, the q-switch may reach its saturation threshold multiple times for a single pump pulse leading to multiple output pulses. Multiple output pulses are reported by the power meter as a single pulse since the energy measurement is triggered from of the leading edge of the pump pulse. When multiple $1064 \mathrm{~nm}$ output pulses occur within a single pump pulse, the resulting output energies are additive and are reported by the power meter as a single pulse. When continuously pumped, the passively qswitched laser will produce periodic output where the repetition is set by the pump rate and the combination of initial q-switch transmission and OC. For the setpoints where multiple ignition pulses occurred, the repetition pulses were negated and the average energy presented here was adjusted accordingly.

The frequency of the pump pulse varied as a function of the oscillatory frequency of the

payload. Since the oscillatory frequency of the payload was intended to imitate the rotational frequency of the crankshaft from a four stroke IC engine, the frequency of the pump source was triggered at exactly half the frequency of the oscillatory setpoint. This was done to coincide with the fact that for a four stroke IC engine, ignition occurs once every two revolutions of the crankshaft.

\subsubsection{Alignment}

Alignment is critical due to the necessity for pinpoint accuracy for optical cavity operation. Improper alignment of the pump laser steering optics may cause the pump energy to only reach a portion of the gain media. If the pumping beam from the diode laser reaches the 
$\mathrm{Nd}$ :YAG laser cavity but is not properly coupled with the high reflector and Nd:YAG rod, little or no1064 nm output energy will be emitted from the laser.

The alignment of the laser not only influences the output energy of the laser but it also influences the q-switch delay. In an ideal case, the pump light would excite the gain media in such a manner that constant and homogeneous stimulated emission would occur. With a slight misalignment, the light does not effectively couple with the gain media yielding a variation in cavity resonance resulting in a longer time duration to reach q-switch saturation. When aligning this laser, the q-switch delay was minimized through an iterative process of adjusting all available degrees of freedom. Once a minimum q-switch delay was obtained, further minute adjustments to the alignment were made to optimize the output energy. Great care was taken when aligning and optimizing the LSP. This alignment procedure was repeated several times during testing.

The initial optical alignment procedures were performed with the q-switch removed and using a $633 \mathrm{~nm}$ Helium-Neon laser which provided the best results for visual alignment of the LSP based on the given back reflection of the intra-cavity components. Once a satisfactory alignment was achieved based on back reflection with the Helium-Neon laser, the q-switch was reinstalled and the LSP was connected to the diode pump source. At this point, the optimization process previously discussed was performed in order to minimize the delay of the passive qswitch and to maximize the energy at this point. With the LSP fully aligned and properly operating, it was mounted on the oscillator and wrapped in heat tape to be ready for testing when needed.

\subsubsection{Temperature}

Temperature control of the LSP was monitored and modified by the J-type thermocouples and the Omega temperature controller. With other thermocouples mounted on the laser, a thermal profile of the LSP was gathered. A thermocouple was mounted on the SMA connector head of the coupled fiber of the diode laser, another was mounted on the adjustable housing of the high reflector and the third was mounted on the housing for the OC. The HR and OC mounting locations were chosen due to spatial limitations of the LSP assembly. Ideally, the thermocouples would be mounted directly to the HR, OC, q-switch and Nd:YAG rod; however, 
this was not possible with the current optical assembly. For each thermal setpoint, a stabilization period was allowed to negate any transient effects. The heat tape temperature was controlled to the specified setpoint temperature, and the resulting thermal profile of the LSP was measured and recorded through the DAQ system and LabVIEW interface.

\subsubsection{Vibration}

With the LSP securely mounted on the oscillator, vibration was applied to the LSP. Frequency and applied acceleration were varied in accordance to the test matrix at each vibrational setpoint via the LabVIEW vibrational interface. To verify the acceleration of the payload, the accelerometer was mounted on the adapter and connected to the computer as a confirmation measure. Linear acceleration and rotational acceleration were measured and recorded for the three primary Cartesian coordinate axes yielding a total of six movement measurements for a single setpoint.

Due to the sensitivity of the surrounding equipment needed to conduct this investigation, great care was taken to isolate the vibration. The oscillator itself was placed on an open-cell foam isolation pad on a rigid steel table which kept the transfer of vibrational energy low. Rubber damping pieces were placed under each of the legs of the table as to minimize any vibrational energy transfer to the rest of the room as well. Upon review of the operations manual for the detector head, it was found that the detector head was extremely sensitive to vibration [55]. For this reason, open-cell foam was also placed under the mount that was constructed to hold the detector head and photodiode as an additional damping mechanism. With all the vibration isolation precaution in place, the oscillatory component of the test matrix was conducted.

Accurate vibration control of the LSP is a crucial aspect in obtaining meaningful results in this experimentation. In an effort to ensure proper oscillatory response, two measures were implemented to monitor the vibration. The input signal from the computer to the oscillator was monitored and recorded from the graphical output on the LabVIEW interface and from an oscilloscope which displayed the signal output from the SCB68 Connector Block to the oscillator as well. The acceleration response signal from the oscillator was monitored with the accelerometer which was mounted on the adapter plate as shown in Figure 25. As previously 
discussed, this mounting location was chosen because the accelerometer is thermally sensitive. Both the oscillatory position input and the acceleration response signal from the accelerometer were logged via the DAQ system and LabVIEW interface. The input sine wave from the DAQ system was used in defining the frequency setpoints and was monitored using an oscilloscope. The induced g-force was normalized at each setpoint for consistency purposes throughout the test matrix.

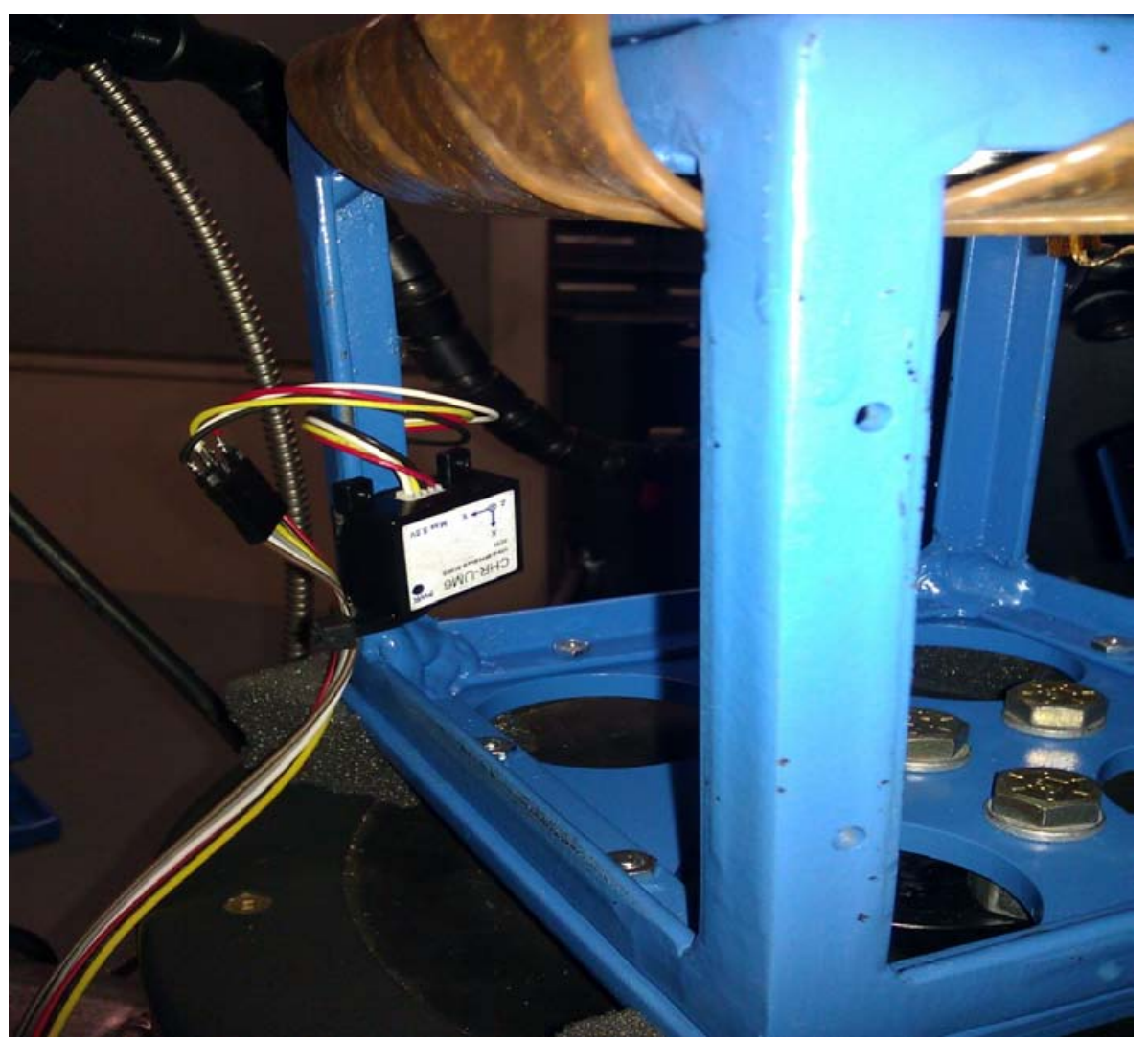

Figure 25: Accelerometer mounting location for the 0 and 90 degree mounting angles

Prior to conducting the test matrix, the payload was vibrated to quantify the maximum gforce that the oscillator could apply to the payload. The accelerometer could only measure accelerations of $\pm 2 \mathrm{~g}$, therefore an alternative means of calculating the g-force setpoint was necessary since the calculated maximum g-force from Table 3 exceeds this limit [42]. For the higher g-force setpoints, the induced g-force could not be verified. Upon examination, it was discovered that the oscillator amplifier gain varied linearly with induced acceleration of the payload. Therefore for each frequency and mounting angle setpoint, the maximum g-force setpoint was determined by increasing the gain of the oscillator's amplifier until the system 
reached the upper current limitation of 9 amps. The maximum amplifier gain required to reach this current limitation defines the maximum g-force setpoint in the test matrix and is tabulated below in Table 5. The results from the amplifier gain to maximum g-force correlation loosely coincide with the specified maximum acceleration ratings of the oscillator as previously shown in Figure 19 which is as to be expected.

Table 5: Amplifier gain needed to reach maximum (100\%) g-force of the payload for each orientation mounting angle as a function of frequency

\begin{tabular}{|c|c|c|c|}
\hline Frequency (Hz) & Gain: 0 Degree & Gain: 45 Degree & Gain: 90 Degree \\
\hline $\mathbf{1 0}$ & 4.2 & 4.4 & 5.0 \\
\hline $\mathbf{2 0}$ & 5 & 6.0 & 6.6 \\
\hline $\mathbf{3 0}$ & 6.2 & 6.2 & 6.8 \\
\hline $\mathbf{4 0}$ & 6.2 & 6.2 & 6.8 \\
\hline $\mathbf{5 0}$ & 6.4 & 6.2 & 6.5 \\
\hline $\mathbf{6 0}$ & 6 & 6.0 & 6.4 \\
\hline
\end{tabular}

The capabilities of the oscillator were fundamentally limited by the mass of the payload. As frequency increased, the maximum amplitude that the oscillator can produce decreased in a nonlinear fashion. This nonlinear response is displayed in Figure 26 where the greatest amplitude capable of being produced by the oscillator occured at lower frequencies. As applied frequency was increased, the maximum capable amplitude of the oscillator declines. This directly relates to the calculated and measured g-force as previously shown in Figure 19 and Figure 22 since induced g-force is not only a function of payload mass, but is a function of the operational frequency and amplitude as well. 


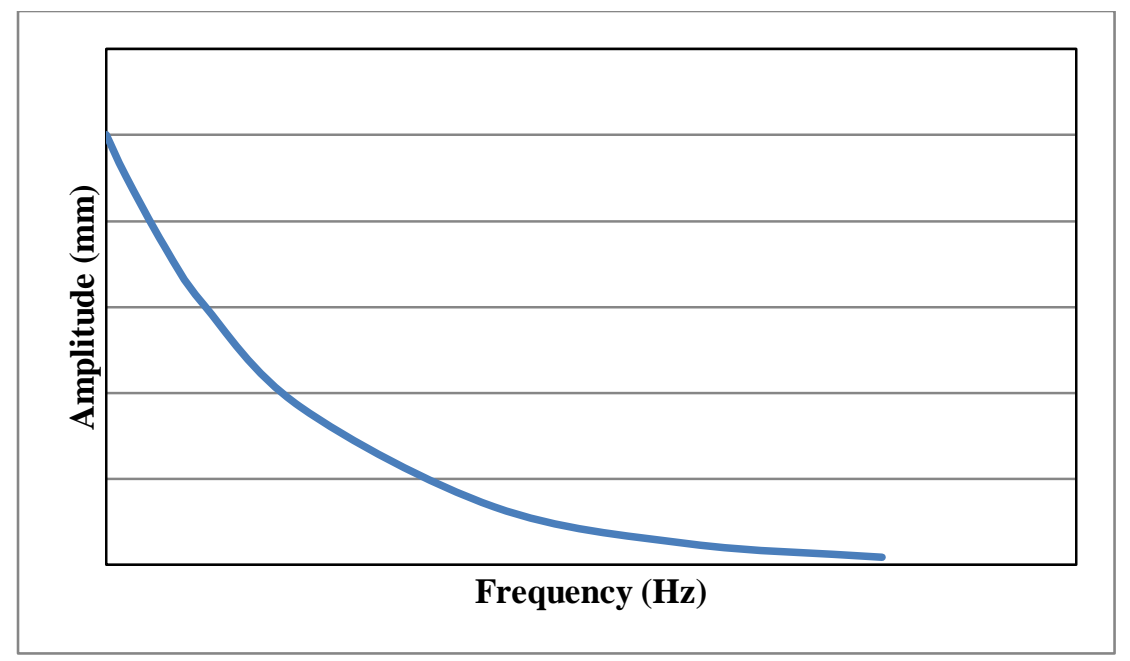

Figure 26: Generalized amplitude response of an oscillator as a function of frequency

Acceleration was normalized as a function of the amplifier gain as previously defined in Table 5, where the 50\% acceleration setpoints represents exactly half of the maximum amplifier gain and the $100 \%$ acceleration setpoints represent $100 \%$ of the maximum amplifier gain. This normalization was performed as a result of the nonlinear amplitude response as a function of applied frequency shown in Figure 26.

\subsubsection{Test Matrix}

The testing template was a four dimensional array comprised of three cubic matrices. Each of the matrices in the array was conducted at the three different test angles: $0^{\circ}, 45^{\circ}$ and $90^{\circ}$ relative to the horizon. In each matrix, temperature, frequency and percentage of maximum gforce were varied. Temperature was varied from $80^{\circ} \mathrm{F}$ to $140^{\circ} \mathrm{F}$ in $10^{\circ} \mathrm{F}$ increments giving a total of seven thermal setpoints. Vibrational frequency was varied from $0 \mathrm{~Hz}$ to $60 \mathrm{~Hz}$ in $10 \mathrm{~Hz}$ increments giving a total of seven frequency setpoints which represent engine speeds from 0 RPM to 3600 RPM in 600 RPM increments. Acceleration was varied between $50 \%$ and $100 \%$ of the maximum total g-force capability of the oscillator as a function of mounting angle.

The test matrix shown in Figure 27 is a cubic array of setpoints for a single mounting angle. Each setpoint for each mounting angle was conducted a minimum of three times as to obtain an average distribution of the resulting dependent parameters. For each set of data at a given setpoint, the standard deviation was calculated and used as a measure of error for the graphs presented in Section 5. 


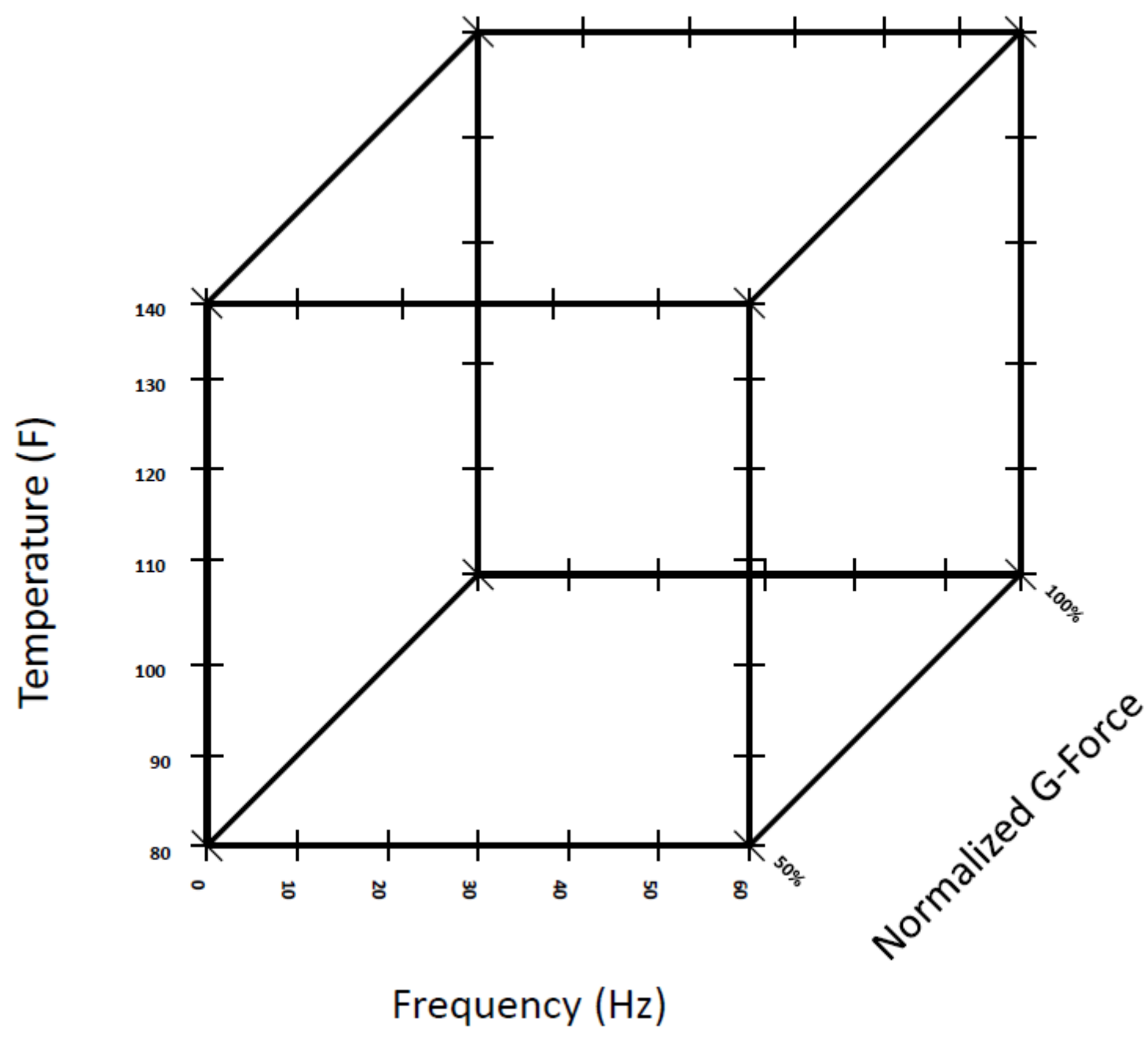

Figure 27: Test matrix for a single mounting angle

Using the results gathered from the first test matrix, a better means of conducting the data collection process was used to obtain increasingly more accurate results. Figure 28 below shows the complete array of test matrices for each mounting angle. The resulting effects that the independent variables had on the dependent variables were found to be quite significant and are presented in the following chapter. 


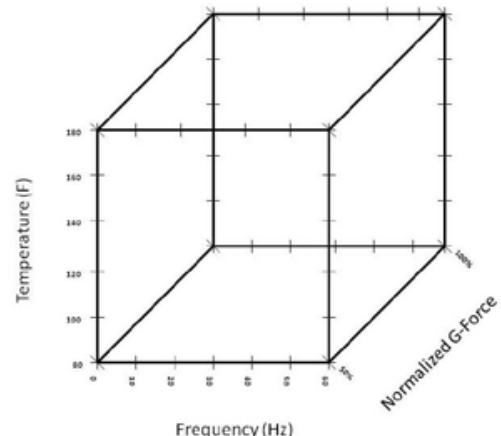

Frequency $(\mathrm{Hz})$

$0^{\circ}$

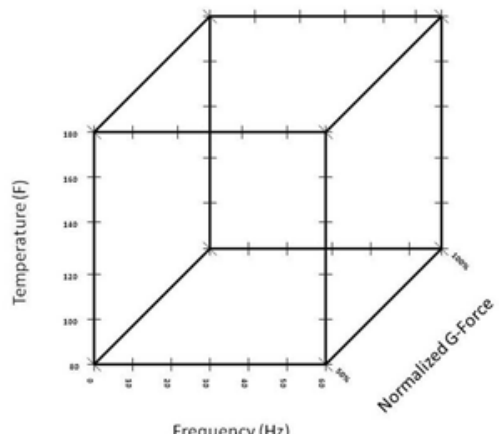

$45^{\circ}$

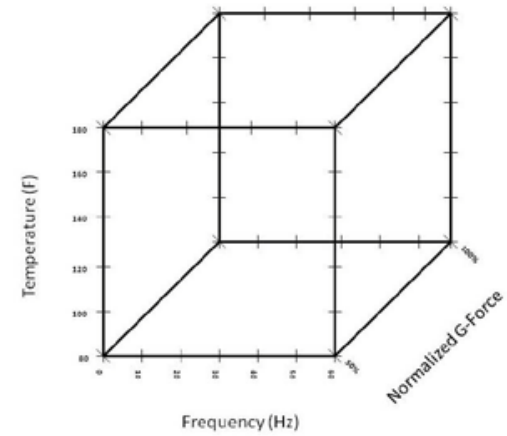

$90^{\circ}$

Figure 28: Four dimensional array of three cubic matrices

The test matrix was conducted as follows. For a given mounting angle, the setpoint temperature was applied and allowed to reach a steady-state. It should be noted that it takes approximately 45 minutes for the temperature of the LSP to reach equilibrium. For each thermal setpoint the LSP was subjected at two different levels of g-force, $50 \%$ and $100 \%$ of the maximum g-force attainable by the vibrator. For each level of applied g-force, an array of frequencies from $0 \mathrm{~Hz}$ to $60 \mathrm{~Hz}$ was applied to the LSP in $10 \mathrm{~Hz}$ increments from low to high frequencies. For each frequency setpoint, data was collected for a sufficient amount of time as to accurately represent the resulting parameters. Each setpoint was conducted three times as to obtain an average value for that setpoint. No data points were removed regardless of deviation from the mean. 


\section{RESULTS AND DISCUSSION}

The following discussion pertains to the data gathered from the experimental investigation. The measured values and assumptions that are taken as constants in the calculated results are presented in Table 6 . These assumptions are consistent with previous experimentation conducted by Woodruff and McIntyre [29]. Additional graphical and numerical data that was collected in this experimentation can be found in Appendix-E and Appendix-F respectively.

Table 6: Assumptions and constants

\begin{tabular}{|c|c|c|c|c|}
\hline $\begin{array}{c}\text { Wavelength } \\
\mathbf{( n m})\end{array}$ & $\begin{array}{c}\text { Focal length } \\
\mathbf{( m m )}\end{array}$ & $\begin{array}{c}\text { Beam diameter at lens } \\
\mathbf{( m m )}\end{array}$ & $\begin{array}{c}\text { Beam quality } \\
\text { Focal area } \\
\mathbf{( c m} 2)\end{array}$ \\
\hline 1064 & 14.37 & 2.5 & 5.5 & $1.44 \times 10^{-5}$ \\
\hline
\end{tabular}

\subsection{Correction Factor}

During each output pulse from the LSP, a significant amount of $808 \mathrm{~nm}$ pump energy exits the laser cavity in addition to the $1064 \mathrm{~nm}$ output pulse. When the $808 \mathrm{~nm}$ pump energy enters the cavity is it is focused inside the Nd:YAG gain media. Not all of the $808 \mathrm{~nm}$ energy is absorbed by the gain media. The $808 \mathrm{~nm}$ energy that is not absorbed by the gain media passes through at a relatively high angle of divergence and exits the cavity.

Due to the proximity of the power meter's detector head to the output of the LSP, a correction factor for the output energy was necessary to account for transmitted $808 \mathrm{~nm}$ energy for the 45 degree and 90 degree mounting angles. The positioning of the detector head directly adjacent to the laser's output was necessary for these mounting angles due to spatial constraints of the experimental setup.

The correction factor data was collected with the optical axis of the LSP in a 0 degree orientation with respect to the horizon. Data was measured with the detector head the same distance from the laser output as it would have been if mounted on the oscillator $\left(d_{1}\right)$. In this position, both $808 \mathrm{~nm}$ and $1064 \mathrm{~nm}$ energy are measured by the detector. Data was also collected with the detector head $\sim 30$ inches back from the laser output $\left(d_{2}\right)$. This was deemed a suitable distance ,through experimentation, to collect only $1064 \mathrm{~nm}$ output by allowing the 808 
nm pump energy to significantly diverge. This is shown in Figure 29 where the red lines represent the $808 \mathrm{~nm}$ energy exiting the LSP at a high divergence angle and the green line represents the $1064 \mathrm{~nm}$ energy exiting the LSP as a collimated beam. By measuring the output energy with the detector head close to the laser's output and by measuring the output energy a reasonable distance away from the output, a ratio of $1064 \mathrm{~nm}$ energy to $808 \mathrm{~nm}$ plus $1064 \mathrm{~nm}$ energy was generated. This results in a percentage of $1064 \mathrm{~nm}$ energy from the total energy output as shown below in Equation 5-1.

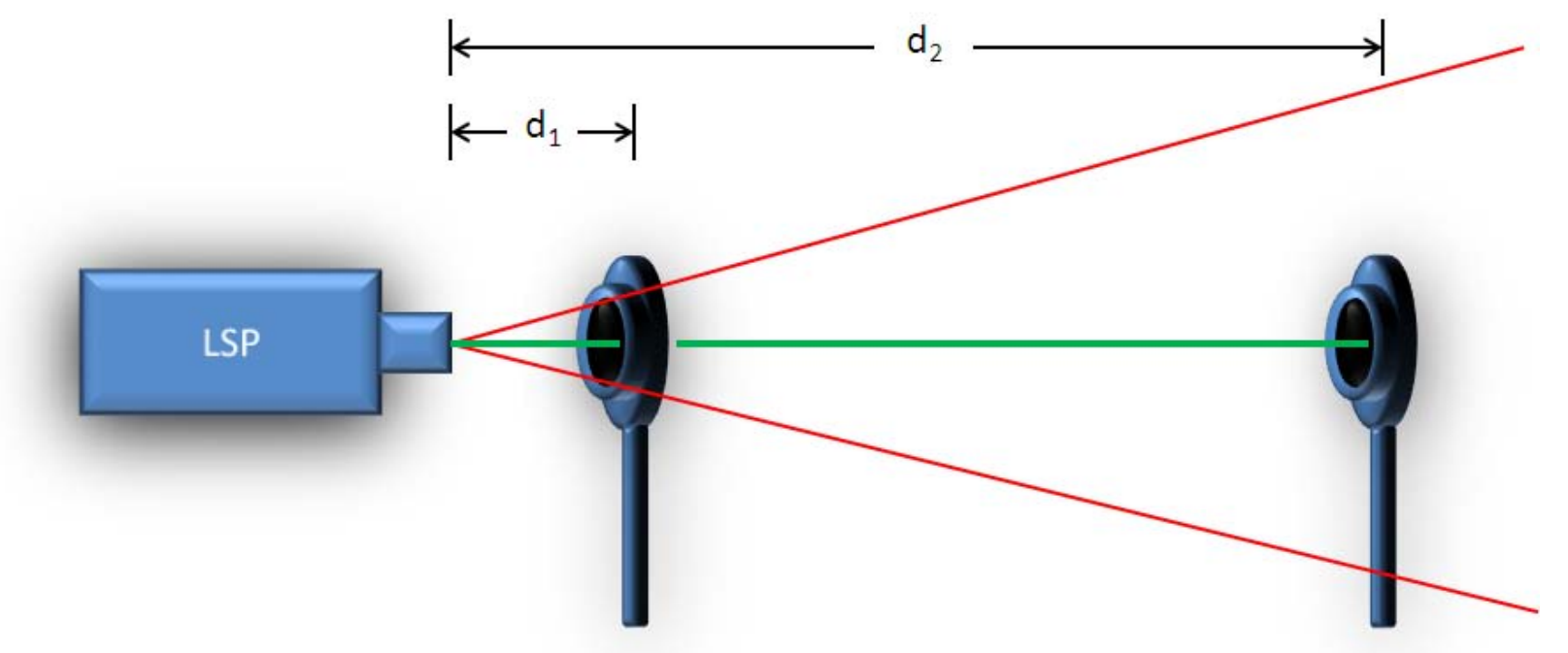

Figure 29: Detector head positioning for calculation of the correction factor

$\% E_{1064 n m}=\left(\frac{E_{1064 n m}}{E_{808 n m}+E_{1064 n m}}\right) * 100=\left(\frac{E_{2}}{E_{1}}\right) * 100$

The divergence of the pump beam and its effect on the measured energy is best described by the inverse square law, as shown in Equation 5-2, where the intensity of the beam is proportional to the inverse of the distance traveled squared where $I$ is intensity and $d$ is the distance or radius from the output. As the distance to the detector increases, the intensity of the $808 \mathrm{~nm}$ energy that the detector is capable of measuring is significantly reduced. Since intensity is power per area, and the area influenced by the diverging photons resembles the surface area of a sphere, the intensity scales inversely with the square distance the photons have traveled from 
the point source [56]. This method is sufficient because the $1064 \mathrm{~nm}$ energy exits the cavity with a nearly collimated profile and has very little divergence.

$I \propto \frac{1}{d^{2}}$

With the LSP wrapped in heat tape in a similar fashion as it would be if mounted on the oscillator, correction factor data was collected as a function of temperature and pump pulse frequency only. The literature review shows that the energy output for an end pumped passively q-switched Nd:YAG laser is a function of operating temperature and a function of energy absorbed by the media on a time average basis as well [34]. As vibrational frequency increases due to the increasing speed of an engine, the pump pulse frequency must increase respectively as well. The increase in pump pulse frequency yields a higher rate of energy absorbed by the Nd:YAG gain media. This increases the rate of thermal energy absorbed and affects the 1064 nm output energy as well.

The data shown in Figure 30 shows the correction factor data collected as a function of temperature. Each data point for a given temperature setpoint is an average of all of the data points for that temperature over the frequency range. For example, the 90 degree temperature data point in Figure 30 is the average of all the frequency data collected at that temperature. This was done to simplify the data presentation since the matrix conducted is in several dimensions. The $1064 \mathrm{~nm}$ output energy increases in a near linear fashion as a function of operating temperature. This is consistent with the findings by Bass et al. [34]. As temperature increases within the gain medium, the local energy increases as well making it easier for an atom to reach an excited state [24]. With a higher temperature, the input pump energy excites more atoms within the gain media yielding higher output pulse energy as previously discussed in Section 2.4.1.2. 


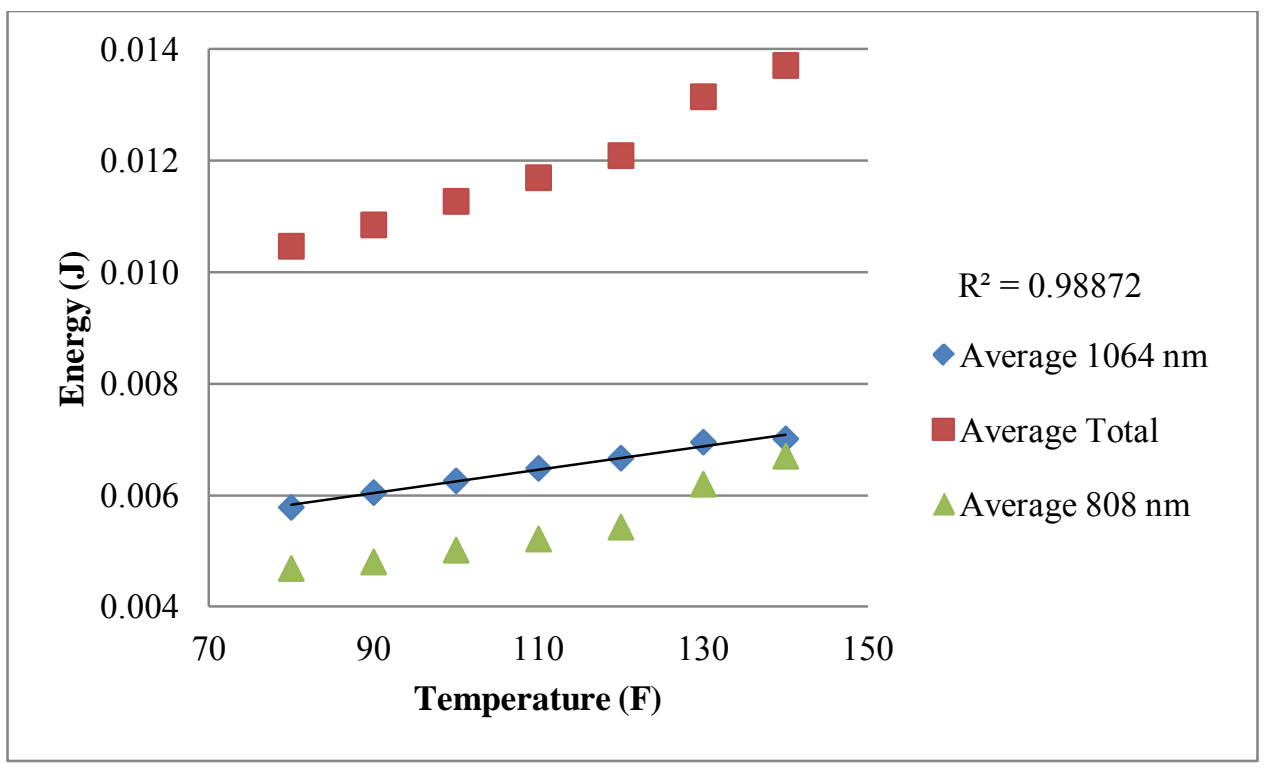

Figure 30: Average correction factor energy as a function of temperature

Also from an analysis of Figure 30, we see that the average $808 \mathrm{~nm}$ energy output increases with increasing temperature as well. As the temperature of the LSP is increased, it was found through preliminary investigations that the q-switch delay increases as a function of increasing temperature. This is accounted for by increasing the pump pulse width. Increasing the pump pulse width increases the total amount of $808 \mathrm{~nm}$ energy entering the ignition laser per pulse. Given the assumption that little q-switch absorption of the pump energy occurs by the saturable absorber in its bleached state, the $808 \mathrm{~nm}$ energy is able to pass through the q-switch and exit the LSP. This $808 \mathrm{~nm}$ energy is measured and corrected for in the 45 degree and 90 degree mounting orientations. The correction factor was not applied to the 0 degree mounting orientation because in this orientation, it was possible to locate the detector head in the "Far" position.

Upon examination of Figure 31 where the energy is shown as a function of pump pulse frequency, it can be seen that the average $1064 \mathrm{~nm}$ energy increases in a linear fashion with increasing pump pulse frequency. This is consistent with the literature [34]. The values presented here for a given pump pulse frequency is an average of all of the data points for that pulse frequency over the temperature range. For example, the $10 \mathrm{~Hz}$ pump pulse frequency data point in Figure 31 is the average of all the temperature data collected at that pulse frequency. With an increasing pump pulse frequency, the amount of thermal energy absorbed increases, 
causing the time integrated global temperature of the excitation mechanism to be higher. With a higher temperature, the input pump energy excites more neodymium atoms within the gain media yielding a higher ignition energy output pulse.

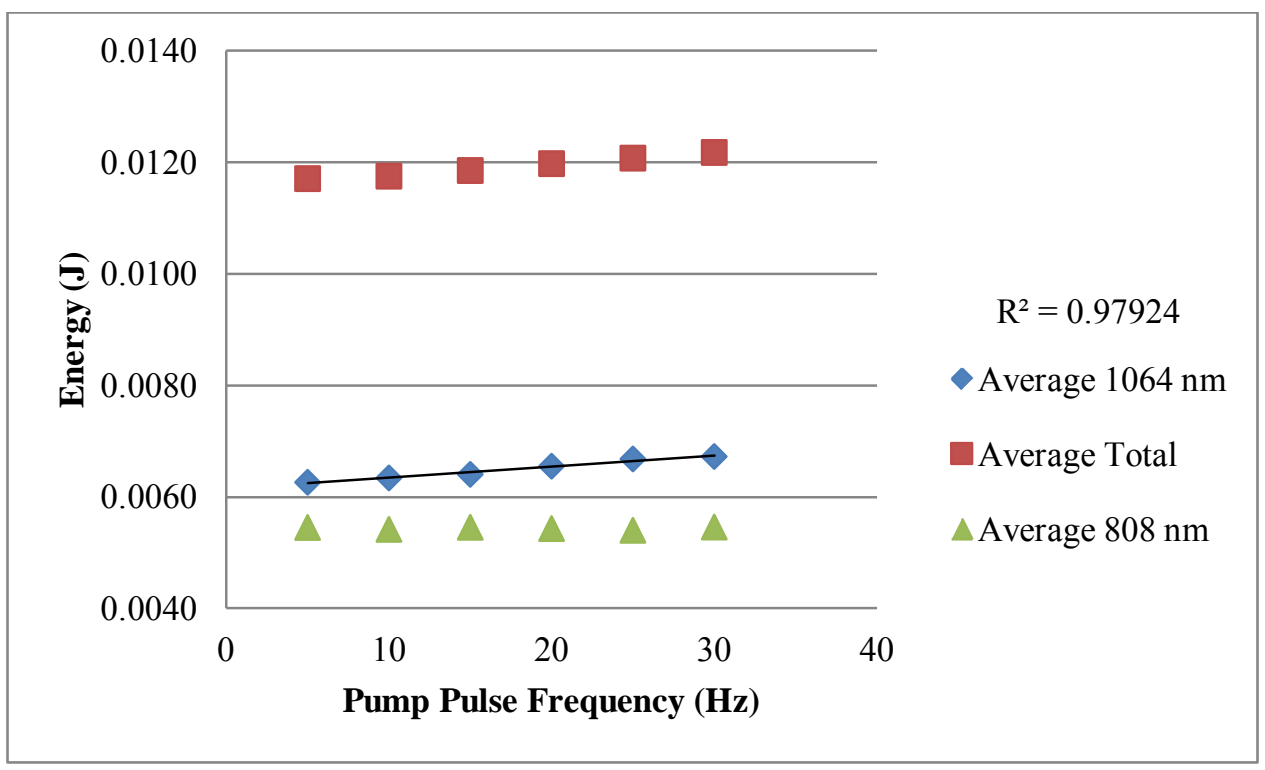

Figure 31: Average correction factor energy as a function of pump pulse frequency

Figure 31 illustrates that the average $808 \mathrm{~nm}$ energy output remains nearly constant, unlike in Figure 30 where the average $808 \mathrm{~nm}$ energy increases. Although the rate of thermal energy absorbed increases with increasing pump pulse frequency, the change in temperature is not significant enough to produce a noticeable change in the $808 \mathrm{~nm}$ pumping energy.

From an analysis of Figure 30 and Figure 31, it is evident that the average $808 \mathrm{~nm}$ energy exiting the cavity appears to be a strong function of heat tape temperature and a weak function of pump pulse frequency. Using this data, a correction factor accounting for the amount of $808 \mathrm{~nm}$ energy passing through the ignition laser's cavity was generated for each thermal and pump pulse frequency setpoint. The resulting percentages of $1064 \mathrm{~nm}$ energy to total energy as calculated from Equation 5-1 are graphically displayed below in Figure 32. 


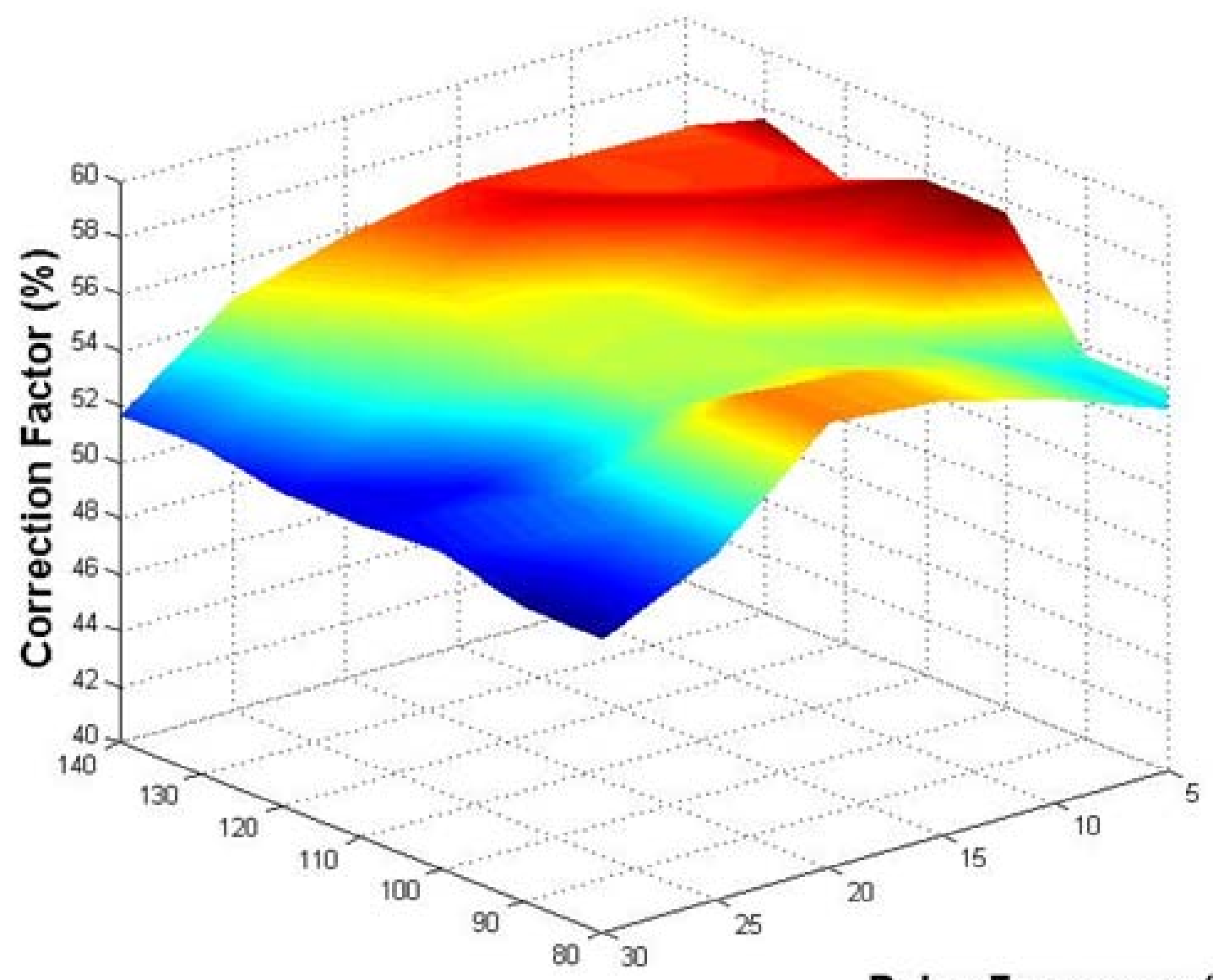

Temperature (F)

Pulse Frequency $(\mathrm{Hz})$

Figure 32: Applied correction factor percentage as a function of temperature and pump pulse frequency

The correction factor data points shown in Figure 32 represent the amount of $1064 \mathrm{~nm}$ energy present when the detector head is located near to the output of the LSP. As previously stated, the detector head was located near to the laser's output for the 45 degree and 90 degree mounting orientations. The array of correction factors were also applied to these data for each thermal and pump pulse frequency setpoint.

In an ideal passive q-switch, all of the chromium ions would be in their ground state. However this is not the case in real operation. Electrons may exist in slightly excited states due to temperature or inhomogeneous energy distributions. Excited state absorption occurs when an excited chromium ion interacts with $1064 \mathrm{~nm}$ laser energy, and is caused to go to a short lived higher excited state that does not contribute to the q-switch transmission. In this process, an 
atom in the saturable absorber remains transparent, but it absorbs the $1064 \mathrm{~nm}$ energy accounting for a loss. This loss process that can help explain the additional time needed for the q-switch to fire [34]. This causes a longer time for the saturable absorber to reach its bleached state yielding a longer q-switch delay as a function of increasing temperature. This can be seen in Figure 33 and Figure 34 where the q-switch delay has been found to increase with pump pulse frequency as well as with heat tape temperature. The margins of error presented here are one standard deviation from the mean of the data. They are large because they are averaged over the pump pulse frequency band (Figure 33) and heat tape temperature band (Figure 34).

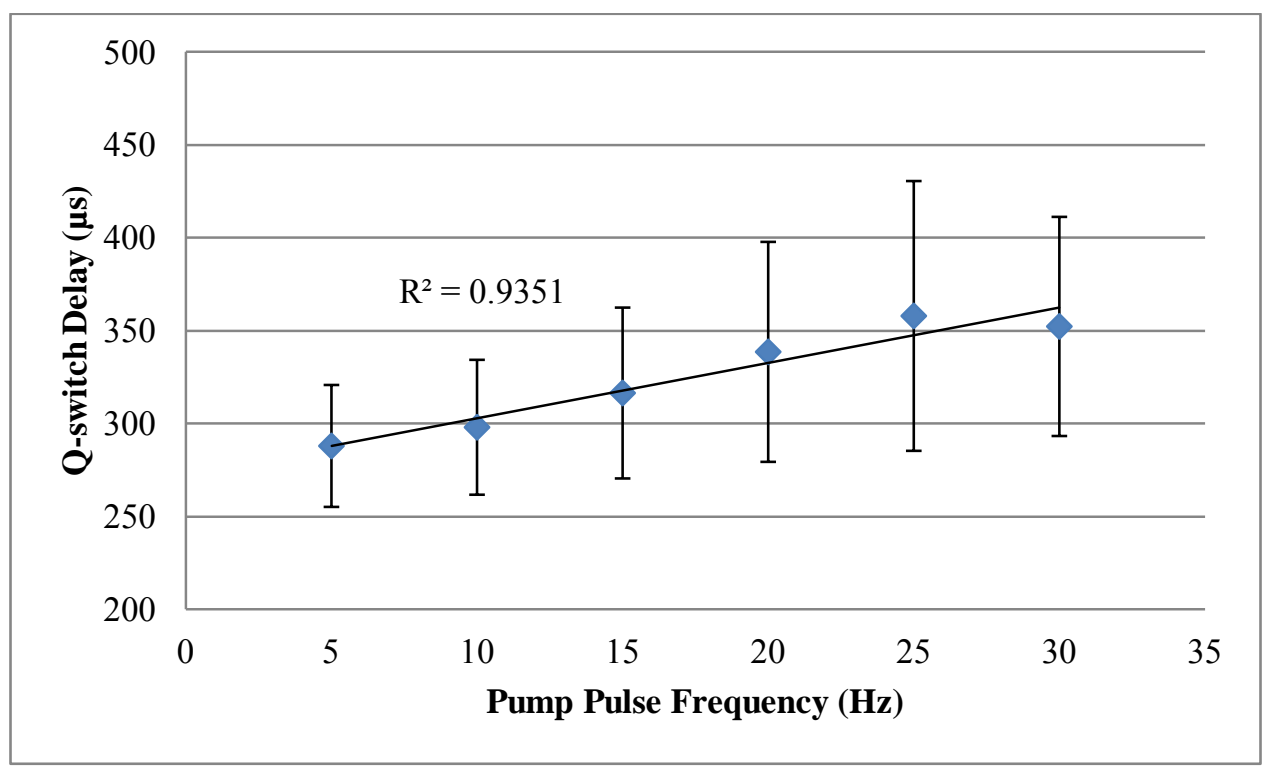

Figure 33: Average q-switch delay as a function of pump pulse frequency in correction factor orientation 


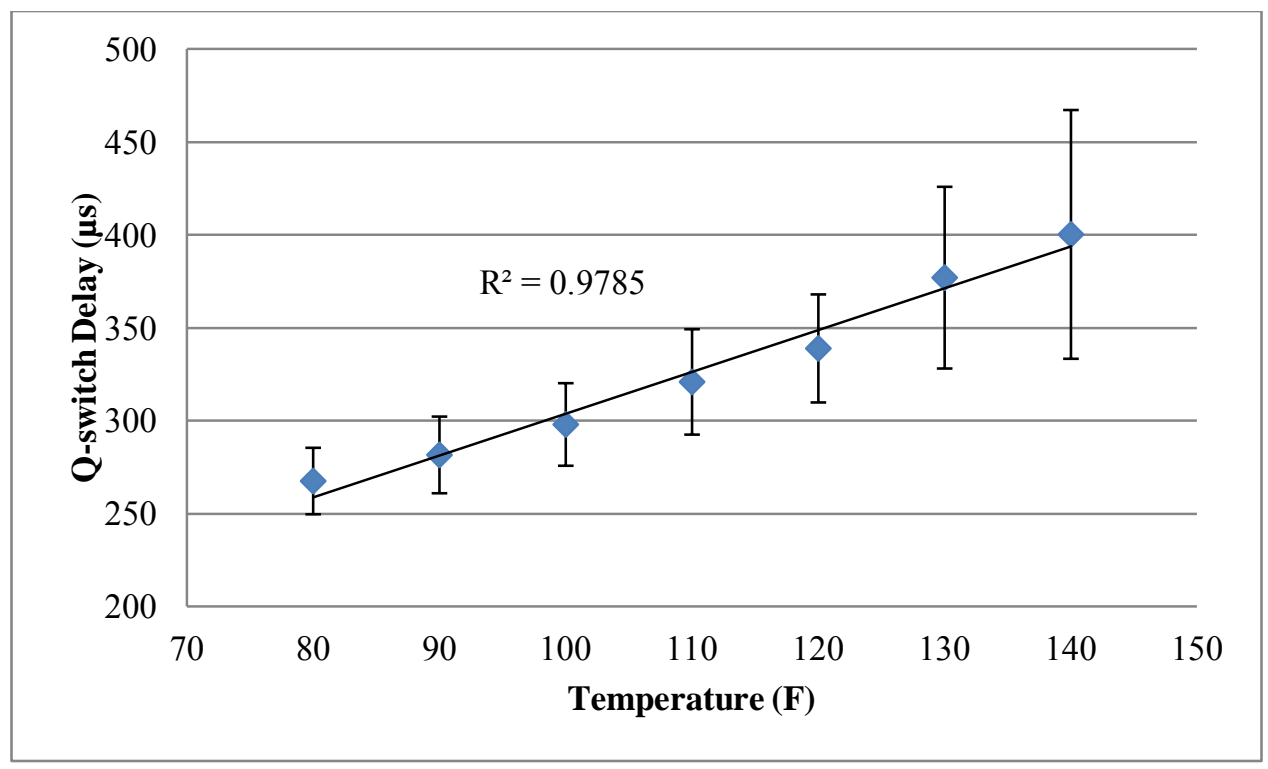

Figure 34: Average q-switch delay as a function of heat tape temperature in correction factor orientation

With a longer q-switch delay, there is more time for the pump source to generate a large population inversion within the gain media. This effectively causes a higher build up of photons in the laser's cavity. As shown in Figure 35, as temperature increases, the q-switch delay increases in a linear fashion. This effectively yields an increased energy output as a function of increased temperature. This is mathematically described in Equation 5-3 where $E$ is the output energy, $T$ is temperature and $\sigma$ is the atomic cross section [34].

$\frac{d E}{d T}=E\left(-\frac{1}{\sigma} \frac{d \sigma}{d T}\right)$

Equation 5-3 represents the change in output energy as a function of changing temperature and atomic cross section. It describes the roll temperature plays in the output energy of an ideal Cr:YAG passively q-switched Nd:YAG laser. In the case of a real q-switch, there are some ions that remain in the ground state during bleaching which vary the actual operation of the laser. Equation 5-3 holds true based on the assumption that there are no ions left in their ground state during bleaching. 


\subsection{Temperature}

The influence of temperature was found to have a considerable impact on the characteristics of the output pulse from the LSP. Pulse width, pulse width variation, q-switch delay, jitter and energy output were all found to vary with respect to temperature. The variation of these parameters can potentially lead to cycle-to-cycle variation within the combustion chamber through mechanisms such as ignition delay, ignition timing variation and ignition efficiency. The following sections discuss the temperature dependence of the LSP and the dependent variables collected from the oscillatory and thermal testing.

The use of bulk crystals in high power laser systems continues to be the best solution for achieving high peak power outputs. Although these laser crystals are convenient they are fundamentally limited by thermal loading that causes thermo-optical and thermo-mechanical perturbations to present themselves $[32,57,58]$. This yields an undesirable parameter referred to as thermal lensing. Thermal lensing is the increased refraction angle of light waves from a reflective surface when the surface is subjected to nonsymmetrical thermal loading. It was proved to be a major source for power fluctuations and an inconsistent $\mathrm{M}^{2}$ allowing the laser operation to deviate from its optimized condition $[32,57,58]$. The thermal cycling and thermal conductivity of the crystal produces inconsistent thermal gradients that can affect operation and overall performance of the laser. These variable thermal gradients are caused by heterogeneous pump source distribution, inconsistencies of the beam trajectory in the rod and heterogeneous distribution of the doping concentration.

Heat repartition and thermal conductivity directly influence the thermal profile of the crystal and ultimately impose optical index gradients. These gradients affect the wave front propagation of the beam leading to unwanted variation in the system. Thermal lensing causes variation from axial parallel beam transmission within the laser cavity which strongly affects the characteristics of the output beam [32]. When considering the thermal effects from pumping, energy is absorbed in the gain media due to non-radiative transitions, impurities and inhomogeneities. In Nd:YAG for example, $30 \%$ of the pumping energy is lost due to the thermal absorption of the rod [32].

Thermal expansion of the materials comprising the structure of the LSP was found to have a significant effect on the alignment. The thermal expansion of the steel used for the 
mounts is $8.8 \mu \mathrm{in} /{ }^{\mathrm{F}} \mathrm{F}$. When incorporating the temperature difference between $80^{\circ} \mathrm{F}$ and $140^{\circ} \mathrm{F}$, it was calculated the LSP alignment may have shifted by over 500 $\mu$ in. The need for pinpoint accuracy is critical in order to obtain efficient, reliable and consistent output characteristics.

\subsubsection{Pulse Width}

The oscilloscope used to measure the pulse width has a built in function which measures the pulse width and reports the resulting measurement numerically in $5 \mathrm{~Hz}$ sampling intervals on the screen as shown in Figure 35. Temperature and vibration were predicted to not only have an impact on the pulse width of the beam but also on the variation of the pulse width. At each setpoint, the numerical display was monitored and the minimum and maximum readings were recorded. The pulse width was defined as the average between the minimum and maximum reading and the pulse width variation was defined as difference between the minimum and maximum readings divided by two. An attempt was made to capture this data through the DAQ system, however limitations of the equipment prohibited this. This presents an addition of human error into the tabulated results which is not quantified.

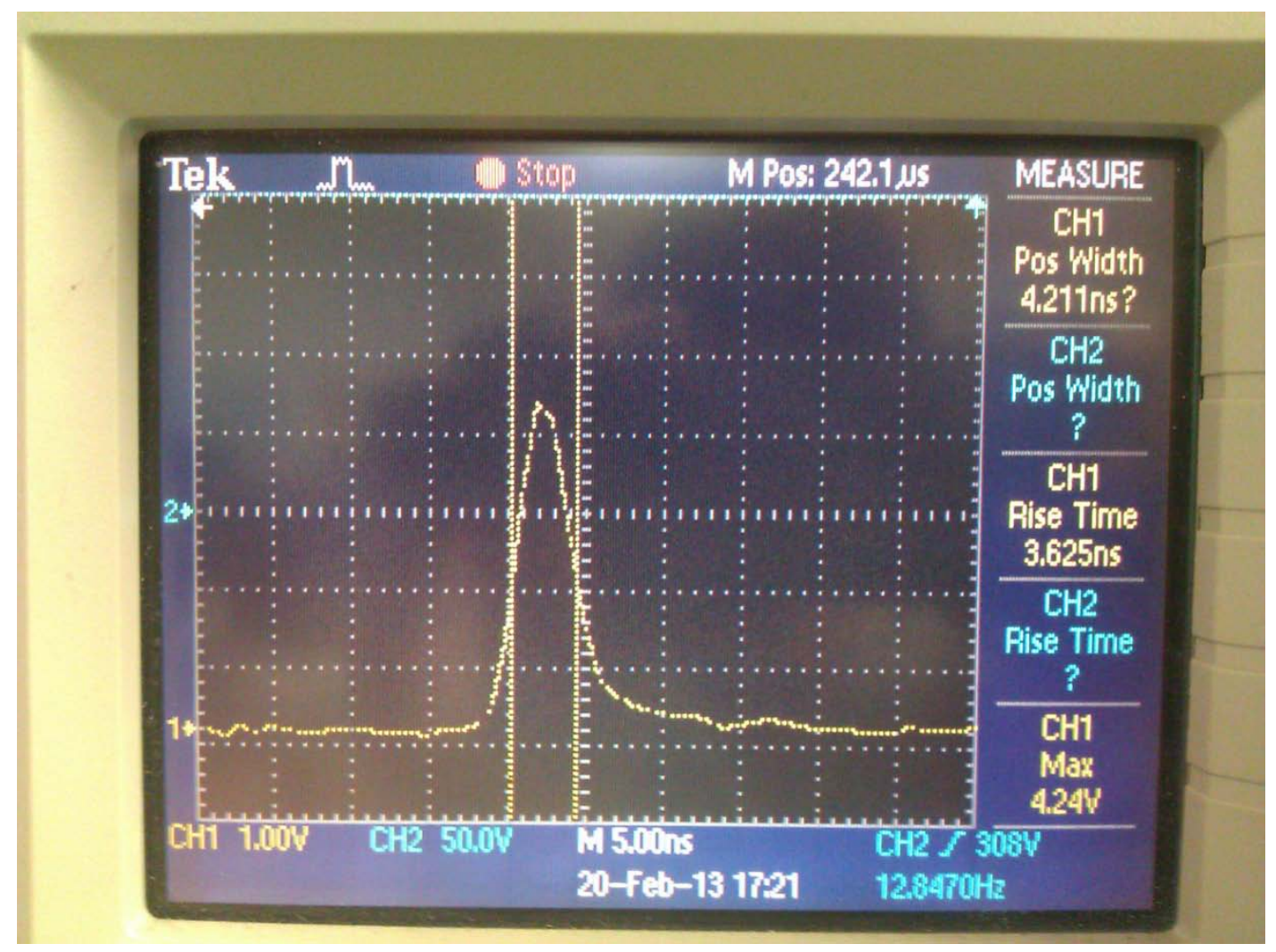

Figure 35: Pulse width measurement using the oscilloscope 
The width of the output pulse has a significant impact on the combustion efficiency and combustion stability in IC engines. A pulse that is too short may yield a high intensity plasma spark, but may lack sufficient duration to effectively initiate the combustion process. Too wide of a pulse width may decrease the focal intensity to a point that is below the breakdown threshold of the gas. Therefore an optimum pulse width exists for LI applications. This relationship between focal intensity and pulse width is shown below in Equation 5-4, where $I$ is the focal intensity, $E_{\text {out }}$ is the output energy, $t_{p}$ is the FWHM output pulse width and $A$ is the focal spot size area. The focal spot area in fundamentally limited by the operating wavelength, $\mathrm{M}^{2}$ and focal length of the optic as well. For ignition applications, there exists an optimal pulse width range that is short enough to yield a high focal intensity but long enough to effectively allow for enough energy to be transferred to the medium to initiate combustion. At room temperature, pulse width remains approximately constant because it is a strong function of cavity length.

$I=\frac{E_{\text {out }}}{t_{p} A}$

Figure 36 shows the trend of the pulse width measurements as a function of temperature. The average pulse width has been found to vary by as much as \pm 0.2 ns across the temperature setpoint band. There is strong potential for this variation to be due to the error in the instrumentation. Although a variation exists in the collected pulse width data, the actual change in pulse width appears to be nearly independent of mounting orientation or temperature. 


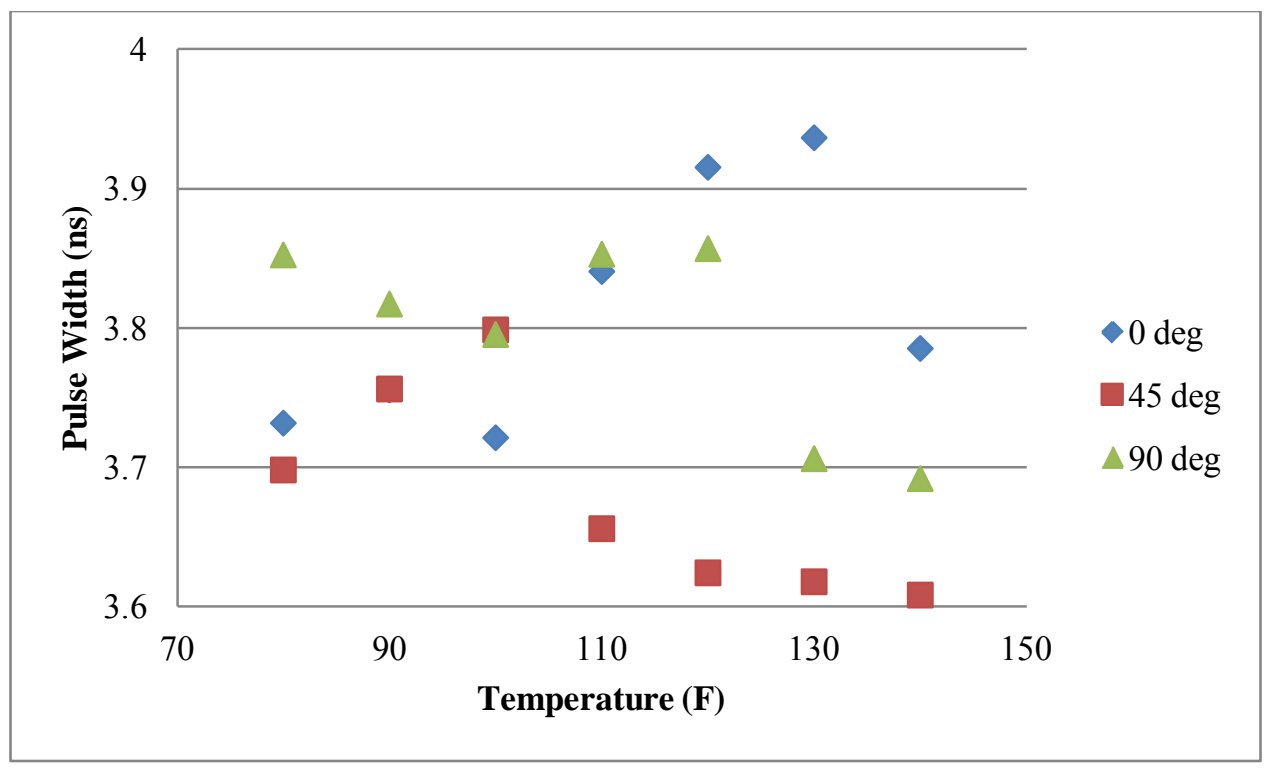

Figure 36: Pulse width as a function of temperature for 0,45 and 90 degree mounting orientations with $100 \%$ acceleration

Figure 37 shows the pulse width as a function of temperature for the 90 degree orientation at $100 \%$ acceleration with margins of error encountered with the measurement collection. The bars represent one standard deviation from the mean combined with the pulse width variation for each setpoint.

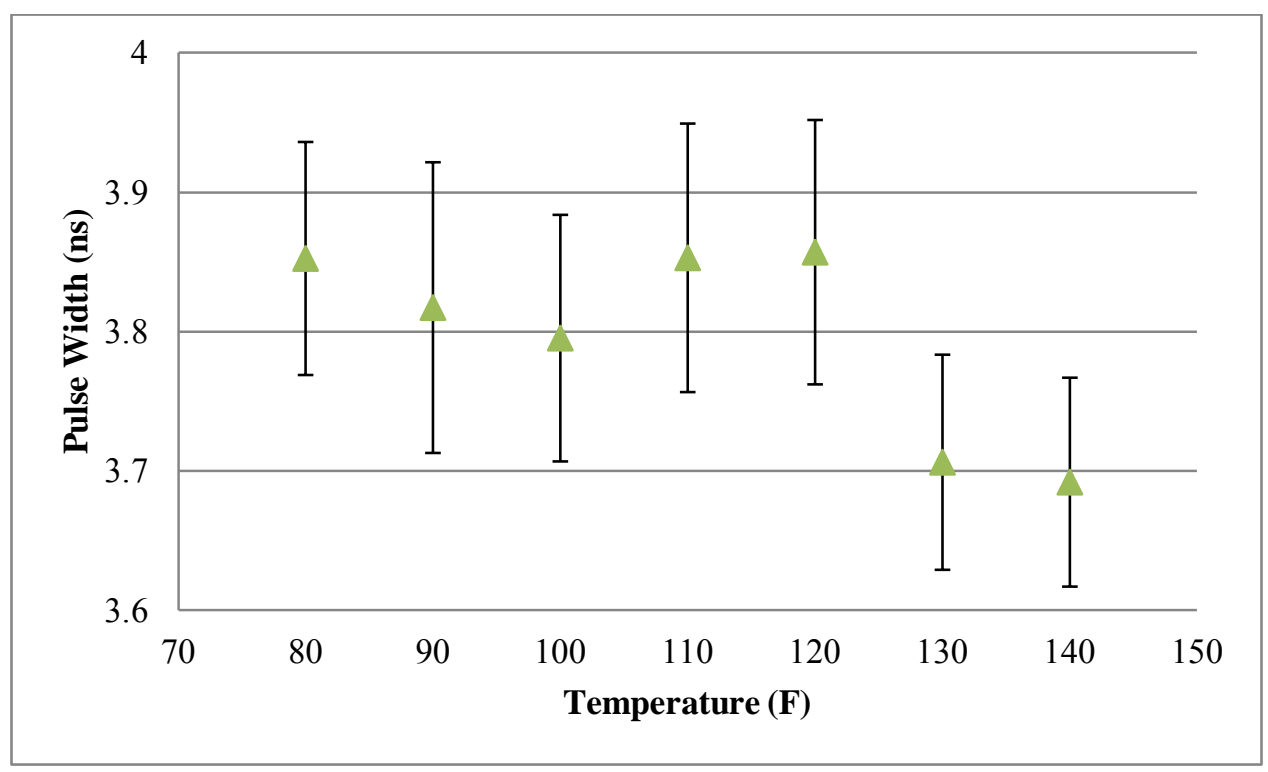

Figure 37: Pulse width as a function of temperature for the 90 degree orientation with 100\% acceleration 
Since the average pulse width has been found to remain consistent with respect to temperature and mounting angle with significant cycle-to-cycle variations, a means of minimizing this variation in the pulse width is necessary to ensure consistent ignition parameters. Fueling and ignition strategy must be controlled based on the operating pulse width of the LSP. Since pulse width strongly affects the focal intensity, fixing the pulse width and minimizing its variation is desirable.

The ignition pulse width is a function of the length of the cavity and the switching rate of the saturable absorber. The decay rate of a particular saturable absorber is largely dependent upon its material properties and the pumping strategy at which it is being operated. The length of the cavity has the potential to optimize the pulse width for a given application based on the engine's control parameters and flow geometry of the engine. Minimization of the pulse width variation in such a system is the key to obtaining consistency.

\subsubsection{Pulse Width Variation}

Pulse width variation has the potential to cause cycle-to-cycle variation within the system. With increasing temperature, the magnitude of the variation in the pulse width was found to fluctuate for each mounting angle. Although the results show variation on the tens of picoseconds scale, there exists room for improvement. Figure 38 shows how the variation in the pulse width trends under the influence of increasing temperature for each mounting orientation at the $50 \%$ acceleration setpoints. 


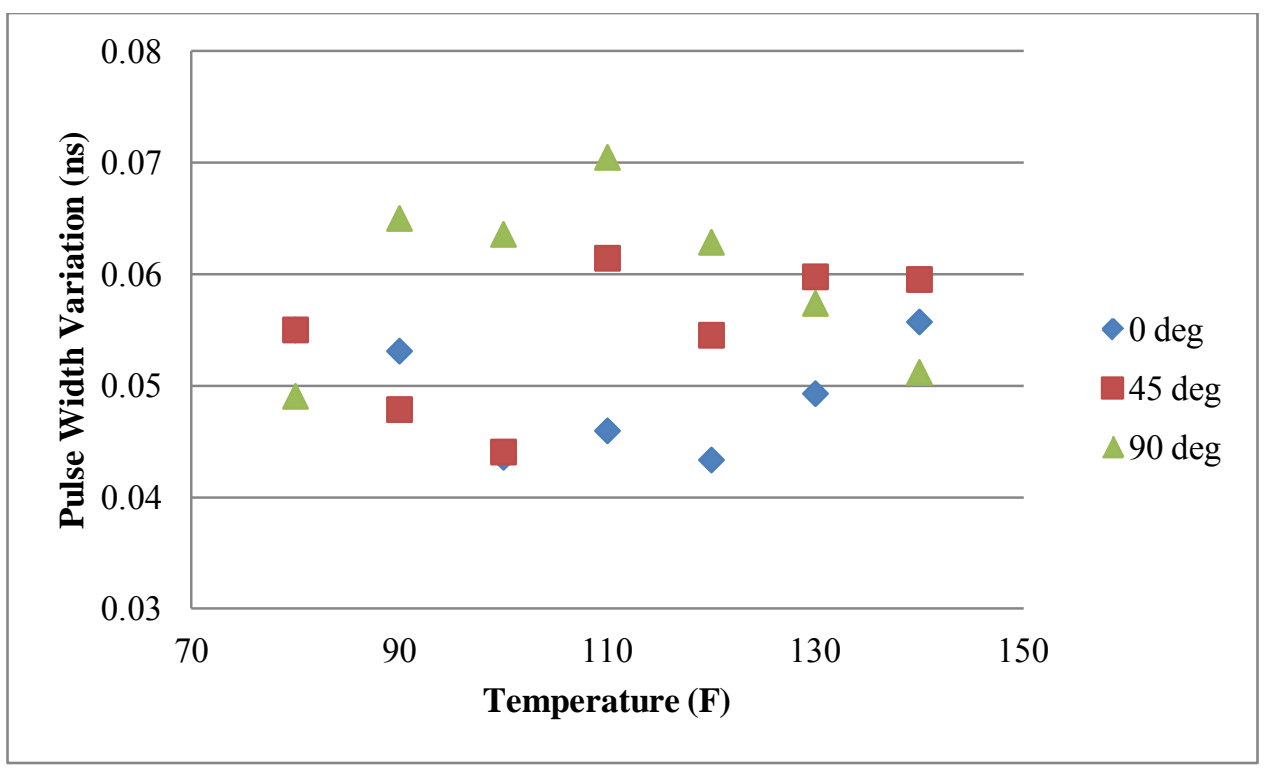

Figure 38: Pulse width variation as a function of temperature for 0,45 and 90 degree mounting orientations with $50 \%$ acceleration

In an ideal case for applying for LI system of IC engine applications, the variation in the pulse width would be zero. However this is not the case in a real system. There will always be a certain degree of variation based on the heterogeneity of the system. An average pulse width may be computed for a particular operating temperature and orientation, however there is no means of predicting the exact pulse width variation for a given ignition event. This presents a variability parameter that cannot be compensated for by ignition or injection timing variation. Therefore, a means of minimizing the variation in the pulse width is necessary for ignition optimization which is further discussed in Section 6.2. Figure 39 shows the variability in the pulse width variation for the 45 degree mounting orientation at 50\% acceleration. The bars presented here are one standard deviation away from the pulse width variation mean for each thermal setpoint. 


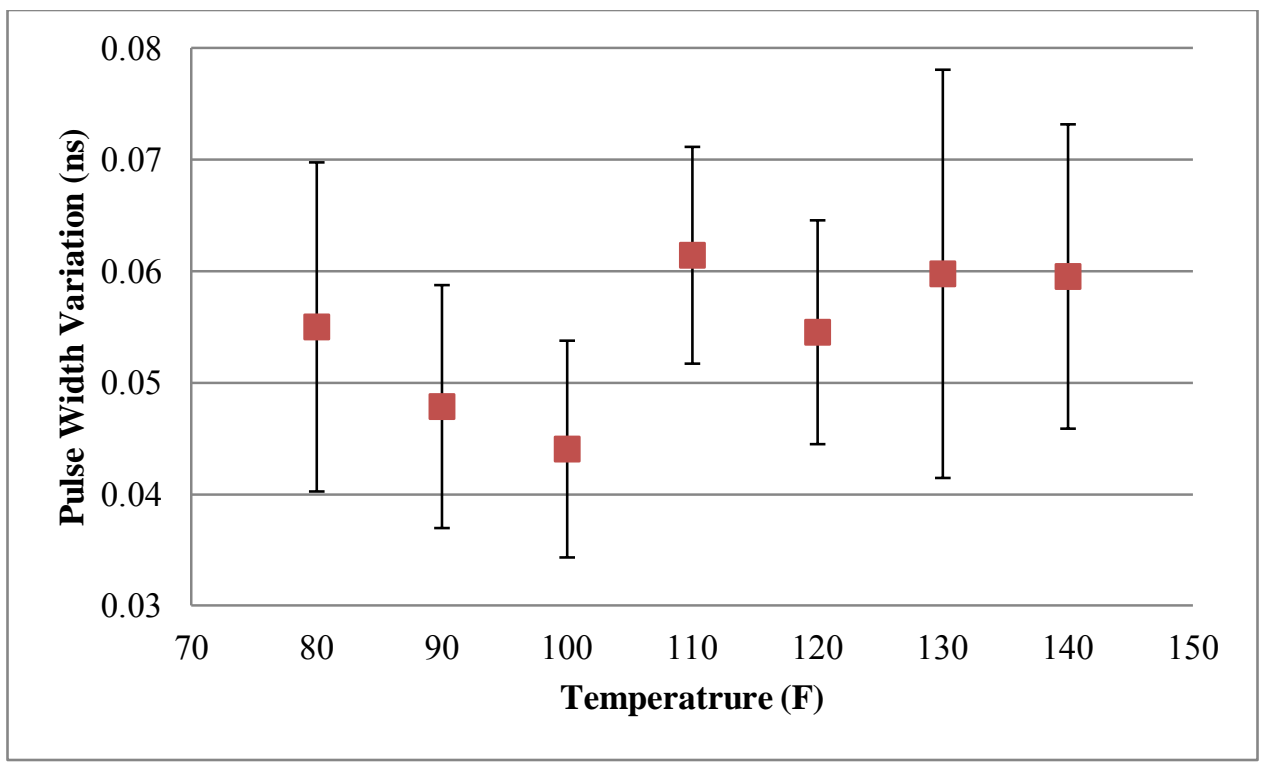

Figure 39: Pulse width variation as a function of temperature for the 45 degree mounting orientation with $50 \%$ acceleration

The margin of error in the pulse width variation accounts for nearly $50 \%$ of the variation in the pulse width. This large magnitude of variation was attributed primarily to the laser chassis. As the payload was heated and oscillated in each respective orientation, flexing of the mounts and thermal expansion of dissimilar materials leads to minute variations in cavity path length and the alignment on a per cycle basis. In order to minimize the amount of variation in the pulse width, a chassis must be developed that is resistant to vibration and thermal influences. The smaller and more compact the payload given the same durability as the present system, the less susceptible to vibrational and acceleration influences the laser will be. The closer the intracavity optical components are to each other, the smaller the effects of temperature will have on the laser's output. Pulse width variation had a significant room for improvement in this design.

\subsubsection{Q-switch Delay}

The delay of the q-switch is an important parameter which significantly affects the ignition timing of the engine. Precision control over ignition timing is imperative for maximum combustion stability, power output and resulting emissions. As previously defined, the delay 
presented here is defined as the time from when the pump laser is triggered until the saturable absorber bleaches producing a pulsed $1064 \mathrm{~nm}$ output as previously shown in Figure 5.

Q-switch delay was found to increase with increasing temperature as shown in Figure 40 for the 0 and 90 degree orientations. An increase in the q-switch delay effectively yields an increase in the ignition delay. The resulting q-switch delay for the 0 and 90 degree mounting orientations was found to increase by as much as $130 \mu$ s and $111 \mu \mathrm{s}$, respectively. When considering this system to be operating on an engine from cold start to standard operating temperature, this change in q-switch delay may cause the point of ignition to change by as much as 4.6 CA degrees.

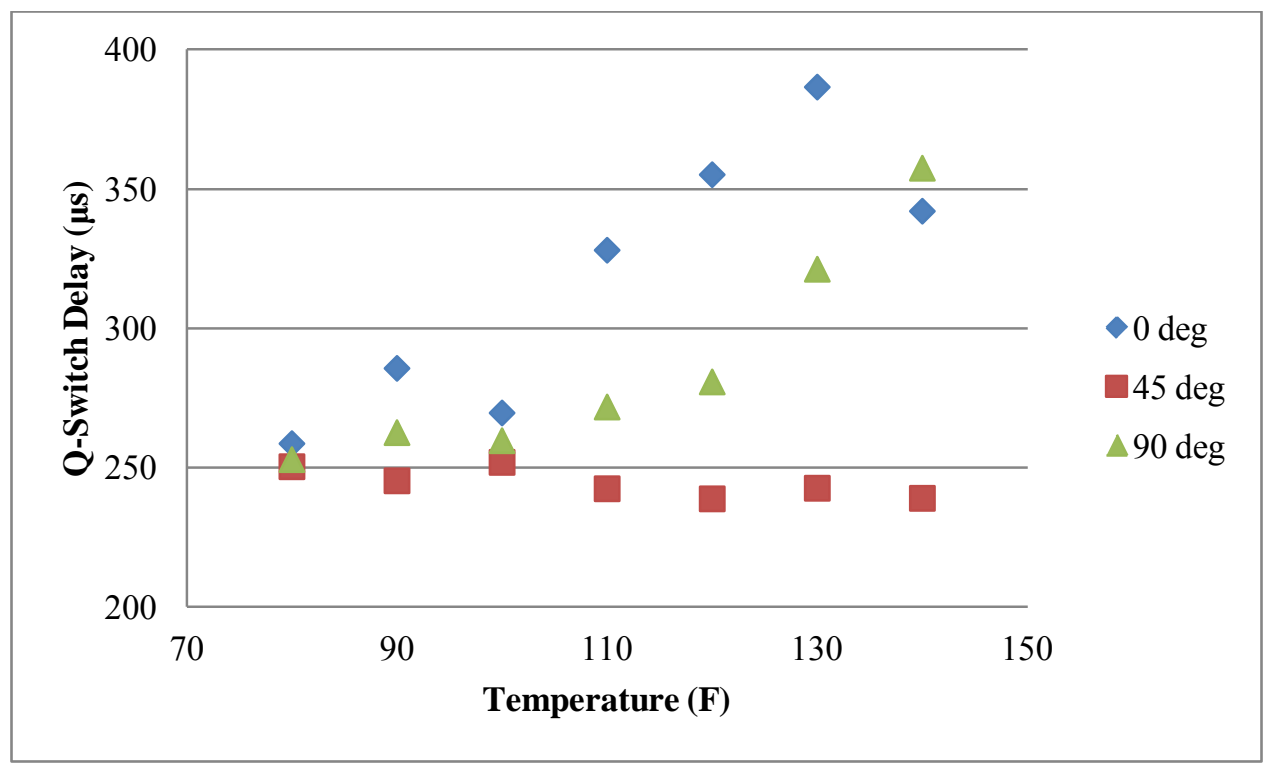

Figure 40: Average q-switch delay as a function of temperature for the 0,45 and 90 degree mounting orientations with $100 \%$ acceleration

Also shown in Figure 40, the average q-switch delay appears to remain constant across the thermal setpoints for the 45 degree mounting orientation. The 0 degree and 90 degree orientations were tested first and the 45 degree orientation was the final mounting orientation to be tested. It is a possibility that the 45 degree angle is the most ideal orientation for the LSP. However, it is also possible that a near perfect alignment was obtained for the 45 degree mounting orientation. It should be noted that for the 0 degree, 90 degree and correction factor orientations, several alignments were performed for each thermal setpoint, and in some cases multiple alignment procedures were performed during a single thermal setpoint. In the case of 
the 45 degree mounting orientation, a single alignment and optimization procedure was initially conducted at $80^{\circ} \mathrm{F}$ and a second alignment and optimization procedure was conducted at $90{ }^{\circ} \mathrm{F}$. Other than these two alignment procedures, no other alignments were performed for this mounting orientation. As shown in Figure 41, the variation of the q-switch delay in the 45 degree mounting orientation is minimal. Even with the bars in place, which represent one standard deviation from the q-switch mean in addition to the jitter, it is shown that the delay remains fairly constant throughout the thermal setpoints for the 45 degree mounting orientation.

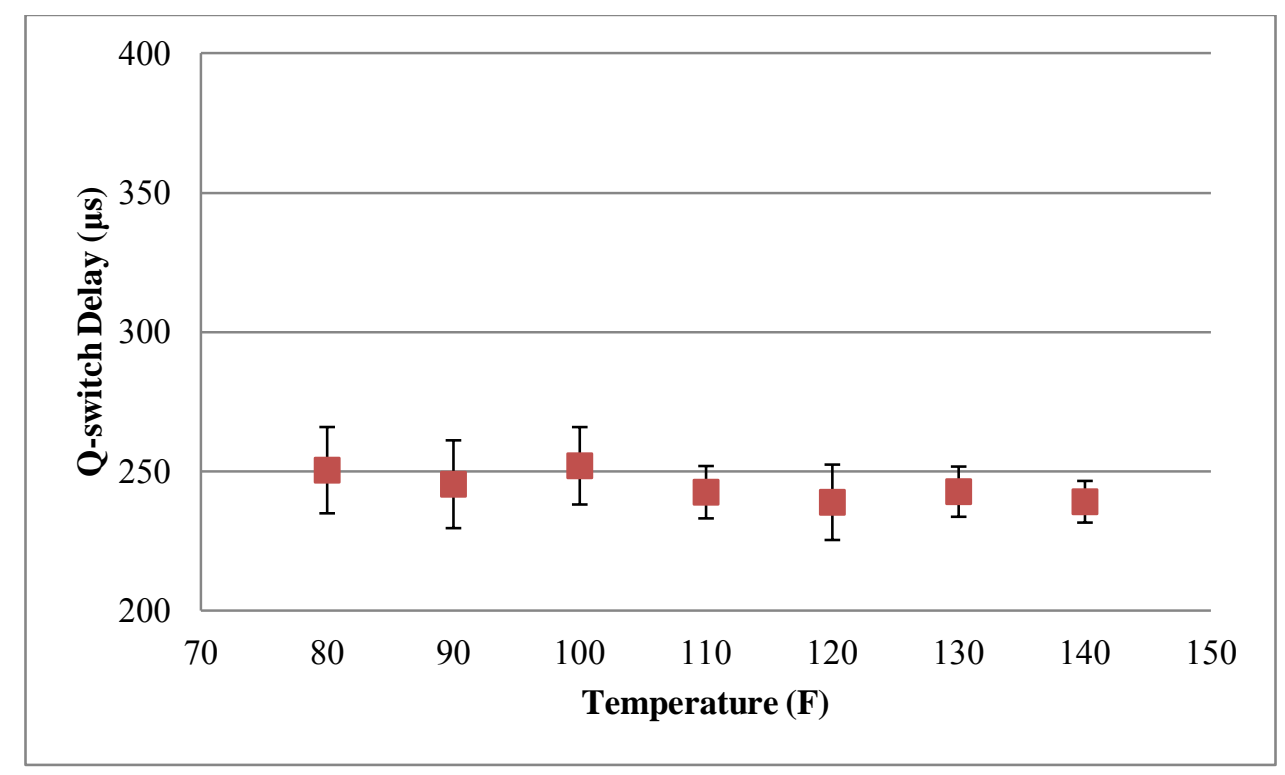

Figure 41: Average q-switch delay as a function of temperature for the 45 degree mounting orientation with $100 \%$ acceleration

Alignment variation is the most critical factor pertaining to the q-switch delay of a passively q-switched laser. As temperature increases, thermal expansion of dissimilar materials causes stress which leads to unwanted compression and tension in the LSP's chassis. This causes a shift in the alignment as well as modification of the pump lasers coupling efficiency with the gain medium. When the $808 \mathrm{~nm}$ pump energy is ineffectively coupled to the gain medium, a smaller rate of energy absorption occurs in the q-switch yielding a longer time for the saturable absorber to reach its bleaching threshold. This can be graphically seen in Figure 42 for the 90 degree mounting orientation where the increase in temperature causes an increase in qswitch delay. Also as temperature increases, the variation in the q-switch delay increases as well 
as shown by the bars increasing in size as temperature increases. This is consistent with the alignment of the optics being more variable at higher temperatures.

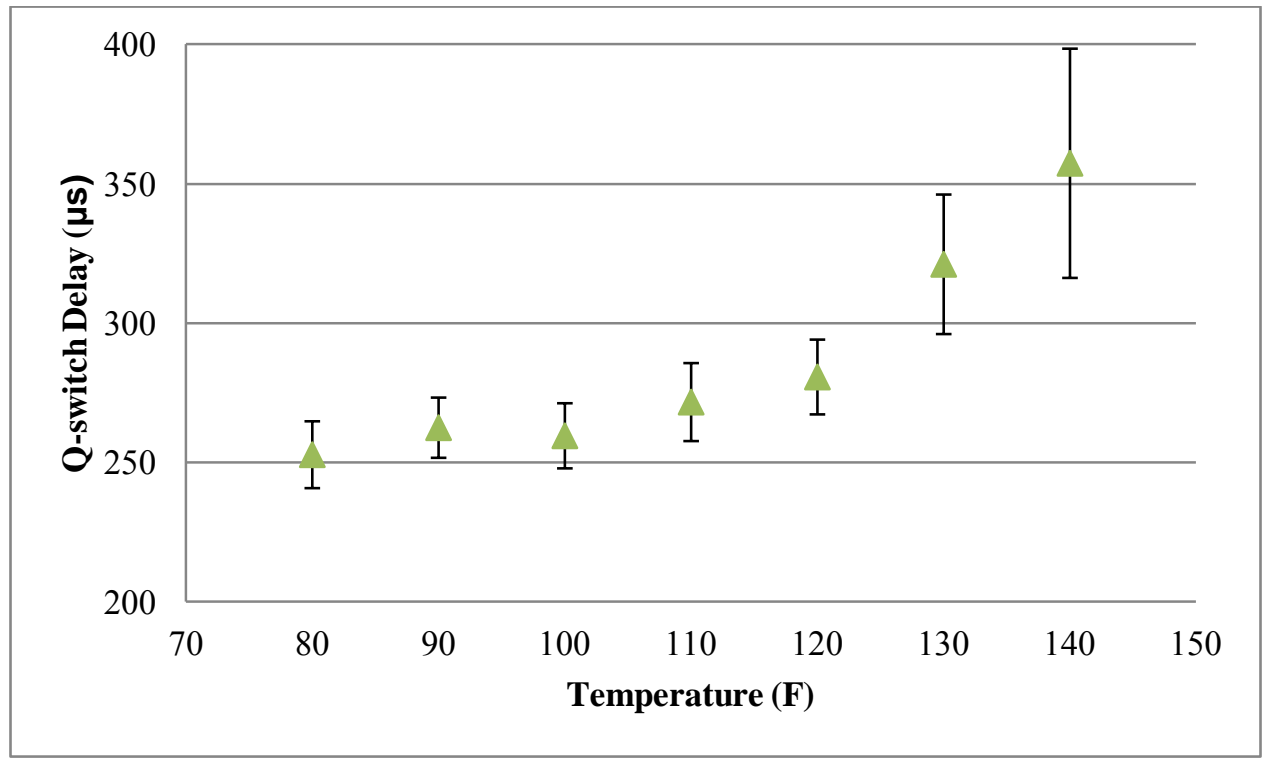

Figure 42: Average q-switch delay as a function of temperature for the 90 degree mounting orientation with $100 \%$ acceleration

As temperature is increased, the thermal expansion of the materials in the laser's chassis causes a variation in the optical alignment of the LSP. This results in a longer time to generate a spark since the alignment and coupling efficiency decreases as a function of increasing temperature. This presents an issue since the engine operates at increasingly higher temperatures as it goes from cold start up to normal operating temperature.

An interesting result that should be mentioned is the measured q-switch delay presented here when compared to the q-switch delay measured in previous experimentation by McIntyre et al. In Figure 43 where the q-switch delay is shown as a function q-switch initial transmission percentage, it can be seen that the resulting q-switch delay at $10 \%$ initial transmission is roughly twice the measured q-switch delay presented here. An explanation for this would be that a better alignment was obtained in this investigation thereby increasing the pump's coupling efficiency. 


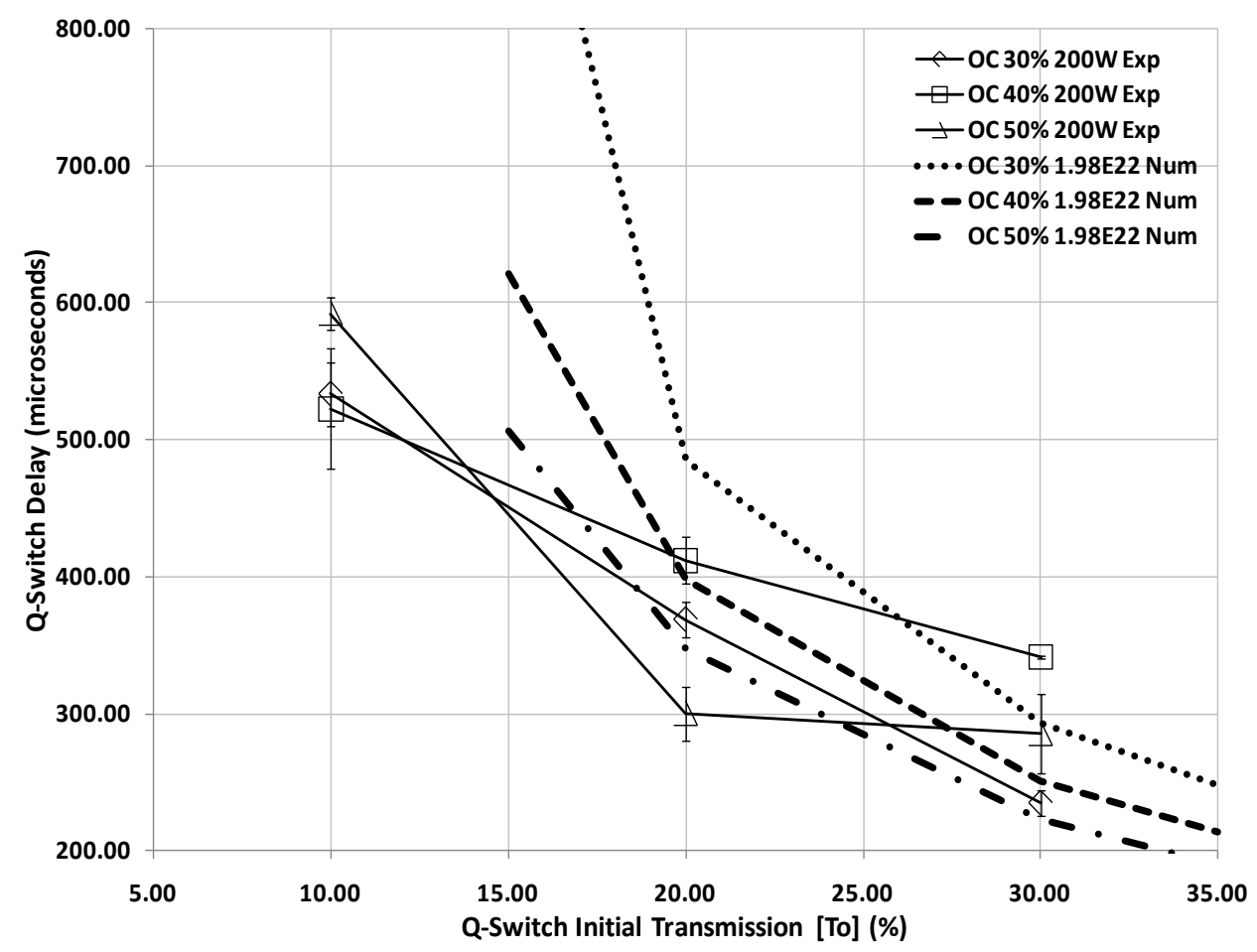

Figure 43: Q-switch delay as a function of q-switch initial transmission [29]

\subsubsection{Jitter}

Jitter is an important operating parameter of a LI system, which affects the ignition delay as well as the ignition timing. For the purposes of this investigation, jitter is defined as the deviation of q-switch delay from the average q-switch delay. For a LI system such as this, significant jitter is highly undesirable. The more jitter that is present in this optical system the more diverse the cycle-to-cycle variation will be. Jitter may lead to combustion initiation instabilities as well as a decrease in the time averaged combustion efficiency. As shown in Figure 44, jitter was found to increase with increasing temperature for the 90 degree mounting orientation. A maximum jitter of $27 \mu$ s was found to occur at the highest thermal setpoint with $100 \%$ acceleration. Assuming that the engine is operating at $3600 \mathrm{rpm}$, this yields a variation of $\pm 0.6 \mathrm{CA}$ on a per cycle basis simply due to the thermal effects on the laser's operation. 


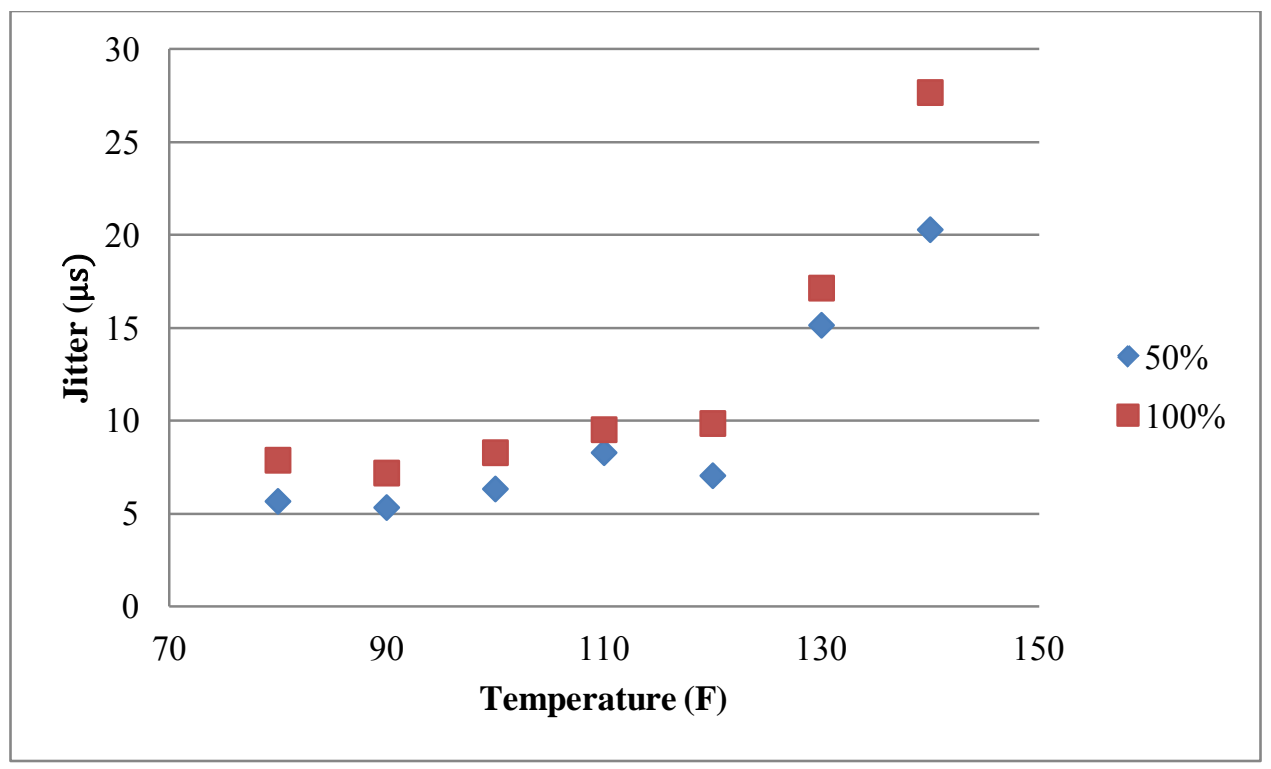

Figure 44: Average jitter as a function of temperature for $50 \%$ and $100 \%$ acceleration for the 90 degree mounting orientation

The variation in the q-switch delay that was observed on the oscilloscope appeared as a gradual transition in delay time, whereas the jitter appeared as a shot-to-shot variation superimposed upon the temporally varying q-switch delay. The jitter and q-switch delay that was observed on the oscilloscope is shown in Figure 45, where $Q_{d}$ is the q-switch delay and $\delta$ is the jitter. This reciprocation in the q-switch delay did not appear to follow any particular pattern and was unpredictable. As temperature increased, the magnitude at which the delay deviated increased for the 90 degree mounting orientation and the 0 degree mounting orientation as well. Jitter appeared in the system in a seemingly random and sporadic fashion. Regardless of the setpoint, jitter was always measured to be at least an order of magnitude less than the q-switch delay. 


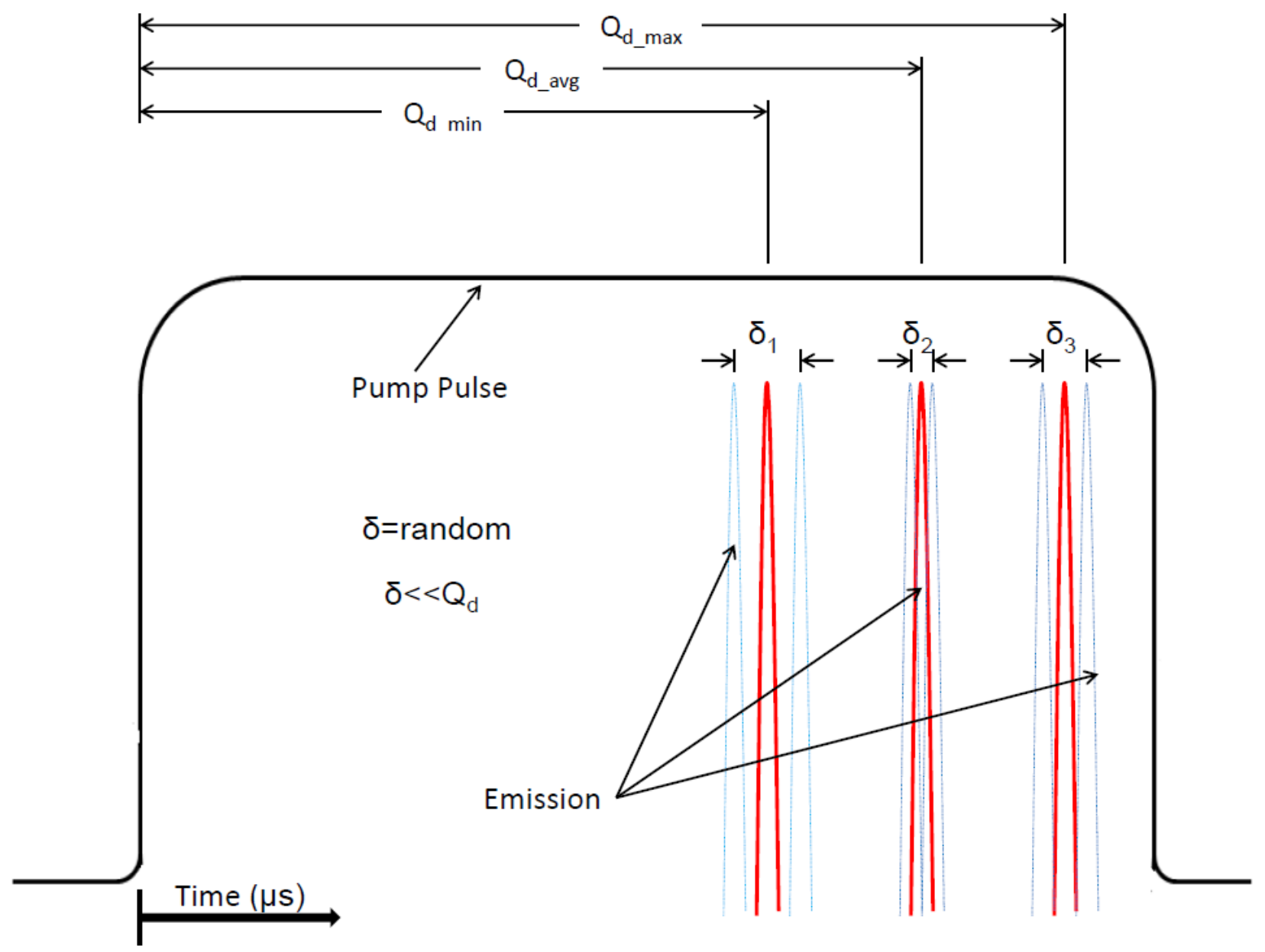

Figure 45: Variation in the q-switch delay and the jitter as a function of time as observed on the oscilloscope

At low temperatures the difference between $Q_{d_{-} \min }$ and $Q_{d_{-} \max }$ was low yielding a small standard deviation, while as temperature increased the difference between $Q_{d_{-} \min }$ and $Q_{d_{-} \max }$ typically increased resulting in a higher standard deviation at higher temperatures. This can be seen by the bars in the q-switch delay as previously shown in Figure 42. At lower temperatures the variation in $\delta$ was small, and at higher temperatures the variation in $\delta$ was high as shown in Figure 44.

Figure 46 shows average jitter results as a function of frequency and temperature for the 90 degree mounting orientation at $100 \%$ acceleration. From the magnitudes presented here, it can be seen that with the combination of thermal and oscillatory effects that the jitter is significantly higher than it would be if the induced perturbations were purely thermal. 
Vibrational effects will be presented later, however this graph presents a good visual representation of the effects that a combination of perturbations has on the laser's output. When considering the jitter pertaining to engine operation, the variation of spark timing may change by as much as $\pm 2.9 \mathrm{CA}$ degrees.

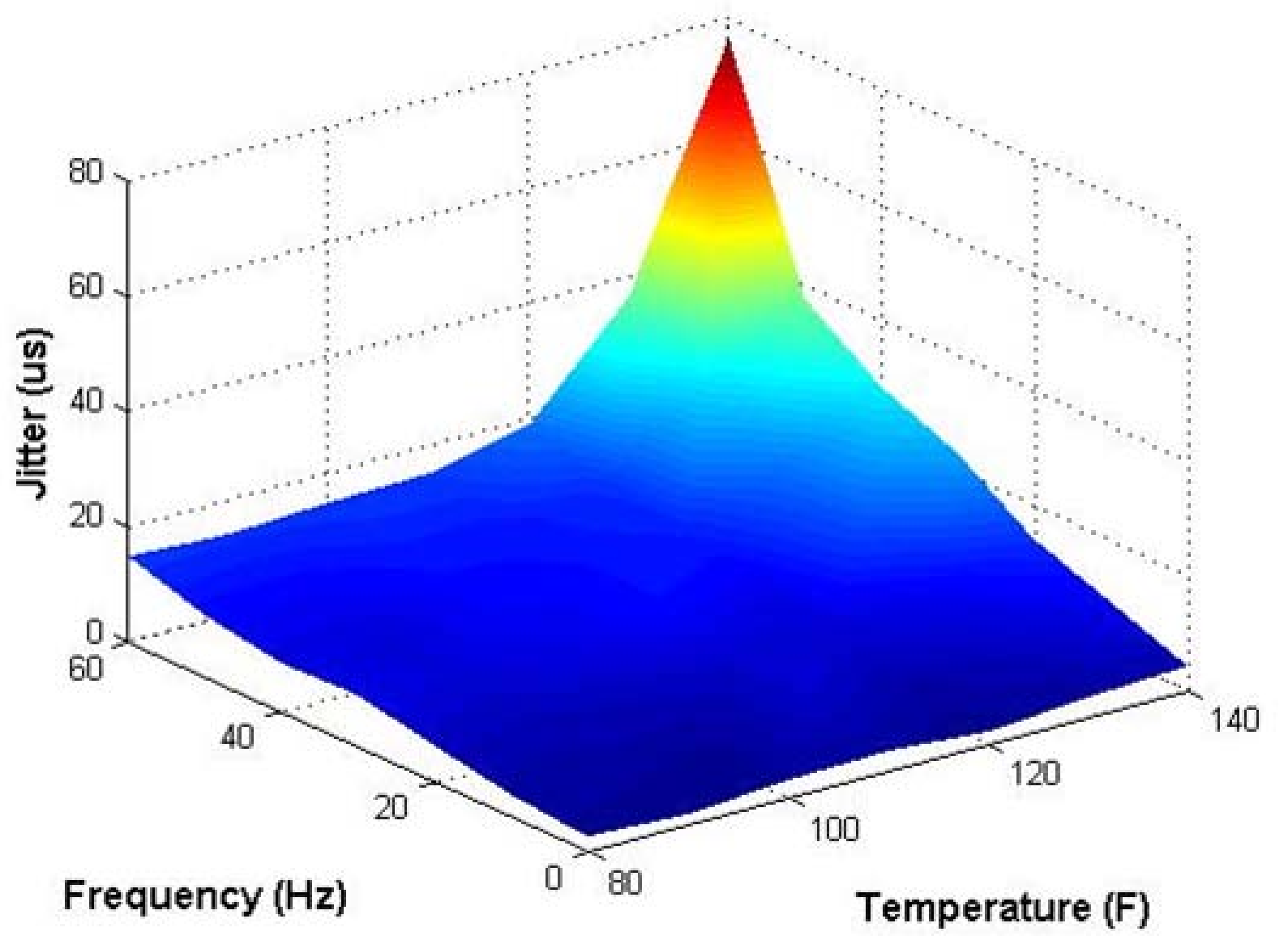

Figure 46: Average jitter as a function of frequency and temperature for the 90 degree mounting orientation with $100 \%$ acceleration

Again, the anticipated output parameter was not consistent for the 45 degree mounting orientation. As shown in Figure 47, the jitter was found to increase as a function of temperature for the 0 degree and 90 degree mounting orientations, while the jitter was found to remain relatively constant for the 45 degree mounting orientation. The same shot-to-shot deviation existed for this 45 degree mounting angle, however the magnitude at which it fluctuated was significantly less. Again, this is attributed to precision alignment. 


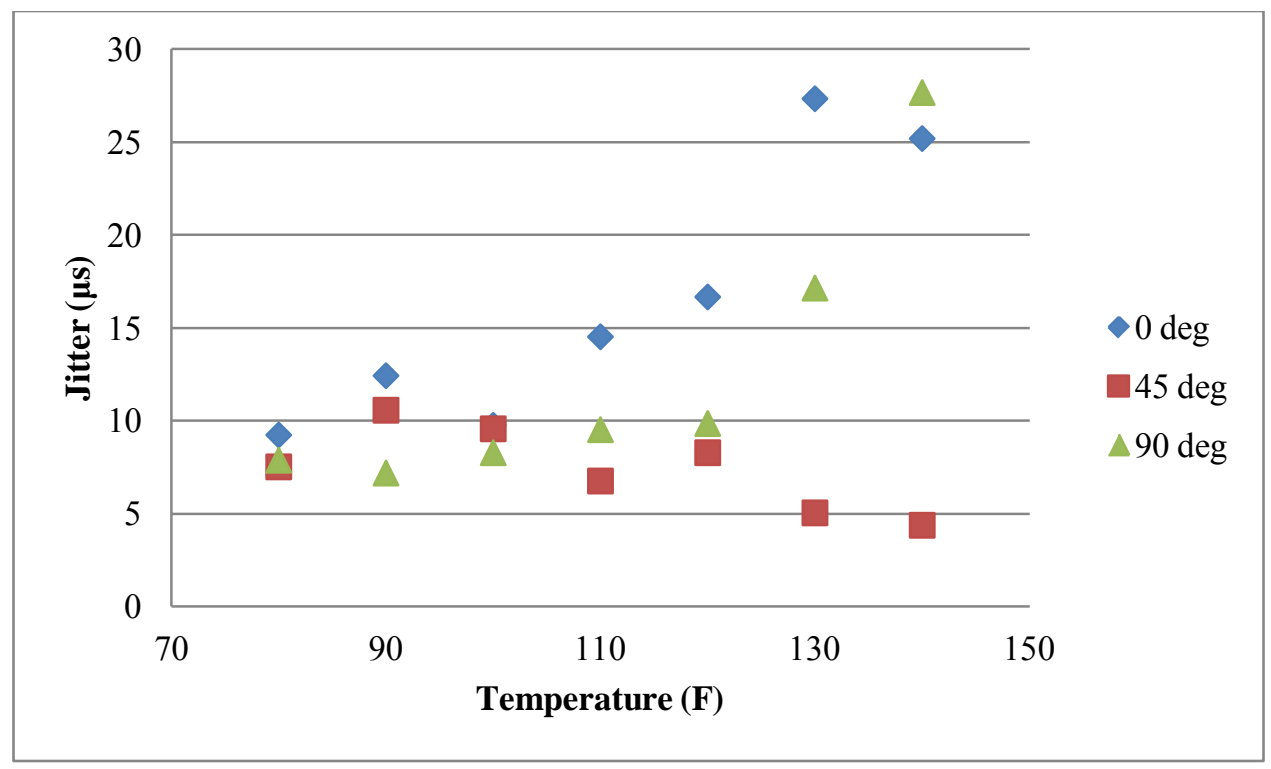

Figure 47: Average jitter as a function of temperature for the 0,45 and 90 degree mounting orientations with $100 \%$ acceleration

Jitter is a strong function of inherent heterogeneities within the gain medium and the saturable absorber, in addition to the coupling of random noise to the timing signal [59]. During each pulse there is a variation in the absorption rate for both the gain medium and the saturable absorber due to the molecular arrangement of the crystals. This variation exists not only due to the inhomogeneities in the physical structure of the crystals, but to heterogeneities and inconsistencies in the pumping light as well. For these reasons, there will always be an inherent amount of jitter present in a laser system. Certain laser arrangements produce less jitter than others under similar operating environments and in some cases, steps can be taken to minimize the jitter. To minimize jitter, a compact rigid design is necessary as to keep the optical path length as small as possible and to be as resistant as possible to thermal expansion.

As shown in Figure 48 where the jitter is analyzed for the 45 degree mounting orientation, vibrating at $60 \mathrm{~Hz}$ with $100 \%$ acceleration, a decrease in jitter as a function of temperature is observed. This result is not consistent with the other mounting orientations, nor is it consistent with the correction factor testing or the literature. 


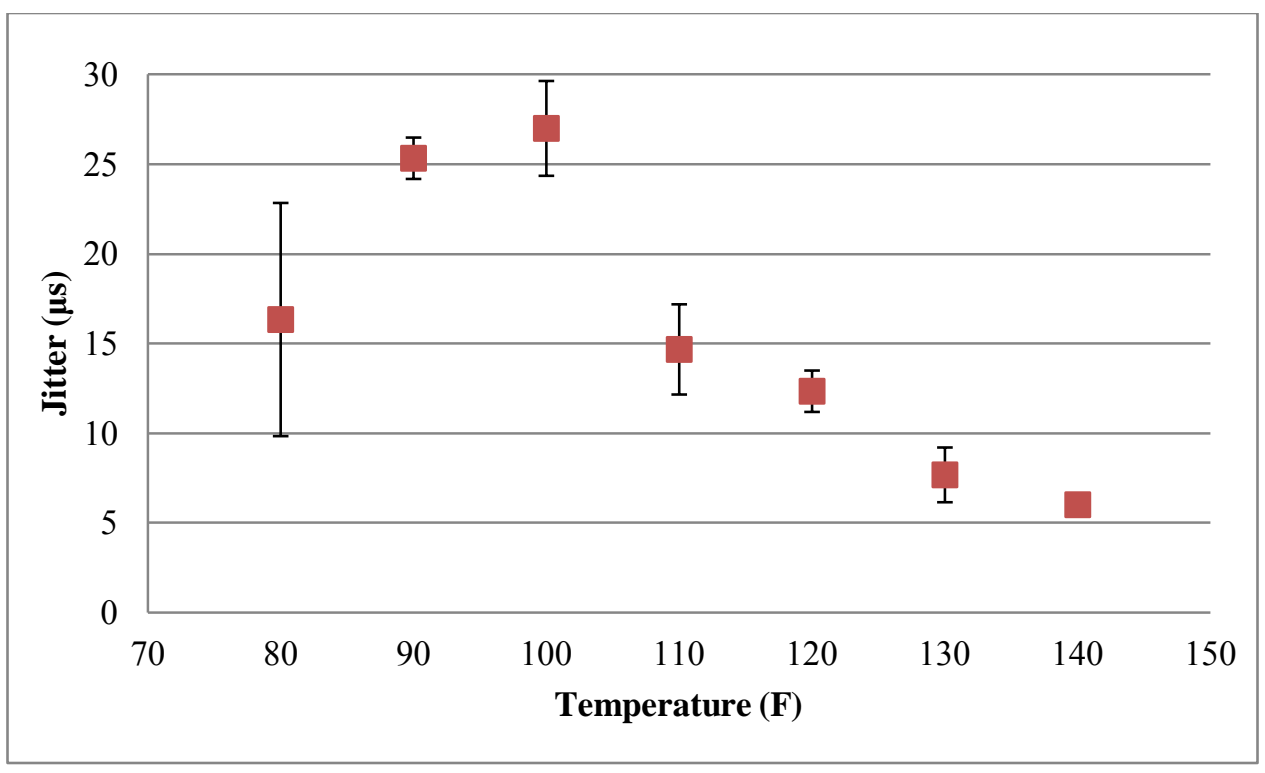

Figure 48: Jitter as a function of temperature in the 45 degree mounting orientation vibrating at $60 \mathrm{~Hz}$ with $100 \%$ acceleration

Not only is the magnitude of the jitter, shown in Figure 48, small compared to the other mounting orientations, but the bars representing one standard deviation are small as well. Oddly enough, the largest deviation in the jitter was found to occur at the lowest temperature setpoint. This is not consistent with the other mounting orientations or the correction factor investigation. Figure 49 show jitter as a function of temperature at the $60 \mathrm{~Hz}$ vibrational setpoint for each mounting orientation with $100 \%$ acceleration. Here it is shown that for the 0 degree and 90 degree mounting orientations, the jitter trends upward as temperature increases, however, as previously shown in Figure 48 and now in Figure 49 this upward trend does not occur in the 45 degree mounting orientation. Note the large scale increase in the jitter axis. For the $140{ }^{\circ} \mathrm{F}$ setpoint as shown in Figure 49, the magnitude of the measured jitter in the 0 degree mounting orientation was over 17 times larger than the magnitude of the jitter at the same thermal setpoint in the 45 degree mounting orientation. This may be because the 45 degree mounting orientation is the most complimentary mounting orientation in accommodating for thermal expansion thereby maintaining optical alignment. 


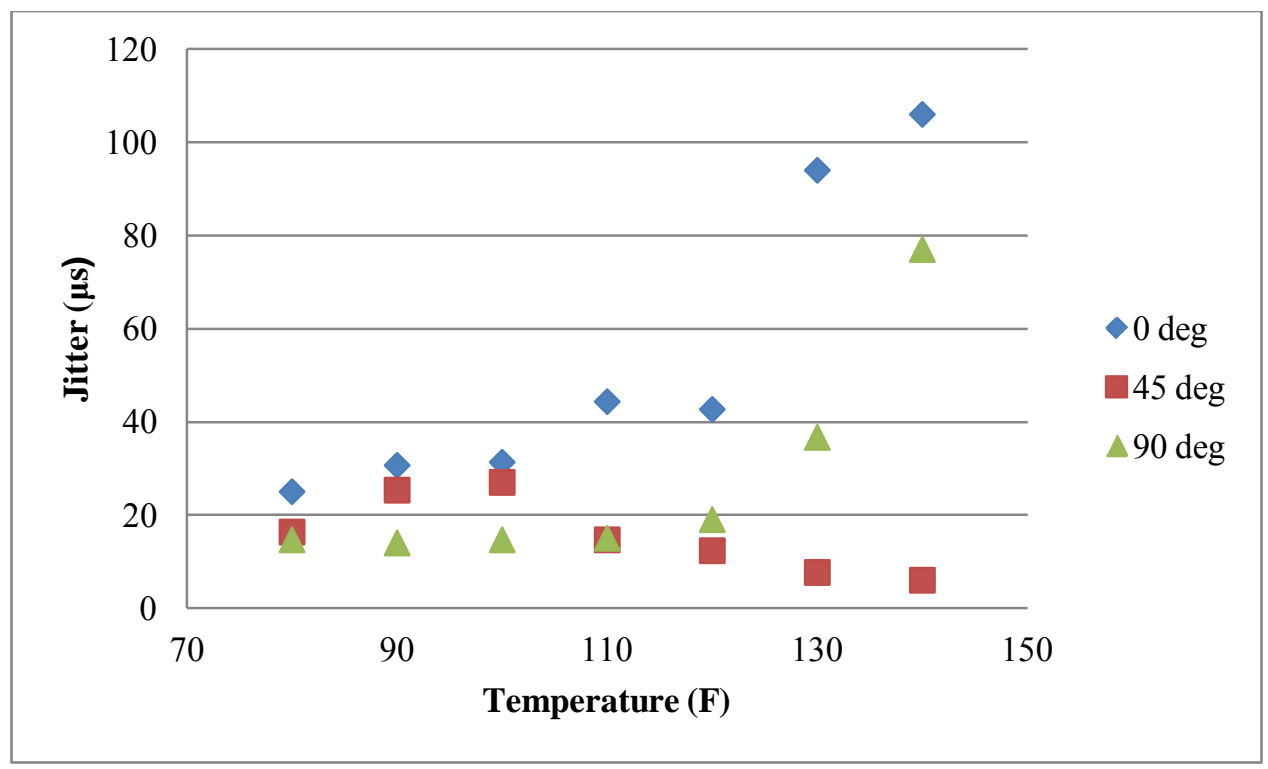

Figure 49: Jitter as a function of temperature at $60 \mathrm{~Hz}$ for the 0,45 and 90 degree mounting orientations with $100 \%$ acceleration

\subsubsection{Energy}

The energy that each laser pulse delivers into the combustion chamber has an impact on the ignition efficiency of the engine's power stroke. It has been shown in the literature that there exists a minimum amount of spark energy that is required to effectively ignite an air fuel mixture in the combustion chamber $[8,12,60,61]$. Alternatively there is an upper energy threshold that exists where if the ignition pulse energy exceeds this threshold the ignition efficiency does not change [61]. The point where the ignition energy reaches this upper energy threshold is considered to be the ideal ignition spark energy since this is the energy level that will yield efficient combustion while using the optimal amount of pulse energy. These upper and lower thresholds vary depending upon the properties of the fuel, and are described further in the literature $[60,61]$

In Figure 50, the average energy output as a function of temperature is displayed for each of the mounting orientations for the $50 \%$ acceleration setpoint. As shown, the mounting orientation with the highest variability in energy output is the 0 degree orientation. Relating to the correction factor data in Figure 30, it was shown that output energy increases as a function of increasing temperature. This still holds true for the data in Figure 50, however when averaged over the frequency band, the output energy does not appear to follow a particular trend for any of 
the thermal setpoints. The 0 degree mounting orientation is the most susceptible to vibrational influences since it is mounted as a cantilever. Thermal expansion of the lasers chassis causes the most deviation in this 0 degree mounting orientation primarily due to the force of gravity. For this orientation, several alignment procedures were conducted to compensate for this cantilever bending. However, when data was collected for each of these orientations, there was still a noticeable increase in output energy as a function of increasing temperature for each of the $0 \mathrm{~Hz}$ setpoints. This coincides with the fact that output energy increases as a function of temperature, and the deviation is a strong function of frequency, which will be discussed later in Section 5.3.5.

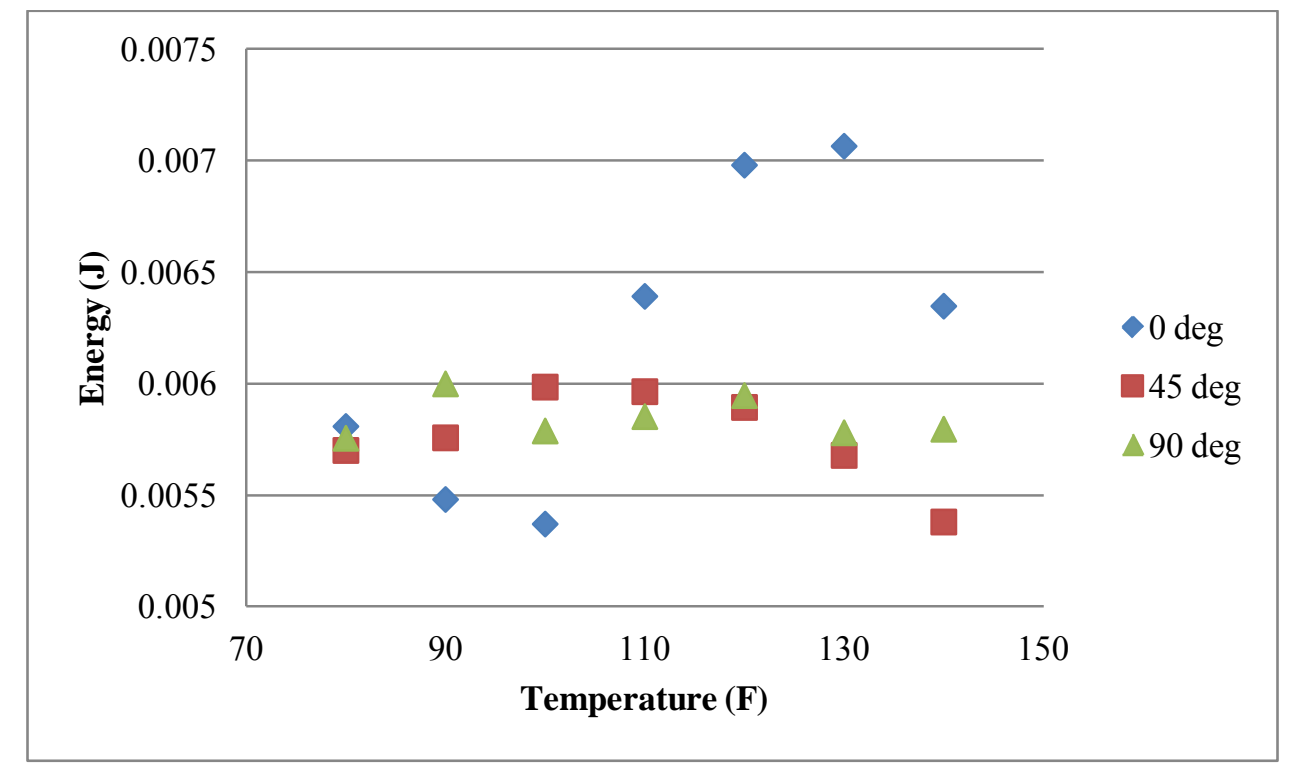

Figure 50: Average energy as a function of temperature for 0, 45 and 90 degree mounting orientations with $50 \%$ acceleration

For the 45 degree and the 90 degree mounting orientations as shown in Figure 50, the energy output remains relatively constant. In each of these cases, the output energy shows very little deviation. For example, Figure 51 shows the average output energy as a function of temperature in the 45 degree mounting orientation at $50 \%$ acceleration, it can be seen that the bars which represent one standard deviation from the mean are small. This is ideal when considering this optical setup for LI applications. The more steady and consistent the output energy remains as a function of increasing temperature the more consistent the focal intensities will be thus leading to more consistent combustion. 


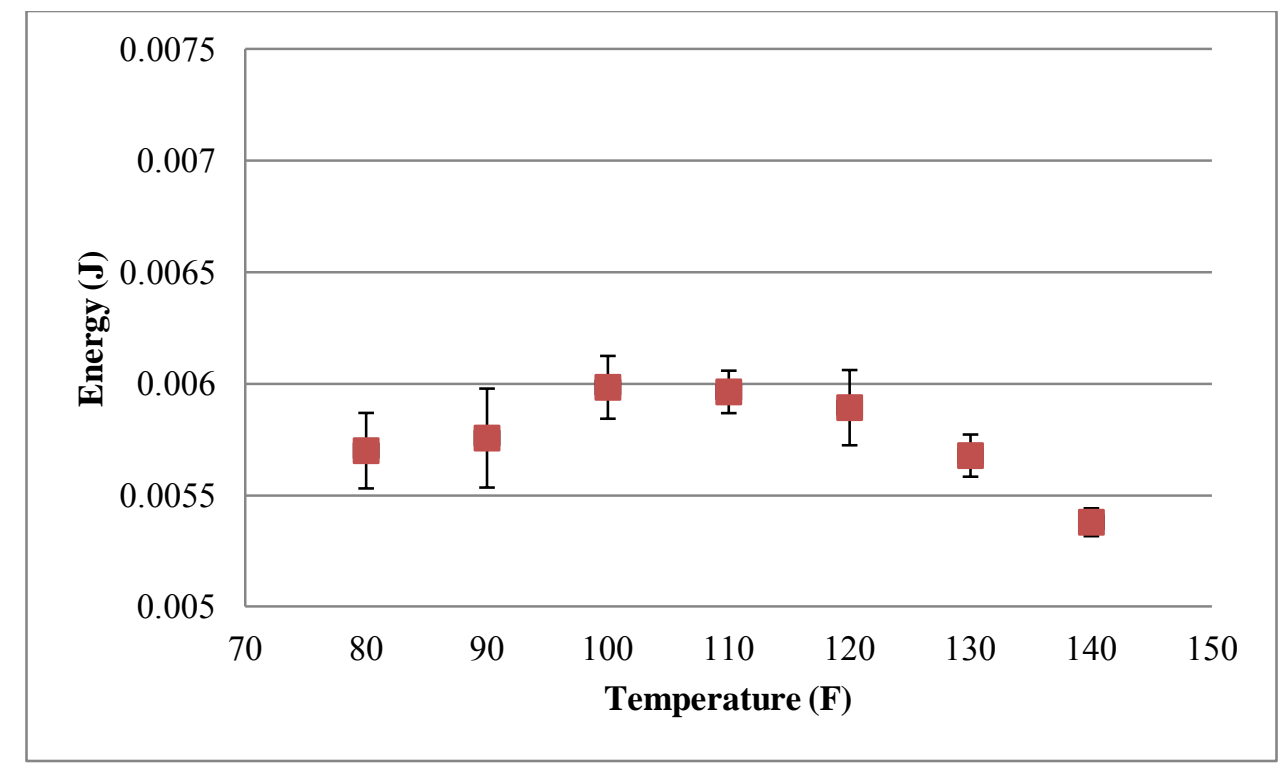

Figure 51: Average energy as a function of temperature for the 45 degree mounting orientation with $50 \%$ acceleration

As shown in Figure 52, the output energy as a function of temperature for the 90 degree mounting orientation show very little dependence upon acceleration. For each of the thermal data points collected, the $50 \%$ and $100 \%$ setpoints fall nearly on top of each other. The consistency between acceleration setpoints across the temperature range is consistent with expectations because the output energy is a function of temperature as shown in Equation 2-7 and not dependent upon the magnitude of vibrational frequency. Figure 52 shows the temperature dependence on the output energy, however the results presented here appear to be nearly constant across the thermal setpoints, which is not consistent with the literature. Figure 10 shows the output energy increasing with increasing temperature. An explanation for this is that the temperature range presented in Figure 10 is much larger than the temperature range presented here in Figure 52. The range of temperatures presented here may not be large enough to effectively show the energy dependence upon temperature 


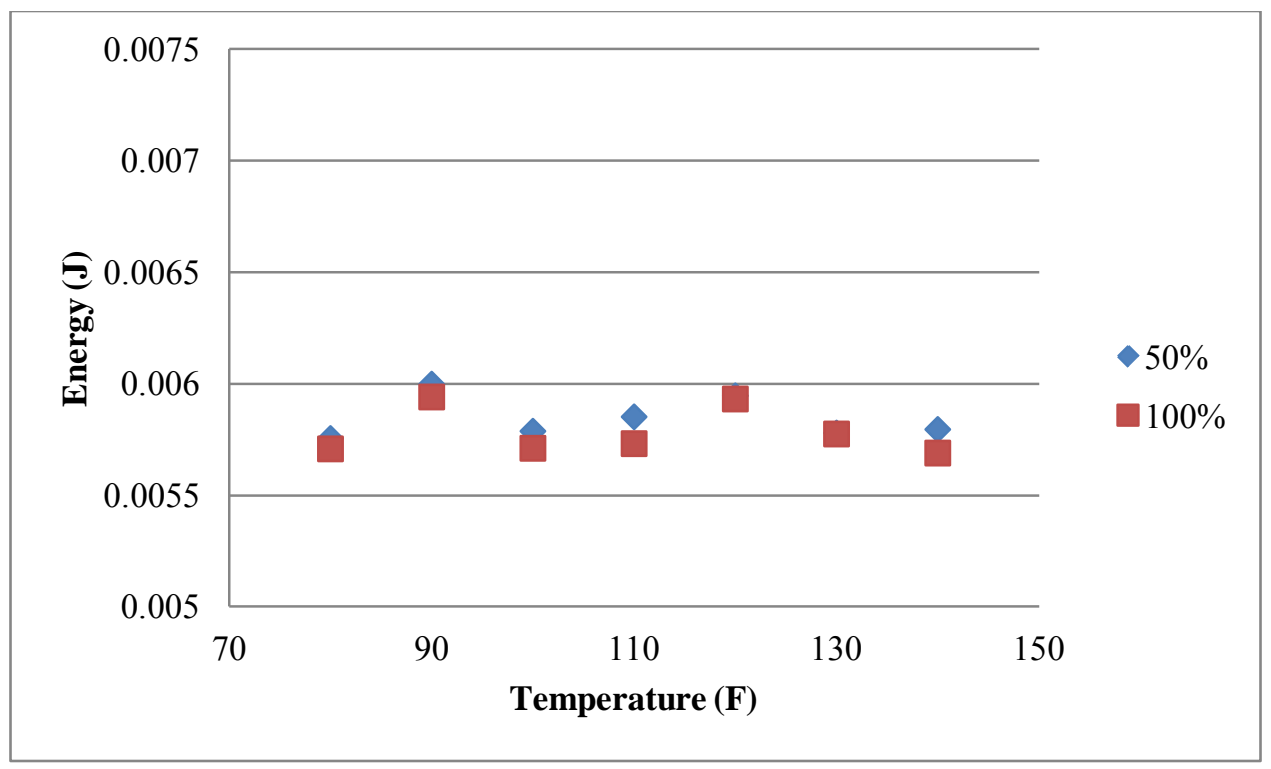

Figure 52: Average energy as a function of temperature for the 90 degree mounting orientation with $50 \%$ and $100 \%$ acceleration

A good result is the measured output energy presented here when compared to the output energy measured in previous experimentation by McIntyre et al. In Figure 53 where the output energy is shown as a function q-switch initial transmission percentage, it can be seen that the resulting output energy at $10 \%$ initial transmission is roughly equivalent the measured energy presented here. This result was as to be expected. Although a direct comparison of numerical findings may not be reasonable since a $20 \%$ OC was used in this investigation, the results from McIntyre et al. appear to be consistent with the results presented here. 


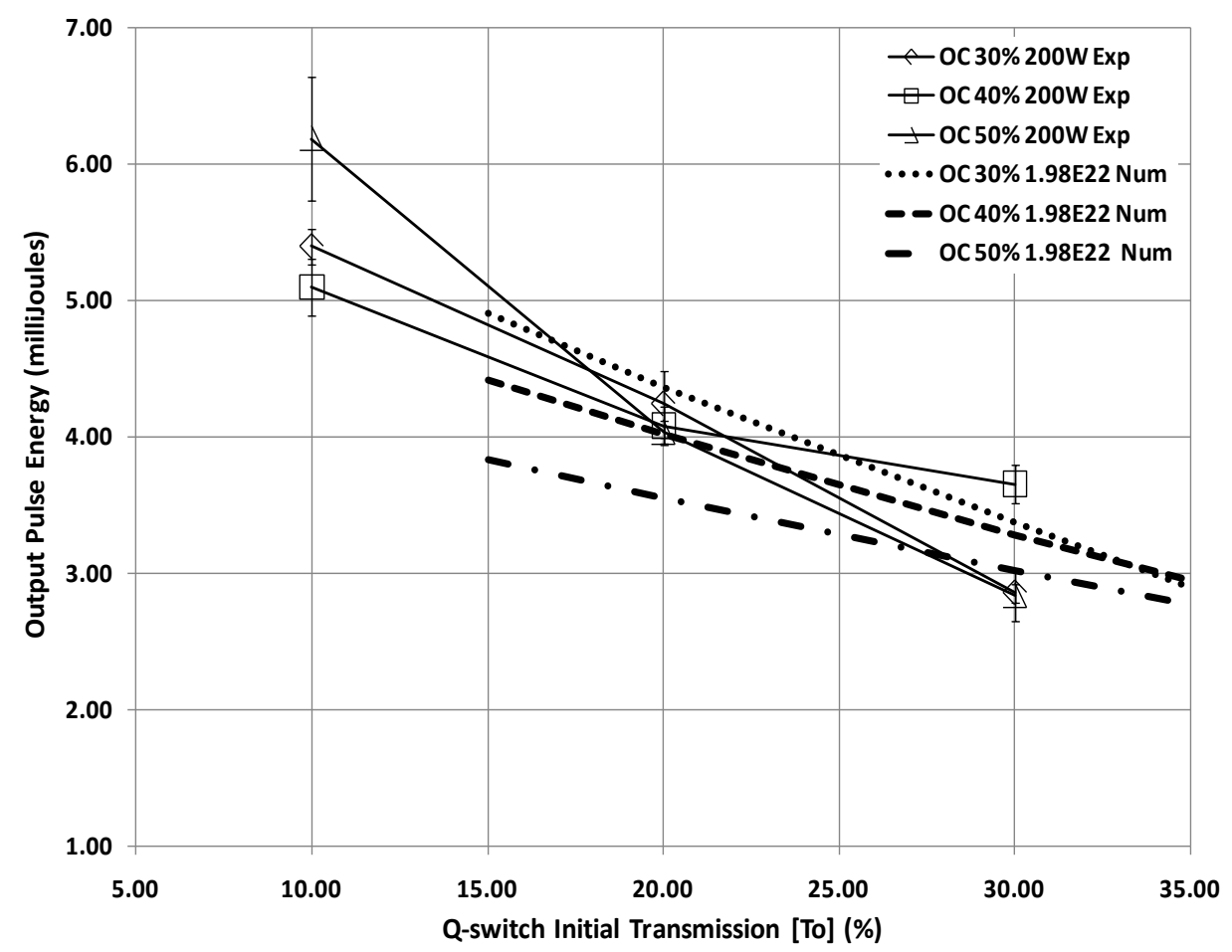

Figure 53: Output energy as a function of q-switch initial transmission percentage [29]

\subsubsection{Focal intensity}

As previously discussed, the focal intensity is a function of output energy, pulse width and focal area as defined in Equation 5-4. The trend of Figure 54 is similar to the trend in Figure 50 meaning that the output energy is the primary factor in determining focal intensity. This is primarily due to the small magnitudes of changing pulse width as seen in Figure 36 and that the focal area was assumed constant across the array of test matrices. As shown Figure 54, each of the focal intensity data points when averaged over the vibrational frequency band is above the breakdown threshold of $100 \mathrm{GW} / \mathrm{cm}^{2}$ making every data point conducted in this testing capable of generating a plasma spark. Recall from Section 2.2 that the breakdown threshold for gas decreases as a function of increasing pressure as well. This indicates that as LSP is currently capable of achieving ignition inside an engine's combustion chamber at elevated pressures. 


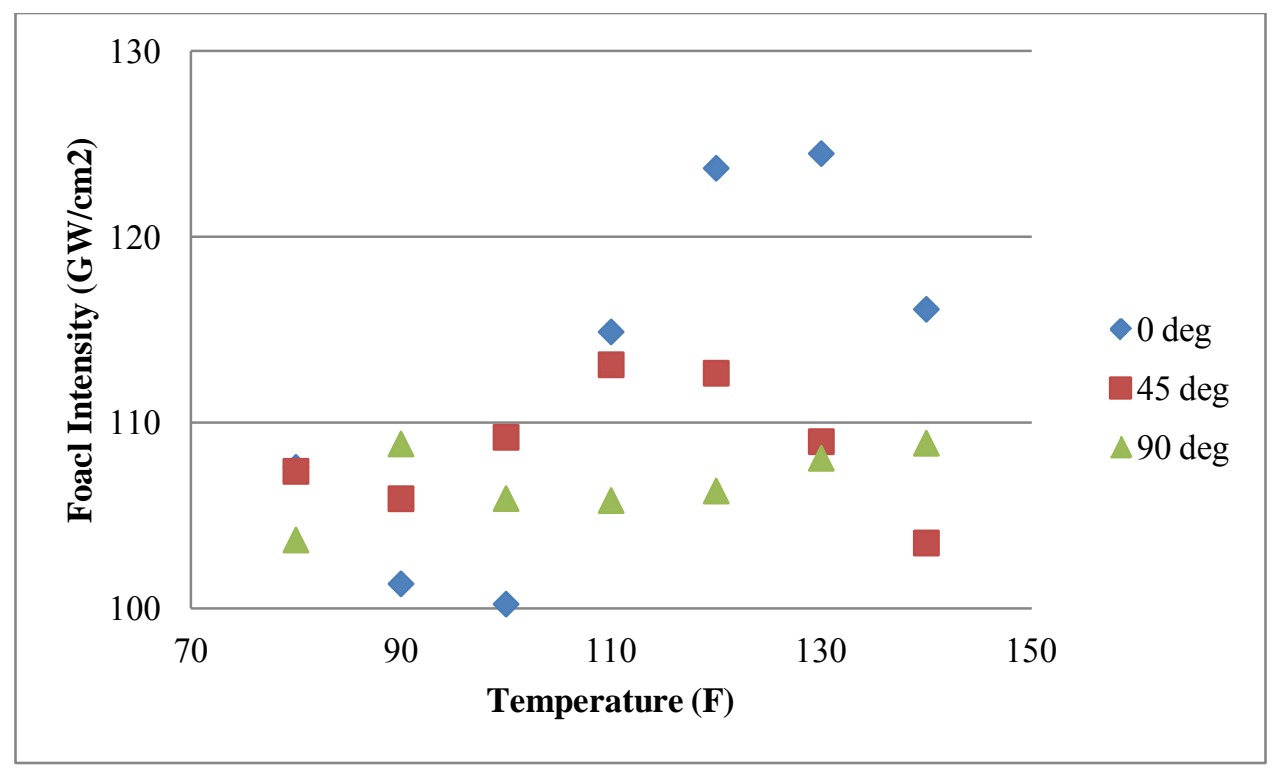

Figure 54: Focal intensity as a function of temperature for the 0,45 and 90 degree mounting orientations with $50 \%$ acceleration

Although every focal intensity data point that was calculated was intense enough to generate a plasma spark in air, the variation measured in the focal intensity when averaged over the frequency band is significant. The focal intensity was found to vary by as much as 25 $\mathrm{GW} / \mathrm{cm}^{2}$ over a range of temperatures, which may result in a variation of ignition and combustion efficiency. In an ideal case, the focal intensity would remain near constant as to ensure consistent ignition events.

\subsection{Vibration}

The influence of vibration was found to have a profound impact on the output pulse produced by the LSP. Pulse width, pulse width variation, Q-switch delay, jitter and energy output were all found to vary with respect to oscillatory perturbations. The variation of these parameters will lead to cycle-to-cycle variation within the combustion chamber through mechanisms such as ignition delay, ignition timing variation and ignition efficiency. The following describes the vibrational dependence of the LSP on the independent variables collected from the oscillatory and thermal testing. 


\subsubsection{Pulse Width}

The output pulse width of the ignition laser has a significant impact on the on the ability to generate a plasma spark and its ability to initiate ignition. When increasing frequency, the mean pulse width was found to remain relatively constant as shown in Figure 55 for each mounting angle configuration for the $100 \%$ acceleration test points. Similar trends are found for the $50 \%$ acceleration setpoints (Appendix-E) indicating that not only is the pulse width of the laser output virtually independent of acceleration and amplitude, but it is widely independent of vibrational frequency as well. In Figure 55, it can also be seen that there is approximately a 0.1 ns time reduction in the output pulse width for the 45 degree mounting orientation. A reduction in the pulse width will yield a higher focal intensity as previously defined in Equation 5-4. This reduction may be due to achieving a slightly better alignment for the 45 degree mounting orientation or due to rotation of the intracavity components upon installation after they were removed for periodic cleaning.

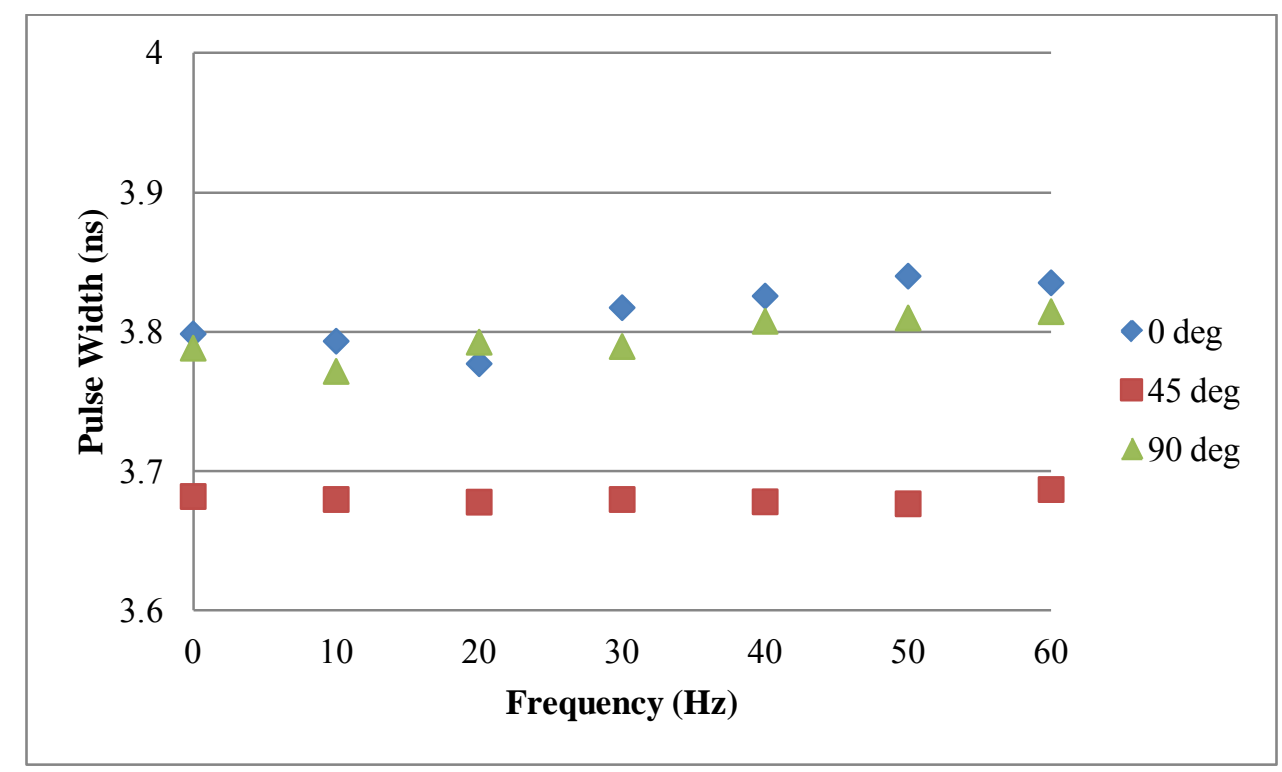

Figure 55: Average pulse width as a function of frequency for the 0, 45 and 90 degree mounting orientations with $100 \%$ acceleration

When analyzing a single mounting angle with bars representing one standard deviation in addition to the pulse width variation in Figure 56, it is shown that the average pulse width for 
each frequency setpoint falls within the margin of variation represented here. Given the minute scale of variation at each frequency setpoints, it can be stated that the pulse width stays relatively constant with respect to frequency. Using this assumption, the focal intensity for each mounting angle becomes strictly a function of output energy. The values for focal intensity presented later in Section 5.3.6 still utilize the averaging method for the values presented here in Figure 55, however since these values are averaged over the temperature band and there is little change in the pulse width, it can be said that pulse width is independent of vibrational frequency and pump pulse frequency.

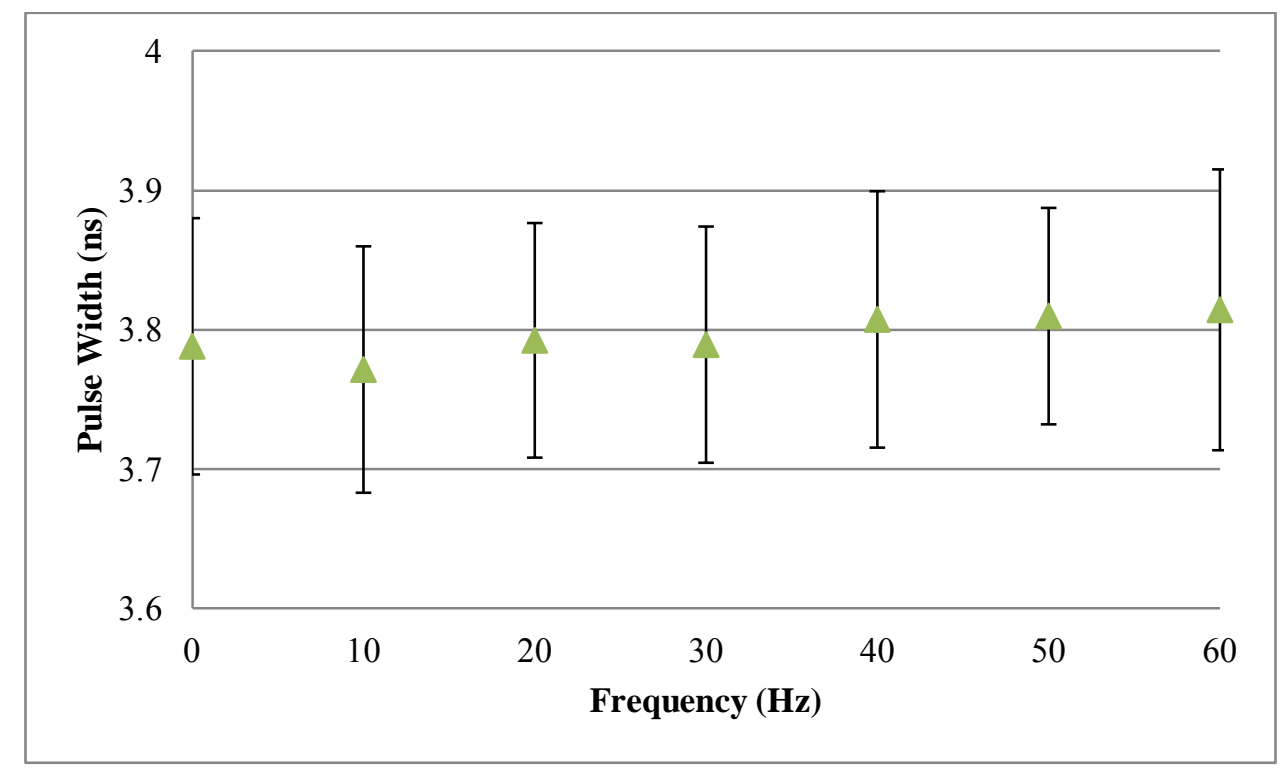

Figure 56: Average pulse width as a function of frequency for the 90 degree mounting angle with $100 \%$ acceleration

Significant cycle-to-cycle variations in the pulse width exist as a function of vibrational frequency as shown by the standard deviation bars in Figure 56. A significant difference in pulse width can mean the difference between generating a plasma spark or not. A means of minimizing this variation in the pulse width is necessary to ensure consistent ignition parameters. Fueling and ignition strategy must be controlled based operating pulse width of the LSP. To ensure consistent ignition results, fixing the pulse width and minimizing its variation is necessary. As discussed next in Section 5.3.2, the pulse width variation with respect to vibrational frequency has the potential to be reduced with an alternative configuration of pump steering optics and intracavity components. 
An interesting result that should be mentioned is the measured output pulse width presented here when compared to the output pulse width measured in previous experimentation by McIntyre et al. In Figure 57 where the output pulse width is shown as a function q-switch initial transmission percentage, it can be seen that the resulting pulse width at $10 \%$ initial transmission is roughly half the measured pulse width presented here. This may be due to the lower transmission OC (20\%) used in this investigation, or a better alignment being obtained leading to a higher coupling efficiency.

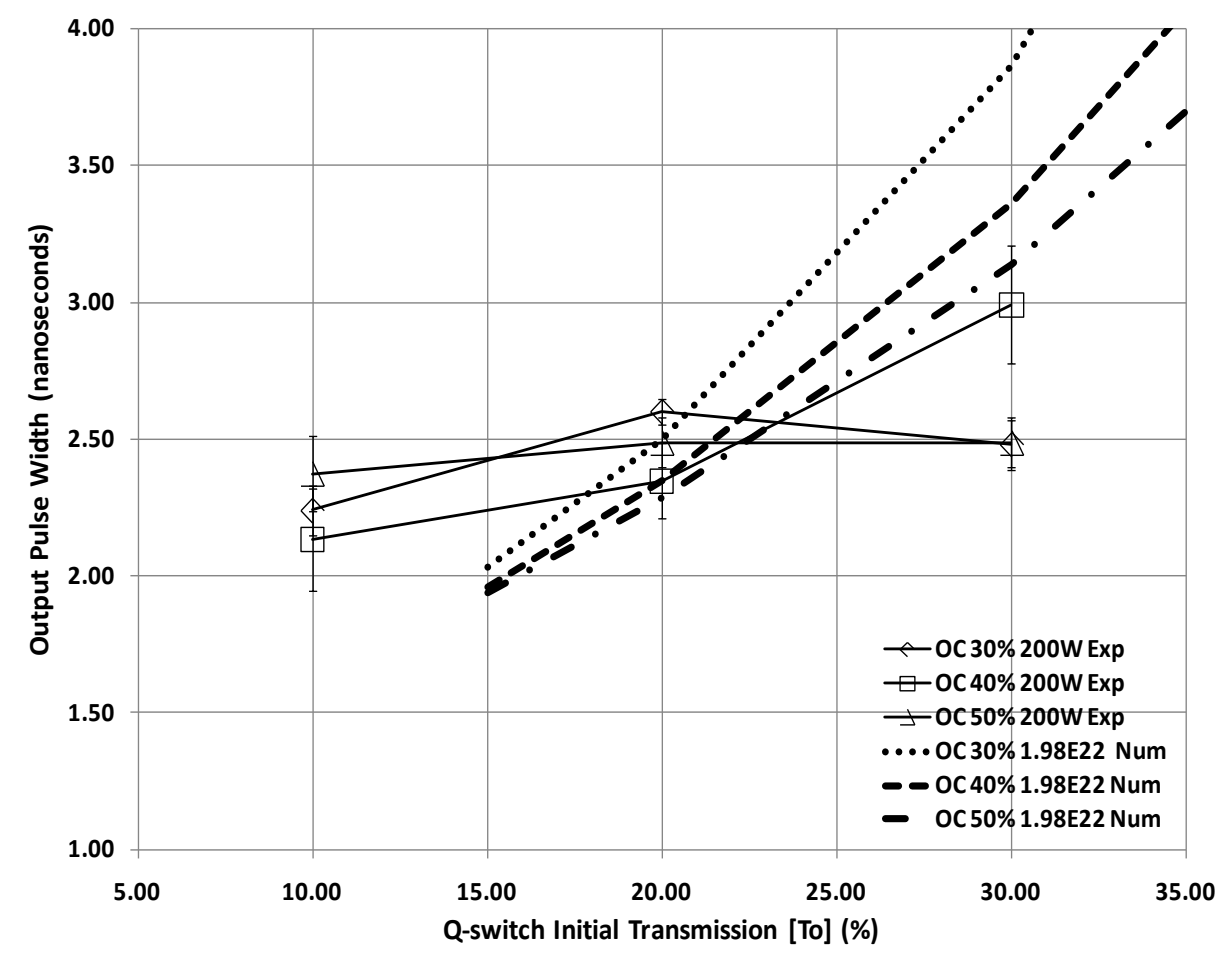

Figure 57: Output pulse width as a function of q-switch initial transmission [29]

\subsubsection{Pulse Width Variation}

Pulse width variation has the potential to cause cycle-to-cycle variation within the system. With increasing vibrational frequency, the variation in the pulse width has been found to vary for each mounting angle. Although the results show variation on the tens of picoseconds scale, there exists room for improvement. Figure 58 shows the average pulse width variation as a function of vibrational frequency for each mounting orientation in the $50 \%$ acceleration setpoints. 


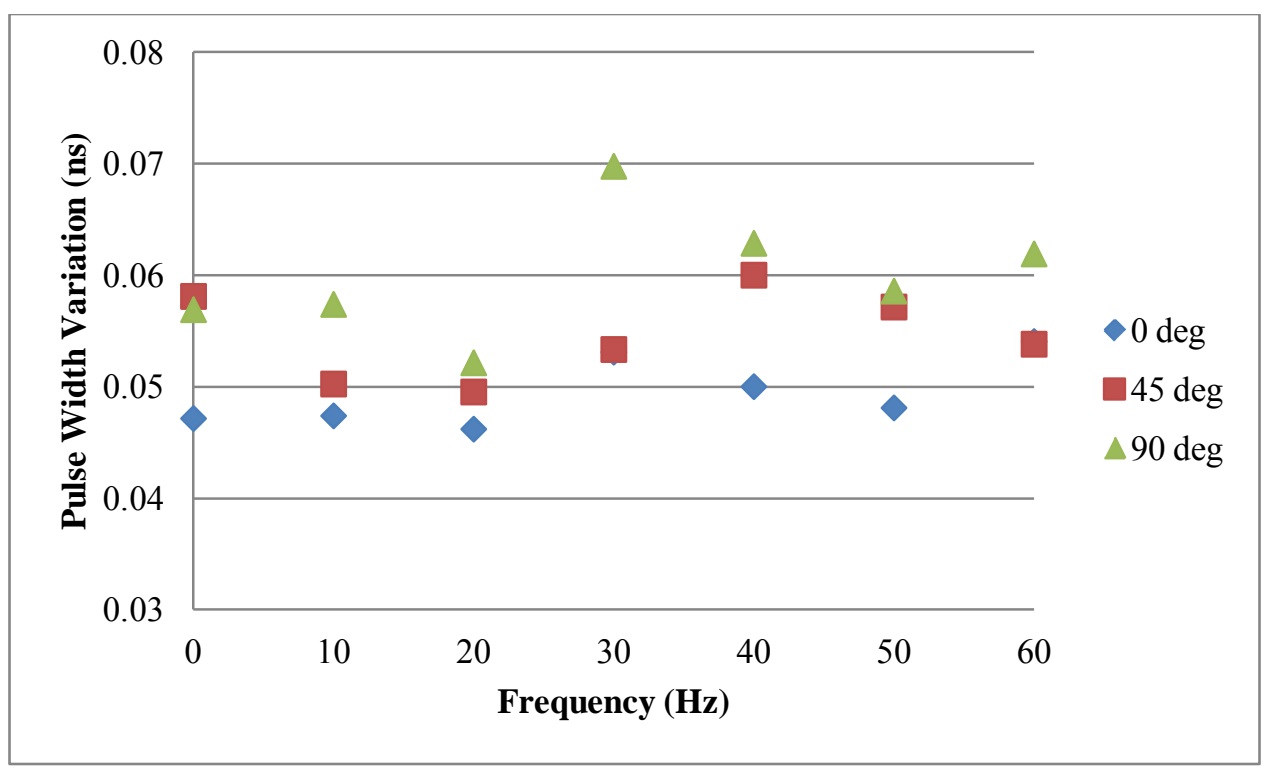

Figure 58: Average pulse width variation as a function of frequency for the 0,45 and 90 degree mounting angles with $50 \%$ acceleration

As shown below in Figure 59 where the average pulse width variation is represented with bars as a function of vibrational frequency for the 45 degree mounting orientation at the $50 \%$ acceleration setpoints, the magnitudes of the bars are of interest. If the standard deviation error bars on Figure 58 were to be added, the range of variation of the pulse width would encompass nearly every calculated average. Given the overlapping of measured variation, it may be assumed that the pulse width variation presented here is not a function of mounting angle and vibrational frequency. The variation in the trends is attributed to different vibrational harmonics of the LSP for a given mounting condition. 


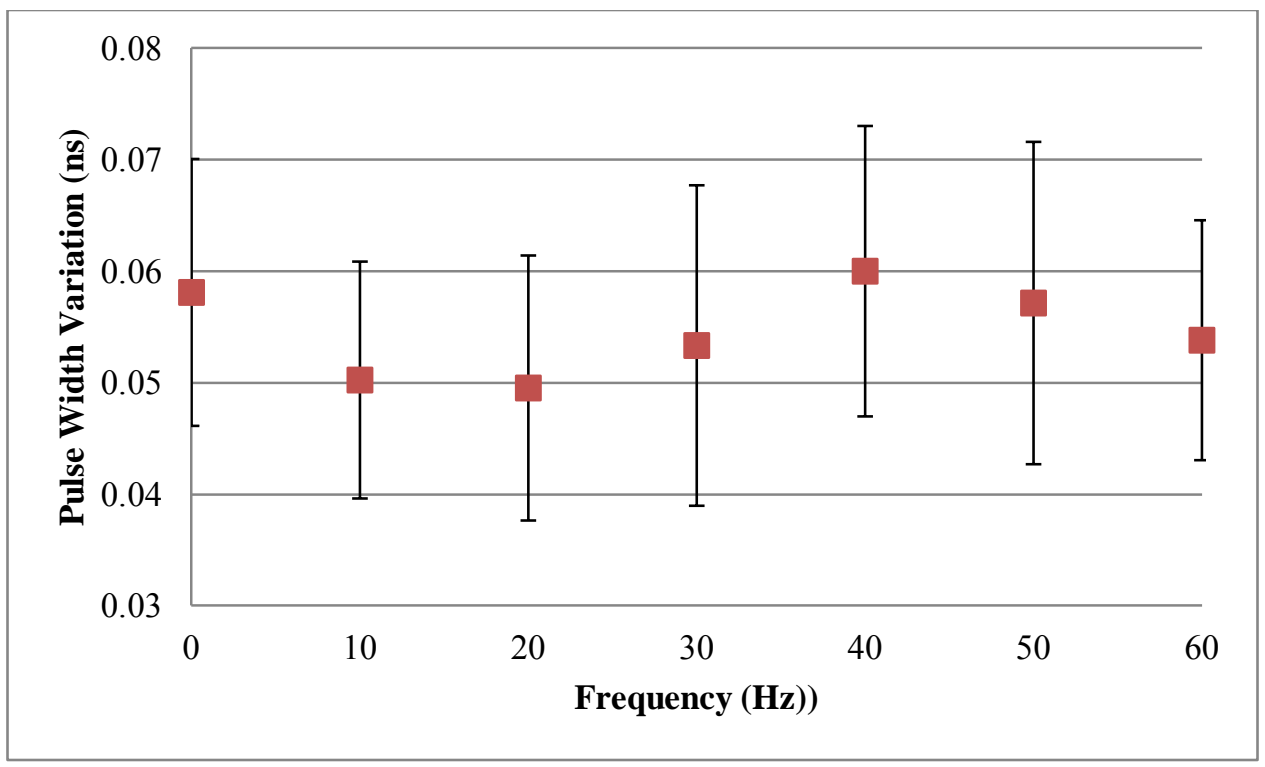

Figure 59: Average pulse width variation as a function of frequency for the 45 degree mounting orientation with $50 \%$ acceleration

Although not presented here, the vibrational harmonics of the physical structure of the payload were very apparent during testing, especially for the 0 and 45 degree mounting orientation. The harmonics of the LSP in the 90 degree orientation were not as apparent since the LSP was oriented coaxially with the oscillator. For the 0 and 45 degree mounting orientations, considerable shaking occurred for certain vibrational frequencies. At certain frequencies, the resulting induced vibration came close to the natural harmonic frequencies of the laser chassis. This was visually apparent during testing because at certain frequencies, nodes in the structure were noticed such that high rates of gyration would exist with very little linear acceleration. As distance from the node along the axis of the LSP increased, the local acceleration increased and the rate of gyration decreased. Although the accelerometer used here in this experimentation was mounted on the oscillator to LSP adapter, Figure 22 shows how the previously discussed induced acceleration varies as a function of vibrational frequency.

\subsubsection{Q-switch Delay}

The q-switch delay was averaged over the thermal setpoints and presented as a function of vibrational frequency in Figure 60 for the 0, 45, and 90 degree mounting orientations for the $100 \%$ acceleration setpoints. Well defined trends in q-switch delay for each of the mounting 
orientations can be seen. As expected from the correction factor investigation where q-switch delay was found to increase as a function of pump pulse frequency (shown in Figure 33), the qswitch delay for the 0 degree and 90 degree mounting orientations were found to increase as a function of frequency as well. This does not mean that q-switch delay increases as a function of vibrational frequency, but rather that the q-switch delay increases as a function of pump pulse frequency. A higher average heat deposition occurs for higher pumping frequencies. Recall that the pump pulse frequency increases at a rate of exactly half of the vibrational frequency setpoint.

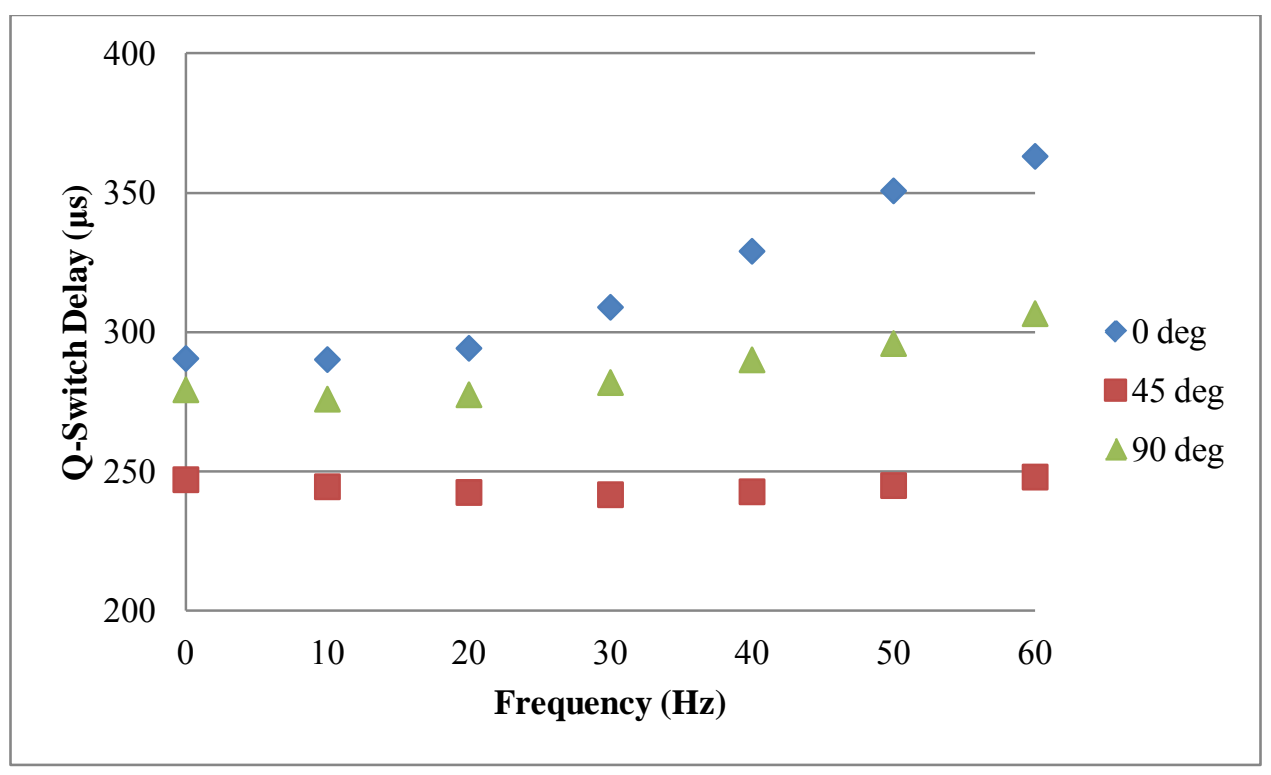

Figure 60: Average q-switch delay as a function of frequency for the 0,45 and 90 degree mounting orientations with $100 \%$ acceleration

Similar to the trend in q-switch delay as a function of temperature data for the 45 degree mounting angle presented in Figure 40, the q-switch delay presented in Figure 60 shows the 45 degree mounting orientation to remain nearly constant throughout the vibrational frequency range. This result contradicted the preliminary data found in the correction factor investigation. An explanation for this would be that in this mounting orientation, the physical structure of the LSP was able to handle the induced vibration in such a manner that it did not transfer the vibrational energy to susceptible components.

Figure 61 shows the q-switch delay as a function of vibrational frequency for the 90 degree mounting angle for the $100 \%$ acceleration setpoints with bars that represent one standard deviation from the mean in addition to the jitter. This graph was chosen because not only does it 
show the increase in q-switch delay, but it also shows how the variation in the q-switch delay increases as a function of vibrational frequency. For this mounting angle, the margin of variation is similar to that found with the q-switch delay as a function of temperature in Figure 41 . The qswitch delay increases as a function of pump pulse frequency which coincides with the literature and the correction factor investigation. The margins of variation of the delay increase as a function of vibrational frequency as well, which is as to be expected since as vibrational frequency increases, the alignment of the payload becomes more variable.

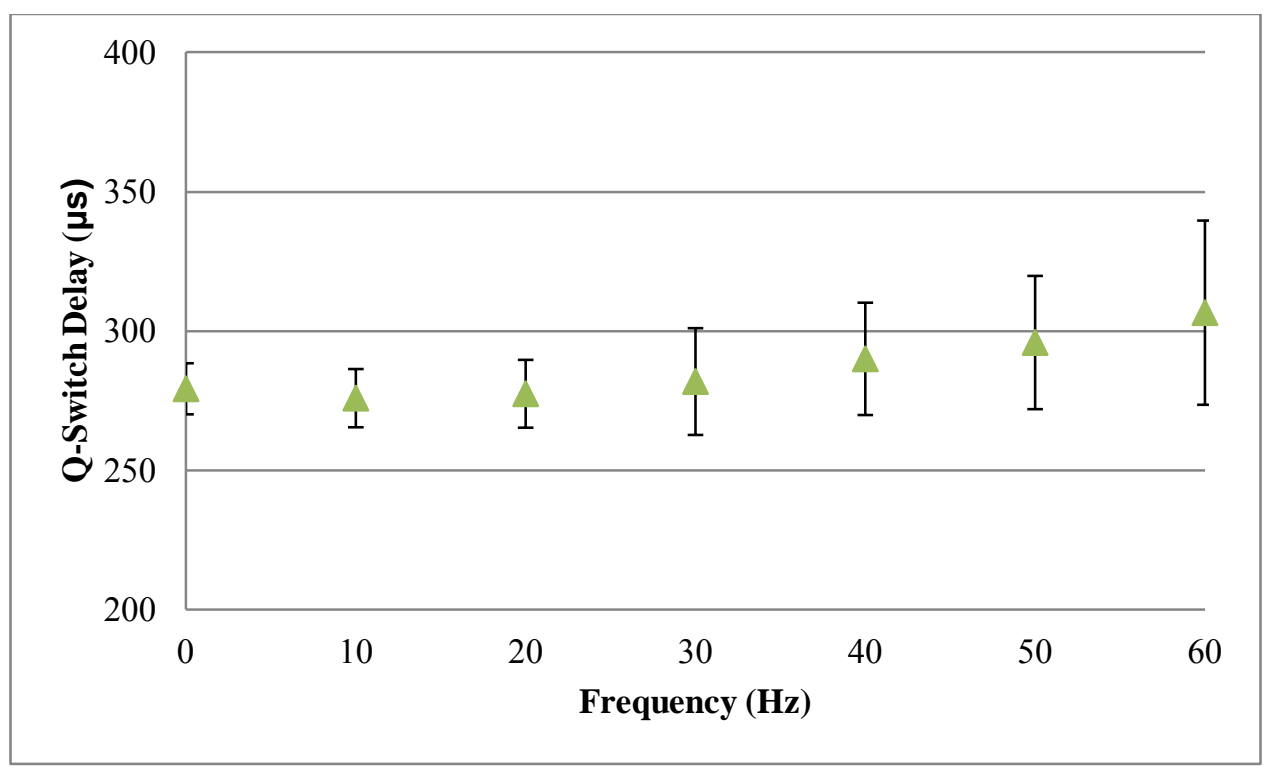

Figure 61: Average q-switch delay as a function of frequency for the 90 degree mounting orientation with $100 \%$ acceleration

Figure 62 shows the q-switch delay as a function of vibrational frequency for the 45 degree mounting orientation at the $100 \%$ acceleration setpoints. Although the trend of the average q-switch delay remains fairly constant, which is not consistent with the other mounting orientations, the margins of variation for the 45 degree mounting orientation was still found to increase as a function of vibrational frequency. The increasing margins of error as a function of increasing frequency are further discussed and explained in Section 5.3.4. 


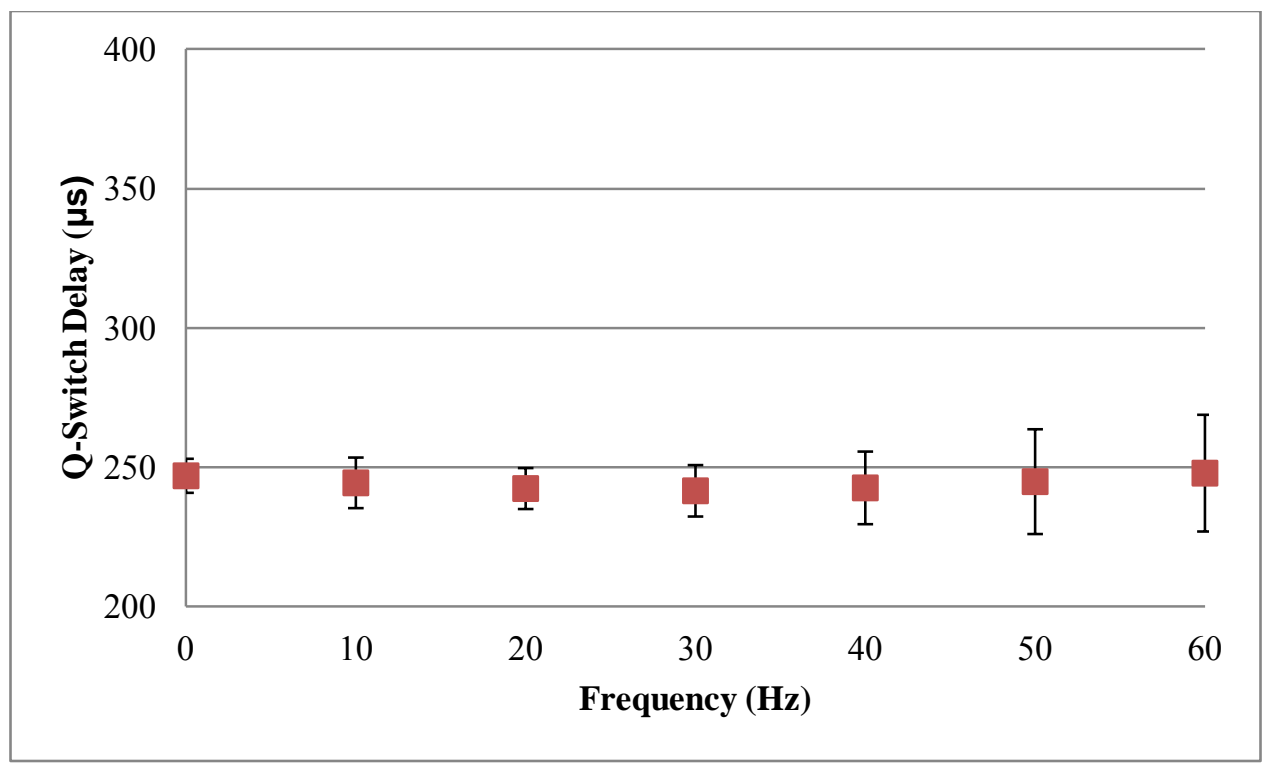

Figure 62: Average q-switch delay as a function of frequency for the 45 degree mounting orientation with $100 \%$ acceleration

For the purposes of this discussion, the q-switch delay is directly proportional to the ignition delay. Figure 63 shows how the ignition delay increases with respect to both temperature and vibrational frequency. This is a good graphical representation of how temperature and vibration both affect the delay of ignition. As shown, the point where the longest q-switch delay occurs is in the most extreme vibrational and thermal setpoint. The rate of ignition delay time increases gets becomes larger as the thermal setpoint is increased. This indicates that temperature and vibrational frequency both have a significant impact on the qswitch delay. Given this plot, an ignition control strategy may be realized to accurately control the ignition timing as a function of both of these independent variables. An ignition control strategy is needed because upon analysis of these results, it was determined that the spark timing may change by as much as $\pm 4.6 \mathrm{CA}$ degrees from the least extreme setpoint to the most extreme. 


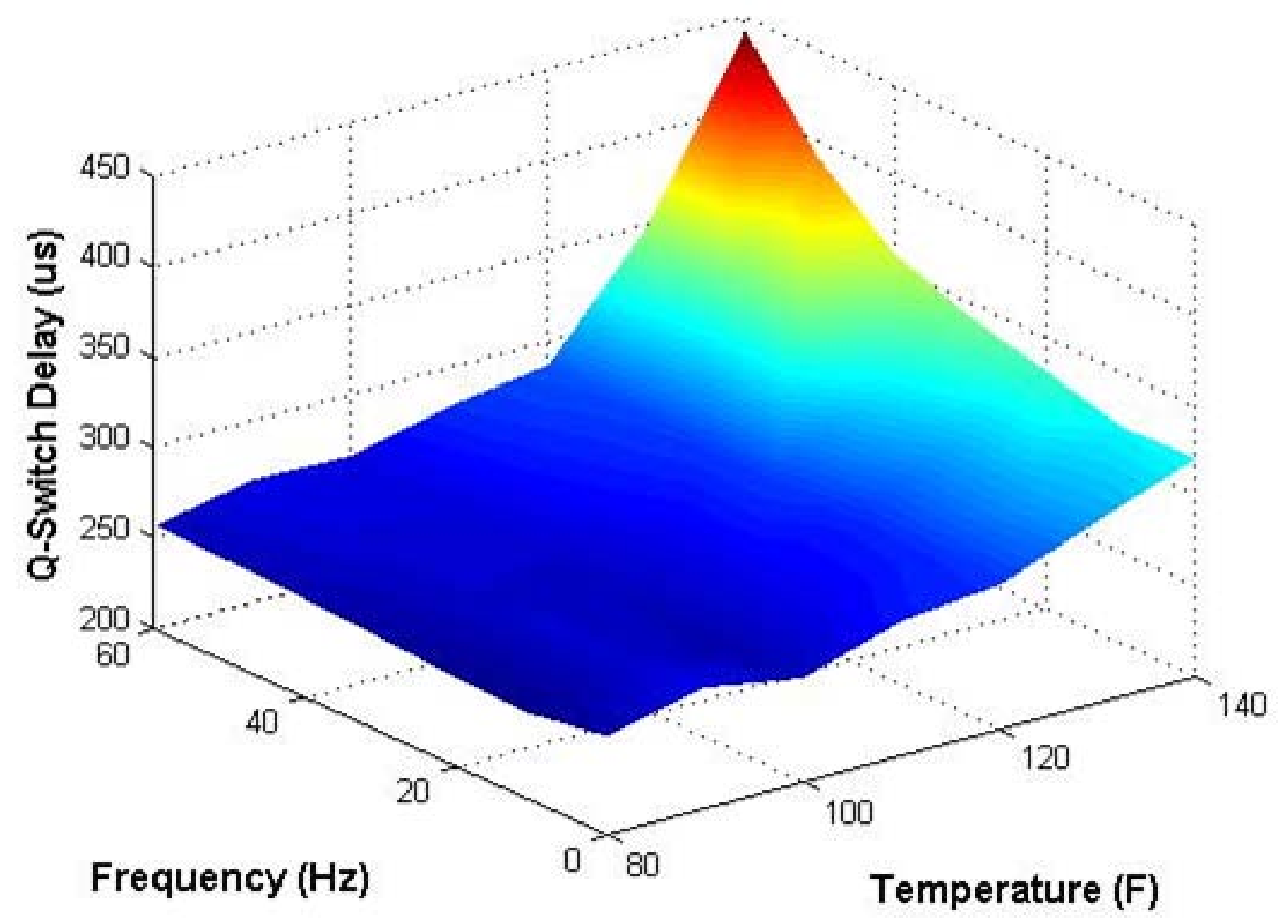

Figure 63: Average q-switch delay as a function of frequency and temperature for the 90 degree setpoint with $100 \%$ acceleration

\subsubsection{Jitter}

The jitter of the laser output is an important operating parameter of a LI system which affects the ignition delay as well as the variation in ignition timing. The more jitter that is present in an optical system the more diverse the cycle-to-cycle variation will be. Jitter may lead to combustion instabilities, as well as a decrease in the time averaged ignition efficiency. Jitter is an unattractive quality for this application, however a small amount of jitter is acceptable. Relating to IC engines, the jitter is best described in terms of change in CA on a cycle-to-cycle basis. This conversion is shown below in Equation 5-5 where $f$ is the applied vibrational frequency $(\mathrm{Hz}), 2 \delta$ is twice the measured jitter (s), $\omega$ is the corresponding rotational velocity of the engine (RPM) and a 360 constant conversion factor (deg/rev). 
Figure 64 shows the average jitter as a function of vibrational frequency for the 90 degree mounting orientation for the $50 \%$ and $100 \%$ acceleration setpoints. The jitter was found to increase as a function of vibrational frequency regardless of the imposed acceleration. The faster the laser is shaken, the higher the probability for variation of the coupling efficiency. This result is consistent with the literature and is one of fundamental issues regarding mounting a laser on an engine.

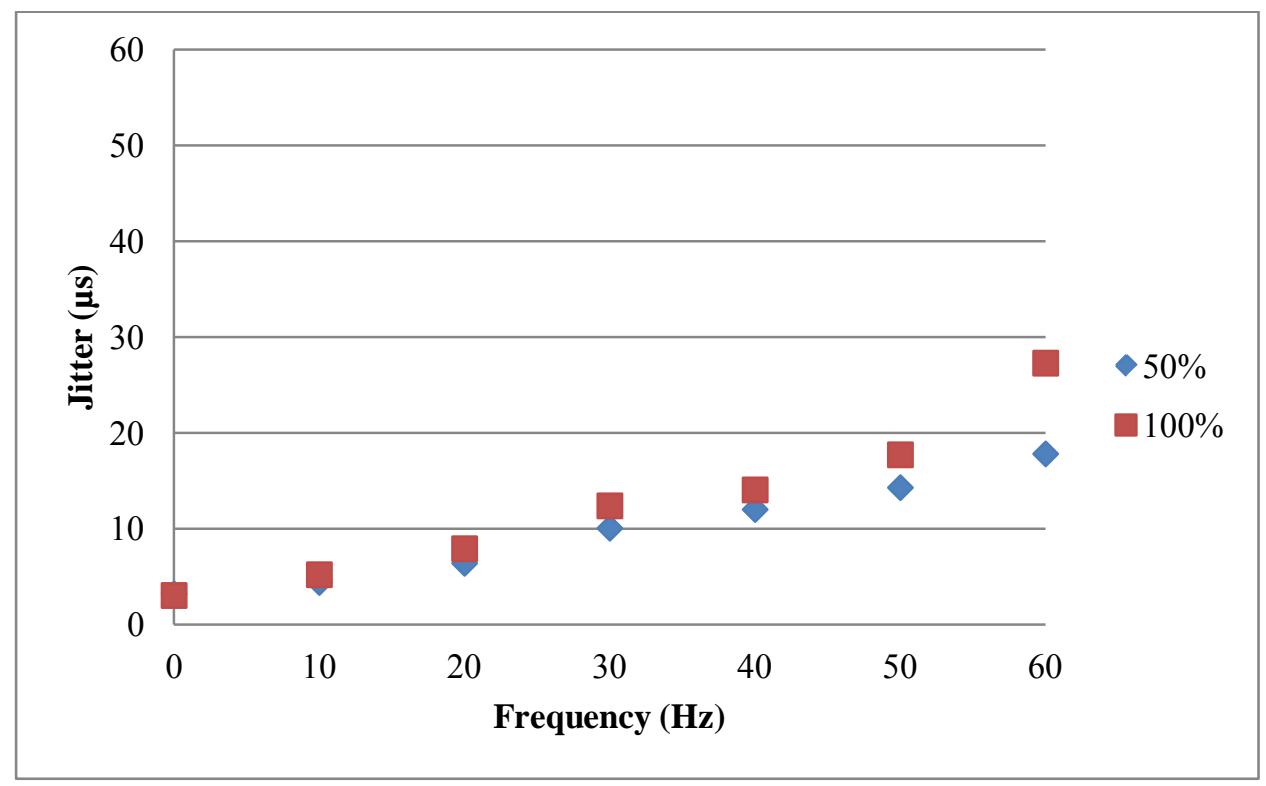

Figure 64: Average jitter as a function of frequency for the 90 degree mounting orientation with $50 \%$ and $100 \%$ acceleration

As previously shown in Figure 44 and shown in Figure 64 above, the measured jitter for all of the $50 \%$ acceleration setpoints are lower in value than the $100 \%$ acceleration setpoints. This indicates a dependence upon acceleration. Not that the physical properties of the optics have acceleration dependence, but rather that the chassis responds in a significant manner to the induced vibrations. This is because a longer chassis yields a larger rotational moment about the mounting point, and non-uniform weight distributions cause inconsistent local accelerations across the chassis of the LSP.

Figure 65 shows the average jitter as a function of frequency for all of the mounting orientations and for the $100 \%$ acceleration setpoint. For each mounting orientation, the average 
jitter increases as a function of increasing frequency. The average measured jitter was highest for the 0 degree mounting orientation and was lowest for the 45 degree orientation. Using Equation 5-5, the maximum change in CA that may occur on a per cycle basis for the 0 degree mounting orientation is 2.3 degrees for the $60 \mathrm{~Hz}$ setpoint. This is compared to the average maximum change in CA for the 45 degree mounting orientation of 0.7 degrees for the $60 \mathrm{~Hz}$ setpoint.

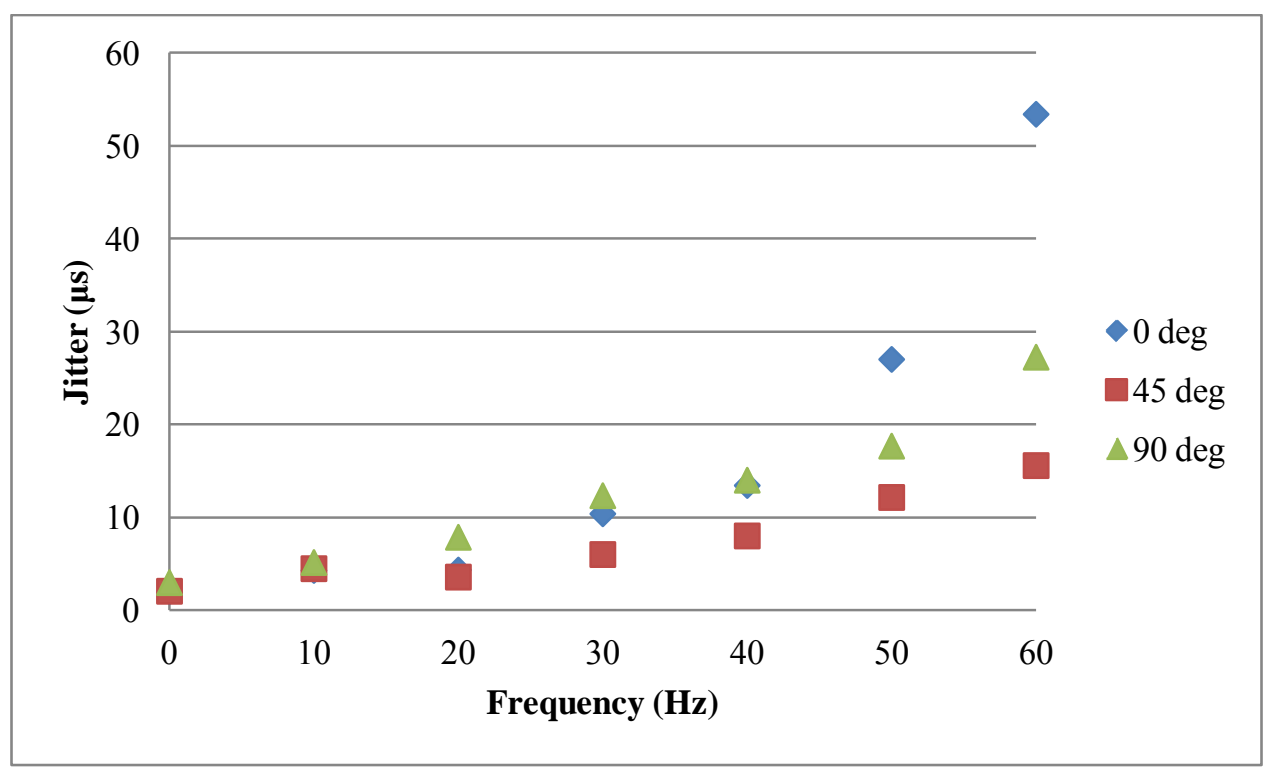

Figure 65: Average jitter as a function of frequency for the 0,45 and 90 degree mounting orientations with $100 \%$ acceleration

In order to analyze the maximum jitter for the most influential temperatures at each mounting angle, Figure 66 shows the jitter as a function of vibrational frequency. Note that the scale shown for the jitter axis has changed. For each mounting orientation, the jitter increases with increasing vibrational frequency which is to be expected. The 0 degree mounting orientation was found to have the highest amount of jitter out of all the mounting orientations, and the 90 degree mounting orientation was found to have the least amount of jitter in the highest frequency setpoint. The maximum change in CA for the 0,45 and 90 degree mounting orientations at $60 \mathrm{~Hz}$ was calculated to be \pm 4.6 degrees, \pm 1.2 degrees and \pm 3.3 degrees, respectively. 


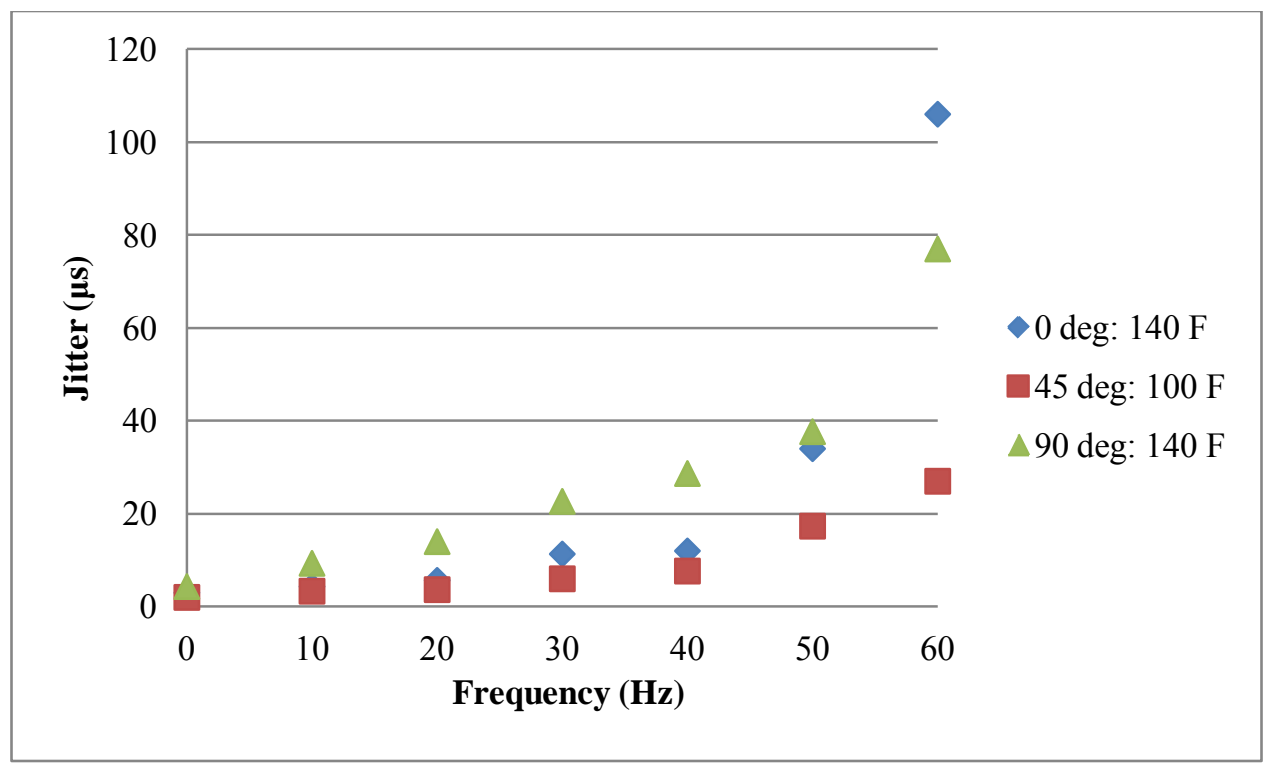

Figure 66: Maximum jitter as a function of frequency for the 0, 45 and 90 degree mounting orientations with $100 \%$ acceleration for the most influencing temperatures

It follows logic that the most jitter would be present at the highest temperatures, highest vibrational frequencies and in the 0 degree mounting orientation. For this orientation, the resulting jitter would be the highest because the laser is cantilevered in an orthogonal direction to the vibrational axis. This was found to be true. Again, it is shown that for the 45 degree mounting orientation the least amount of jitter is present compared to the other mounting angles. What is surprising about this result though is that the point of maximum jitter occurs at $100{ }^{\circ} \mathrm{F}$, where the point of maximum jitter for the other mounting orientations occurs at $140{ }^{\circ} \mathrm{F}$. The 0 degree and 90 degree mounting orientations follow the correction factor data and literature, where as the 45 degree mounting orientation does not.

\subsubsection{Energy}

As previously discussed in Section 5.1, the energy output was shown to increase with respect to increasing pulse frequency. This relationship was confirmed in the literature and has been further confirmed here as well [34]. Recall that the pulse frequency was applied at exactly one half the rate of the vibrational frequency. Figure 67 shows the relationship between average output energy and vibrational frequency for the 0,45 and 90 degree mounting orientations for the $50 \%$ acceleration setpoints. All mounting orientations were found to show an increase in output 
energy with respect to increasing frequency. The highest energy output was found to come from the 0 degree mounting orientation data.

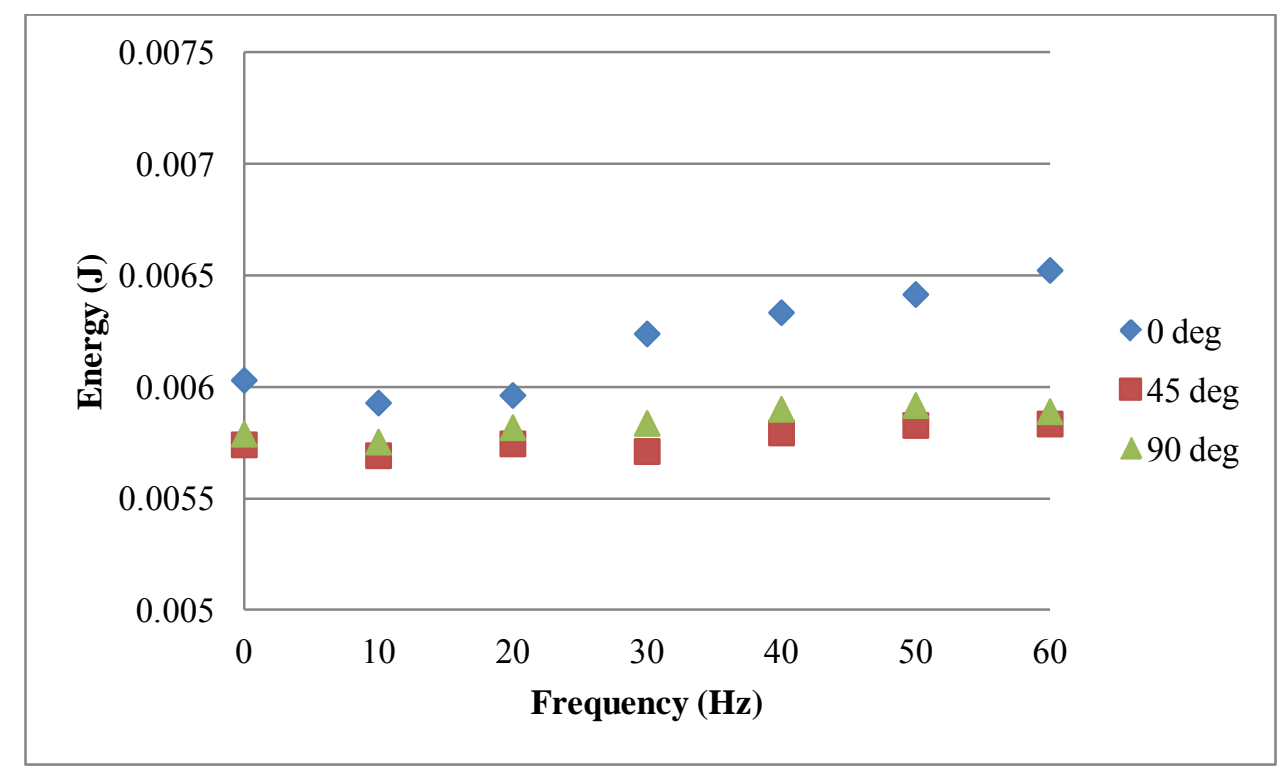

Figure 67: Average output energy as a function of frequency for the 0,45 and 90 degree mounting orientations with $50 \%$ acceleration

An explanation for the increasing output energy as a function of increasing frequency for the 0 degree mounting orientation deals with the orientation itself. With the 0 degree orientation, the LSP is vibrated as a cantilever in an orientation orthogonal to the axis of vibration. As previously discussed, when shaken in this orientation the LSP displayed a central node, which acted as an axis for gyration. In a stationary state, the pump energy is steered into the ignition laser cavity and focused down to a point within the gain medium. When shaken in this orientation, the focal point for the pump energy sweeps the excitation mechanism allowing for a larger area within the gain medium to be pumped. This theory would not only produce slightly larger output energy, but it would also increase the focal area size of the ignition laser decreasing the intensity of ignition. Since the axis of vibration occurred in a single direction, the excitation area would become taller and more ovular than the excitation region under stationary conditions. An illustration of this theory is shown in Figure 68. As shown $d_{2}$ and $E_{2}$ are both larger than $d_{1}$ and $\mathrm{E}_{1}$. As vibrational frequency is increased, the sweeping motion becomes faster and wider. 

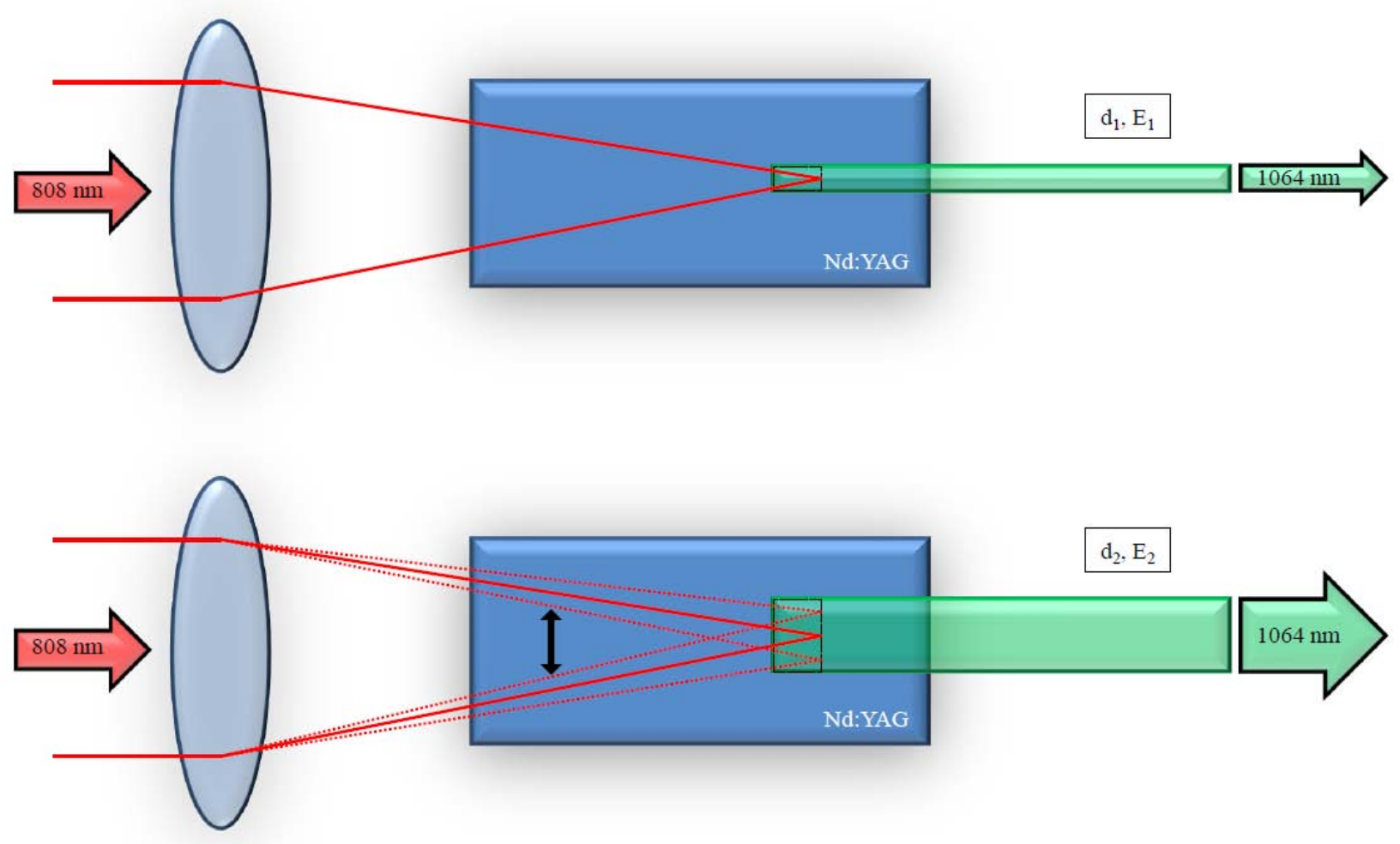

Figure 68: Laser vibration theory for the 0 degree mounting orientation

The focal area was assumed to remain constant throughout the duration of this testing regardless of the mounting orientation. This assumption was made due to lack of proper equipment necessary to measure the focal area to a sufficient resolution. The representation shown in Figure 68 is an exaggerated depiction of the actual change in focal area. It is hypothesized that the focal area may have changed somewhere on the magnitude of $10^{-8} \mathrm{~cm}^{2}$, which is much less than the assumed constant value used for focal area, which lies on the order of $10^{-6} \mathrm{~cm}^{2}$.

The pumping strategy used in this design is an end pumped strategy. This means that the pump energy enters the gain media on the end of the crystal which is oriented coaxially with the global axis of the laser. Figure 69 shows the excitation regions of an end pumped Nd:YAG crystal. As shown, the focal point of the PCX lens is focused directly in the center of the crystal rod. The highest excited region of the crystal occurs at the focal point of this lens since this is the point where the focal intensity is the highest. Under vibrational influences, as shown in Figure 68, the distribution of highly excitation regions would be more spread out in the crystal. 


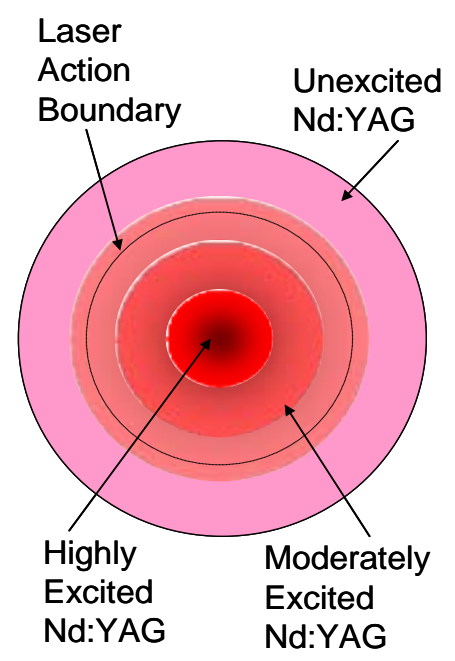

Figure 69: Excitation regions of the Nd:YAG crystal using an end pumped strategy [21]

As previously discussed, energy output increases with increasing pump pulse frequency. Figure 70 shows the output energy as a function of vibrational frequency for the 45 degree mounting orientation at $50 \%$ of the maximum acceleration. Here, a constant trend in output energy as a function of increasing frequency is shown. This is inconsistent with the literature, the correction factor investigation shown in Figure 31 and the other mounting orientations.

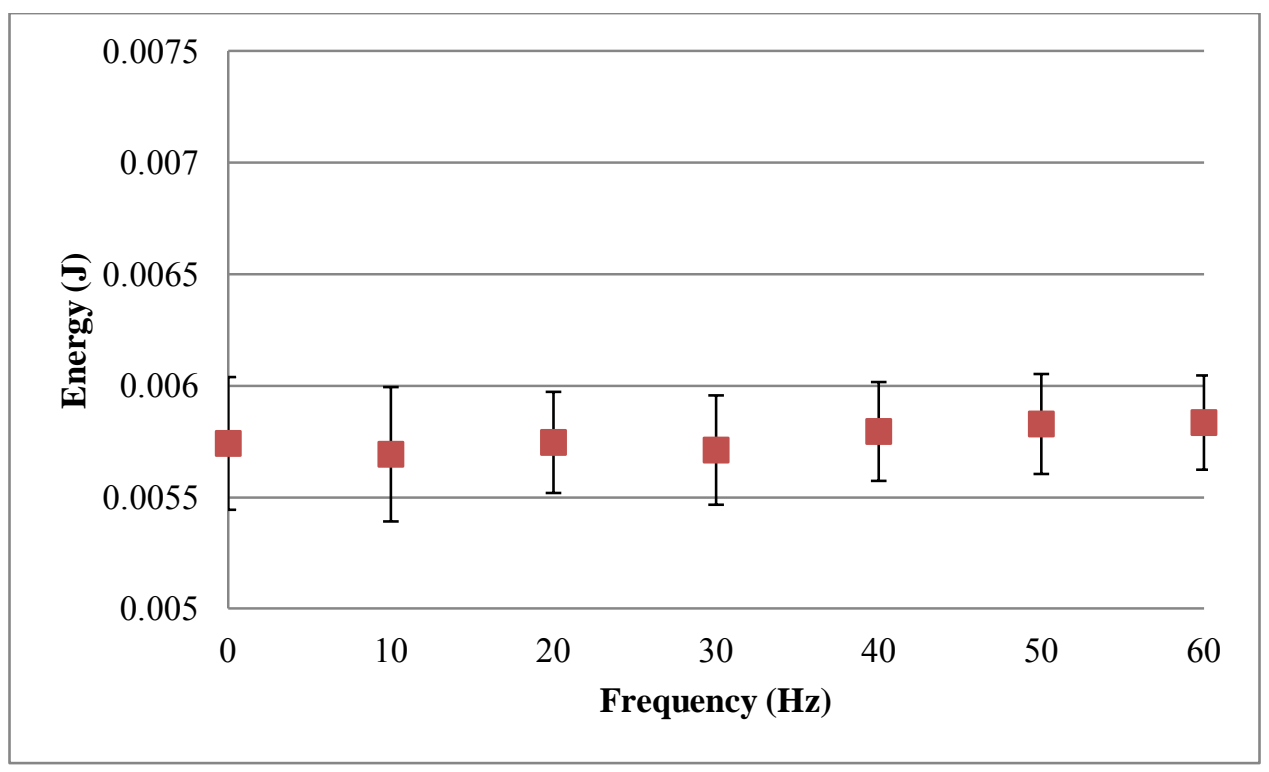

Figure 70: Average output energy as a function of frequency for the 45 degree mounting orientation with $50 \%$ acceleration 
Figure 71 shows the relationship between the average output energy and frequency for the 90 degree mounting orientation for both acceleration setpoints. As shown, the 50\% and $100 \%$ setpoints nearly overlap at every vibrational frequency setpoint. This indicates that there exists very little energy output dependence upon acceleration.

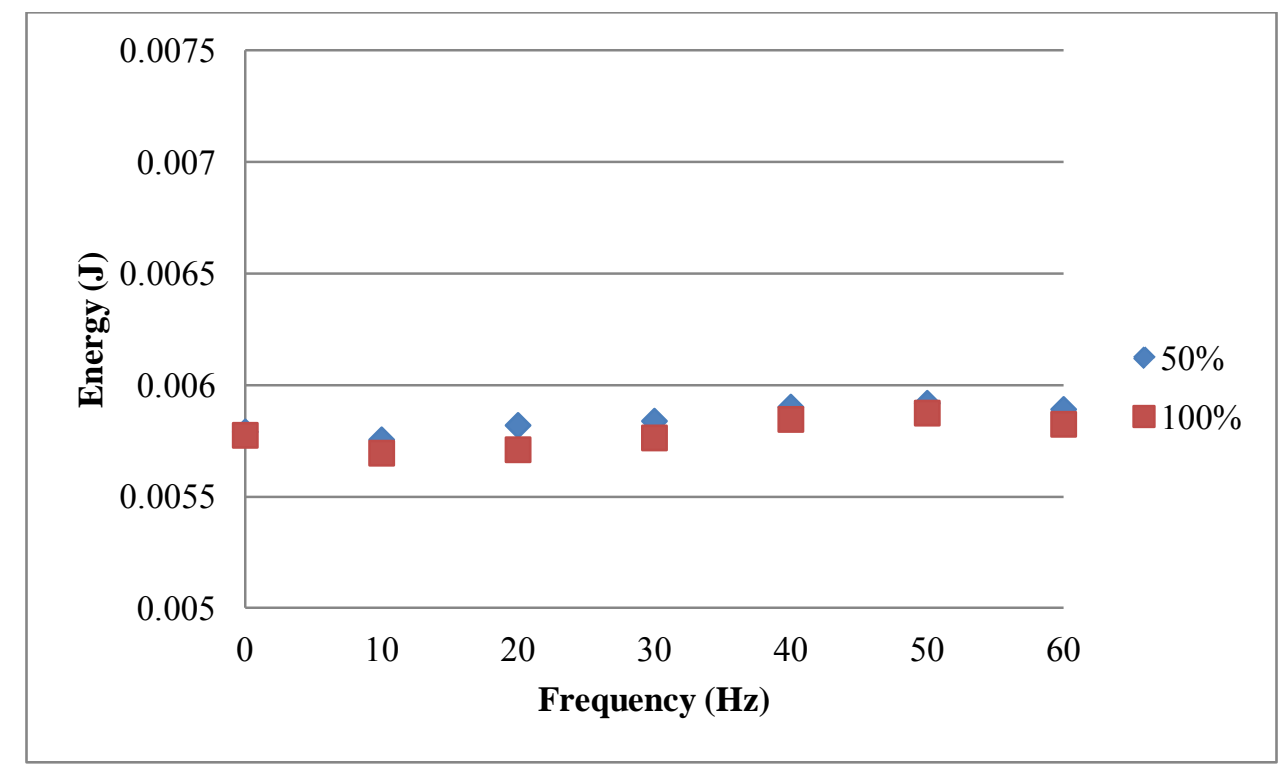

Figure 71: Average output energy as a function of frequency for the 90 degree mounting orientation with $50 \%$ and $100 \%$ acceleration

\subsubsection{Focal Intensity}

When averaged over the temperature band, data showing the focal intensity as a function of frequency is produced for the 0,45 and 90 degree mounting orientations at the $50 \%$ acceleration setpoints as shown in Figure 72. The trend of the plot is similar to the trend in Figure 67 meaning that the output energy is largely the controlling factor in determining focal intensity. This is primarily due to the small amount of change in the output pulse width as previously shown in Figure 55, and the fact that the focal area was assumed constant across the array of test matrices. As shown below, each of the focal intensity data points, when averaged over the thermal setpoints, is above the breakdown threshold of $100 \mathrm{GW} / \mathrm{cm}^{2}$. This means that at every thermal setpoint conducted in this testing, if a focusing lens were to be placed in front of the output of the LSP, it is possible to generate a plasma spark at atmospheric pressures. 


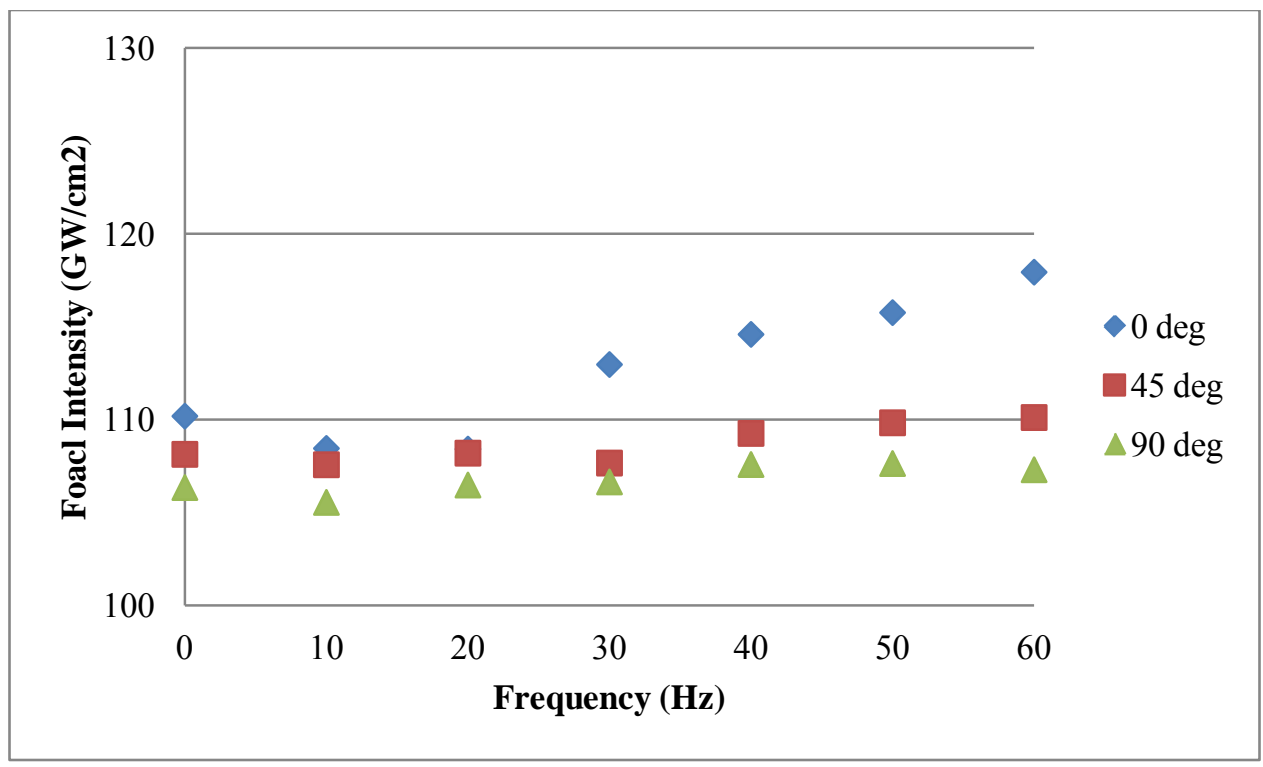

Figure 72: Focal intensity as a function of frequency for the 0,45 and 90 degree mounting orientations with $50 \%$ acceleration 


\section{CONCLUSIONS AND RECOMMENDATIONS}

\subsection{Thermal Influence}

The material compositions of the components comprising the structure of the LSP are of importance. The thermally conductive material used in the construction of the LSP play a significant roll on the alignment of the optical circuit when operating over a wide range of temperatures. The thermal expansion of steel that was used to make the alignment mounts for the HR and OC causes negative effects upon the alignment of the LSP. The thermal expansion of the steel with respect to increasing temperature leads to a decrease in coupling efficiency. With a single optimized alignment at $80{ }^{\circ} \mathrm{F}$, the LSP ceased to output $1064 \mathrm{~nm}$ when the temperature reached steady state at $140{ }^{\circ} \mathrm{F}$. This may be overcome with a re-alignment procedure at elevated temperatures. Since the purpose of this study was to determine the effects of temperature and vibration of the prototype spark plug, an optimization alignment was conducted when necessary.

In an ideal setup, the structure of the laser plug would be perfectly symmetrical about the optical axis, and be manufactured from a material with a low coefficient of thermal expansion. Symmetry about the optical axis is of importance because if any thermal expansion were to occur

over a range of temperatures, the resulting thermal lensing may be kept to a minimum. Although there is nothing that can be done to alter the thermal expansion or other material properties of a particular optical component, the material that is used to mount the optical component may be chosen at the freedom of the designer. The ideal material to be used for the construction of the LSP would be one with a small coefficient of thermal expansion.

An alternative means of accounting for the varying temperature would be to control the operating temperature of the laser to a fixed setpoint. This temperature control may come in the form of heating or cooling the laser depending upon the optimal output characteristics chosen by the designer. Similar to the method of controlling the laser to a setpoint temperature as performed here in this testing, the consistency of the output will be high if all of the components comprising the construction of the laser are kept at a uniform and steady state temperature.

If a thermal map of the LSP's response under the influence of temperature were to be incorporated into the engine's ignition management system, a strategy for accurately controlling 
the ignition timing as a function of the LSP's temperature may be realized. However if the LSP were to have a cooling/heating system implemented onto it which controlled the temperature to a relatively steady state, the ignition delay would be fixed and the LI system would be able to operate independently of the engine temperature. The effects of thermal expansion and thermal lensing would also be kept minimal if the spacing between the ignition laser's optical components were minimized thereby minimizing the probability for alignment variation. This is reasoning for a monolithic designed LSP which would not require adjustable optical mounts.

\subsection{Size and Weight Distribution}

As previously discussed in Section 5.2.5, the physical size and heterogeneous weight distributions leads to inconsistent output characteristics when mounted in particular orientations. Variations in acceleration and vibrational frequency have been found to significantly impact the operation of the laser. In order for a laser plug to be deemed a viable replacement for the conventional electrode-based spark plug, a direct drop in substitute which is independent of mounting orientation is desired. This desire alone requires a reduction in size, however when considering the increased optical stability that can be achieved from a shorter path length when under the influence of engine perturbations, a reduction in size is a must. With a smaller design, the laser plug could be more rigid and have a more uniform weight distribution. This is the key to minimizing the effects that vibrational perturbations have and reduce the probability for alignment variation over a range of temperatures.

In an ideal case, the LSP would be approximately the size of a conventional spark plug and couple with the engine directly into the existing spark plug port. A monolithic design would allow for a significant reduction in size, reduction in weight, removal of unnecessary hardware and have an intracavity alignment that is permanently fixed. By fusing the optical components together effectively reduces the degrees of freedom that are susceptible to variation. This strategy is flawed in this sense that if a single component requires replacement, the entire set of optics would have to be replaced. However when considering the design to be manufactured on a mass scale, the potential for a significant reduction in cost exists. The cost analysis is a critical aspect at this point in the design process which needs to be quantified. 


\subsection{Pumping}

The pumping strategy is a critical aspect in the operation of a laser regardless of its application. In the current design, the need for the pumping mechanism to be remotely located exists. This is not ideal for industrial applications. Although this current strategy may be applied to stationary engines, a pump source that is directly coupled the ignition laser would widen the range of applications and simplify the overall integration.

A fiber coupled pump source works well for the spatially complex optical routing necessary for generating a plasma spark within the engine's combustion chamber, however it is fundamentally flawed. As previously discussed in Section 2.5, fiber optic cables are extremely susceptible to bending and vibrational losses. Bending leads to energy loss through the fiber and undesired mode coupling which may lead to damage. Vibration amplifies these losses and ultimately affects not only the energy output but the output beam profile as well. Recall that an ideal pump beam would be perfectly Gaussian and couple with the gain medium to cause a high rate of population inversion.

As previously discussed in Section 3.4.1.3, VCSEL technology has developed to the point where it can efficiently act as a pumping source for end-pumped passively q-switched Nd:YAG lasers [47, 48, 49]. This pumping mechanism is presently considered to be the best means of pumping a laser with unique spatial requirements in harsh conditions. A pump source such as this would eliminate the fiber optic cable and broaden the range of applicable engine industries. The small size, reliability and high output energy would be ideal to act as a pump source for a LSP.

\subsection{Engine Operation}

It has been shown that the output characteristics of the LSP are sensitive to vibrational and thermal perturbations. The parameter that was most strongly affected by these perturbations was the time at which $1064 \mathrm{~nm}$ emission occurred. This has been discussed in previous chapters relating to the q-switch delay and the effective jitter. Spark timing in an IC engine is critical for several reasons relating to combustion efficiency, resulting emissions and overall engine operation. In this system, output timing variability exists even in the least extreme setpoints. Therefore, it is necessary to consider the probability of output occurring at a desired time. Figure 
73 shows the probability of output occurring at a desired time from pulse to pulse as a function of temperature and vibration.

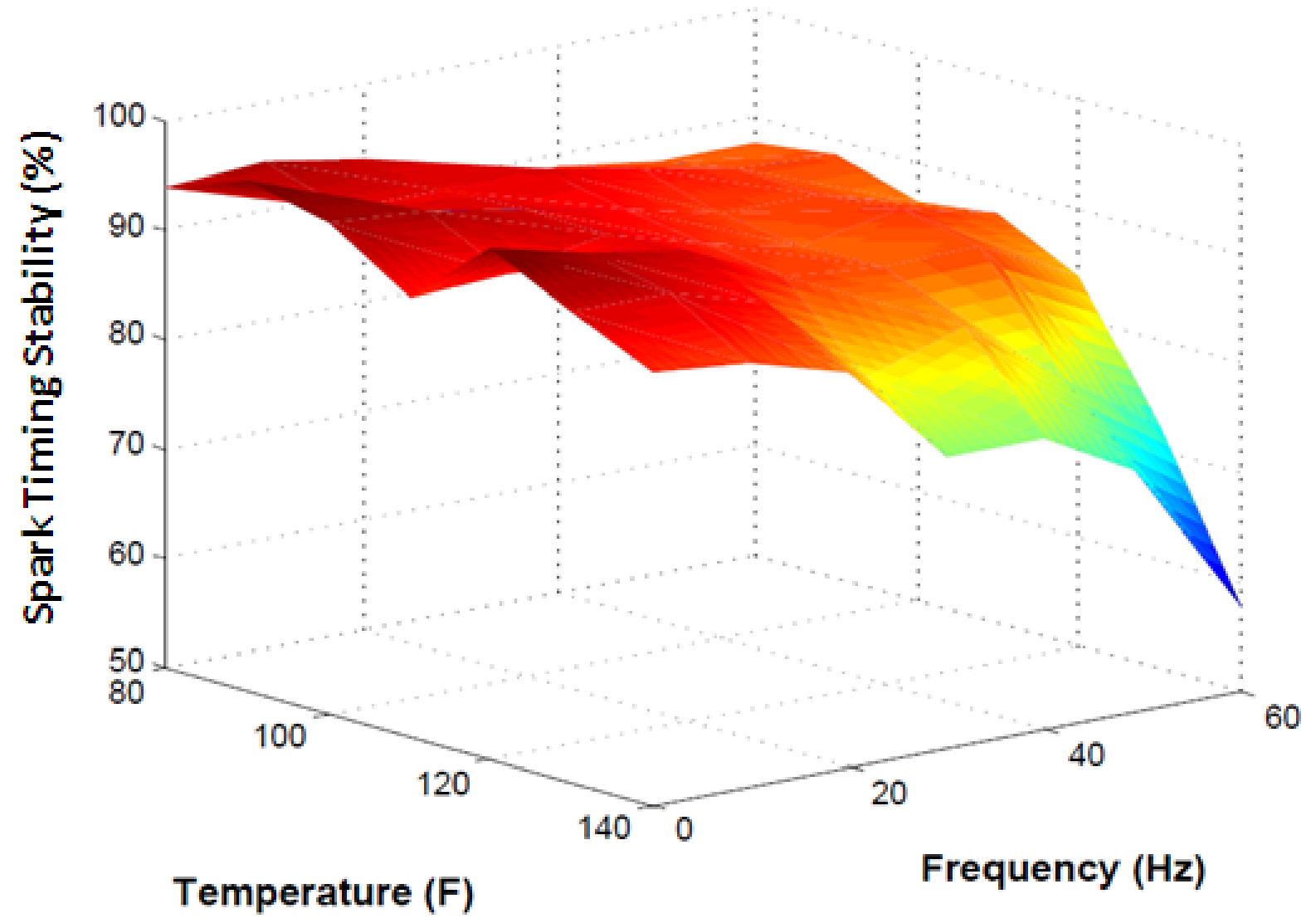

Figure 73: Q-switch timing stability as a function of temperature and frequency in the 90 degree mounting orientation with $100 \%$ acceleration

As shown, the chance of obtaining output at a desired time decreases as temperature and vibration increase. This representation was calculated based on the measured temporal deviation from the average q-switch delay. Considering that there is a narrow window in which ideal spark timing resides, the undesired pulse-to-pulse variation from this LI system presents a challenge for IC engine applications 


\subsection{Recommendation for Future Work}

\subsubsection{Ricardo Proteus}

The current LSP should be recoupled to the Ricardo Proteus engine as previously shown in Figure 14, and operated with an improved ignition strategy. Given the research and data presented here, a numerical model may be developed for an ignition strategy to optimize the ignition timing and output power based on the independent variables. This ignition strategy should initially be used with the engine fueled by natural gas so the performance metrics may be directly compared to previous research performed by McIntyre et al.

\subsubsection{Beta Prototype}

Given the data collected here and the research performed by others, the requirements for developing a laser to act as a direct replacement for the conventional spark plug are clear. Working towards bringing a product to market, a second/beta prototype should be developed. The optical components comprising the laser cavity should be fused together to generate a monolithic cavity design. Since this will still be a prototype where additional thermal and oscillatory testing is necessary to fully characterize the response of the plug, the pump steering optics should remain as separate entities from the cavity. This should be done for adjustability reasons since thermal effects are still expected to be an issue, and because of the limitations of the pump source.

Given the currently high price of VCSELs, it is recommended that the pumping source for the second prototype remain a fiber coupled diode laser that is remotely located from the perturbation source. This will allow valuable research to proceed in such a manner as to bring a product to limited market, and allow this research to continue in a financially effective manner. Integrity, long term reliability and long term durability during engine operation remain to be significant hurdles in the development of this technology.

Oscillatory and thermal testing should be performed on the beta prototype as well, since the response of the output characteristics will be different than presented here. Vibrational testing and characterization may be improved upon by simultaneously shaking the laser the three orthogonal directions as to more accurately mimic the operation of an engine. Perhaps the best 
and easiest way to do this would be to mount the laser directly on an operating engine and measure its output characteristics. This may also be the best way to investigate the thermal effects as well, however if the laser were to be mounted on an engine, the operational temperature would be difficult to vary over a wide range. To generate an accurate thermal profile, the laser should be tested in a thermally controlled enclosure and allowed sufficient amount of time to reach an equilibrium temperature. This strategy for heating the LSP in an enclosure is better than wrapping it in heat tape because in an enclosure, the heat transfer is purely convective which would more accurately simulate engine conditions and the LSP would be heated more uniformly. Recall from Section 3.6.6 that the non uniform temperature distribution is a source of error in this experimentation.

\subsubsection{The Ideal Laser Spark Plug}

It may be beneficial at this point to describe the characteristics of what is presently considered to be an ideal LSP. All of the optics would be constructed into a monolithic design thereby fixing the alignment, reducing the weight, reducing the sources for error and obtaining a compact device. The laser would be pumped with a VCSEL, or similar compact pump source, so that the plug may be packaged as a single unit. The housing for the optics and pump source would be rigid as to limit the vibration and acceleration influences. The housing should be constructed in entirety from the same material with a low coefficient of thermal expansion. An acceptable design for the chassis would be an injection molded ceramic that could be completely sealed to the point where it could permanently hold a vacuum at a sufficient level. Sealing the laser with its own atmosphere would restrict ambient particles from influencing its operation. The HR and OC coatings would be applied to a face of the gain medium and q-switch respectively by means of physical vapor deposition to a thickness of a few hundred angstroms. The gain medium and q-switch would be fused together and may be assembled in a manner similar to the schematic shown in Figure 74. The size and weight of the LSP would be approximately the size and weight of a conventional spark plug, and it would be a direct drop in replacement as well. 

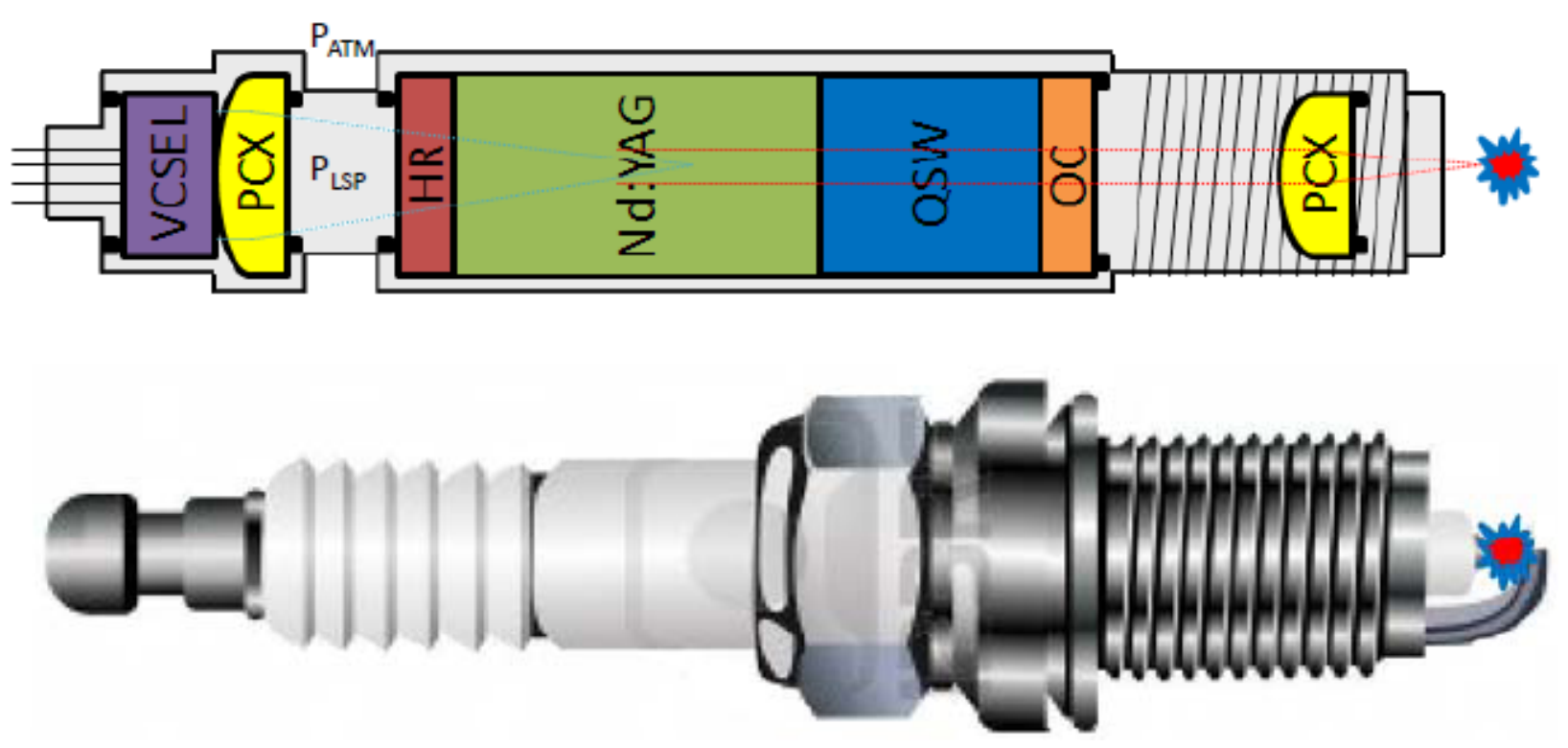

Figure 74: Construction of an ideal laser spark plug

\subsubsection{Future Development}

As the progression of LI technology advances, there exists a need for future development in several aspects for this application. Limiting the future development to the anticipated work to be conducted by CAFEE, there are several more steps to be taken that will directly fall in line with CAFEEs areas of expertise. There exists a gap in the literature for characterizing alternative fuels and fuel blends with the use of a laser ignition source. The benefits of using a laser to initiate combustion have yet to be investigated for several fuels. Investigating their combustion efficiency and characterizing the resulting emissions is necessary to aid in further justifying the implementation of a LI source. A multiple cylinder operation strategy continues to be a highly debated topic in the LI industry. The strategy differs between laboratories and a finite method for achieving this strategy has yet to be developed. Fundamental LI research on the multiple cylinder scale is critical for justifying mass implementation to engine manufactures. In short, there remains a significant amount of research that must be conducted prior to implementation. 


\section{REFERENCES}

[1] F. E. Chen, Introduction to Plasma Physics, New York: Plenum Press, 1974.

[2] S. Richardson, M. McMillian, S. Woodruff and D. McIntyre, "Misfire, Knock and NOx Mapping of a Laser Spark Ignited Single Cylinder Lean Burn Natural Gas Engine," SAE Technical Paper: 2004-01-1853, June 8-10, 2004.

[3] J. Dale, P. Smy and R. Clements, "Laser Ignited Internal Combustion Engine- An Experimental Study," SAE Technical Paper 780329, no. 10.4271/780329, 1978.

[4] A. Yalin and B. Willson, "Fundamental Studies of Ignition Process in Large Natural Gas Engines Using Laser Spark Ignition," Colorado State University Engines and Energy Conversion Laboratory, Fort Collins, 2008.

[5] B. Bihari, S. Gupta, R. Sekar, J. Gingrich and J. Smith, "Development of an Advanced Laser Ignition System for Stationary Natural Gas Engines," in Proceedings of ASME ICE 2005, Ottawa, 2005.

[6] S. Joshi, A. Reynolds, B. Willson and A. P. Yalin, "Design and Bench-Top Testing of Multiplexed Fiber Delivered Laser Ignition System for Natural Gas Engines," in Proceedings of ICEF 2007: AMSE Internal Combustion Engine Division 2007 Fall Technical Conference, Charleston, South Carolina, 2007, ICEF2007-1617.

[7] A. P. Yalin, A. R. Reynolds, S. Joshi, M. W. Defoort, B. Willson, Y. Matsuura and M. Miyagi, "Development of a Fiber Delivered Laser Ignition System for Natural Gas Engines," in Proceedings of ICES2006: ASME Internal Combustion Engine Division 2006 Spring Technical Conference, Aachen, Germany, 2006, ICEF2005-1325.

[8] A. P. Yalin, S. Joshi, M. DeFoort and B. Willson, "Towards Multiplexed Fiber Delivered Laser Ignition for Natural Gas Engines," Journal of Engineering for Gas Turbines and Power, vol. 130, 2008.

[9] A. P. Yalin, M. W. Defoort, S. Joshi, D. Olsen and B. Willson, "Laser Ignition of Natural Gas Engines using Fiber Delivery," in Proceedings of ICEF2005: ASME Internal Combustion Engine Division 2005 Fall Technical Conference, Ottawa, Canada, 2005, ICEF2005-1336.

[10] M. Weinrotter, H. Kopecek and E. Wintner, "Laser Ignition of Engines," Laser Physics, vol. 15, no. 7, pp. 947-953, 2005. 
[11] M. Tsunekane, T. Inohara, K. Kanehara and T. Taira, Micro-Solid-State Laser for Ignition of Automobile Engines, Institute for Molecular Science, Nippon Soken, Inc. Japan: InTech, 2010, pp. 195-212.

[12] M. McMillian, S. Richardson, S. Woodruff and D. McIntyre, "Laser-Spark Ignition Testing in a Natural Gas-Fueled Single-Cylinder Engine," SAE Technical Paper: 2004-01-0980, March 8-11, 2004.

[13] J. Tauer, H. Kofler and E. Wintner, "Laser-initiated ignition," Laser \& Photonics, vol. 4, no. 1, pp. 99-122, 2010.

[14] M. Lackner, H. Kofler, H. Ranner, F. Winter, J. Klausner and G. Herdin, "The Optical Spark Plug: Window-related issues," Proc. Eur. Combustion Mtg, Combustion Inst., Vienna University of Technology, Vienna, Austria, 2005.

[15] P. Tran, "Laser-induced spark ignition fundamental and applications," Optics and Lasers in Engineering, vol. 44, pp. 351-397, 2006.

[16] R. Tyagi, V. Rampal and G. Bhar, "Laser-Induced Ionization of Air in the Presence of Preionization," IEEE Journal of Quantum Electronics, vol. 24, no. 4, pp. 683-686, 1988.

[17] R. Dewhurst, "Comparative Data on Molecular Gas Breakdown Thresholds in High LaserRadiation Fields," Journal of Applied Physics, vol. 11, pp. 191-195, 1978.

[18] F. Silsbee, "Report No. 202- The Sparking Voltage of Spark Plugs," Bureau of StandardsReport National Advisory Committee for Areonautics (NACA), 1925.

[19] P. D. Ronney, "Laser versus Conventional Ignition of Flames," Optical Engineering, vol. 33, no. 2, pp. 510-521, 1994.

[20] E. Schwartz, J. Tauer, H. Kofler and E. Wintner, "NS-Laser-Ignited Ignition of Engines," Journal of the Technical University of Sofia, vol. 14, no. 1, 2009.

[21] D. L. McIntyre, "A Laser Spark Plug Ignition System for a Stationary Lean-Burn Natural Gas Reciprocating Engine," Ph.D. Dissertation: West Virginia University, Morgantown WV, 2007.

[22] G. Yoder, "Fiber Optic Cables for Laser Ignition Applications," Journal of Undergraduate Research and Scholarly Excellence, vol. 2, no. 2, pp. 17-20, 2011.

[23] P. Tran and C. White, "Optical Characterization of the Laser Induced Spark in Air," Optical Diagnostics in Engineering, vol. 1, no. 5, pp. 12-26, 2001 ISSN: 1364. 
[24] W. Koechner and M. Bass, Solid State Lasers: A Graduate Text, New York, Springer, 2003.

[25] B. Hunter and K. Leong, "Understanding High-Power Fiber-Optic Laser Beam Delivery," Journal of Laser Applications, vol. 1, pp. 3-11, 1996.

[26] C. Langrock, D. S. Hum, E. Diamanti and M. Charbonneau-Lefort, "Flash Lamp Pumped Quanta Ray Nd:YAG Laser Experiment," IEEE Journal on Selected Topics in Quantum Electronics, vol. xx, no. y, 2002.

[27] A. Siegman, Lasers, Sausalito CA, University Science Books, 1986.

[28] "Applied Laser Safety," Laser Professionals Inc, Las Vegas, NV 89109, 2005.

[29] D. L. McIntyre and S. D. Woodruff, "Laser Spark Plug Numerical Design Process with Experimental Validation," in 2011 Fall Technical Conference of the ASME Internal Combustion Engine Division, ICES2011-60015, Morgantown, West Virginia, Oct 3-6, 2011.

[30] A. L. Shah, S. N. Datta, A. R. Singh, R. Malhotra, S. Pal and A. Kumar, "Investigating Transient Thermal Effects in Flash-Lamp-Pumped Heat Capacity Nd:YAG Rod Laser," IEEE Journal of Quantum Electronics, vol. 44, no. 11, 2008.

[31] B. Dane, L. Flath, M. Rotter, S. Fochs and J. Brase, "Army Solid State Laser Program: Design, Operation, and Mission Analysis for a Heat-Capacity Laser," Solid State and Diode Laser Technology Review. Albuquerque, New Mexico, no. UCRL-JC-143964, 2001.

[32] A. M. Bonnefois, M. Gilbert, P. Thro and J. Weulersse, "Thermal Lensing and Spherical Aberration in High-Power Transversally Pumped Laser Rods," Optics Communication, vol. 259, pp. 223-235, 2006.

[33] M. Tsunekane and T. Taira, "Temperature and Polarization Dependences of Cr:YAG Transmission for Passive Q-switching," in Lasers and Electro-Optics 2009 Conference on Quantum electronics and Laser Science, Baltimore, MD , 2009.

[34] M. Bass, L. S. Weichman, S. Vigil and B. K. Brickeen, "The Temperature Dependence of Nd3+ Doped Solid-State Lasers," IEEE Journal of Quantum Electronics, vol. 39, no. 6, 2003.

[35] S. Hurand, L. Chauny, H. El-Rabii, S. Joshi and A. Yalin, "Mode Coupling and Output Beam Quality of 100-400 Micrometer Core Silica Fibers," Journal of Applied Optics, vol. 4, pp. 492-499, 2009.

[36] D. Gloge and E. Marcatili, "Multimode Theory of Graded Core Fibers," The Bell System 
Technical Journal, vol. 52, no. 9, pp. 1563-1577, 1973.

[37] S. Joshi, A. Yalin and A. Galvanauskas, "Use of Hollow Core Fibers, Fiber Laser, and Photonic Crystal Fibers for Spark Delivery and Laser Ignition in Gases," Journal of Applied Optics, vol. 46, no. 19, pp. 4057-4064, 2007.

[38] A. Stakhiv, R. Gilber, H. Kopecek, A. Zheltikov and E. Wintner, "Laser Ignition of Engines via Optical Fibers?," Journal of Laser Physics, vol. 14, no. 5, pp. 738-747, 2003.

[39] S. Joshi and A. P. Yalin, "Fiber Delivery of High Power Nanosecond Pulses for Ignition in Aerospace Engines," in Avionics, Fiber Optics and Photonics Technology Conference, San Diego, CA, 2011.

[40] S. Woodruff, D. McIntyre and L. Marshall, "Laser Car Ignition Dream Sparks Multiple Approaches," Photonics Spectra, pp. 39-43, September 2012.

[41] B\&K, "Technical Document Users Manual- PM Vibration Exciter Type 4808," Bruel \& Kjaer, June 2006.

[42] C. H. Robotics, "Data Sheet- UM6 Ultra Miniature Orientation Sensor," August 2012.

[43] "Code of Federal Regulations: 40 CFR 1065.315," U.S. Government, 732 North Capitol Street, NW, Washington, DC 20401-0001, 2007.

[44] "American Society of Testing and Materials (ASTM) International: Standard Guide for Thermocouple Verification," Designation: E28461, West Conshohocken, PA, November 2011.

[45] "National Instruments: NI 6023E/6024E/6025E Family Specifications," 370719C-01, 2005.

[46] R. Morgan, "Technology: Introduction to Vertical-Cavity Surface-Emitting Diode Laser (VCSEL) Technology," Princeton Optronics, Mercerville, NJ, 2010.

[47] R. V. Leeuwen, "High-Power Vertical-Cavity Surface-Emitting Lasers for Diode Pumped Solid-State Lasers," Princeton Optronics, Mercerville, NJ, 2012.

[48] J. F. Seurin, "High-Power Vertical-Cavity Surface-Emitting Lasers for Solid-State Laser Pumping," Princeton Optronics, Mercerville, NJ, 2012.

[49] L. Goldberg, C. McIntosh and B. Cole, "VCSEL End-Pumped Passively Q-switched Nd:YAG Laser with Adjustable Pulse Energy," Optics Express 4261, vol. 19, no. 5, February 282011. 
[50] "National Instruments: SCB-68 User Manual for Advanced Functions," National Instruments Corporation, 11500 North Mopac Expressway Austin, Texas, March 2009.

[51] "Fluke Corporation: Fluke 714 RTD Calibrator Instructions," 1997-2004 Fluke Corporation, August 1997.

[52] Omega, Interviewee, Personal Phone Conference. [Interview]. January 2013.

[53] "North Carolina University: Laser Safety," Environmental Health and Public Safety Center, Raleigh, NC 27607.

[54] "American National Standard Institute (ANSI): American National Standard for Safe Use of Lasers: ANSI Z136.1-2000," New York, New York 10036, 1996.

[55] Ophir, "PE50BB-DIF-V2 Detector Head Manual," Laser Measurement Group, 2009.

[56] ReedCollege, Laser Safety Handbook, Portland, OR, January 2005.

[57] R. Mahajan, A. Shah, S. Pal and A. Kumar, "Analytical Study for Investigating the Behaviour of Nd-doped Glass, YAG and GGG Under the Heat Capacity Mode of Operation," Optics and Laser Technology, vol. 39, pp. 1406-1412, 2007.

[58] Y. Huang, H. Tsai and F. Chang, "Thermo-Optic Effects Affecting the High Pump Power End Pumped Solid State Lasers: Modeling and Analysis," Optics Communication, vol. 273, pp. 515-525, 2007.

[59] J. Gordon and H. Haus, "Random Walk of Coherently Amplified Solitons in Optical Fiber Transmission," Optics Letters, vol. 11, no. 10, pp. 665-667, 1986.

[60] T. Phuoc, "Laser Spark Ignition: Experimental Determination of Laser-Induced Breakdown Thresholds of Combustion Gases," Optics Communications, vol. 175, pp. 419-423, 2000.

[61] C. Bindhu, S. Harilal, M. Tillack, F. Najmabadi and A. Gaeris, "Energy Absorption and Propagation in Laser-Created Sparks," Society for Applied Spectroscopy, vol. 58, no. 6, 2004. 


\title{
Appendix-A: Advance Ignition System Functional Requirements
}

\author{
Functional Requirement for Advanced Natural Gas Recip Ignition System
}

Versıon 1.0

This spec is based on the collective input from the three engine manufacturers responsible for commercializing products based on ARES program goals. Refer to the DOE DEER website "http://www.eren.doe.gov/der/gas_fired/gas_fired.html " for information concerning the ARES program.

Ignition systems play a critical role in advancing state of the art combustion systems for improved efficiency and reduced emissions in natural gas engines. The attached specification outlines some of the critical aspects of advanced ignition. but is not meant as an all-inclusive list. While electric ignition systems are the most common system available today, future systems may or may not use this approach. However, the key ingredients of cost, reliability, and serviceability must be maintained to satisfy our broad customer base.

This list is subject to change as technology advances, and will need to be reviewed and updated at future meetings of the Advanced Ignition System Roundtable.

Our definition of the ignition system includes the entire package required to accept an Ignition signal from an external source until completion of the ignition event. Typical components today include a control module, processor, ignition distribution system, cylinder processor (coil and extender), igniter (spark plug), and limited ignition diagnostics and prognostics. Note that this ignition system requirement does not include a combustion analysis package although this would be desirable in future systems.

Gordon Gerber

Caterpillar Inc

November 06, 2002
Mark Rosswurm

Cummins Inc
Ed Reinbold

Waukesha Engine 


\begin{tabular}{|c|c|c|c|}
\hline & System Profile Element & $\begin{array}{c}\text { Continuous-Duty Power } \\
\text { Generation } \\
\text { Requirements* }\end{array}$ & Units \\
\hline \multirow[t]{4}{*}{1} & Cost (current dollars) & & \\
\hline & - First Cost (add \$1/ekw for CSA requirement) & 4.00 & $\$ / \mathrm{kWe}$ \\
\hline & - Life Cycle Cost (including system replacement at major) & 0.25 & \$/MWe-hr \\
\hline & - Repar Costs to $1^{\text {st }}$ Engine Overhaul & 0.15 & $\$ / M W_{e}-h_{r}$ \\
\hline \multirow[t]{12}{*}{2} & Performance & & \\
\hline & -Maximum ignition pressure (peak cylinder pressure) & 220 & bar \\
\hline & -Minumum aur/fuel ratio & 0.9 & $\lambda$ \\
\hline & -Maximum arr/fuel ratio (w/swirl) & 2.5 & $\lambda$ \\
\hline & -Minimum methane number (hydrogen capable) & 0 & \\
\hline & - Maximum methane number (landfill capable) & 140 & \\
\hline & - Ignition timing repeatability (non-mechanicai) & 0.08 & ¿crank \\
\hline & - Ignition timing accuracy (non-mechanical) & 0.08 & ${ }^{\circ} \mathrm{crank}$ \\
\hline & - COV (steady state, 0.5 g/bhp-hr NOx, 25 bar BMEP) & $<1.0$ & $\%$ \\
\hline & - RPM maximum (overspeed) & 125 & $\%$ of rated. \\
\hline & -RPM minimum (cranking) & 50 & $\mathrm{rpm}$ \\
\hline & - Full Load range (minımum - maximum) & $10-25$ & bar \\
\hline \multirow[t]{2}{*}{3} & Ignition System Durability & & \\
\hline & - Life to replacement for ignition module and hamess & 80,000 & $\mathrm{hrs}$. \\
\hline \multirow[t]{3}{*}{4} & Reliability $\ldots . .$. & & \\
\hline & - Ignition Systëm (continuous duty) & 6000 & MTBF (hrs) \\
\hline & - ignition System Reliability (peaking / standby) & 3000 & MTBF (hrs) \\
\hline \multirow[t]{14}{*}{5} & Environmental & & \\
\hline & - Shock (mechanical) & 20 - all three axis & G's \\
\hline & -Vibration & 10 & G's (min) \\
\hline & - On-engine Temperature Range & $-40 \mathrm{C}$ to $130 \mathrm{C}$ & ${ }^{\circ} \mathrm{C}$ \\
\hline & - Ambient Temperature & $-40 \mathrm{C}$ to $48 \mathrm{C}$ & ${ }^{\circ} \mathrm{C}$ \\
\hline & - In-cylinder temperature & 2200 & ${ }^{\circ} \mathrm{C}$ \\
\hline & - Fluids Resistance & $\begin{array}{c}\text { Ethylene glycol } \\
\text { Lube ofl }\end{array}$ & \\
\hline & - EMI (susceptibility) & $\begin{array}{c}200 \\
(30 \mathrm{kHz}-1 \mathrm{GHz})\end{array}$ & $\mathrm{V} / \mathrm{m}$ \\
\hline & - Altitude & 4000 & $\mathrm{~m}$ \\
\hline & - Humidity & 200 & grains \\
\hline & - Drop & 1 & $\mathrm{~m}$ \\
\hline & - Thermal cycles & 10,000 & cycles \\
\hline & - Water intrusion & $\begin{array}{l}\text { Withstand steam cleaning } \\
\text { and high-pressure spray }\end{array}$ & \\
\hline & - Vertical Load (Step test) & 100 & $\mathrm{~kg}$ \\
\hline \multirow[t]{3}{*}{6} & Safety \& Regulatory & CSA Class 1 Div i & Elec Power \\
\hline & & CSA Class I Div II C\&D & Petroleum \\
\hline & & UL Requirements & As developed \\
\hline \multirow[t]{5}{*}{7} & Physical & & \\
\hline & - Size (max of any one piece) & 0.015 & $M^{5}$ \\
\hline & - Weight (max of any one piece) & 10 & $\mathrm{~kg}$ \\
\hline & - Number of cylinders (scalable) & $4-20$ & cylinders \\
\hline & - Power source & 24 & VDC \\
\hline \multirow[t]{2}{*}{8} & Mounting & & \\
\hline & - System Location & All systems on engine & \\
\hline
\end{tabular}




\begin{tabular}{|c|c|c|c|}
\hline & System Profile Element & $\begin{array}{c}\text { Continuous-Duty Power } \\
\text { Generation } \\
\text { Requirements } \\
\end{array}$ & Units \\
\hline \multirow[t]{3}{*}{9} & Noise & & \\
\hline & - Rating & SAE J1113 & \\
\hline & - Rating & ISO 13766 & \\
\hline \multirow{3}{*}{10} & Mean Time to Repair & & \\
\hline & - Continuous duty & 6000 & hirs \\
\hline & - Peaking / standby duty & 3000 & hrs \\
\hline \multirow[t]{2}{*}{11} & Serviceability & & \\
\hline & - Maximum time for scheduled service & 10 & minutes / cyl \\
\hline \multirow[t]{4}{*}{12} & External Communications Interface & & \\
\hline & 1) Compatible with Engine Diagnostic Tools & & \\
\hline & 2) SAE J1939 Communications & & \\
\hline & 3) Ability to set timing via the data link & & \\
\hline \multirow{3}{*}{13} & Predictive Maintenance & & \\
\hline & 1) Ignition system fautt code generation & & \\
\hline & 2) Prognostics on finite life devices & & \\
\hline & Soundon't ond on 13 & & \\
\hline 14 & So we don't end on 13. & & \\
\hline & & & \\
\hline & & & \\
\hline
\end{tabular}

* $1.5 \mathrm{MWe}$ engine operating at $1800 \mathrm{rpm}$. 


\section{Appendix-B: Equipment Data Sheets}

\section{Vibration Exciter}

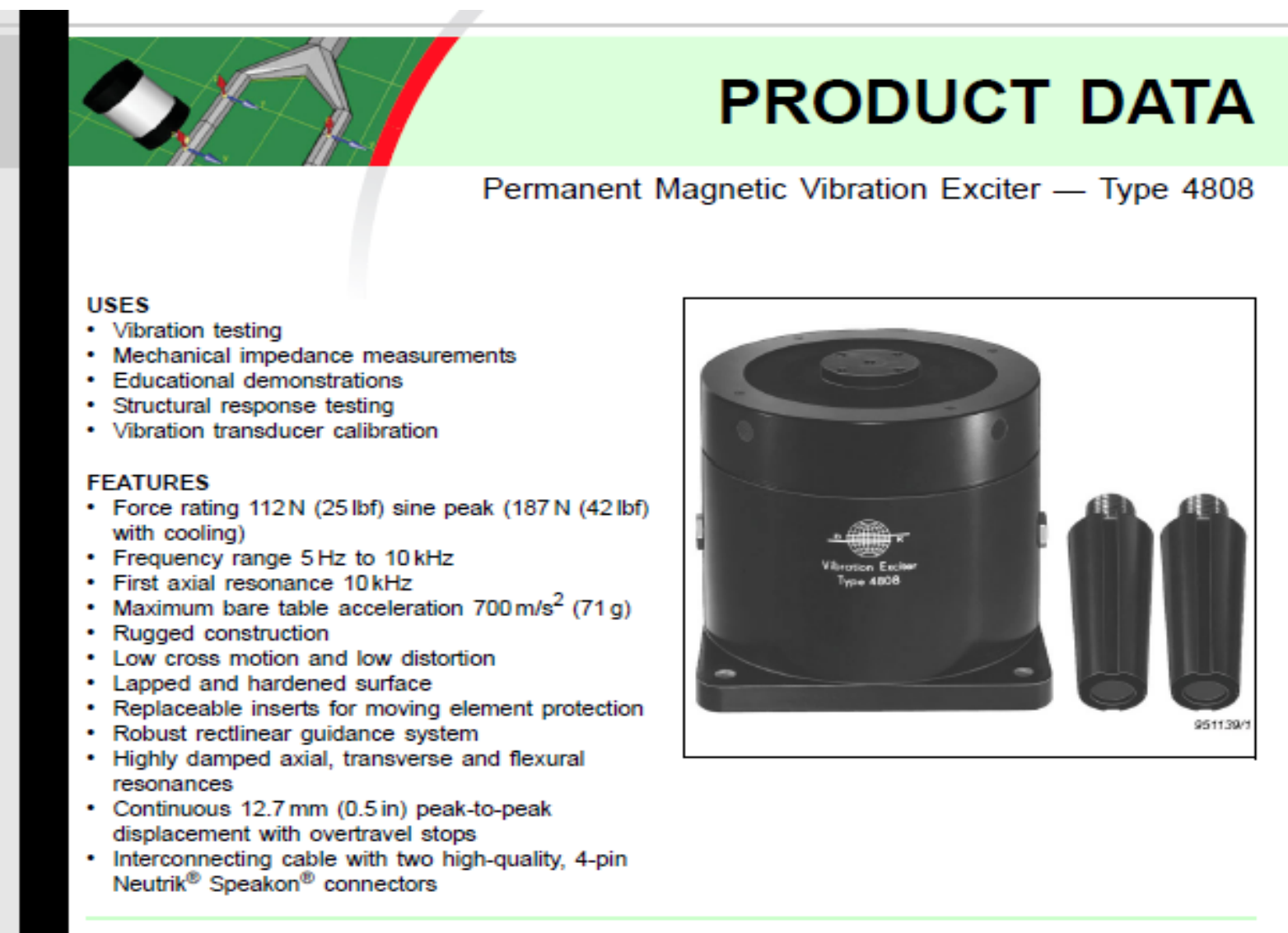

\section{Description}

Permanent Magnetic Vibration Exciter Type 4808 is a high-quality, compact machine with a permanent magnetic field. It is designed for long, trouble-free operation, and has a force rating of 112 newton ( $25 \mathrm{lbf}$ ) enabling relatively heavy loads to be excited to high g levels. Type 4808 will normally be driven by Power Amplifier Type 2719 rated at $180 \mathrm{VA}$, but can also be driven by any amplifier up to a maximum input current of 15 A RMS without assisted cooling.

The moving element is supported by a robust rectilinear guidance system consisting of grouped radial and transverse flexures in a unique construction. The flexures are made from a bonded sandwich of spring steel and a damping elastomer, providing a clean acceleration waveform with low cross motion and low distortion characteristics. 
Fig. 1

Sine performance curves for Vibration Exciter Type 4808 for operation without assisted cooling

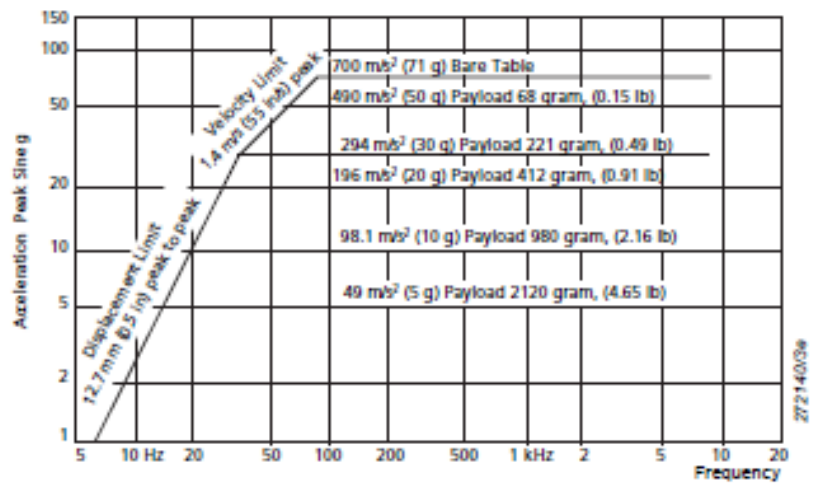

Specifications - Permanent Magnetic Vibration Exciter Type 4808

\section{COMPLIANCE WITH STANDARDS}

C f Compliance with EMC Directive Compliance with EMC Requirements of Australia and New Zealand

Safety, EMC Emission and Immunity: According to relevant standards:

EN61010-1, IEC61010-1, UL3111-1.

EN $50081-1 / 2$, IEC $61000-6-1 / 2 / 3 / 4$,

EN61326-1, CISPR 22 Class B limits, FCC

Rules Part 15, EN50082-1/2. EN61326-1

Temperature: According to IEC 60068-2-1

\& IEC 60068-2-2

Operating temperature: +5 to $+40^{\circ} \mathrm{C}(+41$ to $+104 \circ$ F)

Storage temperature: -25 to $+70=\mathrm{C}(-13$ to $+158^{\circ} \mathrm{F}$ )

Humidity: According to IEC 60088-2-3,
Damp Heat: $90 \%$ RH (non-condensing at $\left.40^{\circ} \mathrm{C}\left(104^{\circ} \mathrm{F}\right)\right)$

Mechanical: Non-operating according to

IEC 60088-2-6, IEC 60068-2-27, IEC 60088-2-29

SPECIFICATIONS

Rated Force: $112 \mathrm{~N}, 25$ lbf sine-peak (wth assisted air cooling $187 \mathrm{~N}, 42$ lbf peak)

Frequency Range: $5 \mathrm{~Hz}$ to $10 \mathrm{kHz}$ bare table Axial Resonant Frequency. $10 \mathrm{kHz}$ bare table Max Bare Table Acceleration: $700 \mathrm{~m} / \mathrm{s}^{2}(71 \mathrm{~g})$ Max. Displacement: $12.7 \mathrm{~mm}$ ( $0.5 \mathrm{in})$ peakto-peak

Max. Velocity. $1.4 \mathrm{~m} / \mathrm{s}$ ( $55 \mathrm{in} / \mathrm{s}$ )

Dynamic Weight of Moving Element:

160 gram $(0.35 \mathrm{lb})$

Static Flexure Stiffness: $5.6 \mathrm{~N} / \mathrm{mm}$ ( $32 \mathrm{~b}$ b/in)

Maximum Input Current: 15 A RMS (with assisted air cooling 25 A RMS)
Current-To-Force Ratio:

Coils in parallel: approximately $0.16 \mathrm{AVF}$ (sine peak).

Coils in series: approximately $0.08 \mathrm{~A} / \mathrm{F}$ (sine peak)

Stray Magnetic Field

$20 \times 10^{-3}$ Tesla at table face $8 \times 10^{-3}$ Tesla at $12.7 \mathrm{~mm}(0.5 \mathrm{in})$ above table face

Coil Impedance: Approximately $0.8 \Omega$ at $500 \mathrm{~Hz}$ with bare table and coils in paralel Table Size: $62.5 \mathrm{~mm}$ ( $2.45 \mathrm{in})$ diameter Fastening Thread: $5 \times 5 / 10^{\prime}-18$ UNC for M5 and 10-32 UNF inserts. 1 central insert plus 4 equi-spaced on circle of $\varnothing 50.8 \mathrm{~mm}$ WEIGHT AND DIMENSIONS

Weight: $35 \mathrm{~kg}(77.1 \mathrm{lb}$.)

Diameter: $215 \mathrm{~mm}(8.46 \mathrm{in})$ Height: $200 \mathrm{~mm}(7.87 \mathrm{in})$

\section{Ordering Information}

\begin{tabular}{|c|c|c|c|}
\hline Type 4808 & $\begin{array}{l}\text { Permanent Magnetic } \\
\text { Vibration Exciter }\end{array}$ & & $\begin{array}{l}\text { Five } \\
\text { stinge }\end{array}$ \\
\hline \multirow{4}{*}{\multicolumn{2}{|c|}{ 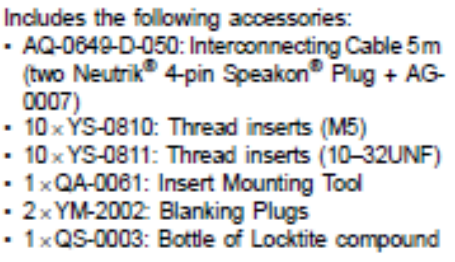 }} & $\begin{array}{l}\text { Type 8001: } \\
\text { Type 8230-C-0 }\end{array}$ & $\begin{array}{l}\text { d } \\
\text { er, Charge } \\
\text { V range) }\end{array}$ \\
\hline & & & \\
\hline & & & $\begin{array}{l}\text { Foroe } 7 \\
(+220\end{array}$ \\
\hline & & & $\begin{array}{l}\text { Force Transoucer, Detation } \\
(+2200 \text { (-2200 N range) }\end{array}$ \\
\hline \multirow{4}{*}{$\begin{array}{l}\text { OPTIONAL AC } \\
\text { WZ-0066 } \\
\text { UA-1596 }\end{array}$} & & Type 8230-001 & eltatron \\
\hline & & & \\
\hline & & & \\
\hline & & & Power Amplifier 180VA \\
\hline
\end{tabular}

UA-0125

\section{WA-0309}

UA-2054

UA-2052

CABLES AND CONNECTORS WITH OLD TYPE 2712

JJ-0500

Plug must be soldered onto AQ-0649 Cable where one Neutrik 4-pin Speakon plug is first removed

TRADEMARKS

Neutrik and Speakon are registered trademarks of Neutrik AG

Briel 8 M

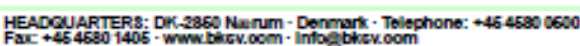

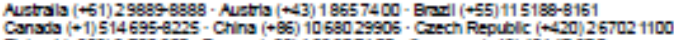

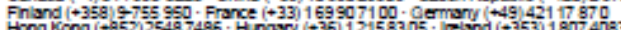

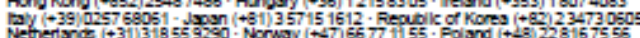

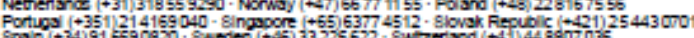

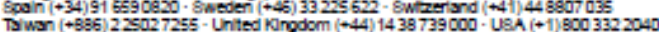




\section{Vibration Exciter Power Amplifier}

\section{Power Amplifier}

Power Amplifier Type 2719 is designed to drive small vibration exciters, particularly Vibration Exciter Type 4808. The RMS output-current limit is adjustable, making Type 2719 equally suitable to drive Vibration Exciter Type 4809 safely to full rating.

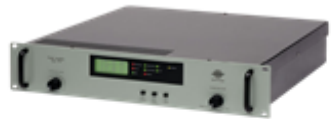

\section{由roduct Data}

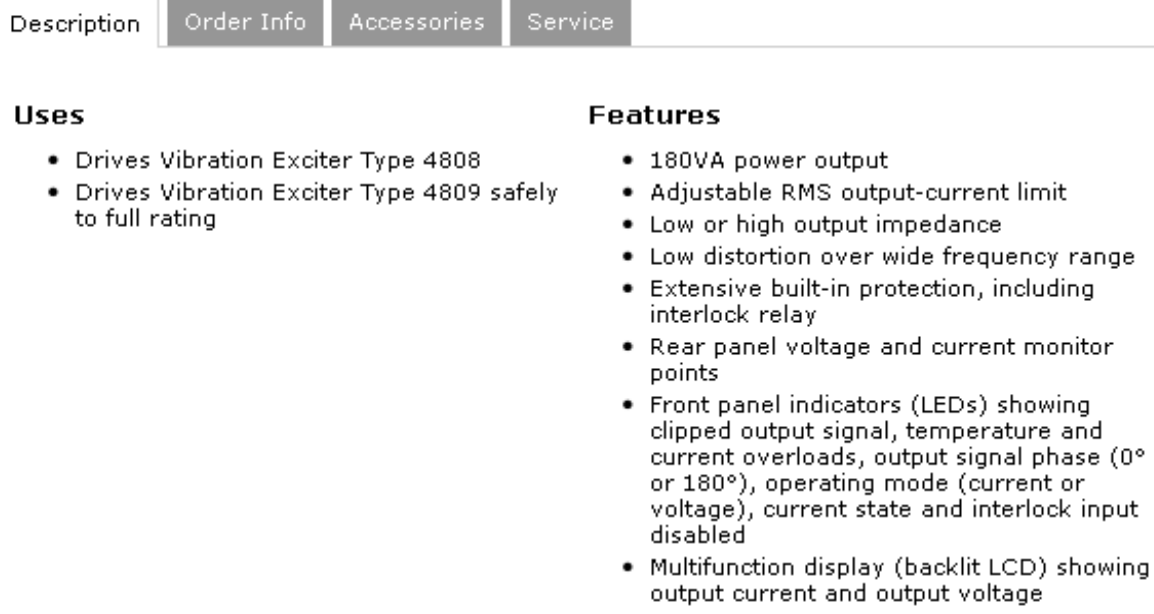

\section{Uses}

- Drives Vibration Exciter Type 4808

- Drives Vibration Exciter Type 4809 safely to full rating

\section{Features}

- 180VA power output

- Adjustable RMS output-current limit

- Low or high output impedance

- Low distortion over wide frequency range

- Extensive built-in protection, including interlock relay

- Rear panel voltage and current monitor points

- Front panel indicators (LEDs) showing clipped output signal, temperature and current overloads, output signal phase $\left(0^{\circ}\right.$ or $180^{\circ}$ ), operating mode (current or voltage), current state and interlock input disabled

- Multifunction display (backlit LCD) showing output current and output voltage

The power amplifier has a usable frequency range from $D C$ to $100 \mathrm{kHz}$. The rated $\mathrm{AC}$ output is $180 \mathrm{VA}$ into a $0.8 \Omega$ exciter or resistive load, in the frequency range $\mathrm{DC}$ to $15 \mathrm{kHz}$ ( $\pm 0.5 \mathrm{~dB}$ ). The maximum voltage gain is $14 \mathrm{~dB}$. The harmonic content of the output is very small as heavy negative feedback is used.

The instrument can tolerate temperature and supply-line variations while maintaining excellent stability. Two output modes are selectable via the front panel. The power output stage is directly coupled to the output, and hence to the connected vibration exciter. A current-limiting circuit prevents excessive instantaneous output current peaks. During operation, the voltage, current levels and waveforms can be inspected at the monitor points on the rear panel or RMS readings can be obtained from the LCD display.

Type 2719 consists of an input stage (both AC-coupled and direct), a preamplifier, a power amplifier and various warning and safety circuits with indication lamps. A multifunction display shows output current and output voltage. The amplifier can be used as a voltage generator with low output impedance and a flat voltage frequency response, or as a current generator with high output impedance and a flat current frequency response.

For a full Description and Specifications, see 9 Product Data

$\checkmark$ Contact us by phone

Ask a question

REQUEST A QUOTE

We help our customers measure and manage the quality of sound and vibration in their products and in the environment 


\section{$\underline{\text { Accelerometer }}$}

\section{$\mathrm{CH}$ Robotics}

UM6 Ultra-Miniature Orientation Sensor Datasheet

Document rev. 2.2 , updated $8 / 28 / 2012$

\section{Introduction}

Device Overview

The UM6 Ultra-Miniature Orientation Sensor combines sensor measurements from rate gyros, accelerometers, and magnetic sensors to measure orientation at $500 \mathrm{~Hz}$. The UM6 also has the capability to interface with external GPS modules to provide position, velocity, course, and speed information.

Communication with the UM6 is performed over either a TTL (3.3V) UART or a SPI bus.

The UM6 is configured by default to automatically transmit data over the UART. The UM6 can be configured to automatically transmit raw sensor data, processed sensor data, angle estimates, and angle estimate covariances at user configurable rates ranging from $20 \mathrm{~Hz}$ to $300 \mathrm{~Hz}$ in roughly $1 \mathrm{~Hz}$ increments. The UM6 can also receive and parse GPS packets, automatically transmitting new GPS position, velocity, and satellite data whenever it is available. Alternatively, the UM6 can operate in "silent mode," where data is transmitted only when specific requests are received over the UART. Regardless of the transmission mode and rate, internal angle estimates are updated at $500 \mathrm{~Hz}$ to improve accuracy.

The UM6 simplifies integration by providing a number of automatic calibration routines, including rate gyro bias calibration, magnetometer hard and soft iron calibration, and accelerometer "zeroing" to compensate for sensor-platform misalignment. All calibration routines are triggered by sending simple commands over the serial interface.

The UM6 comes factory-calibrated to remove soft and hard iron distortions present in the enclosure. When integrated into the end-user system, additional calibration may be necessary to correct other magnetic field distortions. Magnetometer calibration can be performed using the UM6 interface software, available for free download from www.chrobotics.com/downloads.

Temperature compensation of rate gyro biases is also supported by the UM6. An internal temperature sensor is used to measure temperature, and third-order compensation is applied to remove the effects of temperature-induced bias. By default, the terms used in compensation are all zero, which means that no temperature compensation is performed. The compensation terms must be determined experimentally by the end-user. On special request, compensation can be performed on each device at the factory.

The UM6 can be configured to use either Euler Angles or quaternions for attitude estimation. In Euler Angle mode, magnetometer updates are restricted to yaw alone. This can be useful in cases where distortions are possible or even expected, and where it would be undesirable for those distortions to affect pitch and roll angles (i.e. on a flying rotorcraft). In quaternion mode, Euler Angles are still available, but there are no restrictions on what angles the magnetometer is allowed to influence. 


\section{$\mathrm{CH}$ Robotics}

UM6 Ultra-Miniature Orientation Sensor Datasheet

Document rev. 2.2 , updated $8 / 28 / 2012$

Table 6 - UM6 Pin Descriptions (side connector)

\begin{tabular}{|c|c|l|}
\hline Pin $\#$ & Pin Name & Description \\
\hline 1 & Vdd & Input voltage (3.5V to 5V) \\
\hline 2 & GND & Supply ground \\
\hline 3 & RX & UART RX pin (input) \\
\hline 4 & TX & UART TX pin (output) \\
\hline
\end{tabular}

\section{Mechanical Drawings}

\section{Top View}

$\mathrm{mm}$ (inches)

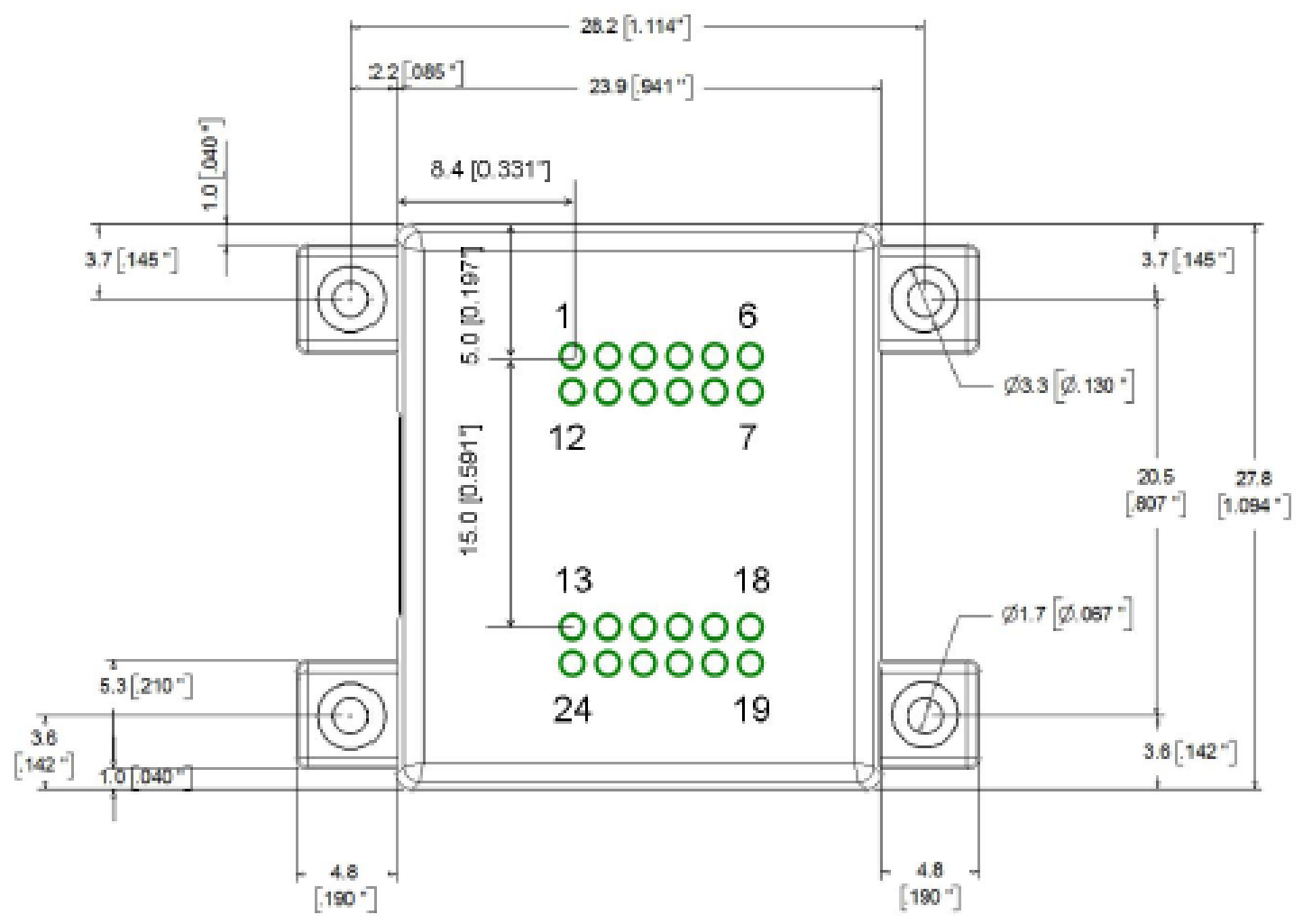

Part number 609-2890-ND from Digi-Key is a compatible $2 \mathrm{~mm}$ pin header for connecting to the bottom connectors on the UM6. 


\section{Heat Tape}

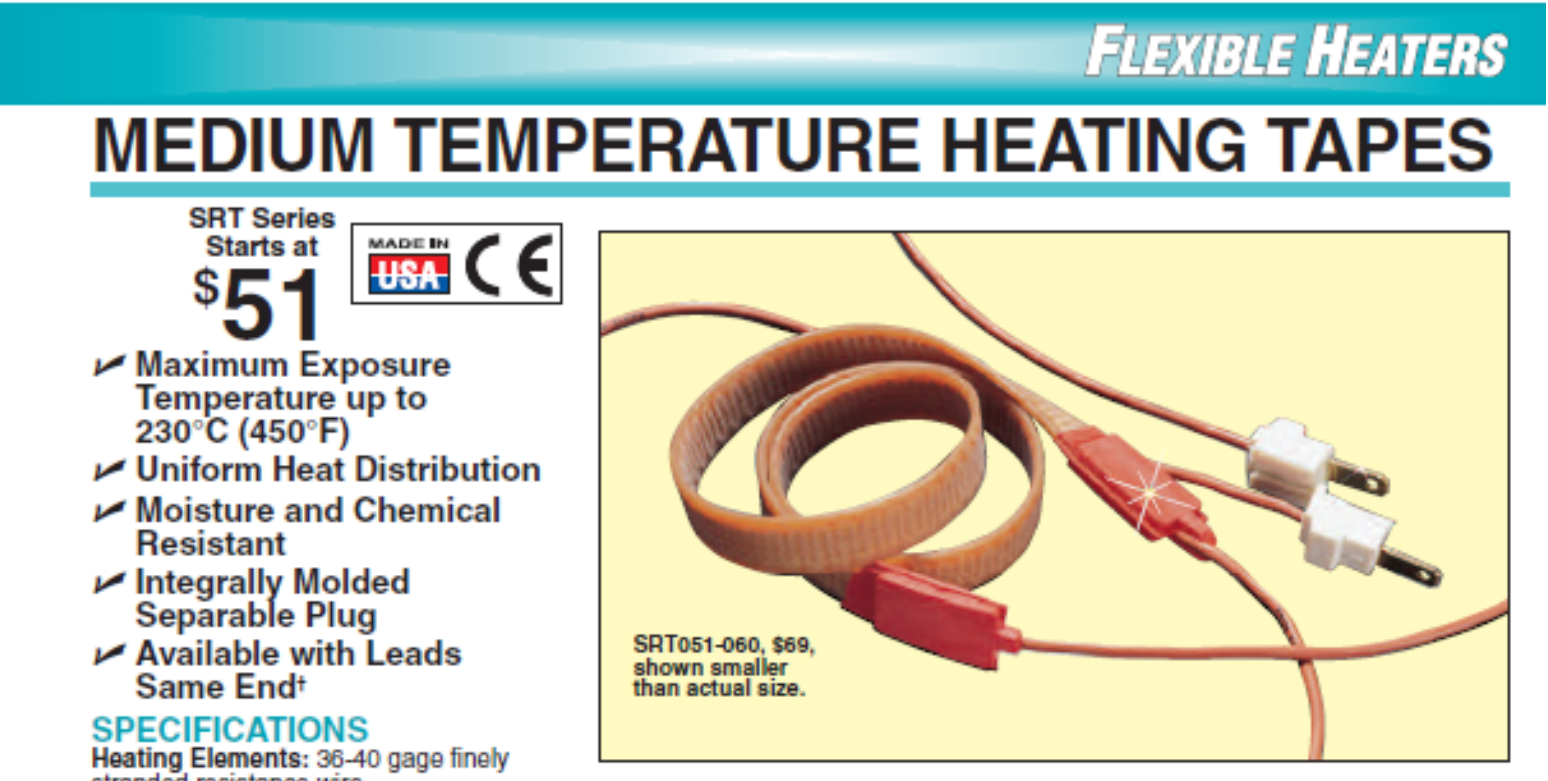

Heating Elements: 36-40 gage finely

stranded resistance wire

Heating Elements Insulation:

Double braided fiberglass yarn

Dielectric Strength: In excess of $2000 \mathrm{~V}$

Lead Wires: 16 AWG high temperature

$600 \mathrm{~V}$ silicone rubber insulated lead wire

emerging from opposite ends of the

heating tape into separate sides of

integrally molded separable plug

OMEGALUX" silicone rubber

extruded heating tapes are low-

watt density electrical resistance

heaters designed for temperature

maintenance in applications

requiring moisture and chemical

resistance. Silicone rubber tapes

are constructed of finely stranded

resistance wires fully insulated with

braided fiberglass and knitted into

flat tape with fiberglass yarn. These

tapes are encapsulated in a void-

free silicone rubber sheath.

t To onder heaters with power leads exiting the

same end of the tape, add suffix $e^{2}-L S E$ to

model number. Cal Sales for priaing To onder

240 V version change the "1" before the "In

model number to 25 . All $240 \mathrm{~V}$ versions are supplied without plugs. Call for saks prices.
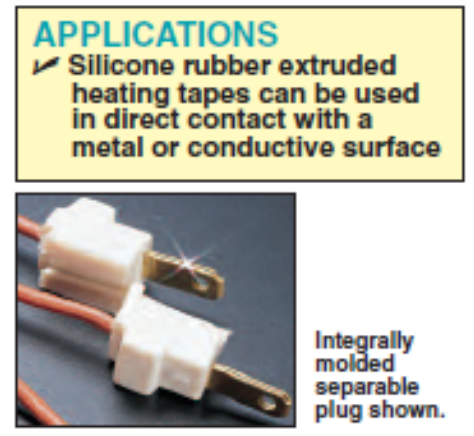

\section{Integrally}

molded

separable
plug shown.

\begin{tabular}{|c|c|c|c|c|c|}
\hline Watts & $\mathrm{W} / \mathrm{in}^{2}$ & Volts & Slze cm/m ("r) & Model No. & Price \\
\hline 52 & 4.3 & 120 & $1 / 0.6(1 / 2)$ & SRT051-020 & $\$ 51$ \\
\hline 104 & 4.3 & 120 & $1 / 1.2(5 / 4)$ & SRT051-040 & 58 \\
\hline 156 & 4.3 & 120 & $1 / 1.8(5 / 6)$ & SRT051-060 & 69 \\
\hline 209 & 4.3 & 120 & $1 / 2.4(\% / 8)$ & SRT051-080 & 80 \\
\hline 261 & 4.3 & 120 & $1 / 3(1 / / 10)$ & SRT051-100 & 92 \\
\hline 313 & 4.3 & 120 & $1 / 3.7(1 / 2 / 12)$ & SRT051-120 & 83 \\
\hline 104 & 4.3 & 120 & $2.5 / 0.6(1 / 2)$ & SRT101-020 & 69 \\
\hline 209 & 4.3 & 120 & $2.5 / 1.2(1 / 4)$ & SRT101-040 & 86 \\
\hline 313 & 4.3 & 120 & $2.5 / 1.8(1 / 6)$ & SRT101-060 & 109 \\
\hline 418 & 4.3 & 120 & $2.5 / 2.4(1 / 8)$ & SRT101-080 & 126 \\
\hline 522 & 4.3 & 120 & $2.5 / 3(1 / 10)$ & SRT101-100 & 144 \\
\hline 627 & 4.3 & 120 & $2.5 / 3.7(1 / 12)$ & SRT101-120 & 154 \\
\hline 731 & 4.3 & 120 & $2.5 / 3.7(1 / 12)$ & SRT101-140 & 177 \\
\hline 836 & 4.3 & 120 & $2.5 / 4.9(1 / 16)$ & SRT101-160 & 189 \\
\hline 940 & 4.3 & 120 & $2.5 / 5.5(1 / 18)$ & SRT101-180 & 207 \\
\hline 1045 & 4.3 & 120 & $2.5 / 6.1(1 / 20)$ & SRT101-200 & 224 \\
\hline 209 & 4.3 & 120 & $5 / 0.6(2 / 2)$ & SRT201-020 & 92 \\
\hline 418 & 4.3 & 120 & $5 / 1.2(2 / 4)$ & SRT201-040 & 132 \\
\hline 627 & 4.3 & 120 & $5 / 1.8(2 / 6)$ & SRT201-060 & 184 \\
\hline 836 & 4.3 & 120 & $5 / 2.4(2 / 8)$ & SRT201-080 & 230 \\
\hline 1045 & 4.3 & 120 & $5 / 3(2 / 10)$ & SRT201-100 & 252 \\
\hline 1254 & 4.3 & 120 & $5 / 3.7(2 / 12)$ & SRT201-120* & 275 \\
\hline 1463 & 4.3 & 120 & $5 / 4.3(2 / 14)$ & SRT201-140" & 293 \\
\hline 1672 & 4.3 & 120 & $5 / 4.9(2 / 16)$ & SRT201-160" & 333 \\
\hline 1881 & 4.3 & 120 & $5 / 5.5(2 / 18)$ & SRT201-180" & 379 \\
\hline 2090 & 4.3 & 120 & $5 / 6.1(2 / 20)$ & SRT201-200* & 407 \\
\hline
\end{tabular}

* Does not come with piug.

Comes complete with instruction sheet.

Ordering Examples: SRT051-060, 120V, 156 Watt, heating tape, $\$ 60$.

SRT051-04Q, 120V, 104 Wheating tape, $\$ 58$. 


\section{J-type Thermocouples}

\section{"Cement-On" Thermocouples}

$\checkmark$ Response Time in Milliseconds

$\sim$ Made from $0.013 \mathrm{~mm}\left(0.0005^{\prime \prime}\right)$ Foil and $0.25 \mathrm{~mm}\left(0.010^{\prime \prime}\right)$ Diameter Thermocouple Wire

$\sim$ Very Low Thermal Inertia

$\sim$ Four Calibrations J, K, E, and T

$\checkmark$ Three Styles Ideal for Surface Measurement
OMEGA introduces its Cement-On, fast response thermocouples for fast surface temperature measurement applications in three convenient styles. Styles 1 and 2 are made from $0.013 \mathrm{~mm}\left(0.0005^{\prime \prime}\right)$ thermocouple alloy foil by a special process where the butt welded thermocouple junction is $0.013 \mathrm{~mm}\left(0.0005^{5}\right)$ in thickness. Styles 1 and 2 are flat, extremely low inertia construction and are an ideal means of measuring the temperature of both flat and curved metals, plastic and ceramic surfaces where very fast response is desired.

OMEGA's Cement-On Style 1 and 2 thermocouples are fabricated from ANSI "Special Limits of Error" grade thermocouple materials in K, E and T calibrations and yield accurate temperature indication when used with standard thermocouple instrumentation. Styles 1 and 2 have the fastest response. Style 3 is an economy version constructed from $0.25 \mathrm{~mm}\left(0.010^{\circ}\right)$ diameter bead welded standard limit of error thermocouple wire. It should be used where extremely fast response is not essential. Hont $1 \mathrm{~m}\left(40^{n}\right)$ standard

Style 2

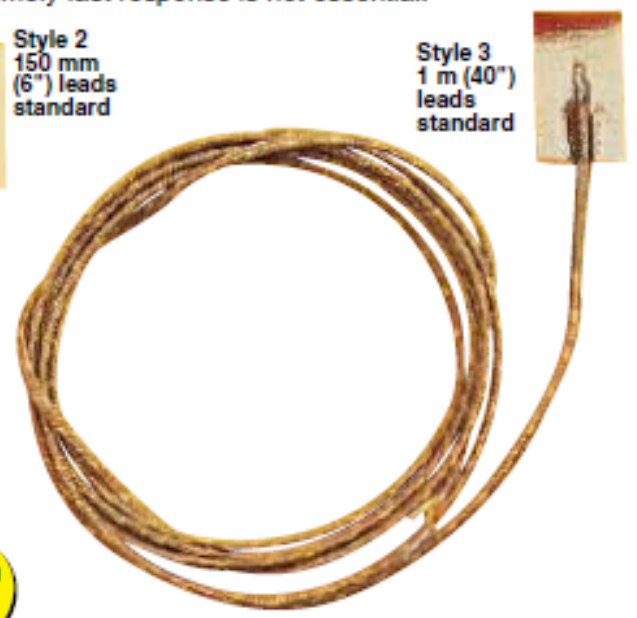

\begin{tabular}{|c|c|c|c|c|c|c|}
\hline Model No. & Style & Thermocouple Type & Length & Continuous & $600 \mathrm{hr}$ & $10 \mathrm{hr}$ \\
\hline CO1-K & & $K \quad$ CHROMEGA* $^{*}$-ALOMEGA* & $1 \mathrm{~m}\left(40^{\prime \prime}\right)$ & $260(500)$ & $315(600)$ & $370(700)$ \\
\hline CO1-E & & E CHROMEGA*-Constantan & $1 \mathrm{~m}\left(40^{\prime \prime}\right)$ & $260(500)$ & $315(600)$ & $370(700)$ \\
\hline CO1-T & & T Copper - Constantan & $1 \mathrm{~m}\left(40^{\prime \prime}\right)$ & $150(300)$ & $205(400)$ & $260(500)$ \\
\hline $\mathrm{CO} 2-\mathrm{E}$ & & 들 CHROMEGA*-Constantan & $150 \mathrm{~mm}\left(6^{\prime \prime}\right)$ & $425(800)$ & $425(800)$ & $540(1000)$ \\
\hline $\mathrm{CO} 2-\mathrm{T}$ & & T Copper-Constantan & $150 \mathrm{~mm}\left(6^{\prime \prime}\right)$ & $150(300)$ & $150(300)$ & $260(500)$ \\
\hline $\mathrm{CO}-\mathrm{J}$ & & J Iron - Constantan & $1 \mathrm{~m}\left(40^{\prime \prime}\right)$ & $260(500)$ & $370(700)$ & $370(700)$ \\
\hline $\mathrm{CO} 3-\mathrm{K}$ & & K CHROMEGA*-ALOMEGA* $^{*}$ & $1 \mathrm{~m}\left(40^{\prime \prime}\right)$ & $260(500)$ & $370(700)$ & $370(700)$ \\
\hline $\mathrm{CO} 3-\mathrm{E}$ & & 巨 CHROMEGA*-Constantan & $1 \mathrm{~m}\left(40^{\prime \prime}\right)$ & $260(500)$ & $370(700)$ & $370(700)$ \\
\hline
\end{tabular}

* The temperature range high limits given are greatly influenced by environmental conditions, installation method, accuracy and lifetime requirements and may vary from the general guidelines listed in the table.

Style 1 and 3 cannot be used with CC High Temperature Cement; CC Cement will break down insulation.

Response time when "gounded" $\alpha$ "cemented" to surface: Style 1 (10 to 20 milliseconds), Style 2 (2 to 5 milliseconds), Style 3 (300 miliseconds). The response time or "time constant" is the time required to reach $63.2 \%$ of an instantaneous temperature change.

Additional length wie can be ordered for Styles 1 and 3, add cost per $300 \mathrm{~mm}$ (12"), for Style 2 add cost per $300 \mathrm{~mm}$ (12\%).

Ordering Example: CO1-K is a style 1, Type $K$ thermocouple, $1 \mathrm{~m}\left(40^{\circ}\right)$ long. 
i.Series @E MONOGRAM"

$\checkmark$ High Qualty

V 5-Year Warranty

$\checkmark$ High Accuracy: $\pm 0.5^{\circ} \mathrm{C}$ $\left( \pm 0.9^{\circ} \mathrm{F}\right), 0.03 \%$ Reading

$\checkmark$ User-Friendly, Simple to Configure

$\checkmark$ Free Software

$\checkmark$ Full Autotune PID Control

$\checkmark$ Universal Inputs: RTD, Thermocouple, Process Voltage/Current, Strain

$\checkmark$ Totally Programmable Color Displays Standard

$\checkmark$ Built-In Excitation Standard

$\checkmark 2$ Control or Alarm Outputs: DC Pulse, Mechanical Relays, Analog Voltage and Current

$\checkmark$ Embedded Internet Connectivity Available

The OMEGA* CNis is a $1 / 6$ DIN size [ $\left.96 \times 48 \mathrm{~mm}\left(3.7 \times 1.9^{\prime \prime}\right)\right]$ digital panel meter featuring the big ISeries color-changing display. The digits are twice the size of typical '7 DIN panel meters. The iSeries meters feature the only LED displays that can be programmed to change color between GREEN. $A M B E F$, and RED at any setpoint or alarm point. The "CNi8" model is available as an extremely accurate programmable digital panel meter with no outputs or with dual outputs for controlling or alarming functions. Other options include isolated programmable analog output, serial communications, MODBUS and Ethernet. The user can easily program the $\mathrm{CNi}$ for any control or alarming requirement from simple orvoff to full autotune PID with a choice of form C SPDT relays, solid state relays, DC pulse, and analog (voltage and current) outputs.

Fully isolated analog output for retransmission of the process value is available in addition to the control and alarm relays (specify model CNi8A33).

The CNi8 covers a broad selection of transducer and transmitter inputs with 2 input models.

\section{$1 / 8$ DIN Temperature, Process and Strain PID Controllers}

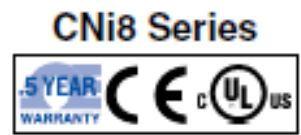

The universal temperature and process instrument (CNi models) handles 10 common types of thermocouples, multiple RTDs, and several process (DC) voltage and current ranges. This model also features built-in excitation, $24 \mathrm{Vdc}$ (2) $25 \mathrm{~mA}$. With its wide choice of signal inputs, this model is an excellent choice for measuring or controlling temperature with a thermocouple, RTD, or 4 to $20 \mathrm{~mA}$ transmitter.

The strain and process instruments (CNiS models) measure inputs from load cells, pressure transducers, and most any strain gage sensor as well as process voltage and

\begin{tabular}{|c|c|c|c|}
\hline & Input Type & Range & Accuracy \\
\hline \multicolumn{4}{|c|}{ Universal Process } \\
\hline & Process Voltage & 0 to $100 \mathrm{mV}, 0$ to $1 \mathrm{~V}, 0$ to $10 \mathrm{Vdc}$ & $0.03 \%$ rdg \\
\hline & Process Current & 0 to $20 \mathrm{~mA}$ ( 4 to $20 \mathrm{~mA}$ ) & $0.03 \%$ rdg \\
\hline & Excitation & $24 \mathrm{~V} \mathrm{e} 25 \mathrm{~mA}$ & - \\
\hline \multicolumn{4}{|c|}{ Universal Strain/Process } \\
\hline & Process Voltage & 0 to $100 \mathrm{mV},-100$ to $1 \mathrm{~V}, 0$ to $10 \mathrm{Vdc}$ & $0.03 \%$ rdg \\
\hline & Process Current & 0 to $20 \mathrm{~mA}$ ( 4 to $20 \mathrm{~mA}$ ) & $0.03 \%$ rdg \\
\hline & Excitation & $5 \mathrm{~V} 640 \mathrm{~mA}, 10 \mathrm{~V} \approx 60 \mathrm{mV}$ & - \\
\hline \multicolumn{4}{|c|}{ Nickel RTD Input (FS Required) } \\
\hline RTD & -1N (Nickel MIL-T-7990B) & 0 to $200^{\circ} \mathrm{C}\left(32\right.$ to $\left.392^{\circ} \mathrm{F}\right)$ & $0.1^{\circ} \mathrm{C}\left(0.2^{\circ} \mathrm{F}\right)$ \\
\hline RTD & -2N (Nickel MIL-T-7990B) & -40 to $300^{\circ} \mathrm{C}\left(-40\right.$ to $\left.572^{\circ} \mathrm{F}\right)$ & $0.3^{\circ} \mathrm{C}\left(0.5^{\circ} \mathrm{F}\right)$ \\
\hline \multicolumn{4}{|c|}{ Temperature } \\
\hline $\mathrm{J}$ & Iron-Constantan & -210 to $760^{\circ} \mathrm{C}\left(-346\right.$ to $\left.1400^{\circ} \mathrm{F}\right)$ & $0.4^{\circ} \mathrm{C}\left(0.7^{\circ} \mathrm{F}\right)$ \\
\hline K & CHROMEGA-ALOMEGA- & $\begin{array}{l}-270 \text { to }-160^{\circ} \mathrm{C} /-160 \text { to } 1372^{\circ} \mathrm{C} \\
\left(-454 \text { to }-256^{\circ} \mathrm{F} /-256 \text { to } 2502^{\circ} \mathrm{F}\right)\end{array}$ & $\begin{aligned} & 1.0^{\circ} \mathrm{C} / 0.4^{\circ} \mathrm{C} \\
&\left(1.8^{\circ} \mathrm{F} / 0.7^{\circ} \mathrm{F}\right.\end{aligned}$ \\
\hline Т & Copper-Constantan & $\begin{array}{l}-270 \text { to }-190^{\circ} \mathrm{C}-190 \text { to } 400^{\circ} \mathrm{C} \\
\left(-454 \text { to }-310^{\circ} \mathrm{F} /-310 \text { to } 752^{\circ} \mathrm{F}\right)\end{array}$ & $\begin{array}{l}1.0^{\circ} \mathrm{C} / 0.4^{\circ} \mathrm{C} \\
\left(1.8^{\circ} \mathrm{F} / 0.7^{\circ} \mathrm{F}\right)\end{array}$ \\
\hline $\mathbf{E}$ & CHROMEGA"-Constantan & $\begin{array}{l}-270 \text { to }-220^{\circ} \mathrm{C} /-220 \text { to } 1000^{\circ} \mathrm{C} \\
\left(-454 \text { to }-364^{\circ} \mathrm{F} /-364 \text { to } 1832^{\circ} \mathrm{F}\right)\end{array}$ & $\begin{aligned} & 1.0^{\circ} \mathrm{C} / 0.4^{\circ} \mathrm{C} \\
&\left(1.8^{\circ} \mathrm{F} / 0.7^{\circ} \mathrm{F}\right.\end{aligned}$ \\
\hline $\mathbf{R}$ & $\mathrm{Pt} / 13 \% \mathrm{Rh}-\mathrm{Pt}$ & $\begin{array}{c}-50 \text { to } 40^{\circ} \mathrm{C} / 40 \text { to } 1768^{\circ} \mathrm{C} \\
\left(-58 \text { to } 104^{\circ} \mathrm{F} / 104 \text { to } 3214^{\circ} \mathrm{F}\right)\end{array}$ & $\begin{aligned} 1.0^{\circ} \mathrm{C} / 0.5^{\circ} \mathrm{C} \\
\left(1.8^{\circ} \mathrm{F} / 0.9^{\circ} \mathrm{F}\right. \\
\end{aligned}$ \\
\hline $\mathbf{S}$ & $\mathrm{Pt} / 10 \% \mathrm{Rh}-\mathrm{Pt}$ & $\begin{array}{l}-50 \text { to } 100^{\circ} \mathrm{C} / 100 \text { to } 1768^{\circ} \mathrm{C} \\
\left(-58 \text { to } 212^{\circ} \mathrm{F} / 212 \text { to } 3214^{\circ} \mathrm{F}\right)\end{array}$ & $\begin{array}{l}1.0^{\circ} \mathrm{C} / 0.5^{\circ} \mathrm{C} \\
\left(1.8^{\circ} \mathrm{F} / 0.9^{\circ} \mathrm{F}\right.\end{array}$ \\
\hline B & $30 \%$ Rh-Pt/6\%Rh-Pt & $\begin{array}{l}100 \text { to } 640^{\circ} \mathrm{C} / 640 \text { to } 1820^{\circ} \mathrm{C} \\
\left(212 \text { to } 1184^{\circ} \mathrm{F} / 1184 \text { to } 3308^{\circ} \mathrm{F}\right)\end{array}$ & $\begin{array}{l}1.0^{\circ} \mathrm{C} / 0.5^{\circ} \mathrm{C} \\
\left(1.8^{\circ} \mathrm{F} / 0.9^{\circ} \mathrm{F}\right.\end{array}$ \\
\hline C & $5 \% R e-W / 26 \% R e-W$ & 0 to $2320^{\circ} \mathrm{C}$ (32 to $4208^{\circ} \mathrm{F}$ ) & $0.4^{\circ} \mathrm{C}\left(0.7^{\circ} \mathrm{F}\right)$ \\
\hline $\mathbf{N}$ & Nicrosil-nisil & $\begin{array}{l}-250 \text { to }-100^{\circ} \mathrm{C} /-100 \text { to } 1300^{\circ} \mathrm{C} \\
\left(-418 \text { to }-148^{\circ} \mathrm{F} /-148 \text { to } 2372^{\circ} \mathrm{F}\right)\end{array}$ & $\begin{array}{l}1.0^{\circ} \mathrm{C} / 0.4^{\circ} \mathrm{C} \\
\left(1.8^{\circ} \mathrm{F} / 0.7^{\circ} \mathrm{F}\right)\end{array}$ \\
\hline & J DIN & -200 to $900^{\circ} \mathrm{C}\left(-328\right.$ to $\left.1652^{\circ} \mathrm{F}\right)$ & $0.4^{\circ} \mathrm{C}\left(0.7^{\circ} \mathrm{F}\right.$ \\
\hline 410 & 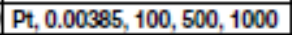 & -200 to $900^{\circ} \mathrm{C}\left(-328\right.$ to $\left.1652^{\circ} \mathrm{F}\right)$ & $0.4^{\circ} \mathrm{C}\left(0.7^{\circ} \mathrm{F}\right)$ \\
\hline RUD & 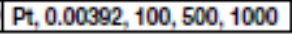 & -200 to $850^{\circ} \mathrm{C}\left(-328\right.$ to $\left.1652^{\circ} \mathrm{F}\right)$ & $0.4^{\circ} \mathrm{C}\left(0.7^{\circ} \mathrm{F}\right.$ \\
\hline
\end{tabular}

\section{P-13}

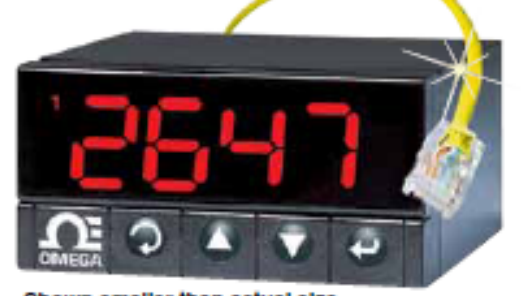

Shown smaller than actual size.

current ranges. The $\mathrm{CN}$ iS has builtin 5 or $10 \mathrm{Vdc}$ excitation for bridge transducers, 5 Vdc @ $40 \mathrm{~mA}$ or $10 \mathrm{Vdc}$ (6) $60 \mathrm{~mA}$ (any excitation voltage between 5 and $24 \mathrm{Vdc}$ is available by special order). This CNiS model supports 4- and 6-wire bridge configurations, ratiometric and non-ratiometric measurements.

The CNis features fast and easy "in process" calibration/scaling of the signal inputs to any engineering units. This model also features 10-point linearization which allows the user to linearize the signal input from extremely nonlinear transducers of all kinds. 


\section{NI 6023E/6024E/6025E Family Specifications}

This document lists the $\mathrm{L} / \mathrm{O}$ terminal summary and specifications for the devices that make up the NI 6023E/6024E/6025E family of devices. This family includes the following devices:

- $\quad$ NI PCI-6023E

- NI DAQCard-6024E

- $\quad$ NI PCI-6024E

- $\quad$ NI PCI-6025E

- NI PXI-6025E

\section{1/0 Terminal Summary}

Note With NI-DAQmx, National Instruments revised its terminal names so they are easier to understand and more consistent among NI hardware and software products. The revised terminal names used in this document are usually similar to the names they replace. For a complete list of Traditional NI-DAQ (Legacy) terminal names and their NI-DAQmx equivalents, refer to Terminal Name Equivalents of the E Series Help.

Table 1. 1/0 Terminals

\begin{tabular}{|c|c|c|c|c|c|c|c|}
\hline Terminal Name & $\begin{array}{l}\text { Terminal } \\
\text { Type and } \\
\text { Direction }\end{array}$ & $\begin{array}{c}\text { Impedance } \\
\text { Input/ } \\
\text { Output }\end{array}$ & $\begin{array}{l}\text { Protection } \\
\text { (V) On/Off }\end{array}$ & $\begin{array}{c}\text { Source } \\
(\mathrm{mA} \text { at } \mathrm{V})\end{array}$ & $\begin{array}{c}\text { Sink } \\
(\mathbf{m A} \text { at } \mathbf{V})\end{array}$ & $\begin{array}{l}\text { Rise } \\
\text { Time } \\
\text { (ns) }\end{array}$ & Bias \\
\hline $\mathrm{AI}<0 . .15>$ & $\mathrm{AI}$ & $\begin{array}{l}100 \mathrm{G} \Omega \text { in } \\
\text { parallel } \\
\text { with } 100 \mathrm{pF}\end{array}$ & $42 / 35$ & - & - & - & $\pm 200 \mathrm{pA}$ \\
\hline AI SENSE & $\mathrm{AI}$ & $\begin{array}{l}100 \mathrm{G} \Omega \text { in } \\
\text { parallel } \\
\text { with } 100 \mathrm{pF}\end{array}$ & $40 / 25$ & - & - & - & $\pm 200 \mathrm{pA}$ \\
\hline AI GND & - & - & - & - & - & - & - \\
\hline $\mathrm{AO}^{\dagger}$ & $\mathrm{AO}$ & $0.1 \Omega$ & $\begin{array}{l}\text { Short-circuit } \\
\text { to ground }\end{array}$ & 5 at 10 & 5 at -10 & $10 \mathrm{~V} / \mu \mathrm{s}$ & - \\
\hline $\mathrm{AO} 1^{\dagger}$ & $\mathrm{AO}$ & $0.1 \Omega$ & $\begin{array}{l}\text { Short-circuit } \\
\text { to ground }\end{array}$ & 5 at 10 & 5 at -10 & $10 \mathrm{~V} / \mu \mathrm{s}$ & - \\
\hline $\mathrm{AO} G N D$ & - & - & - & - & - & - & - \\
\hline D GND & - & - & - & - & - & - & - \\
\hline
\end{tabular}


Table 1. $1 / 0$ Terminals (Continued)

\begin{tabular}{|c|c|c|c|c|c|c|c|}
\hline Terminal Name & $\begin{array}{l}\text { Terminal } \\
\text { Type and } \\
\text { Direction }\end{array}$ & $\begin{array}{c}\text { Impedance } \\
\text { Input/ } \\
\text { Output }\end{array}$ & $\begin{array}{l}\text { Protection } \\
\text { (V) On/Off }\end{array}$ & $\begin{array}{c}\text { Source } \\
(\mathrm{mA} \text { at } \mathrm{V})\end{array}$ & $\begin{array}{c}\text { Sink } \\
(\mathrm{mA} \text { at } \mathrm{V})\end{array}$ & $\begin{array}{c}\text { Rise } \\
\text { Time } \\
\text { (ns) }\end{array}$ & Bias \\
\hline$+5 \mathrm{~V}$ & - & $0.1 \Omega$ & $\begin{array}{l}\text { Short-circuit } \\
\text { to ground }\end{array}$ & $1 \mathrm{~A}$ fused & - & - & - \\
\hline $\mathrm{P} 0 .<0 . .7>$ & DIO & - & $\mathrm{v}_{\mathrm{cc}}+0.5$ & $\begin{array}{l}13 \text { at } \\
\left(\mathrm{V}_{C \mathrm{CC}}-0.4\right)\end{array}$ & 24 at 0.4 & 1.1 & $50 \mathrm{k} \Omega \mathrm{pu}$ \\
\hline $\mathrm{P} 1 .<0.7>t$ & DIO & - & $\mathrm{v}_{\mathrm{cc}}+0.5$ & $\begin{array}{l}2.5 \text { at } \\
3.0 \mathrm{~min}\end{array}$ & 2.5 at 0.4 & 5 & $100 \mathrm{k} \Omega \mathrm{pu}$ \\
\hline $\mathrm{P} 2 .<0 . .7>t$ & DIO & - & $\mathrm{v}_{\mathrm{cc}}+0.5$ & $\begin{array}{l}2.5 \text { at } \\
3.0 \mathrm{~min}\end{array}$ & 2.5 at 0.4 & 5 & $100 \mathrm{k} \Omega \mathrm{pu}$ \\
\hline $\mathrm{P} 3 .<0.7>t$ & DIO & - & $\mathrm{v}_{\mathrm{cc}}+0.5$ & $\begin{array}{l}2.5 \text { at } \\
3.0 \mathrm{~min}\end{array}$ & 2.5 at 0.4 & 5 & $100 \mathrm{k} \Omega \mathrm{pu}$ \\
\hline $\begin{array}{l}\text { AI HOLD COMP or } \\
\text { AI HOLD }\end{array}$ & DO & - & - & $\begin{array}{l}3.5 \text { at } \\
\left(\mathrm{V}_{C \mathrm{CC}}-0.4\right)\end{array}$ & 5 at 0.4 & 1.5 & $50 \mathrm{k} \Omega \mathrm{pu}$ \\
\hline EXT STROBE* & DO & - & - & $\begin{array}{l}3.5 \text { at } \\
\left(\mathrm{V}_{C \mathrm{CC}}-0.4\right)\end{array}$ & 5 at 0.4 & 1.5 & $50 \mathrm{k} \Omega \mathrm{pu}$ \\
\hline $\begin{array}{l}\text { PFI } 0 / \\
\text { (AI START TRIG) }\end{array}$ & DIO & - & $\mathrm{v}_{\mathrm{cc}}+0.5$ & $\begin{array}{l}3.5 \text { at } \\
\left(V_{C C}-0.4\right)\end{array}$ & 5 at 0.4 & 1.5 & $50 \mathrm{k} \Omega \mathrm{pu}$ \\
\hline $\begin{array}{l}\text { PFI } 1 / \\
\text { (AI REF TRIG) }\end{array}$ & DIO & - & $\mathrm{v}_{\mathrm{cc}}+0.5$ & $\begin{array}{l}3.5 \text { at } \\
\left(\mathrm{V}_{\triangle C}-0.4\right)\end{array}$ & 5 at 0.4 & 1.5 & $50 \mathrm{k} \Omega \mathrm{pu}$ \\
\hline $\begin{array}{l}\text { PFI } 2 / \\
(\text { AI CONV CLK)* }\end{array}$ & DIO & - & $\mathrm{v}_{\mathrm{cc}}+0.5$ & $\begin{array}{l}3.5 \text { at } \\
\left(V_{C C}-0.4\right)\end{array}$ & 5 at 0.4 & 1.5 & $50 \mathrm{k} \Omega \mathrm{pu}$ \\
\hline $\begin{array}{l}\text { PFI 3/ } \\
\text { CTR } 1 \text { SOURCE }\end{array}$ & DIO & - & $\mathrm{v}_{\mathrm{cc}}+0.5$ & $\begin{array}{l}3.5 \text { at } \\
\left(\mathrm{V}_{\triangle C}-0.4\right)\end{array}$ & 5 at 0.4 & 1.5 & $50 \mathrm{k} \Omega \mathrm{pu}$ \\
\hline PFI 4/CTR 1 GATE & DIO & - & $\mathrm{v}_{\mathrm{cc}}+0.5$ & $\begin{array}{l}3.5 \text { at } \\
\left(\mathrm{V}_{\triangle C}-0.4\right)\end{array}$ & 5 at 0.4 & 1.5 & $50 \mathrm{k} \Omega \mathrm{pu}$ \\
\hline CTR 1 OUT & DO & - & - & $\begin{array}{l}3.5 \text { at } \\
\left(\mathrm{V}_{C \mathrm{CC}}-0.4\right)\end{array}$ & 5 at 0.4 & 1.5 & $50 \mathrm{k} \Omega \mathrm{pu}$ \\
\hline $\begin{array}{l}\text { PFI } 5 / \\
(\text { AO SAMP CLK)* }\end{array}$ & DIO & - & $\mathrm{v}_{\mathrm{cc}}+0.5$ & $\begin{array}{l}3.5 \text { at } \\
\left(\mathrm{V}_{\mathrm{CC}}-0.4\right)\end{array}$ & 5 at 0.4 & 1.5 & $50 \mathrm{k} \Omega \mathrm{pu}$ \\
\hline $\begin{array}{l}\text { PFI 6/ } \\
\text { (AO START TRIG) }\end{array}$ & DIO & - & $\mathrm{v}_{\mathrm{cc}}+0.5$ & $\begin{array}{l}3.5 \text { at } \\
\left(\mathrm{V}_{\triangle C}-0.4\right)\end{array}$ & 5 at 0.4 & 1.5 & $50 \mathrm{k} \Omega \mathrm{pu}$ \\
\hline $\begin{array}{l}\text { PFI } 7 / \\
\text { (AI SAMP CLK) }\end{array}$ & DIO & - & $\mathrm{v}_{\mathrm{cc}}+0.5$ & $\begin{array}{l}3.5 \text { at } \\
\left(V_{C C}-0.4\right)\end{array}$ & 5 at 0.4 & 1.5 & $50 \mathrm{k} \Omega \mathrm{pu}$ \\
\hline $\begin{array}{l}\text { PFI 8/ } \\
\text { CTR } 0 \text { SOURCE }\end{array}$ & DIO & - & $\mathrm{v}_{\mathrm{cc}}+0.5$ & $\begin{array}{l}3.5 \text { at } \\
\left(\mathrm{V}_{C \mathrm{CC}}-0.4\right)\end{array}$ & 5 at 0.4 & 1.5 & $50 \mathrm{k} \Omega \mathrm{pu}$ \\
\hline PFI 9/CTR 0 GATE & DIO & - & $\mathrm{v}_{\mathrm{cc}}+0.5$ & $\begin{array}{l}3.5 \text { at } \\
\left(\mathrm{V}_{C \mathrm{CC}}-0.4\right)\end{array}$ & 5 at 0.4 & 1.5 & $50 \mathrm{k} \Omega \mathrm{pu}$ \\
\hline
\end{tabular}


Table 1. I/O Terminals (Continued)

\begin{tabular}{|l|l|l|l|l|l|l|l|}
\hline Terminal Name & $\begin{array}{c}\text { Terminal } \\
\text { Type and } \\
\text { Direction }\end{array}$ & $\begin{array}{c}\text { Impedance } \\
\text { Input } \\
\text { Output }\end{array}$ & $\begin{array}{c}\text { Protection } \\
\text { (V) On/Off }\end{array}$ & $\begin{array}{c}\text { Source } \\
(\mathbf{m A} \text { at V) }\end{array}$ & $\begin{array}{c}\text { Sink } \\
\text { (mA at V) }\end{array}$ & $\begin{array}{c}\text { Rise } \\
\text { Time } \\
\text { (ns) }\end{array}$ & Bias \\
\hline \hline CTR 0 OUT & DO & - & - & $\begin{array}{l}3.5 \text { at } \\
\left(\mathrm{V}_{C C}-0.4\right)\end{array}$ & 5 at 0.4 & 1.5 & $50 \mathrm{k} \Omega \mathrm{pu}$ \\
\hline FREQ OUT & DO & - & - & $\begin{array}{l}3.5 \text { at } \\
\left(\mathrm{V}_{C C}-0.4\right)\end{array}$ & 5 at 0.4 & 1.5 & $50 \mathrm{k} \Omega \mathrm{pu}$ \\
\hline
\end{tabular}

* Indicates active low.

+ NI $6024 / 6025 \mathrm{E}$ only.

${ }^{*}$ NI $6025 \mathrm{E}$ only.

$\mathrm{AI}=$ Analog Input $\quad \mathrm{DIO}=$ Digital Input $/$ Output $\quad \mathrm{pu}=$ pull-up

$\mathrm{AO}=$ Analog Output $\quad \mathrm{DO}=$ Digital Output

Note: The tolerance on the $50 \mathrm{k} \Omega$ pull-up resistors is large. Actual value might range between $17 \mathrm{k} \Omega$ and $100 \mathrm{k} \Omega$.

\section{Specifications}

The following specifications are typical at $25^{\circ} \mathrm{C}$ unless otherwise noted.

\section{Analog Input}

\section{Input Characteristics}

Number of channels. 16 single-ended or 8 differential (software-selectable per channel)

Type of A/D converter (ADC) ......... Successive approximation

Resolution 12 bits, 1 in 4,096

Max sampling rate $.200 \mathrm{kS} / \mathrm{s}$ guaranteed

Input signal ranges (bipolar only)

\begin{tabular}{|l|l|}
\hline \multicolumn{1}{|c|}{ Range } & \multicolumn{1}{c|}{ Bipolar } \\
\hline \hline $20 \mathrm{~V}$ & $\pm 10 \mathrm{~V}$ \\
\hline $10 \mathrm{~V}$ & $\pm 5 \mathrm{~V}$ \\
\hline $1 \mathrm{~V}$ & $\pm 500 \mathrm{mV}$ \\
\hline $100 \mathrm{mV}$ & $\pm 50 \mathrm{mV}$ \\
\hline
\end{tabular}

Input coupling . DC

Max working voltage

(signal + common mode)

Each input should remain within $\pm 11 \mathrm{~V}$ of ground
Overvoltage protection

\begin{tabular}{|l|l|l|}
\hline \multicolumn{1}{|c|}{ Signal } & Powered On & Powered Off \\
\hline \hline AI $<0 . .15>$ & \pm 42 & \pm 35 \\
\hline AI SENSE & \pm 40 & \pm 25 \\
\hline
\end{tabular}

FIFO buffer size NI DAQCard-6024E ...................2,048 samples (S) NI 6023E, NI PCI-6024E, NI 6025E. $512 \mathrm{~S}$

DMA (PCL/PXI only)

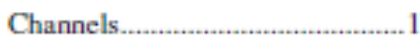

Data sources/destinations............Analog input, analog output, counter/timer 0 , or counter/timer 1

Data transfers Direct memory access (DMA), interrupts, programmed $\mathrm{I} / \mathrm{O}$

DMA modes ${ }^{1}$ Scatter-gather (single transfer, demand transfer)

Configuration memory size ...............512 words

( 1 word $=8$ bits $)$

1 DMA is not available on the NI DAQCard-6024E. 


\section{Laser Power Meter and Detector Head}

\section{Laserstar}

\section{Versatile Laser Power/Energy Meter}

- Two models available: dual and single channel

- Single channel model can be upgraded to dual channel

- Compatible with all 0phir thermopile, pyroelectric, photodiode and RP heads

- Large LCD display

- Backlighting and rechargeable battery

- Screen graphics and statistics (std dev. min, max)

- Analog output

- Built-in RS232 interface

- Log every data point at $>1500 \mathrm{~Hz}$ with pyroelectric heads

- Non-volatile data storage up to 50,000 points

- Laser tuning screen and power log

- Audio sound for laser tuning and low battery

- Statistics package

- GPIB option (IEEE488.1)

- NIST traceable

- CE marked

- Soft keys, menu-driven

The dual channel model enables user to simply plug in any of 0phir's thermal, pyroelectric, photodiode or RP heads and measure two channels independently, or the ratio or difference between them in real time.

Up to 10 data files ( 54,000 points total) can be stored for onboard review or downloading to computer even if Laserstar has been switched off. The built-in RS232 interface and StarCom PC software allow on-line processing of data or processing previously stored data; results are displayed graphically on a PC.

Digital Power Screen

- CW industrial, medical and scientific lasers

- pW to $20 K W$ with appropriate heads

- Can average over selected period Useful for unstable lasers

- Fast response bar graph

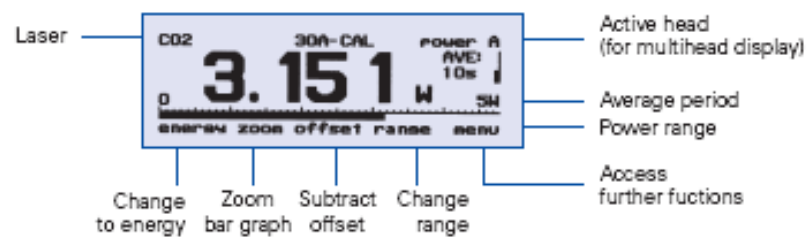

Laser Tuning Screen or Power Log Screen (not shown)

- Maximizing laser power

- User selected time periodand zoom

- Option of audio tune tone

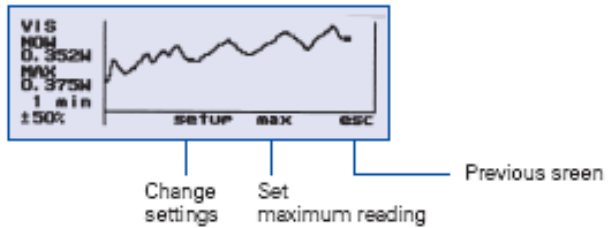


Energy Measurement Screen

- Pyroelectric and thermal heads - single pulse

- Pyroelectric frequency measurement

Energy Log Screen

- Pyroelectric heads

- Thermal heads-successive single pulses

- Continuous scroll

- Energy statistics

Ratio Screen

- Two independent heads

- Measure ratio, sum, difference

- Normalize one head to the other

Data Storage and Transmission

- Non-volatile storage of power and energy logging data

- Store in up to 10 files and transmit to

- $\mathrm{PC}$ using StarCom Windows program provided
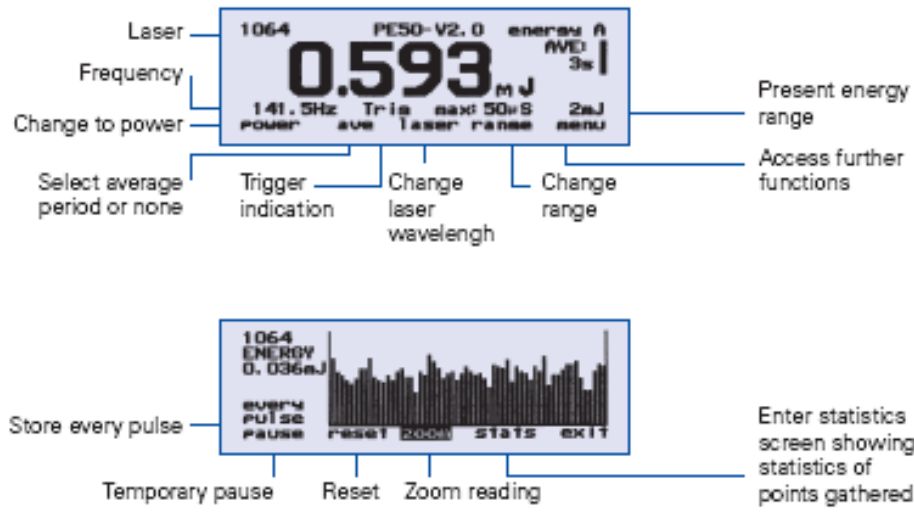

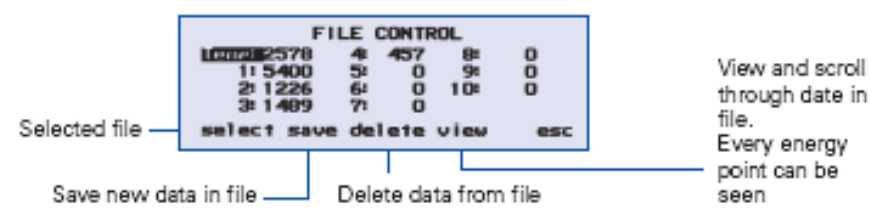

Specifications

\begin{tabular}{|c|c|}
\hline Display & $\begin{array}{l}\text { High legibility } 64 \text { x } 240 \text { pixel graphics supertwist LCD with switchable, electroluminescent backlight which operates from } \\
\text { charger or battery. Large } 17 \mathrm{~mm} \text { digits. Screen refresh } 15 \mathrm{~Hz} \text {. }\end{array}$ \\
\hline Features & $\begin{array}{l}\text { Many screen features including: power with bargraph, energy, average, exposure, frequency, graphs and more. Analog output } \\
1 \text { volt F.S. }\end{array}$ \\
\hline Case & Molded high-impact plastic with swivel display and EMI conductive shielding, to allow use even in proximity to pulsed lasers. \\
\hline Size & Folds to a compact $228 \mathrm{~mm}$ W x $195 \mathrm{~mm} \mathrm{Lx} 54 \mathrm{~mm} \mathrm{H}$. \\
\hline Battery & charge able 18 hours between charges. Charger (included) also functions as AC adapter. \\
\hline Multihead option & Two heads can be connected and measure independently, or the ration, sum or difference of the two can be displayed. \\
\hline Data handling & $\begin{array}{l}\text { Built-in RS232 communic ations at up to } 19200 \text { baud. Non-volatile on-board data storage in } 10 \text { files of up to } 54000 \text { points total. } \\
\text { Data can be viewed on-board or transmitted to PC. }\end{array}$ \\
\hline Head features & $\begin{array}{l}\text { Works with thermal, pyroelectric and photodiode heads. Automatic, continuous, background cancellation with PD300 heads. } \\
\text { Submicrojoule and multikilohertz capability with pyroelectric heads. }\end{array}$ \\
\hline Program features & $\begin{array}{l}\text { User can update calibration information. Preferred startup configuration can be set by user. User can recalibrate power, energy, } \\
\text { response time and zero offset. }\end{array}$ \\
\hline
\end{tabular}

\begin{tabular}{|l|l|l|}
\hline \multicolumn{2}{|c|}{ Ordering Information } \\
\hline IterI & Description & 0 phir P/N \\
\hline Laserstar & $\begin{array}{l}\text { Laserstar single channel universal smart head display for thermal, pyroelectric, photodiode and RP } \\
\text { heads }\end{array}$ & 1 Z01600 \\
\hline Laserstar 2 Channel & Laserstar with dual channel capability including ration and difference measurement & 1 Z01601 \\
\hline Laserstar Hard Case & Hard case 46x35x13cm. For display and up to three heads & 1 Z09101 \\
\hline Laserstar AN adapter & $\begin{array}{l}\text { Laserstar analog output adapter. Plugs into D25 output and provides analog output from BNC plug (1 unit } \\
\text { supplied with Laserstar) }\end{array}$ & 1 1Z11004 \\
\hline Laserstar 2 Ch AN Out & Laserstar AN adapter but for dual channel Laserstar (1 unit supplied with Laserstar) & 1 Z11005 \\
\hline Laserstar 2 Ch Upgrade & Upgrade single channel Laserstar to dual channel version (contact agent for details) & 18121 \\
\hline Laserstar Battery Pack & Replacement battery pack for Laserstar & 1214006 \\
\hline Laserstar RS232 Cable & D25 to D9 cable & 1 Z10032 \\
\hline Laserstar IEEE Option & IEEE GPIB adapter for Laserstar & 18300 \\
\hline Laserstar IEEE Upgrade Kit & Upgrade for existing Laserstar & 18301 \\
\hline
\end{tabular}




\section{PE50BB-DIF/PE50DIF-ER}

\section{Pulse Energy Measurements $60 \mu \mathrm{J}$ to $40 \mathrm{~J}$, up to $400 \mathrm{~Hz}$}

Recommended Use: PE50BB-DIF: High energy YAG lasers, general

PE50DIF-ER: High rep rate high energy lasers, Erbium lasers

Special Features: Removable diffuser for high damage threshold

\begin{tabular}{|c|c|c|c|c|c|c|c|c|}
\hline \multirow[t]{2}{*}{ Specifications } & \multicolumn{4}{|l|}{ PE50BB-DIF } & \multicolumn{4}{|l|}{ PE50DIF-ER } \\
\hline & \multicolumn{2}{|l|}{ Diffuser out } & \multicolumn{2}{|l|}{ Diffuser in } & \multicolumn{2}{|l|}{ Diffuser out } & \multicolumn{2}{|l|}{ Diffuser in } \\
\hline Aperture: & \multicolumn{2}{|l|}{$\varnothing 46 \mathrm{~mm}$} & \multicolumn{2}{|l|}{$\varnothing 33 \mathrm{~mm}$} & \multicolumn{2}{|l|}{$\varnothing 46 \mathrm{~mm}$} & \multicolumn{2}{|l|}{$\varnothing 33 \mathrm{~mm}$} \\
\hline Spectral Response: & \multicolumn{2}{|l|}{$0.19-20 \mu \mathrm{m}$} & \multicolumn{2}{|l|}{$0.4-2.5 \mu \mathrm{m}$} & \multicolumn{2}{|l|}{$0.19-3 \mu \mathrm{m}$} & \multicolumn{2}{|l|}{$0.4-3 \mu \mathrm{m}$} \\
\hline Surface Reflectivity: & \multicolumn{2}{|l|}{$5 \%$} & \multicolumn{2}{|l|}{$15 \%$} & \multicolumn{2}{|l|}{$50 \%$} & \multicolumn{2}{|l|}{$15 \%$} \\
\hline Calibration Accuracy: & \multicolumn{2}{|l|}{ $\pm 3 \%$} & \multicolumn{2}{|l|}{ $\pm 3 \%$} & \multicolumn{2}{|l|}{ $\pm 3 \%$} & \multicolumn{2}{|l|}{ $\pm 3 \%$} \\
\hline \multicolumn{9}{|l|}{ Additional Error with Wavelength: } \\
\hline $248 \mathrm{~nm}$ & \multicolumn{2}{|l|}{ $\pm 2 \%$} & \multicolumn{2}{|l|}{ NA. } & NA. & & N.A. & \\
\hline $400-800 \mathrm{~nm}$ & $\pm 2 \%$ & & Ser note a & & $\pm 2 \%$ & & See note b & \\
\hline $1064 \mathrm{~nm}$ & 0 & & 0 & & 0 & & 0 & \\
\hline $1.5-2.5 \mu \mathrm{m}$ & $\pm 2 \%$ & & Se日 note b & & NA. & & See note b & \\
\hline $2.1 \mu \mathrm{m}$ & $\pm 2 \%$ & & 0 & & NA. & & 0 & \\
\hline $2.94 \mu \mathrm{m}$ & $\pm 2 \%$ & & NA. & & NA. & & 0 & \\
\hline $10.6 \mu \mathrm{m}$ & $\pm 5 \%$ & & NA. & & NA. & & N.A. & \\
\hline Damage Threshold: & & & & & & & & \\
\hline$<100 \mathrm{~ns}$ & $0.3 \mathrm{~J} / \mathrm{cm}^{2}$ & & $3 \mathrm{~J} / \mathrm{cm}^{2}$ & & $0.1 \mathrm{~J} / \mathrm{cm}^{2}$ & & $1.5 \mathrm{~J} / \mathrm{cm}^{2}$ & \\
\hline $1 \mu \mathrm{s}$ & $0.3 \mathrm{~J} / \mathrm{cm}^{2}$ & & $3 \mathrm{~J} / \mathrm{cm}^{2}$ & & $0.2 \mathrm{~J} / \mathrm{cm}^{2}$ & & $3 \mathrm{~J} / \mathrm{cm}^{2}$ & \\
\hline $300 \mu 8$ & $1 \mathrm{~J} / \mathrm{cm}^{2}$ & & $10 \mathrm{~J} / \mathrm{cm}^{2}$ & & $4 \mathrm{~J} / \mathrm{cm}^{2}$ & & $40 \mathrm{~d} / \mathrm{cm}^{2}$ & \\
\hline Linearity: & $\pm 2 \%$ for $>10$ & of full scale & $\pm 2 \%$ for $>10$ & 6 of full scale & $\pm 2 \%$ for $>10$ & of full scale & $\begin{array}{l} \pm 2 \% \text { for }>10 \\
\text { scale }\end{array}$ & $\%$ of full \\
\hline Maximum Average Power: & $15 \mathrm{~W}$ & & $40 \mathrm{~W}$ & & $20 \mathrm{~W}$ & & $40 \mathrm{~W}$ & \\
\hline Max Ave Power Density: & $10 \mathrm{~W} / \mathrm{cm}^{2}$ & & $500 \mathrm{~W} / \mathrm{cm}^{2}$ & & $10 \mathrm{~W} / \mathrm{cm}^{2}$ & & $500 \mathrm{~W} / \mathrm{cm}^{2}$ & \\
\hline Max Pulse Width Setting: & Short & Long & Short & Long & Short & Long & Short & Long \\
\hline Maximum Pulse Width: & $3 \mathrm{~ms}$ & $10 \mathrm{~ms}$ & $3 \mathrm{~ms}$ & $10 \mathrm{~ms}$ & $200 \mu 18$ & $1 \mathrm{~ms}$ & $200 \mu 8$ & $1 \mathrm{~ms}$ \\
\hline Maximum Pulse Rate: & $40 \mathrm{~Hz}$ & $10 \mathrm{~Hz}$ & $40 \mathrm{~Hz}$ & $10 \mathrm{~Hz}$ & $400 \mathrm{~Hz}$ & $200 \mathrm{~Hz}$ & $400 \mathrm{~Hz}$ & $200 \mathrm{~Hz}$ \\
\hline Energy Scales: & $10 \mathrm{~J}$ to $2 \mathrm{~mJ}$ & $10 \mathrm{~J}$ to $20 \mathrm{~mJ}$ & $40 \mathrm{~J}$ to $8 \mathrm{~mJ}$ & $40 \mathrm{~J}$ to $80 \mathrm{~mJ}$ & $10 \mathrm{~J}$ to $2 \mathrm{~mJ}$ & $10 \mathrm{~J}$ to $2 \mathrm{~mJ}$ & $30 \mathrm{~J}$ to $6 \mathrm{~mJ}$ & $30 \mathrm{~J}$ to $6 \mathrm{~mJ}$ \\
\hline Lowest Measurable Energy: & $100 \mu \mathrm{J}$ & $1 \mathrm{~mJ}$ & $500 \mu \mathrm{J}$ & $5 \mathrm{~mJ}$ & $60 \mu \mathrm{J}$ & $100 \mu \mathrm{J}$ & $300 \mu \mathrm{J}$ & $0.5 \mathrm{~mJ}$ \\
\hline Noise on Lowest Range: & $15 \mu \mathrm{J}$ & $40 \mu \mathrm{J}$ & $80 \mu \mathrm{J}$ & $200 \mu \mathrm{J}$ & $5 \mu \mathrm{J}$ & $10 \mu \mathrm{J}$ & $30 \mu \mathrm{J}$ & $50 \mu \mathrm{J}$ \\
\hline Additional Error with Frequency: & $\pm 1 \%$ & $\pm 1 \%$ & $\pm 1 \%$ & $\pm 1 \%$ & $\pm 1 \%$ & $\pm 1 \%$ & $\pm 1 \%$ & $\pm 1 \%$ \\
\hline Cooling Corvection & & & & & & & & \\
\hline $\begin{array}{l}\text { Notes: a: Calkrated for } 532 \mathrm{~nm} \\
\text { tr: With diffuser installed, head }\end{array}$ & calibrated at t & hese wavelength & Ut provisisn is m & de for user calib & & & & \\
\hline
\end{tabular}

PE50BR-DIF/PE50DIF-ER

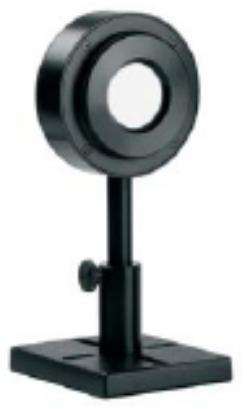

DIFFUSER in

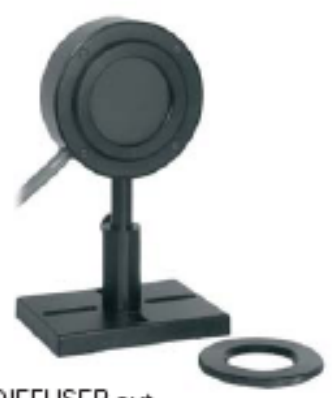

DIFFUSER out

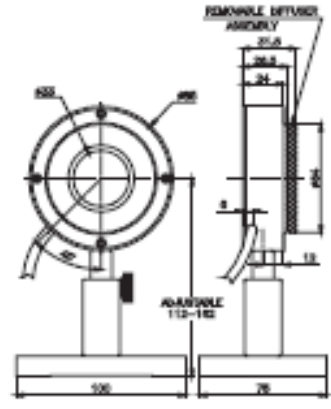

Ordering information

\begin{tabular}{|l|l|l|}
\hline Item & Description & 0 phir P/N \\
\hline PE50BB-DIF-V2 & $33 / 46 \mathrm{~mm}$ aperture pyroelectric energy meter with broadband absorber and removable diffuser & $1 Z 02866$ \\
\hline PE50DIF-ER-V2 & $33 / 46 \mathrm{~mm}$ aperture pyroelectric energy meter with metallic absorber and removable diffuser & 1202867 \\
\hline
\end{tabular}




\section{High Speed Photodiode Detector: DET110}

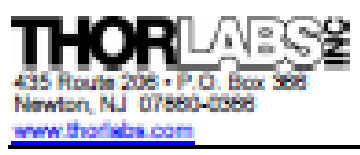

Pre $973-509-727$

FAx $2073-300-3600$

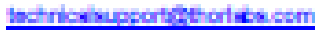

\section{DET110 - HIGH-SPEED SILICON DETECTOR}

\section{DESCRIPTION:}

Thoriaba' DET110 is a ready-to-use high-apeed photo detector. The unit comes complete with a photodlode and Intema: $12 \mathrm{~V}$ blas battery enclosed in a ruggedlzed aluminum housing. The head includes a removabie $1^{*}$ optical coupler (8M1T1), providing easy mounting of ND ni:ers; spectral fliters and other Thorlabs 1" atackable lens mount acceasoriea. Also avalable are flber adapters (BMA, FC and BT atyle). An $\neq 9-32$ tapped hole la provided on the base of the housing to mount the detector drectly to a Thoriabs' poaltioning device (1/2* post holder, mounting plates, etc.).

\section{SPECIFICATIONS:}

\begin{tabular}{|c|c|c|c|}
\hline Deteotor: & Slicon PIN & Houcing: & Black Anodzed Aluminum \\
\hline 8peotral Recponce: & $350-1100 \mathrm{~nm}$ & stze: & $\$ 1.43^{\circ} \times 1.67^{*}$ \\
\hline Poak Wavolength: & $960 \mathrm{~nm}+i-50 \mathrm{~nm}$ & Output: & BNC, DC-Coupled \\
\hline $\begin{array}{l}\text { Riceifall Time': } \\
\text { Dlode Capaoltanes: }\end{array}$ & $\begin{array}{l}20 n s \\
20 p F\end{array}$ & $\begin{array}{l}\text { Blac: } \\
\text { Mounting: }\end{array}$ & $\begin{array}{l}\text { 12V Battery (Type A23) } \\
\text { 8-32 (M4) Tapped Hole }\end{array}$ \\
\hline NEP: & $1.2 \times 10^{-14} \mathrm{~W} / \mathrm{V} / \mathrm{Z}$ & $\begin{array}{l}\text { Dlodo } \\
\text { sooket: }\end{array}$ & TO-5, Anode Marked \\
\hline Dark Current: & $10 n A$ & $\begin{array}{l}\text { Damage } \\
\text { Threchold: }\end{array}$ & $100 \mathrm{~m} W \mathrm{cW}$ \\
\hline Aotlvo Ares: & $\begin{array}{l}13 \mathrm{~mm}^{2} \\
3.6 \mathrm{~mm} \times 3.6 \mathrm{~mm} \text { square }\end{array}$ & & $0.5 \mathrm{~J} / \mathrm{cm}^{2}$ (10ns pulse) \\
\hline
\end{tabular}

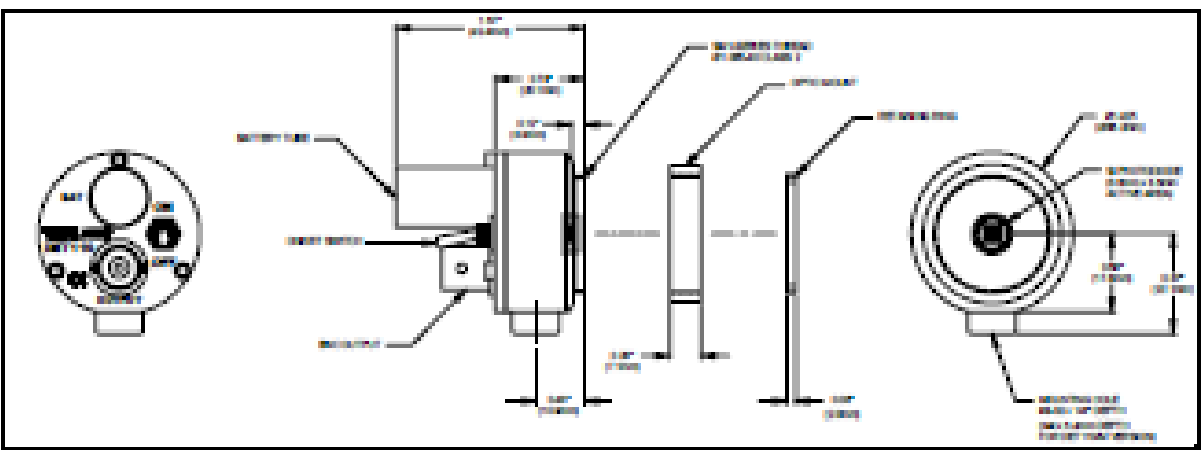

Floure 1. - Moohanlosi Dimenclone

\section{OPERATION:}

Thoriaba DET series are ideal for measuring both pulsed and CW light sourcea. The DET110 includes a reversed-blsaed PIN photo dode, blas battery, and ON/OFF swltch packaged in a ruggedzed housing. The aNC output algnal ls the drect photocument out of the photo dlode anode and is a function of the incident light power and waveiength. The Spectral Responsivity, $भ(\lambda)$, can be obtained from Figure 2 to eatimate the amount of photocurrent to expect. Most users will wish to convert this photocurrent to a voltage for viewing on an osclloscope or DVM. Thls la accompllahed by addling an extemal lood realatance, Rlow. The output voltage is derived as:

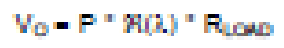

The bandwidth, faw, and the rise-time reaponse, tos are determined from the dlode capacitance, $\mathrm{C}_{4}$ and the losd realstance, Row as shown below:

$$
\begin{gathered}
\text { fow }=1 /\left(2^{*} \pi^{*} R_{\text {LOW }}{ }^{*} C_{2}\right) \\
\text { to }=0.35 / \text { fow }
\end{gathered}
$$


For maximum bandwleth, we recommend using a sos coax cable with a son terminating realator at the end of the coax. This will also minimize ringing by matching the coax with its characteriatic impedance. If bandwidth is not important, you may increase the amount of voltage for a given input light by increasing the R Low up to a maximum of $10 \mathrm{KS}$.

Note: The deteotor hac an AC path to ground oven with the ewitoh In the OFF pocition. It ic normal to cee an output recponce to an AC clonal with the ewitoh In thic ctate. However, beosuce the deteotor IC unblaced, operation In thic mode le not reoommended.

Flgure 2 - Typloal DET110 3peotral Recponelvity Curvo
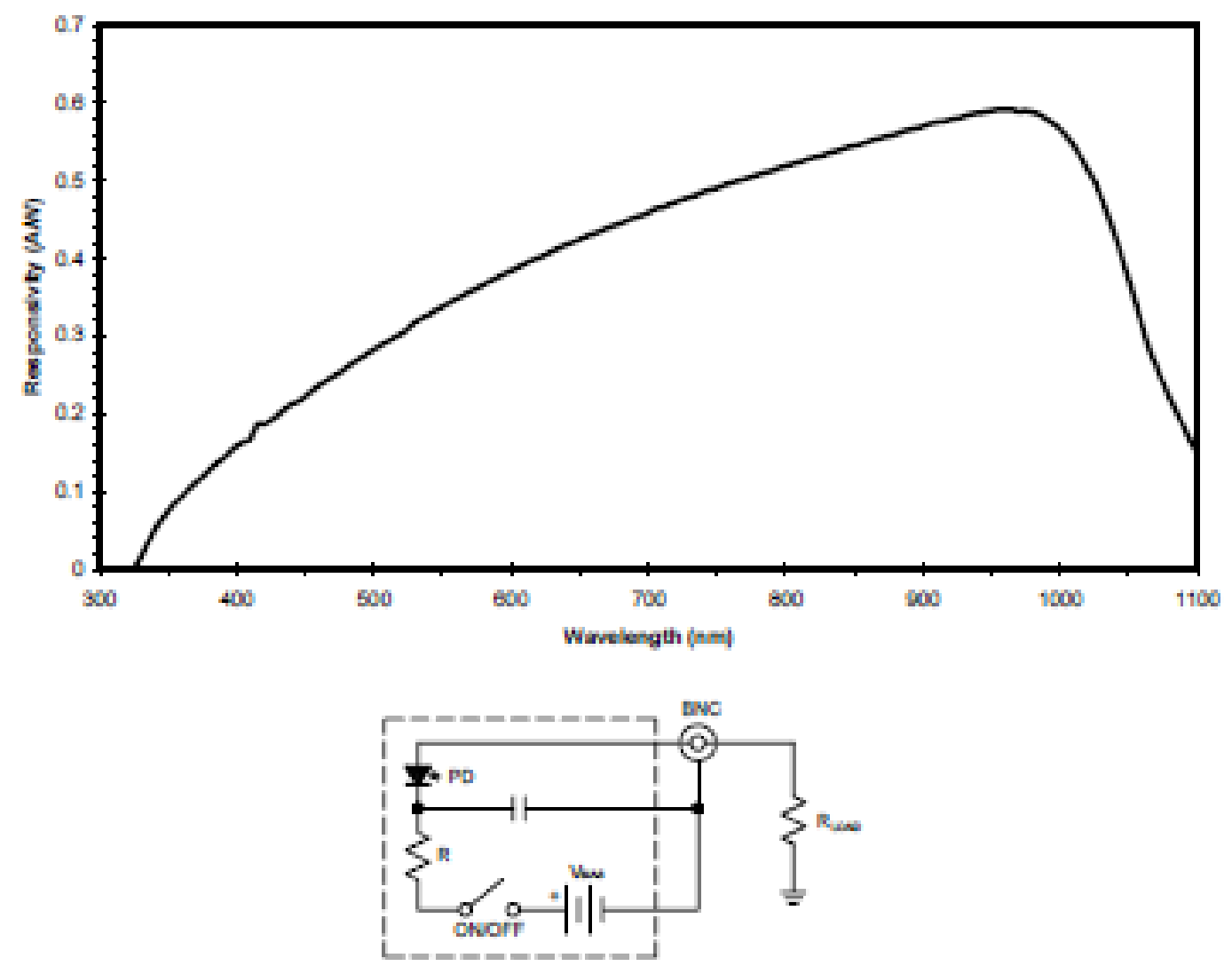

Flgure 3 - Clroult Blook. Dlagram

\section{FIBER ADAPTERS AND OTHER ACCESSORIES}

Thoriaba sella a number of accessories that are compatible with the $1^{\circ}$ thread on the DET housing including FC, SMA, and BT nber adapters, stackable lena tubes for mounting optcs, and cage assemblles that allow the DET to be Incorporated into elaborate 3-D optical asaemblea.

Caution: The DET110 was deslgned to allow maximum accessbility to the photo detector by having the front surface of the dlode extend outside of the DET housing. When using flber adspters, make sure that the flber ferrule does not crash Into the detector. Fallure to do 20 may cause damage to the dlode and / or the flber. An easy way to accompllah this is to Install a aM1RR retaining ring (Included with the DET110) inalde the $1^{\circ}$ threaded coupler before inatalling the flber adspter.

Also avalable are ina sI detectors, lnGaAs detectors, and a complete line of ampified detectors.

\section{MAINTAINING THE DET110}

There are no serviceabie parta in the DET110 optical head or power supply. The houaing may be cleaned by wiping with a sot, damp cloth. The window of the detector ahould onty be cleaned using optical grade wipes. If you suspect a problem with your DET110 please call Thorlabs and technical support will be happy to assiat you.

2199-001 Rev D 8/15/2005 


\section{Oscilloscope: TDS 2014}
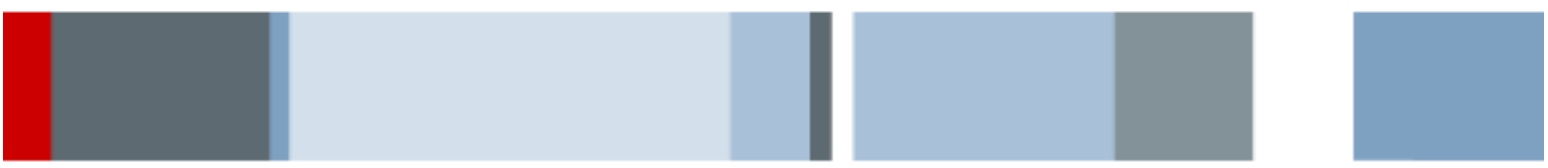

\section{Digital Storage Oscilloscopes}

\section{TDS2000C Series Data Sheet}

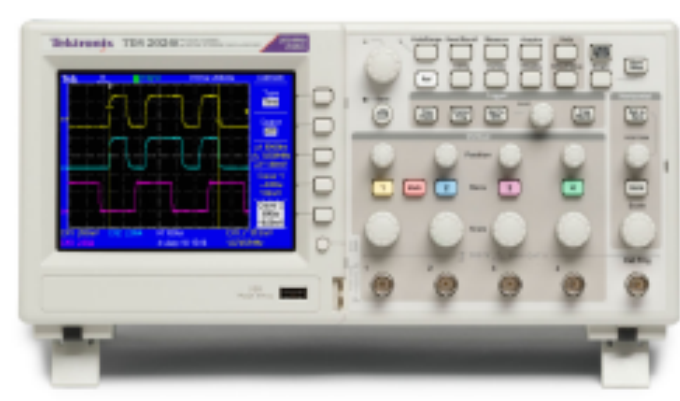

\section{Ease-of-Use Features}

- 16 Automated Measurements, and FFT Analysis for Simplifed Waveform Analysis

- Buit-in Waveform Limit Testing

- Automated, Extended Data Logging Feature

- Autoset and Signal Auto-ranging

- Buit-in Context-sensitive Help

- Probe Check Wizaro

- Multiplelanguage User Interface

- 5.7 in. (144 mm) Active TFT Color Display

Features \& Benefits

- Small Footprint and Lightweight - Only 4.9 in. (124 mm) Deep and $4.4 \mathrm{lb}$. (2 kg)

Key Performance Specifications

Connectivity

- $200 \mathrm{MHz}, 100 \mathrm{MHz}, 70 \mathrm{MHz}, 50 \mathrm{MHz}$ Bandaidth Models

- 2- and 4-channel Models

- Up to 2 GS/s Sample Rate on All Channels

- $2.5 \mathrm{k}$ point Recond Length on All Channels

- Advanced Triggers including Pulse Width Trigger and Line-selectable video Trigger

- USB 2.0 Host Port on the Front Panel for Quick and Easy Data Storage

- USB 2.0 Device Port on Rear Panel for Easy Connection to a PC or Direct Printing to a Picteridge ${ }^{*}$-compatible Printer

- Includes National Instruments LabVIEW SignalExpress N TE Limited Edition and Tektronix OpenChoice* Software for Connecting Your Bench

Lifetime Warranty ${ }^{* 1}$

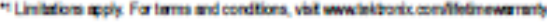

\section{Tektronix}


$\underline{\text { Xantrex DC Power Supply }}$

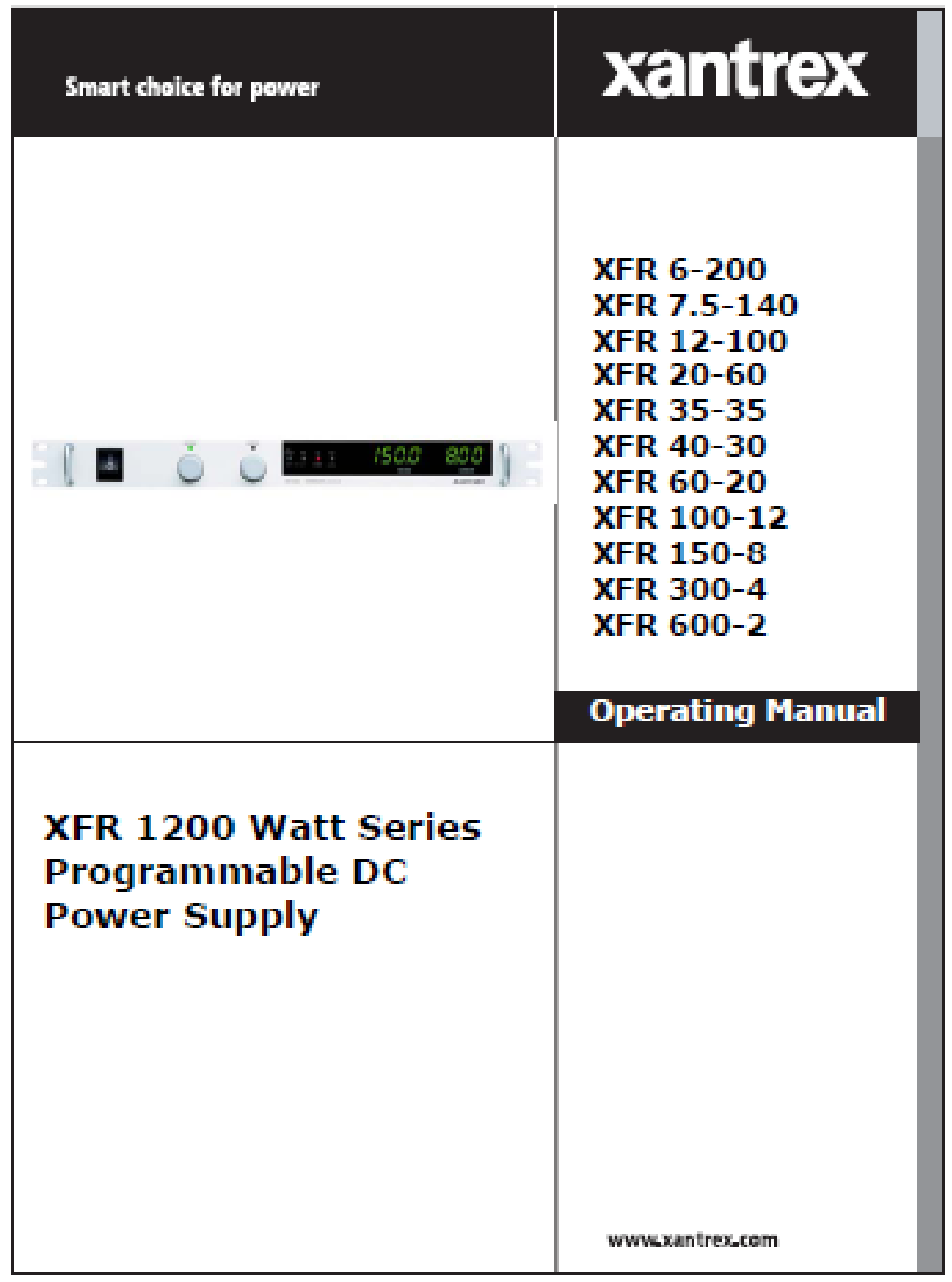




\title{
Section 1. Features and Specifications
}

\author{
Description
}

This saiw of power suplias proides low-poine, frocivaly regulated, waible DC

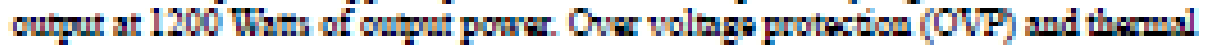

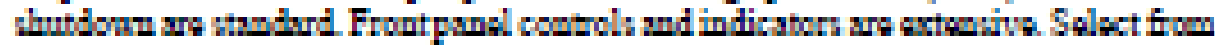

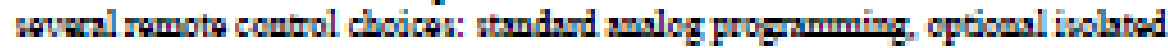
Frogramming or resibsek, and optionsl GPI programming or KS-232 control. Rawote monitoring of output volmge and arrent in a smidrd fatura. Uhe th: pown suply athar os your banch or in a randerd $19 \mathrm{~m}$ ( $483 \mathrm{~mm}$ ) rack the porw

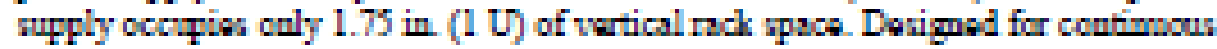

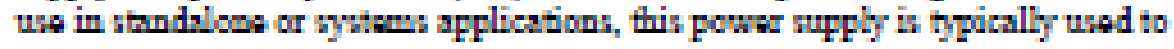

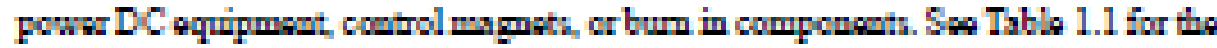
lit of arilable modals.

Table 1.1 Awalable Voltage and Cument Rianges

\begin{tabular}{|c|c|c|}
\hline Moda & Wotspe Fanq: & Current Fung \\
\hline 6200 & DEY & $1-200 \mathrm{~A}$ \\
\hline $7 \leq-140$ & 7-7.5 V & I-14] A \\
\hline $12-120$ & $5-12 Y$ & D-1D] A \\
\hline MeO & I-20 Y & I-6D A \\
\hline $35-35$ & $0-35 \mathrm{~V}$ & प-35A \\
\hline $40-20$ & $0-40 \mathrm{Y}$ & {$[-30 \mathrm{~A}$} \\
\hline $60-20$ & DAD Y & I-20 $\mathrm{A}$ \\
\hline $100-12$ & D-100 V & {$[-12 A$} \\
\hline $150-8$ & $0-150 \mathrm{~V}$ & I-GA \\
\hline $300-4$ & O-200 V & D-4A \\
\hline $600-2$ & DADL V & IDA \\
\hline
\end{tabular}




\section{Chiller: Neslab RTE-111}

\section{Refrigerated Bath/Circulator} Specifications

\begin{tabular}{|c|c|}
\hline MODEL & RTE-111 ..., \\
\hline \multicolumn{2}{|l|}{ TEUPERATURE RAMGE } \\
\hline Analog: & $-25^{\circ} \mathrm{C}$ to $+100^{\circ} \mathrm{C}$ \\
\hline $\begin{array}{l}\text { DigtalMaroporocesoor: } \\
50 \text { itz Model: }\end{array}$ & $-25^{\circ} \mathrm{C}$ to $+150^{\circ} \mathrm{C}$ \\
\hline Analog: & $-18^{\circ} \mathrm{C}$ to $+100^{*} \mathrm{C}$ \\
\hline Diglas/Meroprocesoor: & $-18^{\circ} \mathrm{C}$ to $+150^{\circ} \mathrm{C}$ \\
\hline TEUPERATURE STABLTTY & $\pm 0.01^{\circ} \mathrm{C}$, Ansiog $=0.1^{\circ} \mathrm{C}$ \\
\hline COCUNO CAPACITY & 500 watts at $20^{\circ} \mathrm{C}$ \\
\hline $50 \mathrm{~Hz}$ Models & 375 Watts at $20^{\circ} \mathrm{C}$ \\
\hline PUMPING CAPACITY & $\begin{array}{l}15 \text { Lm at of head } \\
\text { Max Head } 16^{\prime}(49 M)\end{array}$ \\
\hline HEATER & 800 watt \\
\hline WOFK AREA $(\mathrm{L} \times W \times \mathrm{D})$ in. & $\begin{array}{c}4 \times 4 \times 8 \times 6 \\
12.1 \times 20.3 \times 15.2\end{array}$ \\
\hline BATH WOLUME GalonaLIter: & 1.977 .0 \\
\hline $\begin{array}{r}\text { DIUENSIONS }(\mathrm{H} \times W \times \mathrm{D}) \mathrm{In} . \\
\mathrm{Cm} .\end{array}$ & $\begin{array}{l}25 \times 10^{8 / 18} \times 15^{78} \\
63.5 \times 25.2 \times 40.3\end{array}$ \\
\hline $\begin{array}{l}\text { POWER REQUIREMENTI3 } \\
50 \mathrm{~Hz} \text { Modei: }\end{array}$ & $\begin{array}{c}115 \mathrm{~V}, 60 \mathrm{~Hz}, 12 \mathrm{Amps} \\
220-240 \mathrm{~V}, 50 \mathrm{~Hz}, 7.5 \mathrm{Amps}\end{array}$ \\
\hline SHPPINO WEIGHT & B5 Lbe/39 Kge \\
\hline \multicolumn{2}{|c|}{ 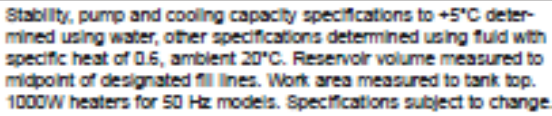 } \\
\hline Cooling Cap & \\
\hline
\end{tabular}

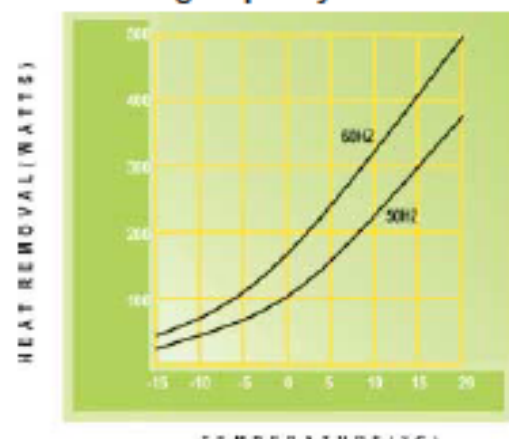

TE UPERATURE|⿰冫欠

\section{Pumping Capacity}

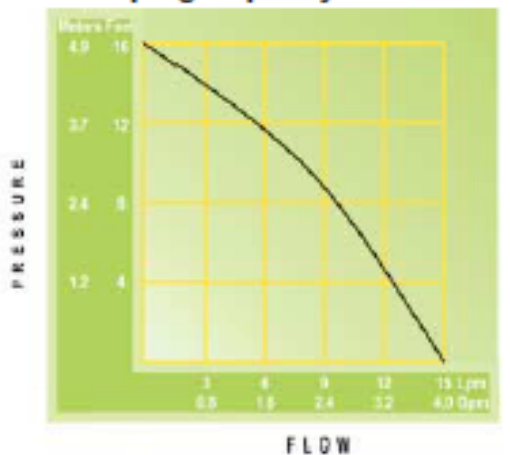

For sales and service information call 800/4NESLAB or 800/258-0830

\section{FEATURES}

Versatile combination force/suction pump

Rapid cool down with no waiting

Choose analog, digital or remote microprocessor controller

Built in handles for safe leverage

High temp/ low liquid safety

Designed for UL, CSA, CE, and IEC

Digital Controller Features a bright green LED display and allows the user to select variables for temperature display. Both the microprocessor and digital controllers offer this feanure

Direct Interface
A direct RS-232 port allows for conmputer control.

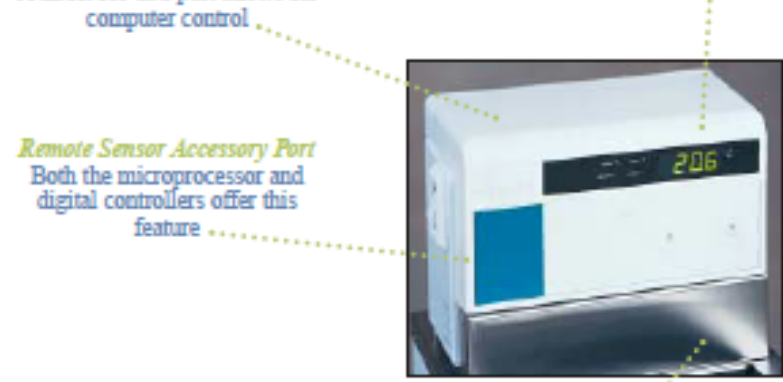

Propertional Control The digital controller also features proportional control with auto load reset for rapid temperature stabilization under changing heatloads 


\section{Fiber Coupled Diode Laser}

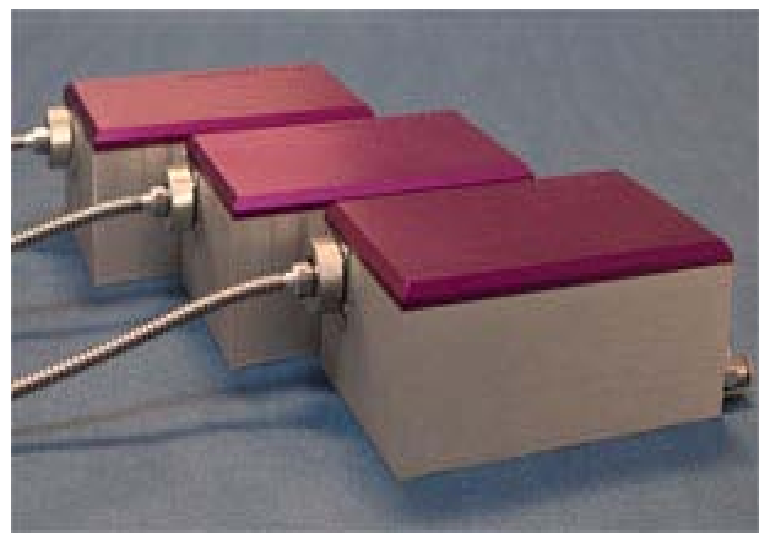

Apollo Instruments' fiber-coupled laser diodes offer brightness and high power with unprecedented beam ty. These compact devices deliver up to $10 \mathrm{~kW}$ gh a fiber assembly with core diameter ranging from $1 \mathrm{~mm}$. These devices can be offered in various gurations. Fiber-coupled modules that deliver up to atts are conductively cooled and ready for mounting heat sink. Devices with higher power are normally : cooled. Apollo's water-cooled devices are robust. 2 is no need to use de-ion water or ultra-filtration. The -coupled laser diodes provide ideal beam sources for $\underline{m}$ integration and offer the simplest and easiest way liver high-power laser light

to a variety of applications. The devices are cost-effective pump sources for laser pumping (such as fiber laser pumping). They can also be excellent beam sources for material processing and medical applications. Due to the high brightness, the devices have been shown to be effective in direct laser applications such as metal cutting, marking, soldering, welding and silicon wafer treatment. Together with a focusing lens, some laser modules can easily achieve a beam spot with a power density over $4 \mathrm{MW} / \mathrm{cm}^{2}$. Models are now available in a variety of wavelengths ranging from $780 \mathrm{~nm}-1530 \mathrm{~nm}$. Although the standard modules are built with a single wavelength, multiple wavelengths can be combined. One of the unique characteristics of Apollo Instruments' fibercoupled devices is that all the output is emitted from the fiber core. There is no cladding mode.

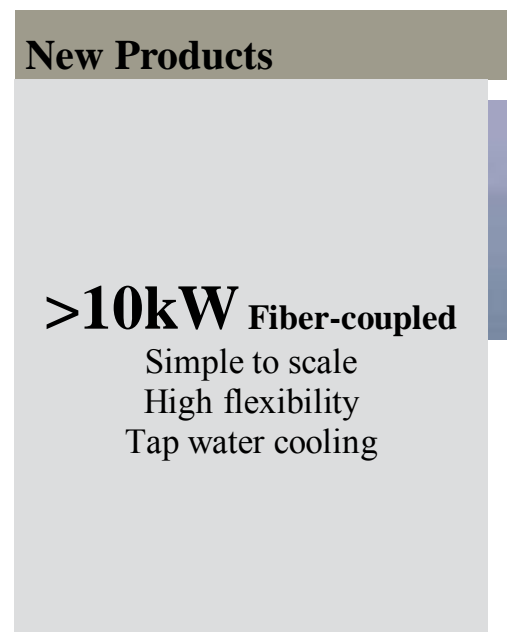

Laser Controler: Northrop Grumman- eDrive

Ready for splicing

Tap water-cooled

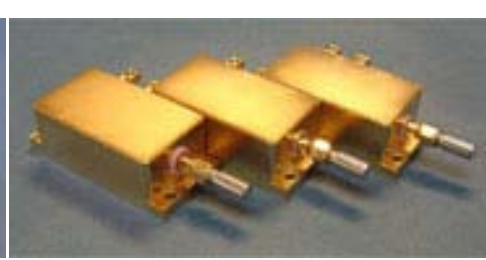

30W Fiber-Coupled

Solid-state laser pump Cost effective

High reliability / Long lifetime

Compact

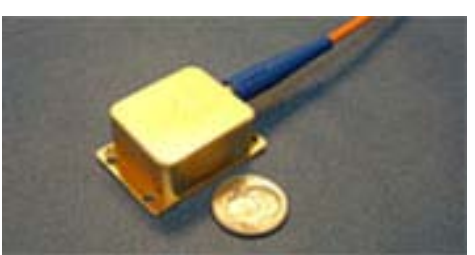

20W Fiber-Coupled

Mini-series

Cost effective

Passive cooling High brightness 


\section{ELECTRONICS \& SUPPORT EQUIPMENT}

\section{eDrive $^{\mathrm{TM}}$}

ED2U SERIES DRIVER

LASER SYSTEM \& LASER DIODE DRIVER

\section{FEATURES AND BEMEFITS}

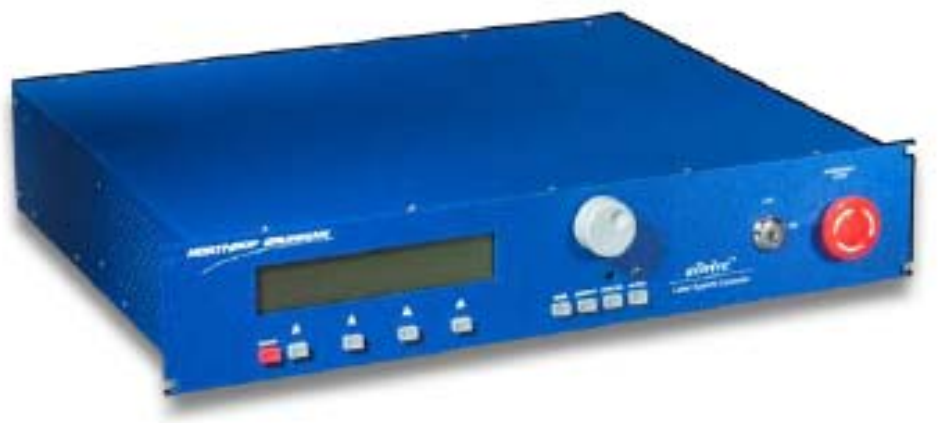

- Operates in both CW and QCW modes

- 0-350V compliance voltage range

$-100 \mathrm{~A}$ or $300 \mathrm{~A}$ pulsed versions available

- Water or Air cooled versions available

- CE marked versions available

$-19^{*}$ rack mountable

- Coolant and safety interlocks

- Labriew Compatible:

RS232 and RS485 interface

Configured to run diodes in either CW or QCW modes, the eDrive controller is an excellent tool for performing all critical operations for laser diode arrays in one box. With drive ratings of 70A CW and 300A OCW $1 \leq 5 \%$ Duty cycle (water cooled version), the eDrive is capable of performing laser system functions such as shutter control, system interlodks, and Q-switching. The user has ultimate control with limit features for current and duty cycle, which allows the eDrive to protect the laser diode arrays.

All eDrives are equipped with multiple control options including RS232 and RS485 interface and are Labview ${ }^{\mathrm{MM}}$ compatble. Whether you are in production or working in a research and development environment, the eDrives versatility will support your requirements well into the future. If you have ary further questions, contact one of our sales representatives. We would be pleased to work with you to configure a controller to fit your needs.

"Labvaw" is the property of Natoral instruments Corporaten. 


\begin{tabular}{|c|c|c|c|c|}
\hline & \multicolumn{2}{|c|}{ Arr Coolsed } & \multicolumn{2}{|c|}{ Whter Cooliad } \\
\hline Ampiliude: & GN. & oon & $D W$ & $\sigma W$ \\
\hline Oulput Currant & $0.50 \mathrm{~A}$ & $0.300 \mathrm{~A} *$ & $0.70 \mathrm{~A}$ & $0.300 \mathrm{~A} *$ \\
\hline Desploy Pesolution & $100 \mathrm{~mA}$ & $100 \mathrm{~mA}$ & $100 \mathrm{~mA}$ & $100 \mathrm{~mA}$ \\
\hline Acouncy & \pm 29 & \pm 29 & \pm 29 & \pm 28 \\
\hline Notera & $\angle 50 \mathrm{mApp}$ & $\angle 50$ mApp & $\angle 50 \mathrm{mApp}$ & $<50 \mathrm{mApp}$ \\
\hline
\end{tabular}

*Sea dart an poge 3 for auty oyda ratings

\begin{tabular}{|c|c|}
\hline \multicolumn{2}{|l|}{ Pulas Rallex } \\
\hline \multicolumn{2}{|l|}{ Plange } \\
\hline Desplay Pesolution & $(\mathrm{H} H$ \\
\hline & $10 \mathrm{Hs}$ \\
\hline & $1700 \mathrm{Hes}$ \\
\hline \multicolumn{2}{|l|}{ Acomay } \\
\hline \multicolumn{2}{|l|}{ Pulas Woth: } \\
\hline \multicolumn{2}{|l|}{ Flanga } \\
\hline \multicolumn{2}{|l|}{ Display Pascolition } \\
\hline Tanstion Time & \\
\hline
\end{tabular}

Thlogar in:

Iypo

Sgrai Input

Mnimum Widt

input impodsnce

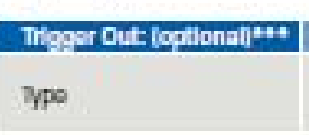

Charpcteristacs

**Non standard avalabla upon requeat

Complanco Voltagx

Flange

Display Fesolution

Acouncy

Current Moniltor.

7/pe

Accurnoy

Interlade:

Opan Crcut Voltage

Short Ciroat Cument

Type

Cencral:

Cperding Terrporature

Pown Input

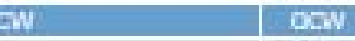

D. $50 \mathrm{dHz}$

D- $100 \mathrm{~Hz}$

$100 \mathrm{~Hz}-1 \mathrm{kHz}$

$1 \mathrm{kHz}-50 \mathrm{kHz}$

$\pm 29$

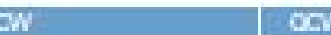

$-$

\section{CoN}

10 j5- $100 \mathrm{~ms}$

$100 \mathrm{~ns}$
5 ps Typical (1000 A)

40 E 7 ppacal $1000 \mathrm{~A}$

\section{Dah}

Postive Edgo Irigger

TTL or 5V CMOS

$50 \mu \mathrm{s}$

$50 \mathrm{a}$

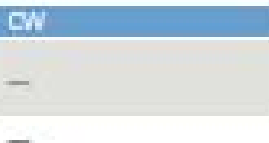

EW

DEM

User configuabio for ED Q swriched oporation

Th of 5N CMOS

\section{CW}

-

-

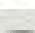

$-$

$-$

$-$

WW

-

-

-

BW

-

$-$

$-$

$-$

EV

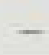

-
COW

0. $50 \mathrm{kHz}$

$0.100 \mathrm{~Hz}$

$100 \mathrm{~Hz}-1 \mathrm{kHz}$

$1 \mathrm{kHz}-50 \mathrm{kHz}$

$\pm 28$

\section{DOW}

$10 \mathrm{ps}-100 \mathrm{~ms}$

100 re

5 js ippaal noo Al

40 us Typical (300 A)

\section{DEW}

Positrie Eoge Tingor

TTL or 5 V CMNS

50 HE

500

\section{and}

Usar contgunblo for EO Q swhthed operatun

TTL of $5 \mathrm{~V}$ CMOS

$0 \mathrm{~W}$
$0.350 \mathrm{~V}$
$0.1 \mathrm{~V}$
$\pm 2 \mathrm{~F}$

EW

to A O.5

$\pm 29$

EN

5 VDC nomral

I mA naminal

Swith contact dosure

or TIL or 5 V CMOS

compatible

\section{rav}

$0-350 \mathrm{~V}$

a.1V

$\pm 27$
$\mathrm{DW}$

0 - $40 \mathrm{C}$, non condensing

$100-240 \mathrm{VAC}, 50,60 \mathrm{Ht}$,

$4 A \max$

\section{ach}

20 AN O.5V 10.15V for

high current modola)

$\pm 28$

and

5VDC namral

1 mA norrinal

Switch contect dosure

or TIL or 5 V CMOS

competibis

\section{$\mathrm{DEW}$}

0. $40{ }^{\circ} \mathrm{C}$, non condensing

100 - 240 vAC, 50,60 Ht,

$4 A \max$

\section{CW}

Q-40'C, non condorsing

100 - 260 VAC, $50 / 60 \mathrm{~Hz}$,

4 A $\max$
CDV

0. $350 \mathrm{~V}$

$0.1 \mathrm{~V}$

$\pm 296$

\section{aEx}

20 AN 0.5V 10-15V for

high curnont modole:

$\pm 2 \%$

\section{IDW.}

5VDC noming

$1 \mathrm{~mA}$ norring

Switch contact dosurs

or TIL \& 5 V CMOS compaticio

\section{CEW}

0 - $40^{\circ} \mathrm{C}$, non condersng

$100-240$ WaC, $50 / 50 \mathrm{~Hz}$,

$4 A \max$ 


\section{Nd:YAG Laser Rod}

Sellmeier Equation:

$n^{2}=A+\frac{B \lambda^{2}}{\lambda^{2}-C}+\frac{D \lambda^{2}}{\lambda^{2}-E}$

where

$$
\begin{aligned}
& \mathrm{A}=1 \\
& \mathrm{~B}=22719 \\
& \mathrm{C}=0.01142 \\
& \mathrm{D}=0 \\
& \mathrm{E}=0
\end{aligned}
$$

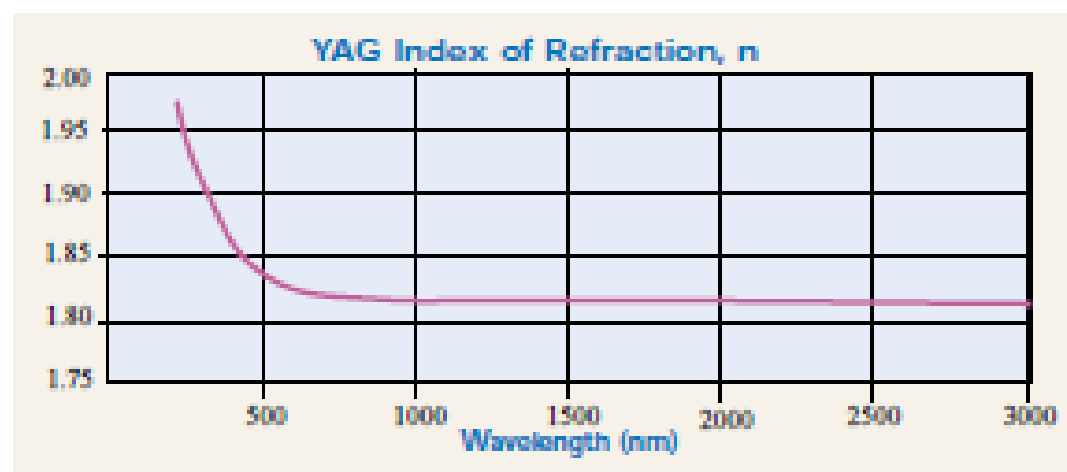

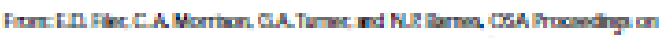

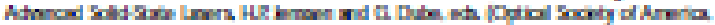

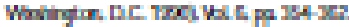

YAG index of retraction and reflectance

$\begin{array}{rcc}\text { Whodisngth (mm) } & \mathrm{n} & \mathrm{R} \\ 265 & 1.9278 & 0.1004 \\ 354 & 1.8725 & 0.0923 \\ 532 & 1.8368 & 0.0930 \\ 808 & 1.8217 & 0.0948 \\ 945 & 1.8186 & 0.0943 \\ 1030 & 1.8173 & 0.0942 \\ 1064 & 1.8169 & 0.0941 \\ 1333 & 1.8146 & 0.0938 \\ 1444 & 1.8140 & 0.0937 \\ 1500 & 1.8137 & 0.0936 \\ 1540 & 1.8132 & 0.0936 \\ 2014 & 1.8123 & 0.0934 \\ 2097 & 1.8121 & 0.0934 \\ 2123 & 1.8121 & 0.0934 \\ 2940 & 1.8113 & 0.0933\end{array}$

\section{Nd:YAG Crystal Devlce Speciflcations}

Nd Concentrations Avalable 0.2 to $1.4 \mathrm{~K}$

Standard Rod Dimensions

- Diameter

$0.5 \mathrm{~mm}$ to $15.0 \mathrm{~mm}$

- Length

$10 \mathrm{~mm}$ to $220.0 \mathrm{~mm}$

- Slabs

Mnisture to large configurations

Laser Rod Specifications

Transmitted Wavetront Standard Grade Premium Grade

(per inch of rod lergth) $\quad$ N8 $\quad$ N/20

- Extinction Ratio Greater than 25 db

- Surface Quality 1015

- Clear Aperture 95\%

- Paralledism $\$ 10$ arc seconds

- Perpendicularity $\quad \$ 5$ minutes

- Surface Flatness 210 율 $632.8 \mathrm{~nm}$

- Barrel Finish Ground or Poilshod

- Chatfer

- Diameter Tolerance

$0.13+1-0.08 \mathrm{~mm} \oplus 45$ "

- Length Tolerance

$+0.0,-0.025 \mathrm{~mm}$

$+1.0 .5 \mathrm{~mm}$

Laser/Spectroscoplc

Configurations

- Flatiparales

- Tilt Endt

- Radus Ends

- Brewsterilarewster Ends

- Siab Desigre

- Polished and Grooved Barrels

- Diode Pumpod Mni-rods

- Custom Geometris

Thin Film Coatings

- ARJAR at $1064 \mathrm{~nm}, \mathrm{R}<0.15 \%$ Damage Threshold $>20 \mathrm{Jkm}^{2}, 10 \mathrm{rE}$ pulse

\begin{tabular}{|c|c|}
\hline $4 F_{y 2}$ to $\left.4\right|_{112}$ & Stark level transitions \\
\hline Transition wavelongth (hm) & Normalzed output intensity \\
\hline 1052.1 & 0.300 \\
\hline 1054.9 & 0.018 \\
\hline 1051.5 & 0.627 \\
\hline 1054.1 & 1.000 \\
\hline 1064.6 & 0.418 \\
\hline 1068.2 & 0.267 \\
\hline 1073.7 & 0.515 \\
\hline 1077.9 & 0.363 \\
\hline 1105.5 & 0.114 \\
\hline 1111.9 & 0.233 \\
\hline 1115.8 & 0.279 \\
\hline $\begin{array}{c}{ }^{4} F_{y z} \text { bo } 4 / 13 z \\
13187\end{array}$ & $\begin{array}{l}\text { Stark level transitions } \\
0.144\end{array}$ \\
\hline 1320.3 & 0.048 \\
\hline 1333.5 & 0.057 \\
\hline 1335.1 & 0.078 \\
\hline 1338.1 & 0.191 \\
\hline 1341.9 & 0.094 \\
\hline 1353.3 & 0.049 \\
\hline 1357.2 & 0.168 \\
\hline 1415.0 & 0.078 \\
\hline 1427.1 & 0.022 \\
\hline 1432.0 & 0.052 \\
\hline 1444.4 & 0.100 \\
\hline
\end{tabular}

- Dichroics, HR $1064 \mathrm{~nm}>99.8 \% R$, HT $808 \mathrm{~nm}>95.0 K \mathrm{KT}$ Damage Threshold > $15 \mathrm{llcm}$, $10 \mathrm{rs}$ pulse

- Partialy Reflocting Desigre

- Custom Designs Availabie 
Material Properties Duss

Optical Proportios

Nafractire index

Primary Diode Purmp Bare

Alomsernes Ifstime

1.8169 at $1054 \mathrm{~mm}$

$306.5 \mathrm{~mm}$

$230 \mu \mathrm{s} 91.0 \mathrm{~s}$ Nd

Thormal Proportios

Thomal Conductivty $(20 \mathrm{C})$

Spoific Haat

0.129 Wham $k$

$0.59 \mathrm{~V}-\mathrm{K}$

$8.2 \times 10-4 K<100>$

$1.7 \times 10^{-6} \mathrm{~K}<110$ :

$7.8 \times 10^{-6} \mathrm{~K}<111$ ?

$3 \times 10^{10}$

$175-200$ Wom

$+8.9 \times 10^{6} \mathrm{~K}^{-1}$

Descipative Fracturs Limit

andet

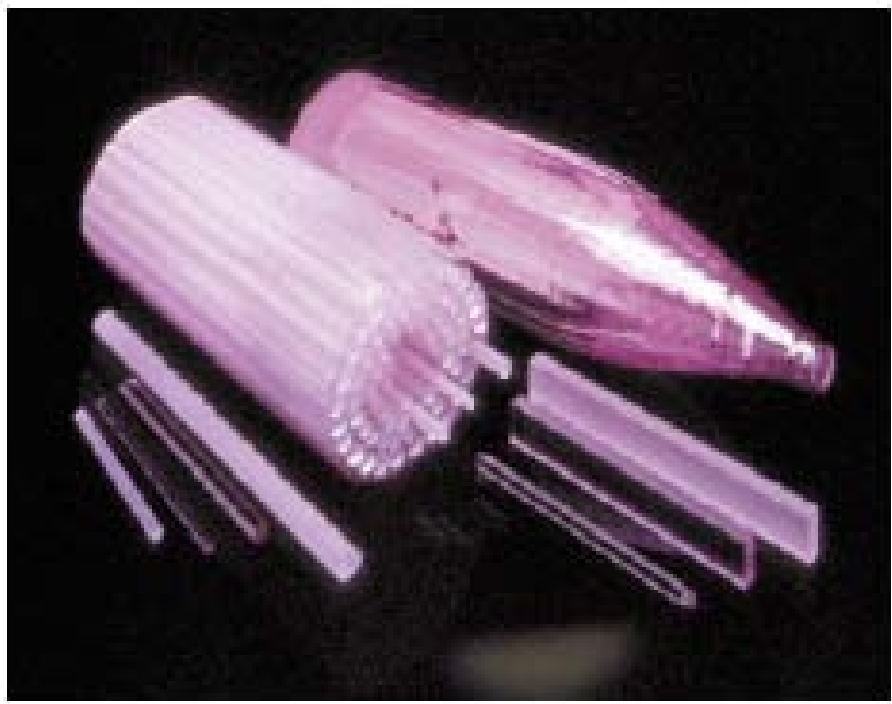

Physical Properties

Nd Dophng Lesel

Chamical formit:

Malocitr Moght

Crystal Sructurt

Latsece Coretant

Matire Point

Dereity

Knoop Hartings

0.2 to 1.48 atomi:

$\mathrm{Y}_{3} \mathrm{Nad}_{3} \mathrm{Al}_{5} \mathrm{O}_{12}$

505.3 g/moks

Cutic I gernet

$1201 \mathrm{~A}$

$1920 \mathrm{C}$

$4.55 \mathrm{gmiam}^{3}$

$1350+1-35 \mathrm{kgmm}^{2}$

Mochanical Proporties Youngs Moduls

Tarele Strangh

$282 \mathrm{CPa}\left(4 \mathrm{sin} 10^{6} \mathrm{psi}\right)$

$2 \mathrm{BD} \mathrm{MPz}$

0.210

\section{REFERENCES}

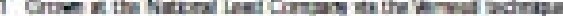

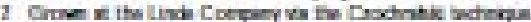

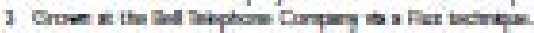

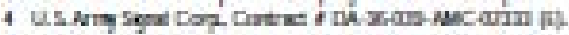

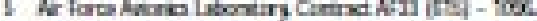

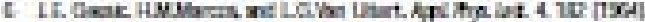

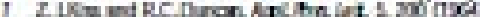

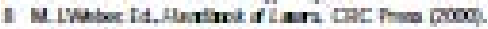

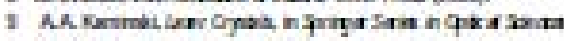

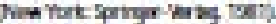

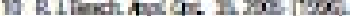

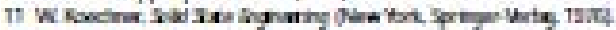

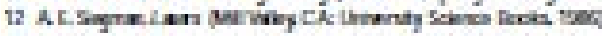

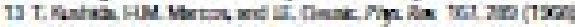

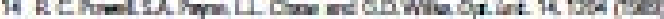

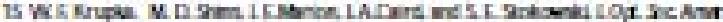

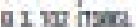

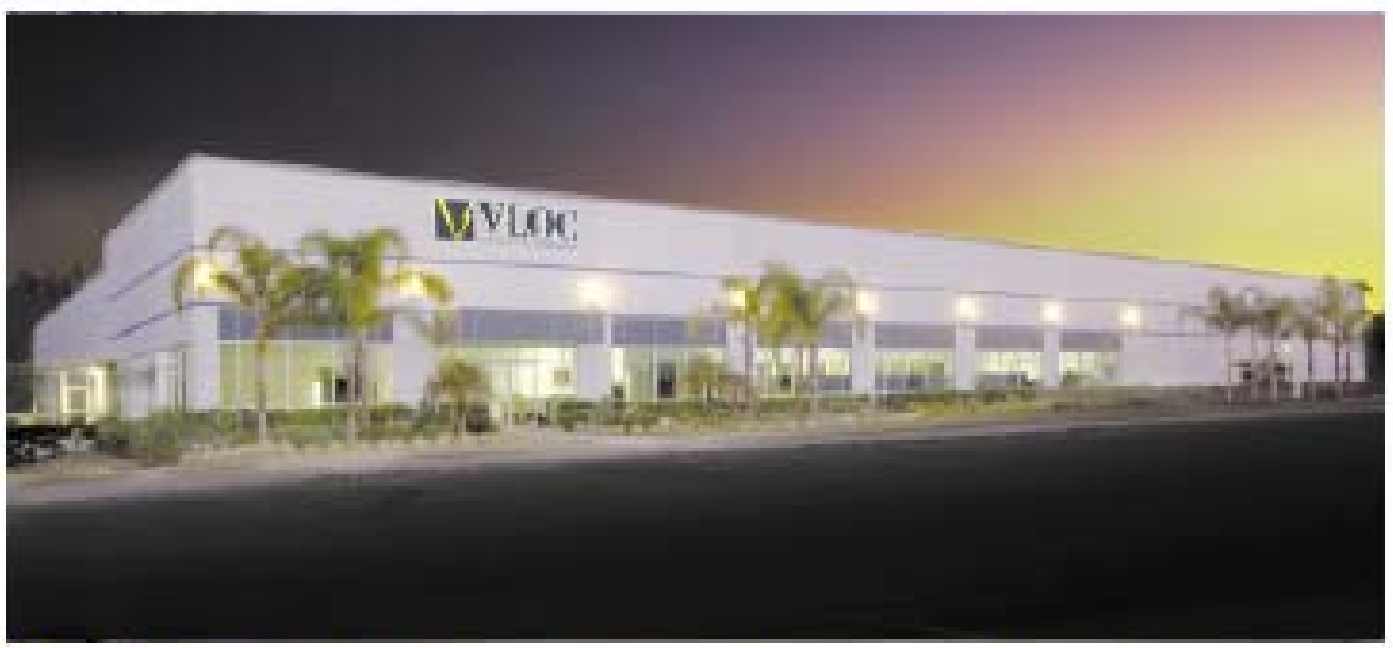

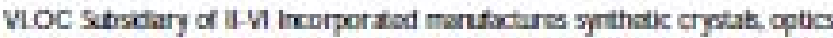

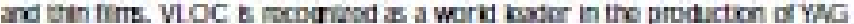
products far industrti, sciontife, motical, delonse and resarch applcrions.

7826 Phooonics Drive, New Port Richer, FL 34655 Phoos: 727-375-VLOC (8562) Fax: 727-375-5300 E-mail infoifvioc.cem Wabaite awarioc.com 


\section{Output Coupler and High Reflection Mirror}

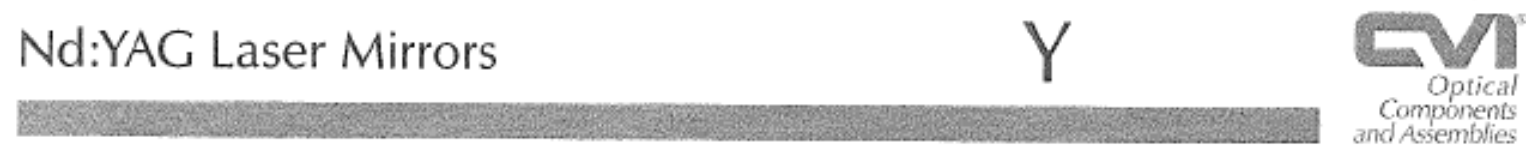

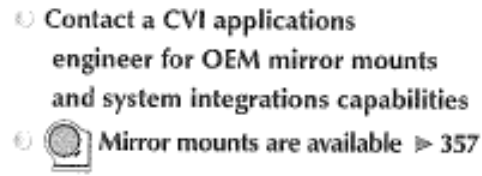

Substrate Material UV grade fused silica if $\lambda<450 \mathrm{~nm}$

$$
\text { BK7 glass if } \lambda \geq 450 \mathrm{~nm}
$$

S1 Surface Figure $N / 10$ at $633 \mathrm{~nm}$ before coating

\$1 Surface Quality 10-5 CVI Laser Quality defined on page $\mathbf{4 3 0}$

\$2 Surface Quality Commercial polish

Diameter Tolerance $+0.00 \mathrm{~mm},-0.25 \mathrm{~mm}$

Thickness Tolerance $\pm 0.25 \mathrm{~mm}$

Wedge $\leq 5$ minutes

Chamfer $0.35 \mathrm{~mm}$ at $45^{\circ}$ typical

Concentricity $\leq 0,05 \mathrm{~mm}$

Radius Tolerance $\pm 0.5 \%$

Coating Technology Electron beam multilayer dielectric Adhesion and Durability Per.MIL-C-675C. Insoluble in lab solvents.

Clear Aperture Exceeds central $85 \%$ of diameter

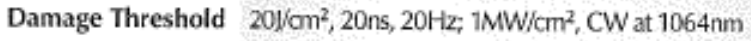

Coated Surface Figure $N / 10$ at $633 \mathrm{~nm}$ on select substrates

How To Order

\begin{tabular}{lcc} 
Product Code & Laser & Wavelength \\
\hline$Y_{1}$ & Nd:YAG & $1064 \mathrm{~nm}$ \\
$Y_{2}$ & Nd:YAG & $532 \mathrm{~nm}$ \\
$Y 3$ & Nd:YAG & $355 \mathrm{~nm}$ \\
$Y 4$ & Nd:YAC & $266 \mathrm{~nm}$ \\
$Y 5$ & Nd:YAG & $213 \mathrm{~nm}$ \\
$Y 13$ & Nd:YAG & $1319 \mathrm{~nm}$
\end{tabular}

\begin{tabular}{llllllllll} 
Size Code & Diameter & Thickness & & & \\
\hline 0537 & $0.500^{\prime \prime}$ & $0.375^{\prime \prime}$ & 1025 & $1.000^{\prime \prime}$ & $0.250^{A}$ & 1940 & $50.0 \mathrm{~mm}$ & $10.0 \mathrm{~mm}$ \\
0643 & $15.0 \mathrm{~mm}$ & $11.0 \mathrm{~mm}$ & 1032 & $1.000^{*}$ & $8.0 \mathrm{~mm}$ & 2037 & $2.000^{\prime}$ & $0.375^{\prime \prime}$ \\
0737 & $0.750^{\prime \prime}$ & $0.375^{\prime \prime}$ & 1037 & $1.000^{\prime \prime}$ & $0.375^{\prime \prime}$ & 3050 & $3.000^{\prime \prime}$ & $0.500^{\prime \prime}$ \\
$092+$ & $25.0 \mathrm{~mm}$ & $6.0 \mathrm{~mm}$ & 1537 & $1.500^{\prime \prime}$ & $0.375^{\prime}$ & 4050 & $4.000^{\prime \prime}$ & $0.500^{\prime \prime}$
\end{tabular}

Angle of Incidence in Degrees with Polarization

\begin{tabular}{ll}
$45 S$ & Normal incidence \\
$45 \mathrm{P}$ & 45 degrees, $S$ polarization \\
45 LINP & 45 degrees, $P$ polarization \\
\hline
\end{tabular}

Radius of Curvature $(\mathrm{m})(\mathrm{CC}=$ concave, $\mathrm{CX}=$ convex, omit for flat mirror) $D 196,428$

$\begin{array}{llllllll}0.025 & 0.10 & 0.25 & 0.75 & 1.5 & 4.0 & 7.0 & 10.0 \\ 0.05 & 0.15 & 0.30 & 1.0 & 2.0 & 5.0 & 8.0 & 15.0 \\ 0.075 & 0.20 & 0.50 & 1.2 & 3.0 & 6.0 & 9.0 & 20.0\end{array}$


$0^{\circ}$ designs
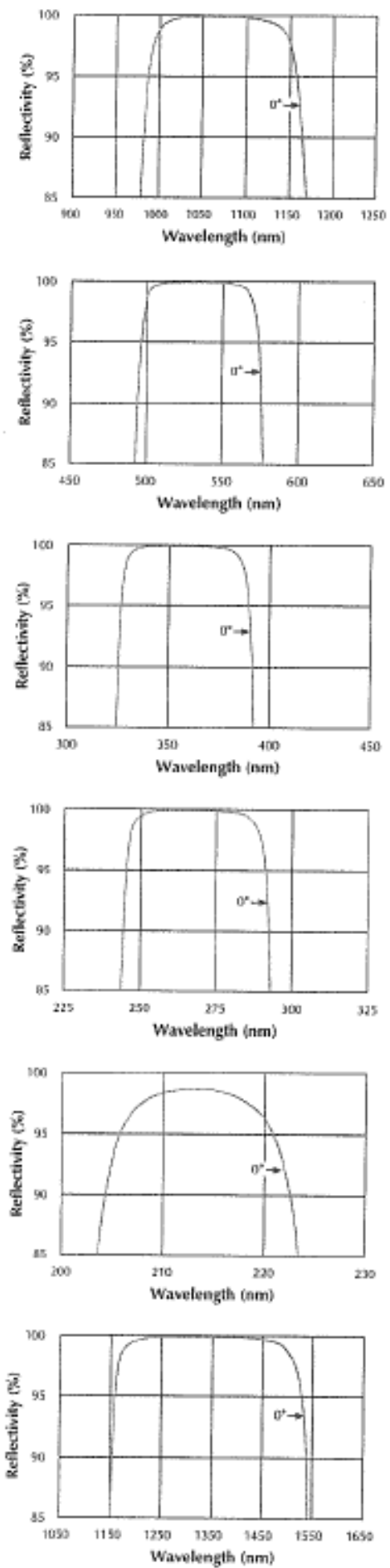

$45^{\circ}$ designs
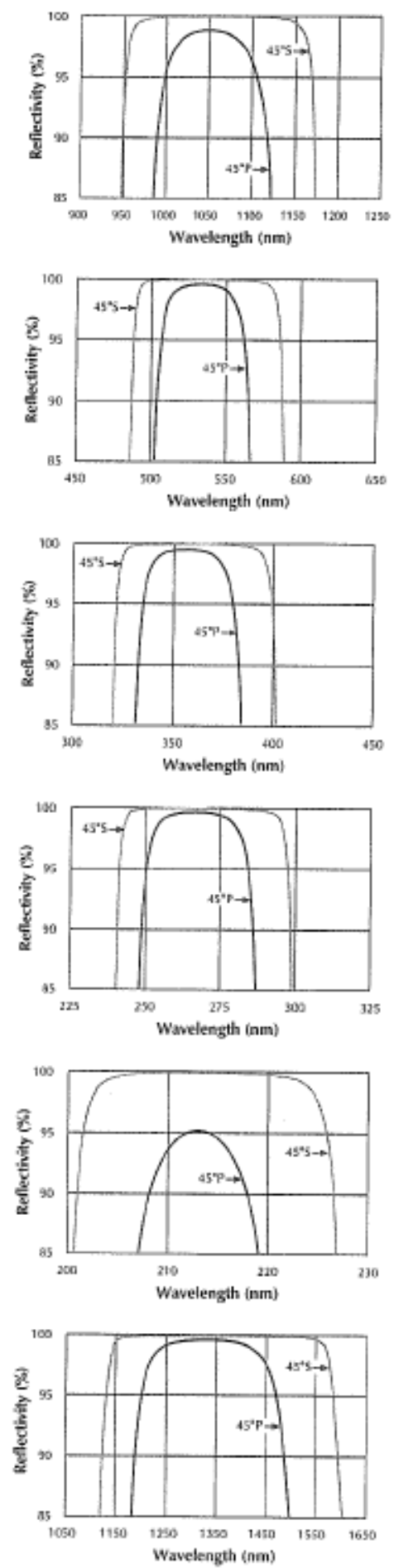

Reflectivity vs. Wavelength of Y7 Series $1064 \mathrm{~nm}$ Nd:YAC Laser Mirror for $0^{\circ}$ and $45^{\circ}$ incidence angle designs.

Min. Reflectance $>99.5 \%$ at $0^{\circ}$ $>99.0 \%$ at $45^{\circ}$ UNP

Reflectivity vs, Wavelength of YZ Series 532am Nd:YAC Laser Mirror for $0^{\circ}$ and $45^{\circ}$ incidence angle designs.

Min. Reflectance $>99.5 \%$ at $0^{\circ}$

$>99.0 \%$ at $45^{\circ} \mathrm{UNP}$

Reflectivity vs. Wavelength of $Y_{3}$ Series $355 \mathrm{~nm}$ Nd:YAC Laser Mirror for $0^{\circ}$ and $45^{\circ}$ incidence angle designs.

$$
\begin{aligned}
\text { Min. Reflectance } & >99.5 \% \text { at } 0^{\circ} \\
& >99.0 \% \text { at } 45^{\circ} \text { UNP }
\end{aligned}
$$

Reflectivity vs. Wavelength of Y4 Series $266 \mathrm{~nm}$ Nd:YAC Laser Mirror for $0^{\circ}$ and $45^{\circ}$ incidence angle designs.

$$
\begin{aligned}
\text { Min. Reflectance } & >99.5 \% \text { at } 0^{\circ} \\
& >99.0 \% \text { at } 45^{\circ} \text { UNP }
\end{aligned}
$$

Reflectivity vs. Wavelength of $Y 5$ Series $213 \mathrm{~nm} N \mathrm{Nd}$ :YAC Laser Mirror for $0^{\circ}$ and $45^{\circ}$ incidence angle designs.

$$
\begin{aligned}
\text { Min. Reflectance } & >98.0 \% \text { at } 0^{\circ} \\
& >97.0 \% \text { at } 45^{\circ} \text { UNP }
\end{aligned}
$$

Reflectivity vs. Wavelength of Y13 Series $1379 \mathrm{~nm}$ CW Nd:YAC Laser Mirror for $0^{*}$ and $45^{\circ}$ incidence angle designs.

Min. Reflectance $>99.5 \%$ at $0^{\circ}$

$>99.0 \%$ at $45^{\circ} \mathrm{UNP}$ 


\section{New Wave Research Tempest-20 Nd:YAG laser}

Tempest The following table shows the Tempest specifications for the different models Specifications that are available. The specifications are subject to change without notice.

\begin{tabular}{|c|c|c|c|c|}
\hline Energy ${ }^{1}$ & $1064 \mathrm{~nm}$ & $532 \mathrm{~nm}$ & $355 \mathrm{~nm}$ & $266 \mathrm{~nm}$ \\
\hline Tempest-10 Hz & $200 \mathrm{~mJ}$ & $100 \mathrm{~mJ}$ & $50 \mathrm{~mJ}$ & $30 \mathrm{~mJ}$ \\
\hline Tempest-20 Hz & $200 \mathrm{~mJ}$ & $100 \mathrm{~mJ}$ & $50 \mathrm{~mJ}$ & $30 \mathrm{~mJ}$ \\
\hline Tempest-30 Hz & $180 \mathrm{~mJ}$ & $90 \mathrm{~mJ}$ & $40 \mathrm{~mJ}$ & $20 \mathrm{~mJ}$ \\
\hline Tempest-300-10 Hz & $300 \mathrm{~mJ}$ & $180 \mathrm{~mJ}$ & $75 \mathrm{~mJ}$ & $45 \mathrm{~mJ}$ \\
\hline Tempest-300-20 Hz & $200 \mathrm{~mJ}$ & $130 \mathrm{~mJ}$ & $\mathrm{NA}$ & $\mathrm{NA}$ \\
\hline Energy stability ${ }^{2}$ & $\pm 2 \%$ & $\pm 3.5 \%$ & $\pm 6 \%$ & $\pm 7 \%$ \\
\hline Pulse width ${ }^{3}$ & $3-5 n s$ & $3-5 n s$ & $3-5 n s$ & $3-5 n s$ \\
\hline Beam divergence ${ }^{4}$ & $<1 \mathrm{mrad}$ & $<1 \mathrm{mrad}$ & $<1 \mathrm{mrad}$ & $<1 \mathrm{mrad}$ \\
\hline Beam pointing 5 & $<200 \mu \mathrm{rad}$ & $<200 \mu \mathrm{rad}$ & $<200 \mu \mathrm{rad}$ & $<200 \mu \mathrm{rad}$ \\
\hline Jitter & $\pm 0.5 \mathrm{~ns}$ & $\pm 0.5 \mathrm{~ns}$ & $\pm 0.5 \mathrm{~ns}$ & $\pm 0.5 \mathrm{~ns}$ \\
\hline $\begin{array}{l}\text { Beam Diameter - } \\
\text { Tempest 10, 20, } 30\end{array}$ & $\sim 5 \mathrm{~mm}$ & $\sim 4.5 \mathrm{~mm}$ & $\sim 45 \mathrm{~mm}$ & $\sim 45 \mathrm{~mm}$ \\
\hline $\begin{array}{l}\text { Beam Diameter - } \\
\text { Tempest } 300-10,20\end{array}$ & $\sim 6 \mathrm{~mm}$ & $-55 \mathrm{~mm}$ & $-5.5 \mathrm{~mm}$ & $\sim 55 \mathrm{~mm}$ \\
\hline
\end{tabular}

1. Optical losses due to optional attenuator will reduce maximum energy by $10 \%$

4 Full angle for $86 \%$ of the energy, $1 / \mathrm{e}^{2}$ point

2 Pulse-to-pulse for $98 \%$ of shots after 30 minute warm up

5 Full angle for $86 \%$ of the energy, $1 / e^{2}$ point

3. Full width half maximum 


\section{Appendix-C: Screen Shots}

\section{Temperature: Front}

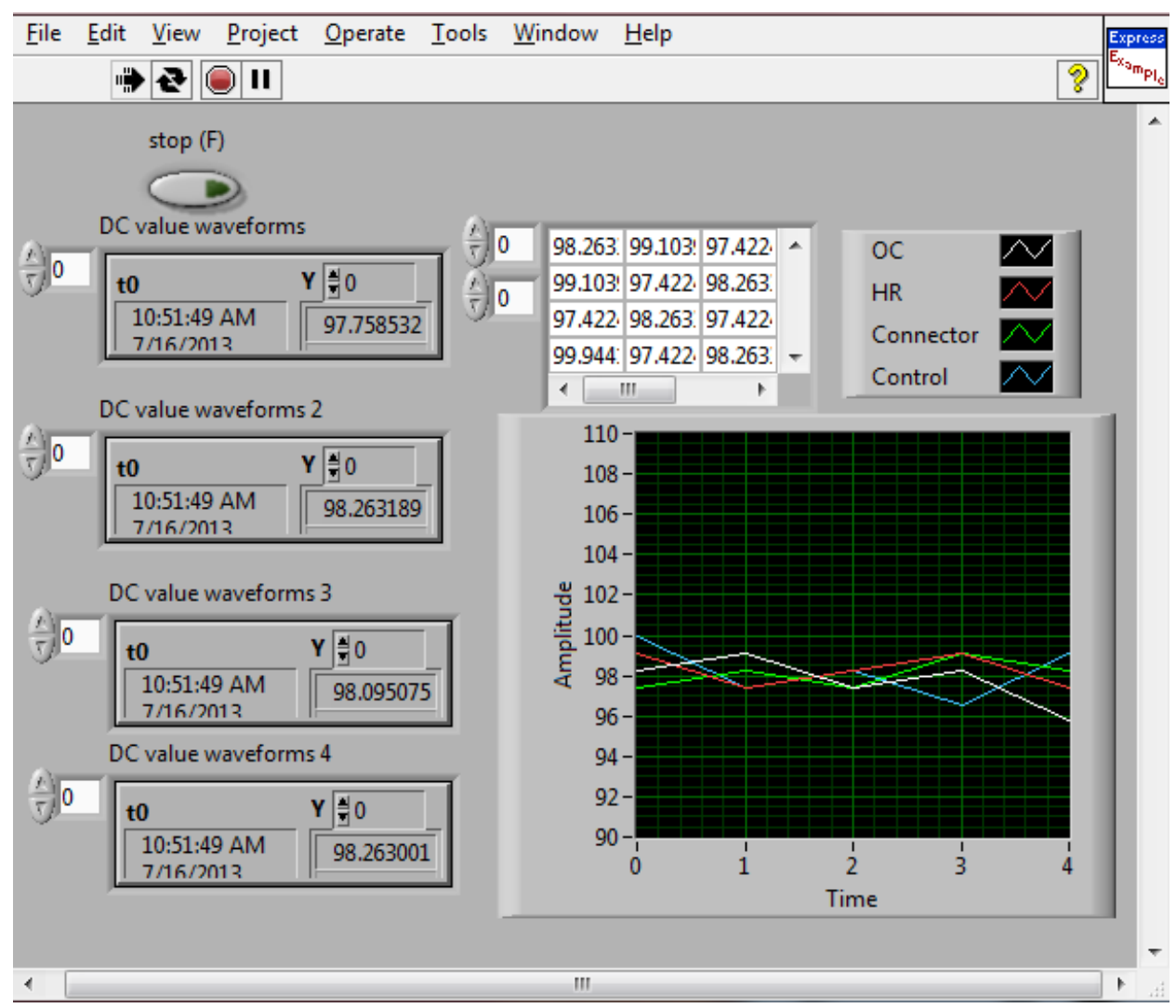

\section{Temperature: Back}

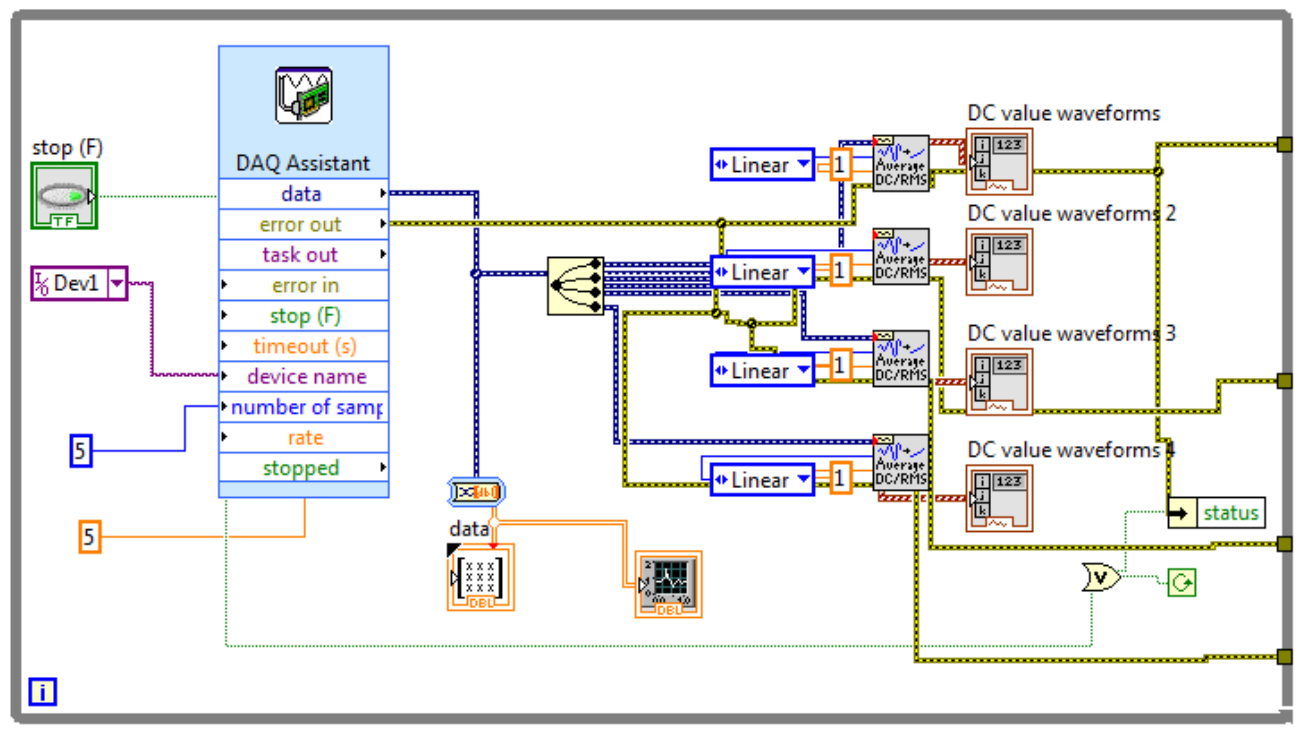




\section{Vibration: Front}

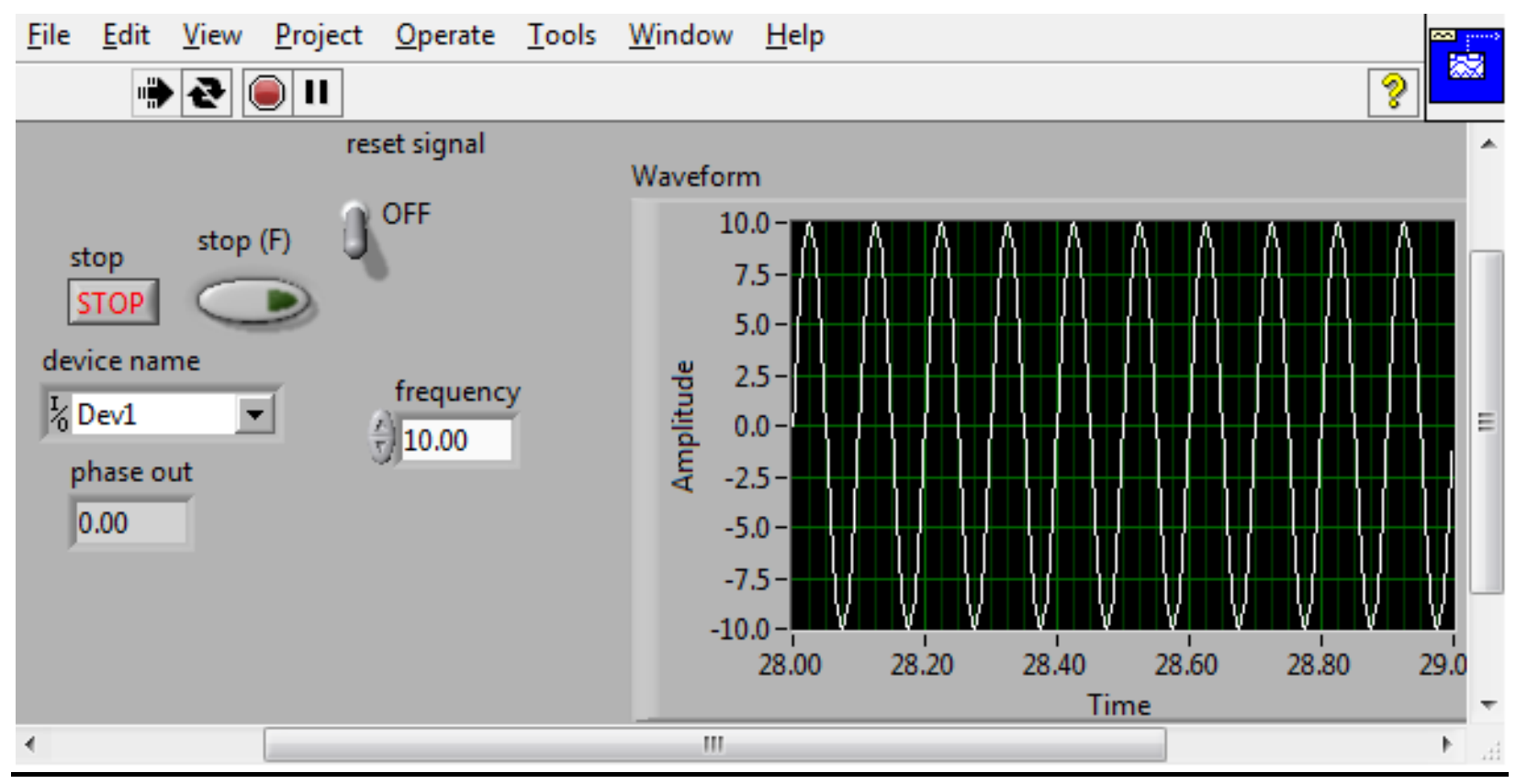

\section{Vibration: Back}

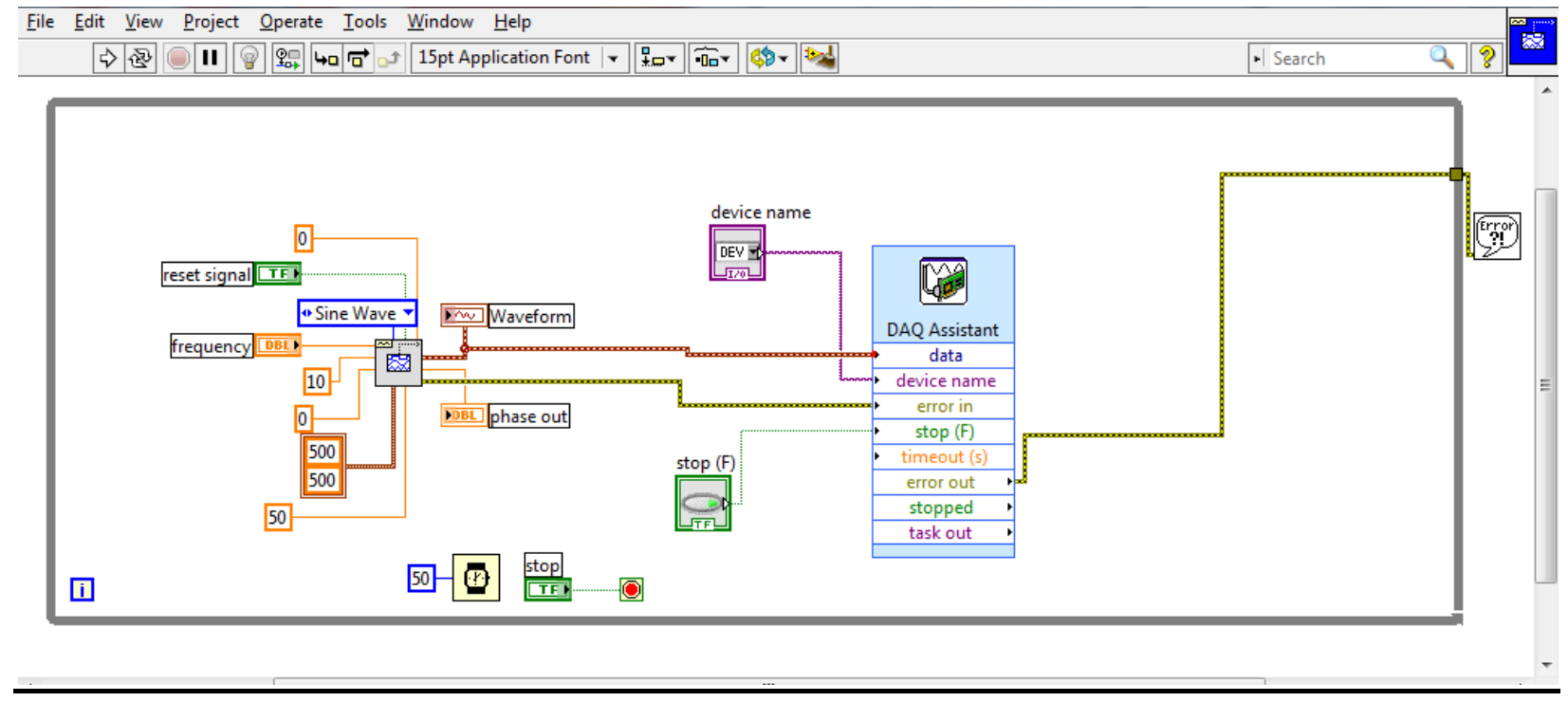




\section{CHR Interface}

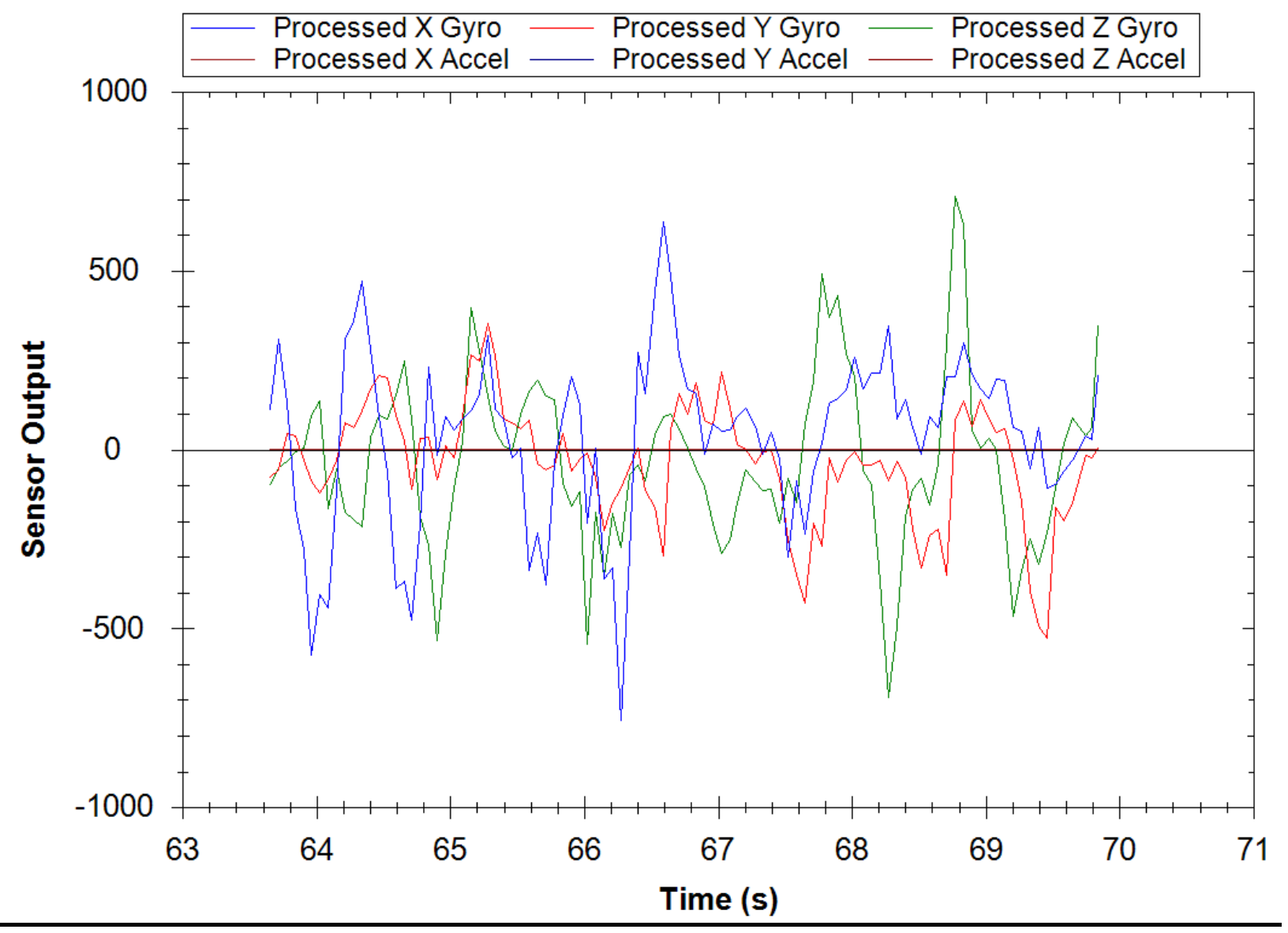




\section{Appendix-D: Technical Drawings}

\section{Laser Mount Adapter}

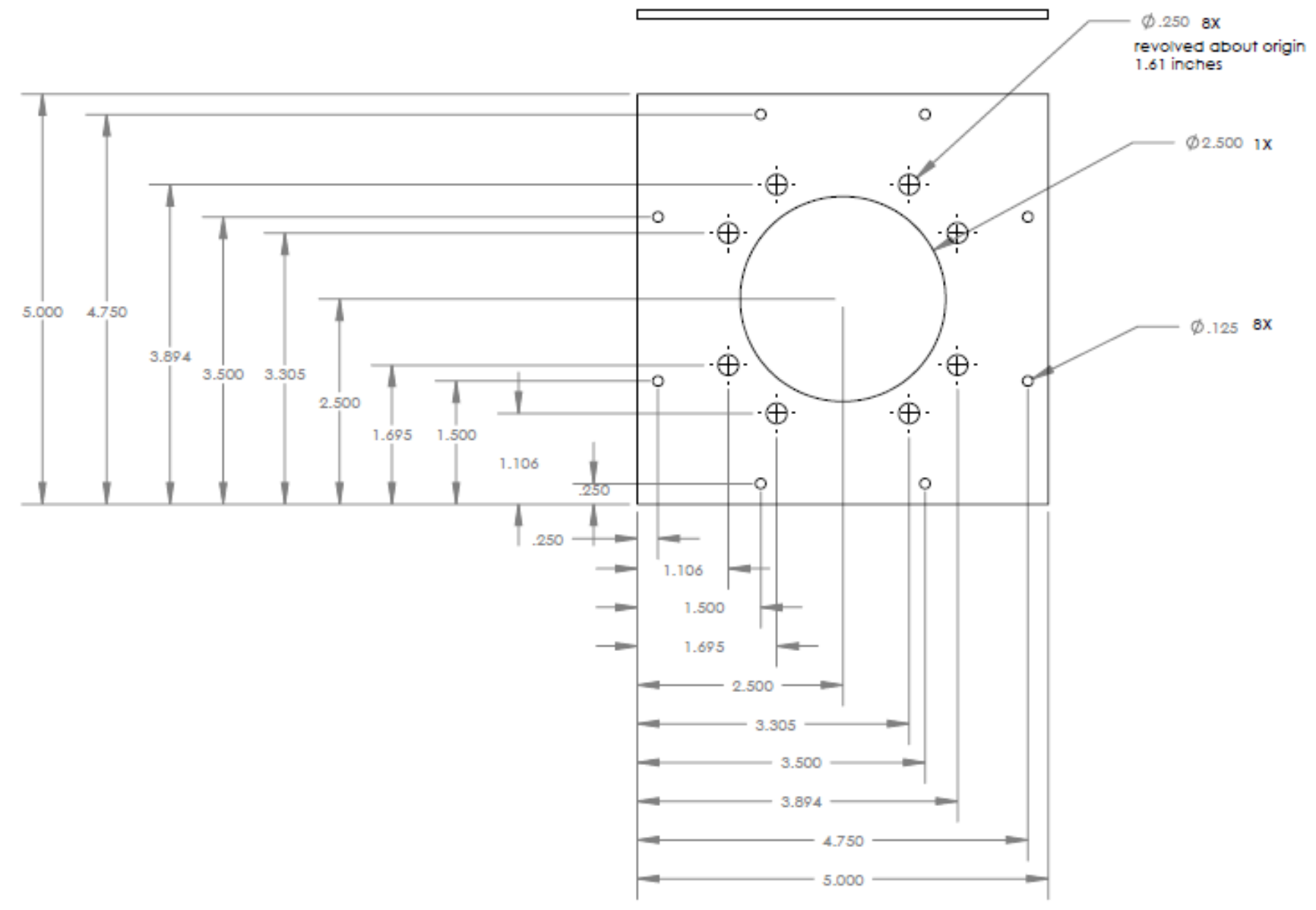


Vibrator Adapter Mount

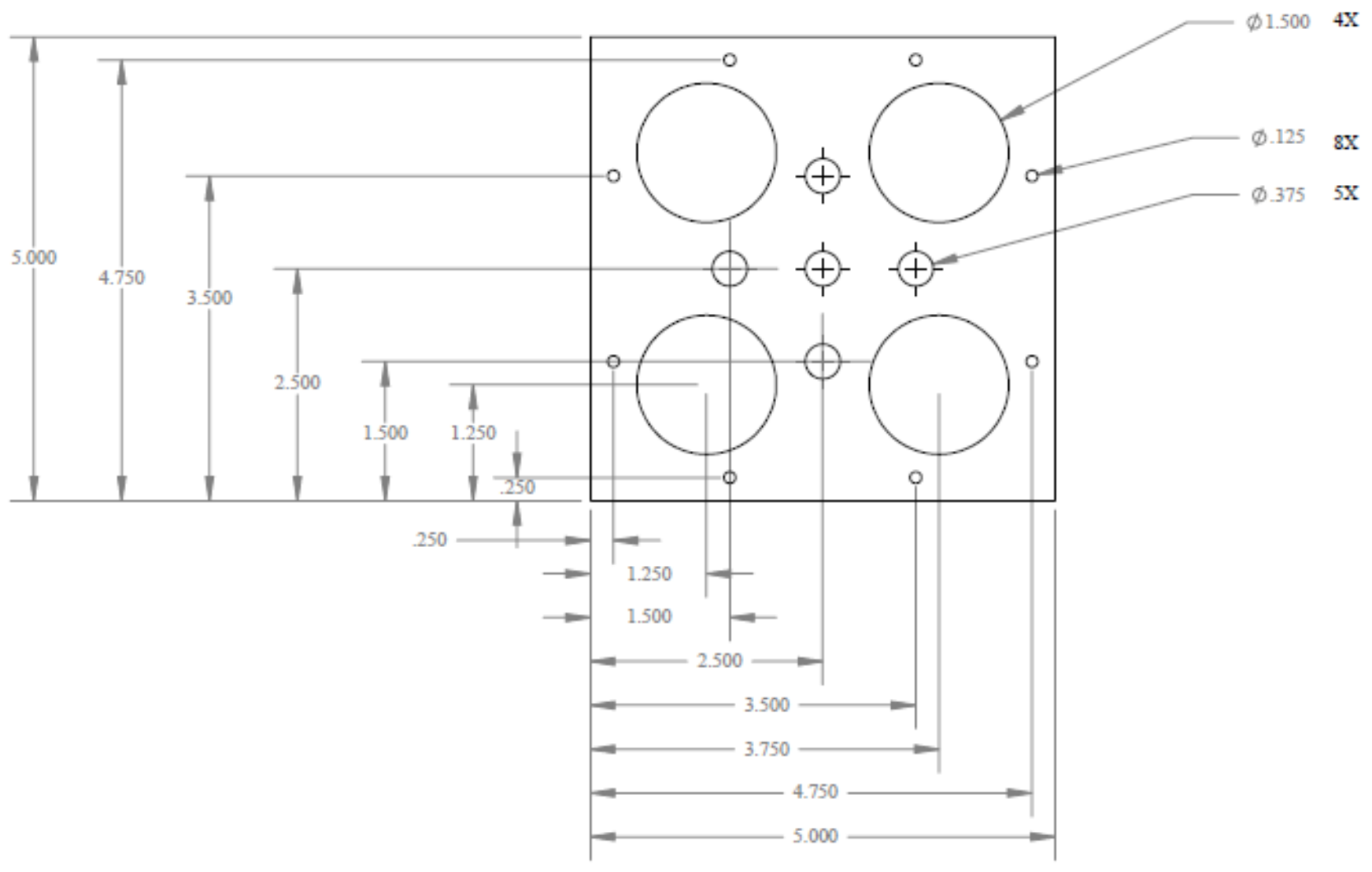




\section{Mirror Mount Mechanical Drawing}

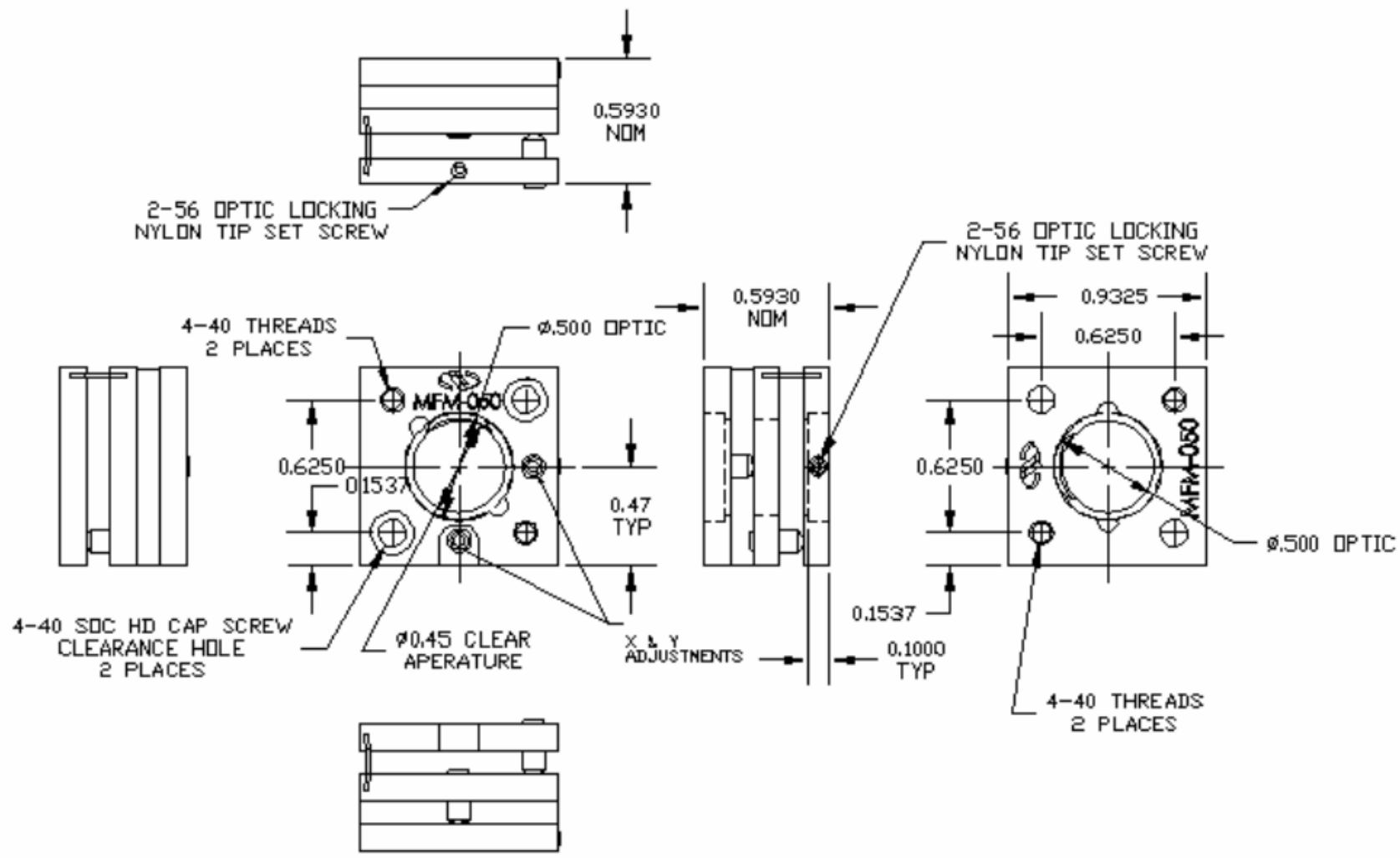




\section{Appendix-E: Additional Graphs}

\section{Q-switch Delay: 50\%}

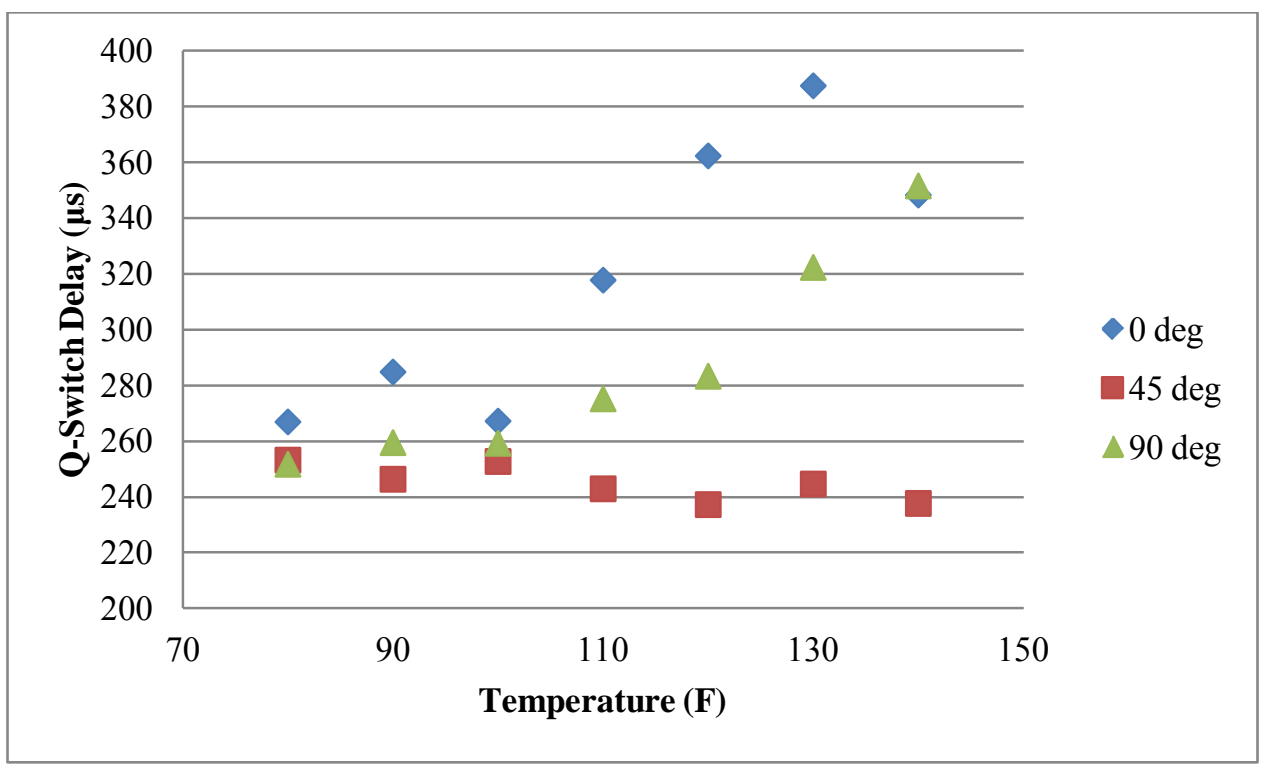

Figure 75: Q-switch delay as a function of temperature for the 0,45 and 90 degree mounting orientation with $50 \%$ acceleration

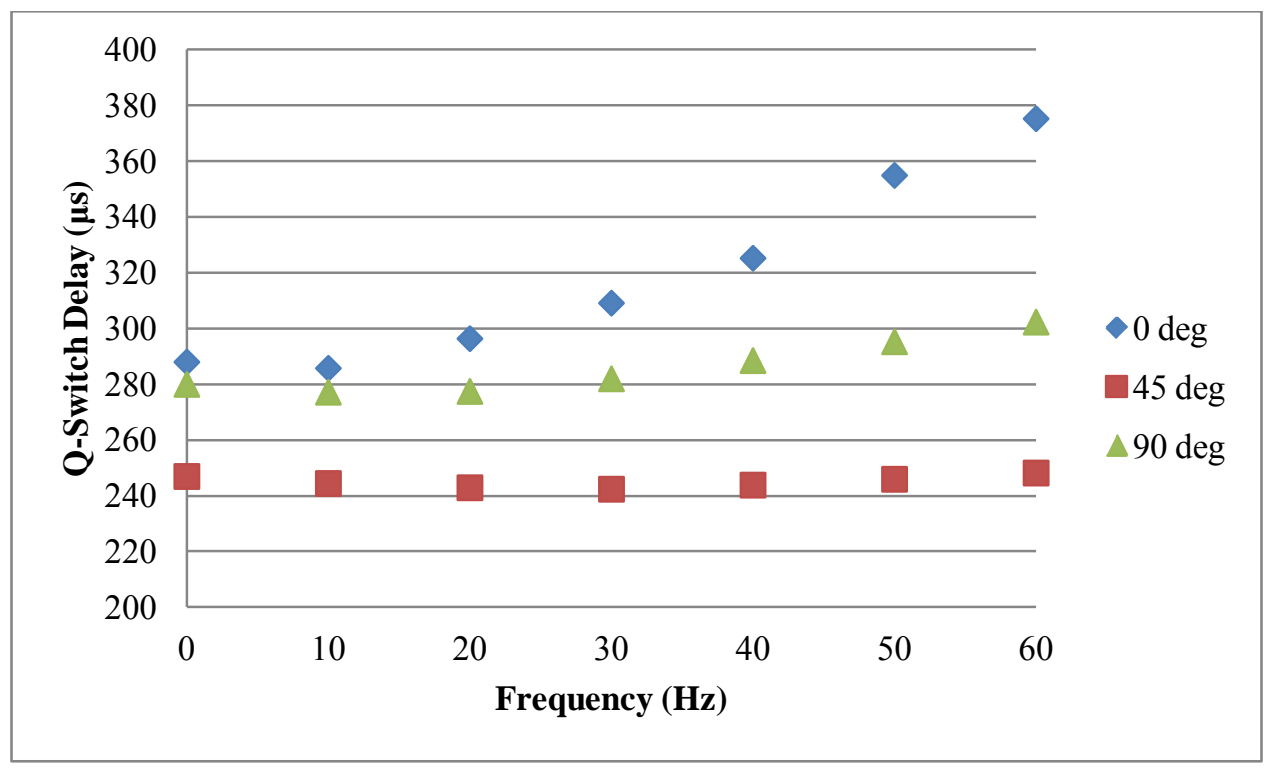

Figure 76: Q-switch delay as a function of frequency for the 0,45 and 90 degree mounting orientation with $50 \%$ acceleration 


\section{Pulse Width Variation: $50 \%$}

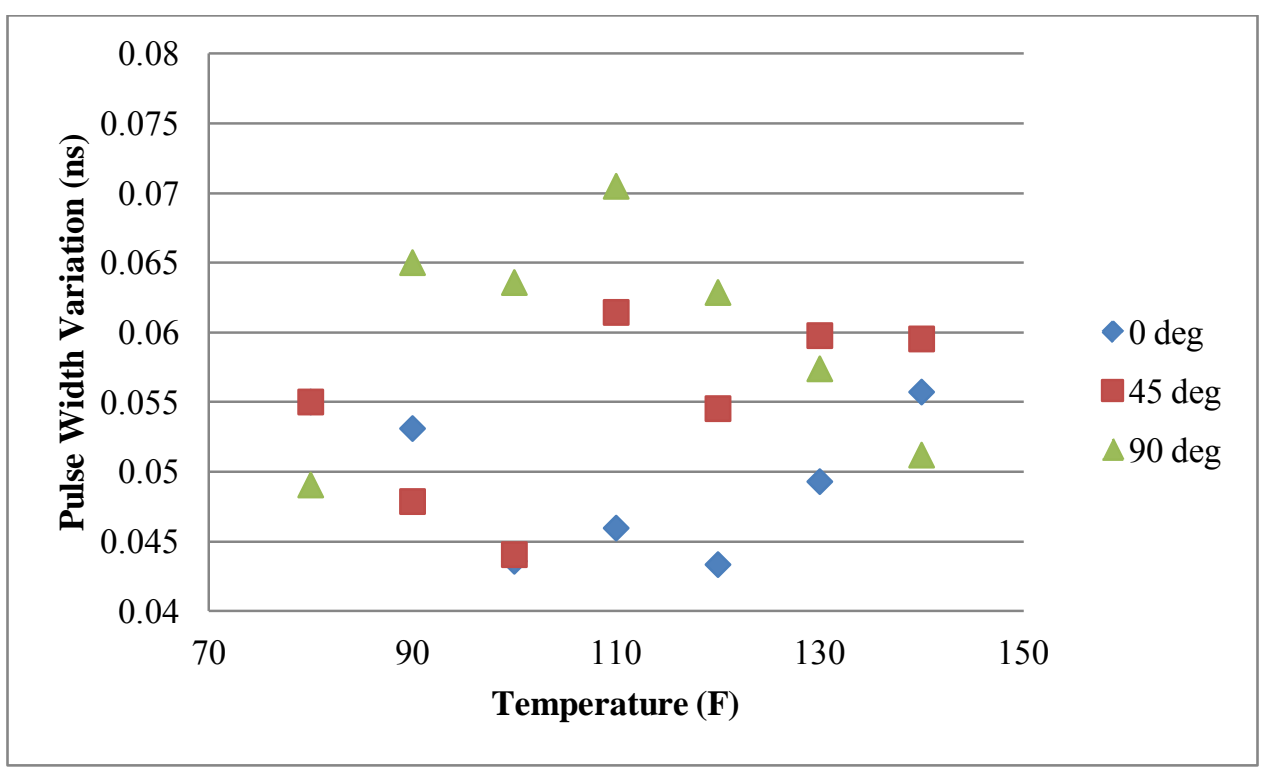

Figure 77: Pulse width variation as a function of temperature for the 0,45 and 90 degree mounting orientation with $50 \%$ acceleration

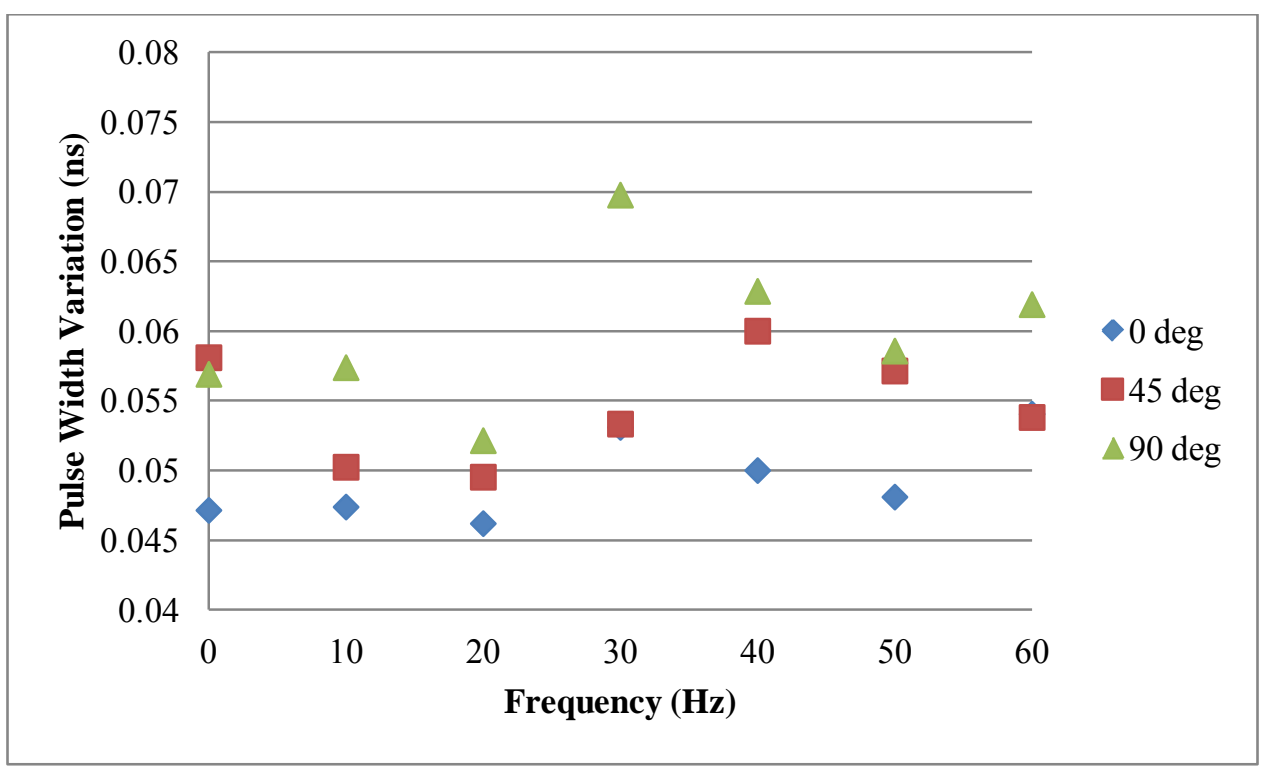

Figure 78: Pulse width variation as a function of frequency for the 0,45 and 90 degree mounting orientation with $50 \%$ acceleration 


\section{Pulse Width: $50 \%$}

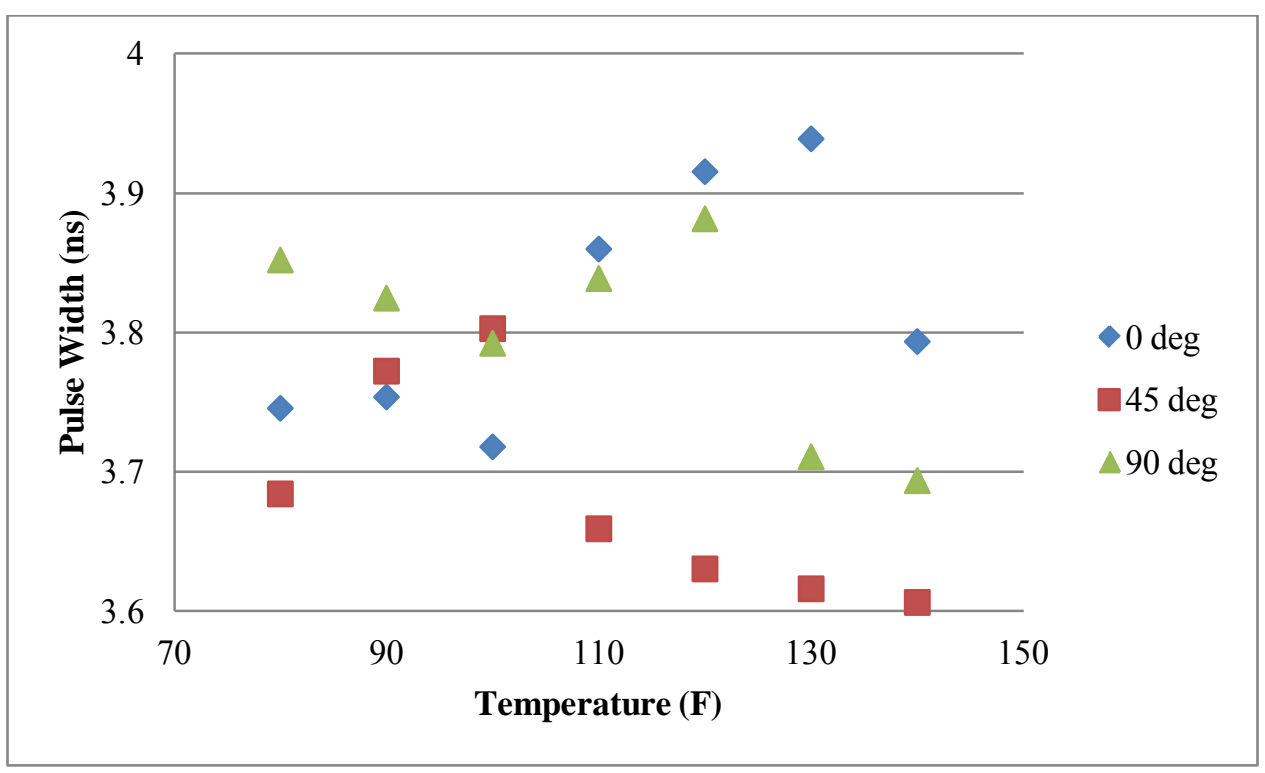

Figure 79: Pulse width as a function of temperature for the 0, 45 and 90 degree mounting orientation with $50 \%$ acceleration

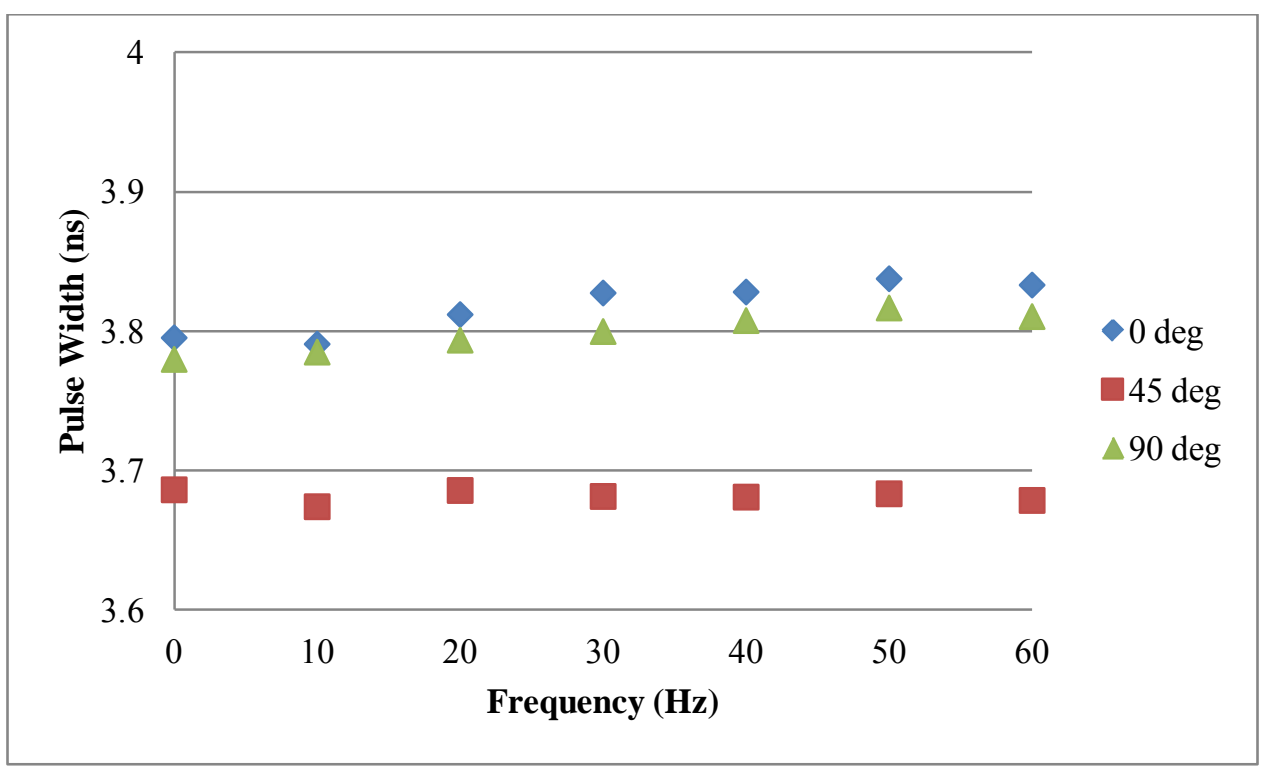

Figure 80: Pulse width as a function of frequency for the 0, 45 and 90 degree mounting orientation with $50 \%$ acceleration 


\section{Jitter: $\mathbf{5 0 \%}$}

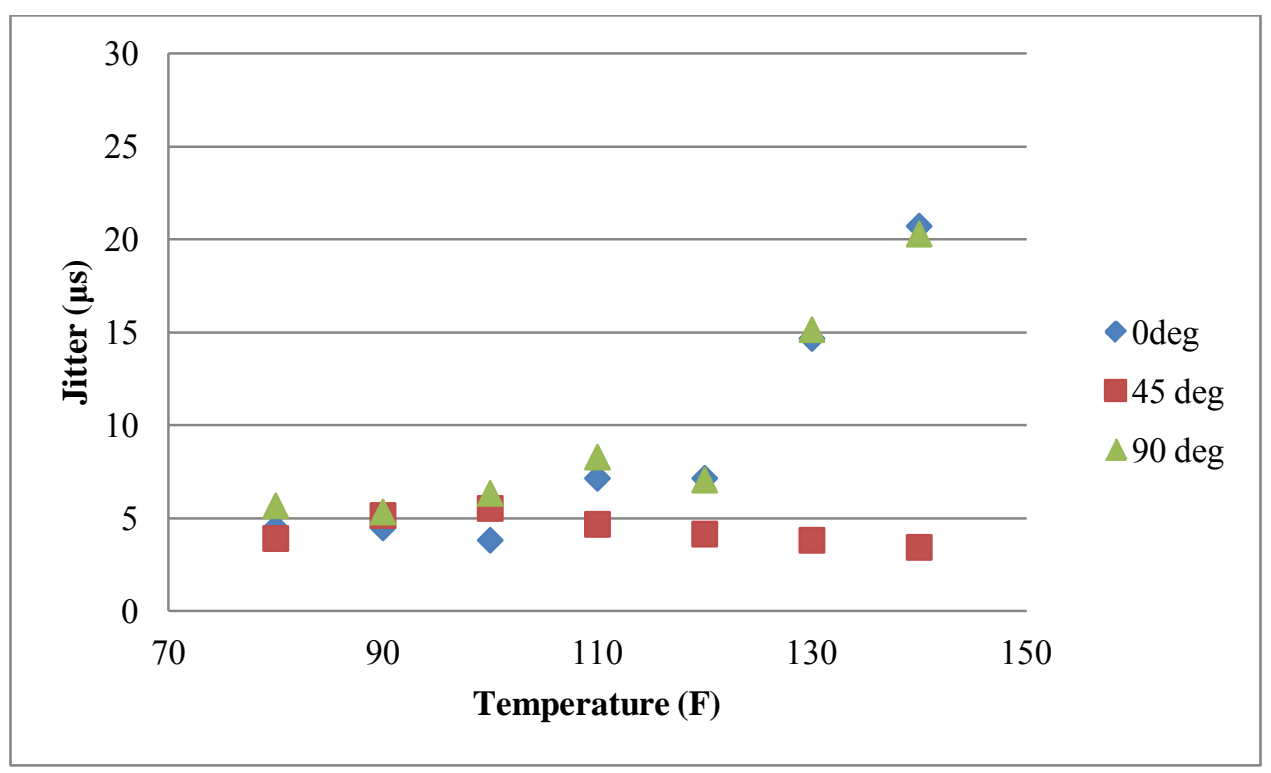

Figure 81: Jitter as a function of temperature for the 0,45 and 90 degree mounting orientation with $50 \%$ acceleration

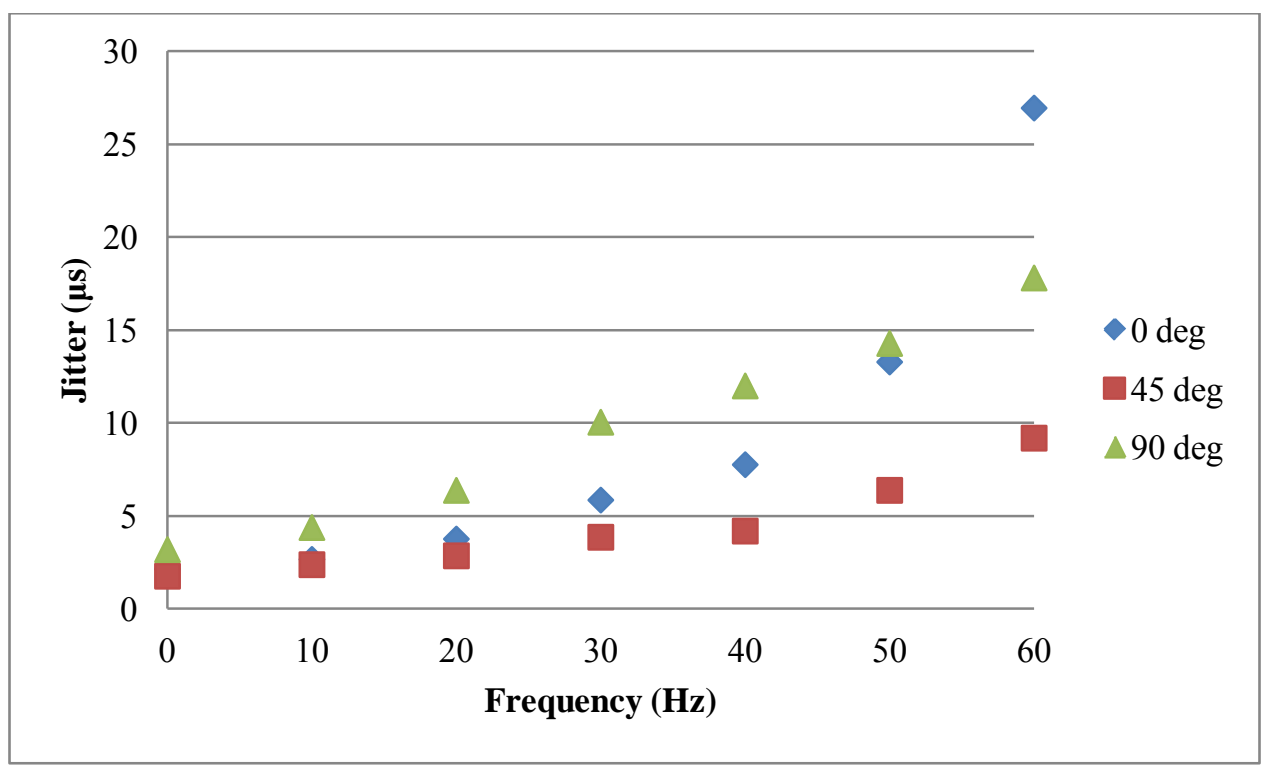

Figure 82: Jitter as a function of frequency for the 0,45 and 90 degree mounting orientation with $50 \%$ acceleration 


\section{Energy: 50\%}

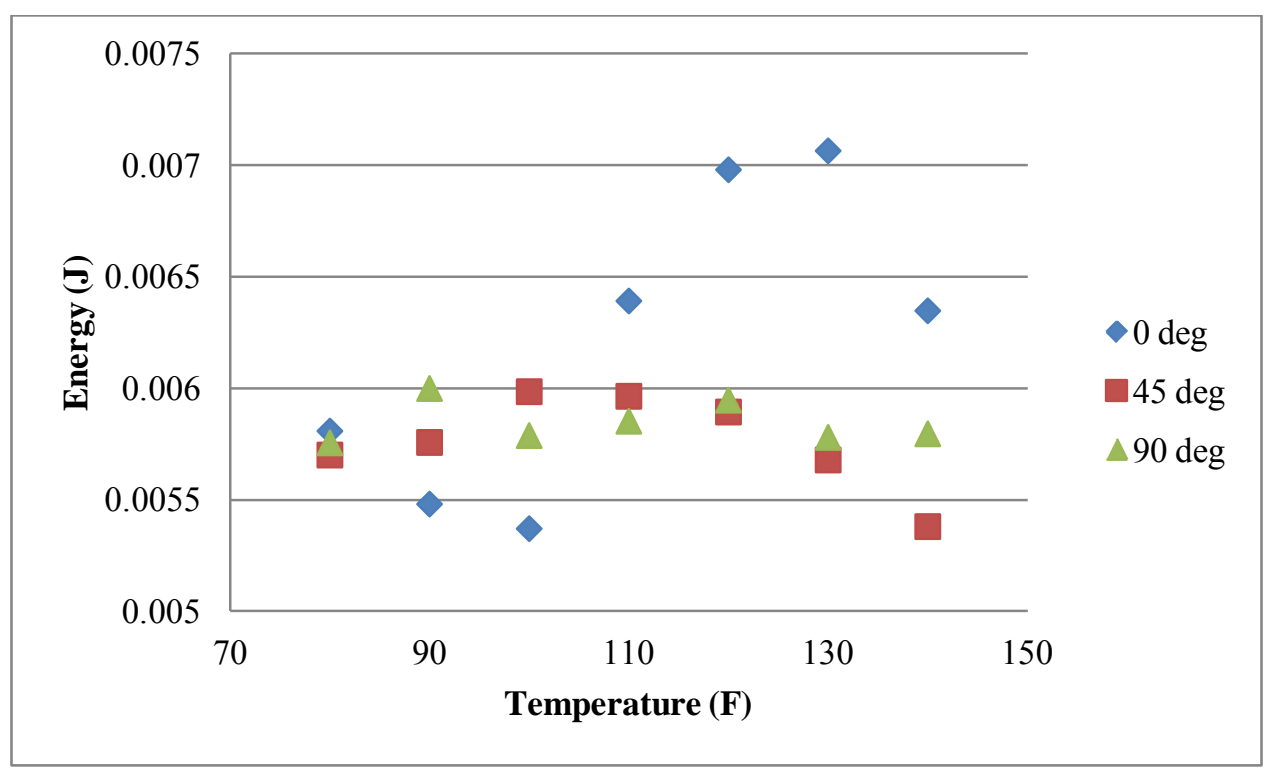

Figure 83: Energy as a function of temperature for the 0,45 and 90 degree mounting orientation with $50 \%$ acceleration

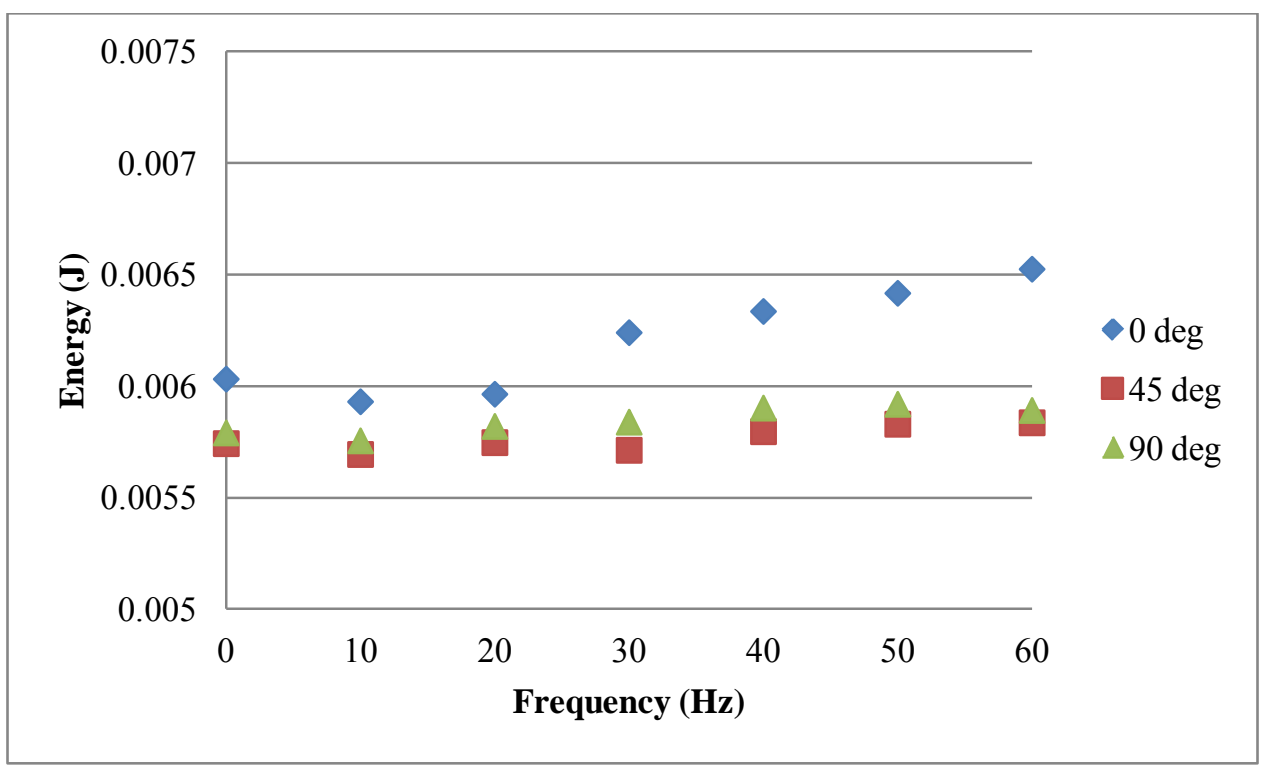

Figure 84: Energy as a function of frequency for the 0,45 and 90 degree mounting orientation with $50 \%$ acceleration 
Focal Intensity: $50 \%$

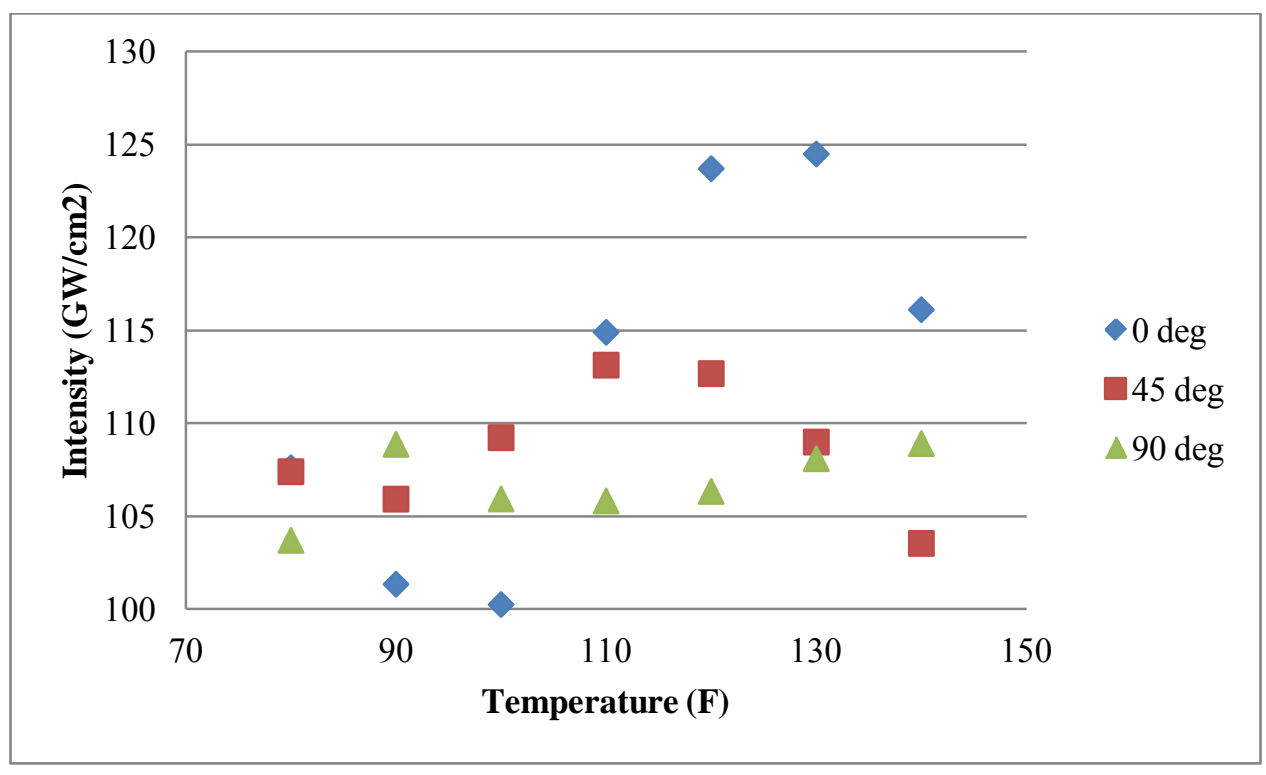

Figure 85: Intensity as a function of temperature for the 0,45 and 90 degree mounting orientation with $50 \%$ acceleration

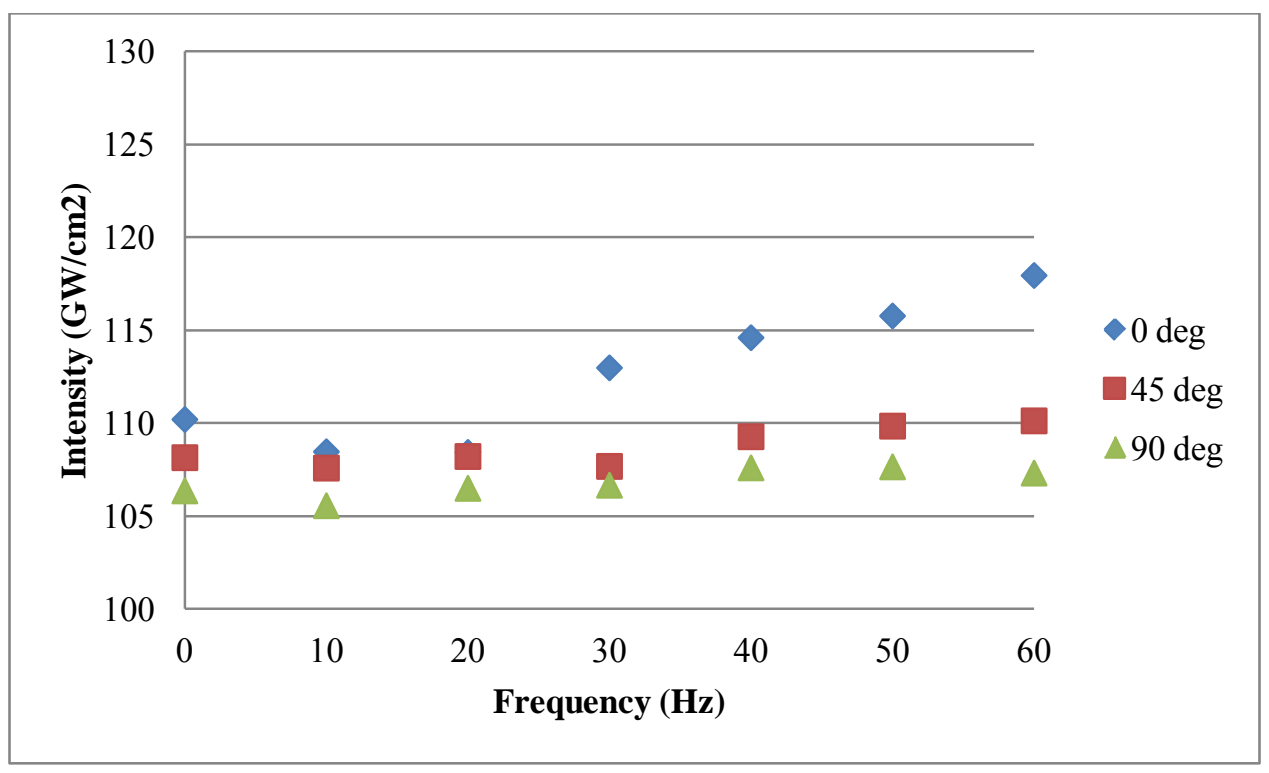

Figure 86: Intensity as a function of temperature for the 0,45 and 90 degree mounting orientation with $50 \%$ acceleration 


\section{Q-switch Delay: 100\%}

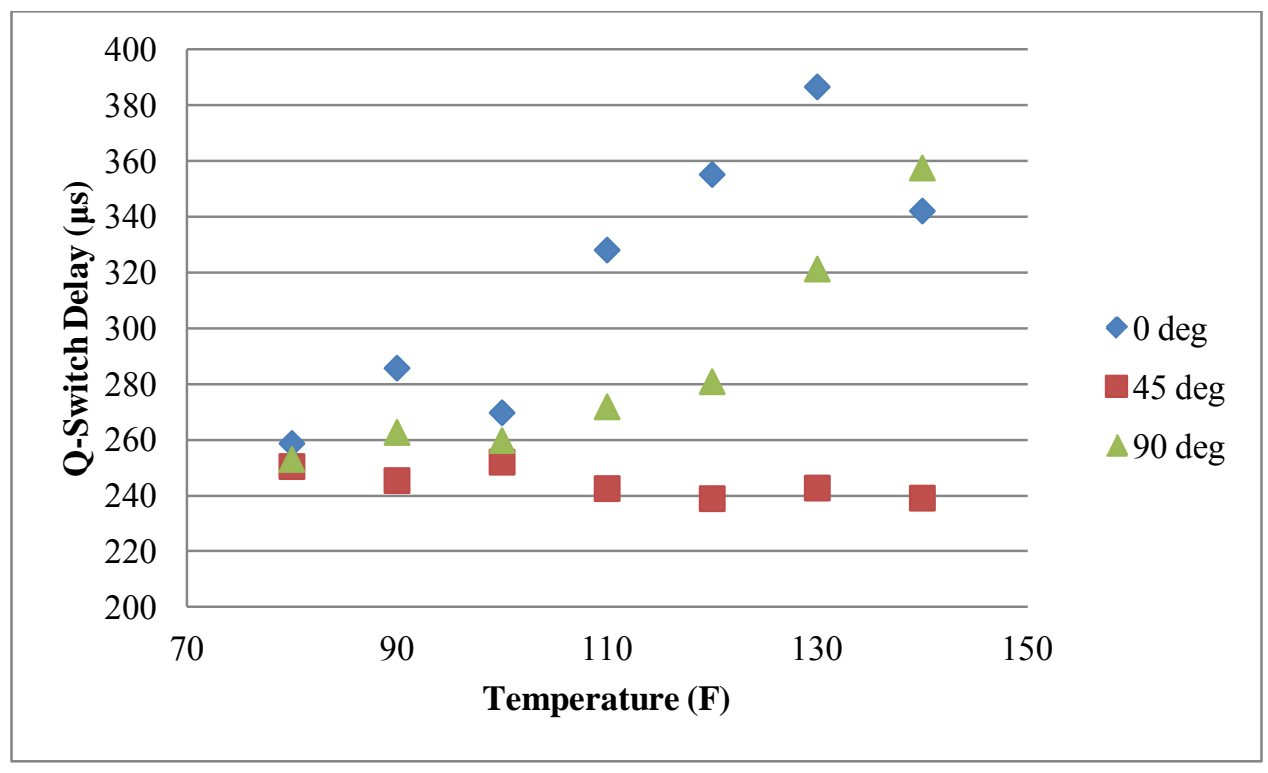

Figure 87: Q-switch delay as a function of temperature for the 0, 45 and 90 degree mounting orientation with $100 \%$ acceleration

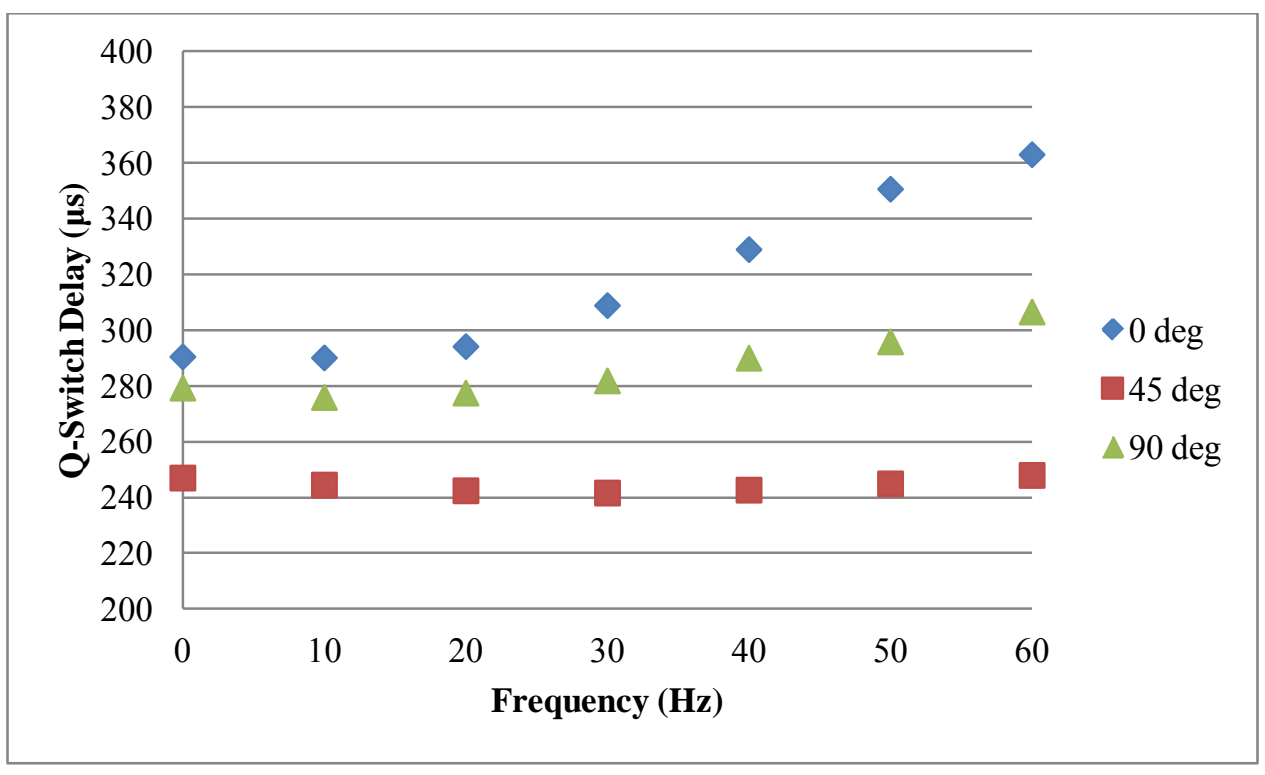

Figure 88: Q-switch delay as a function of frequency for the 0,45 and 90 degree mounting orientation with $100 \%$ acceleration 


\section{Pulse Width Variation: 100\%}

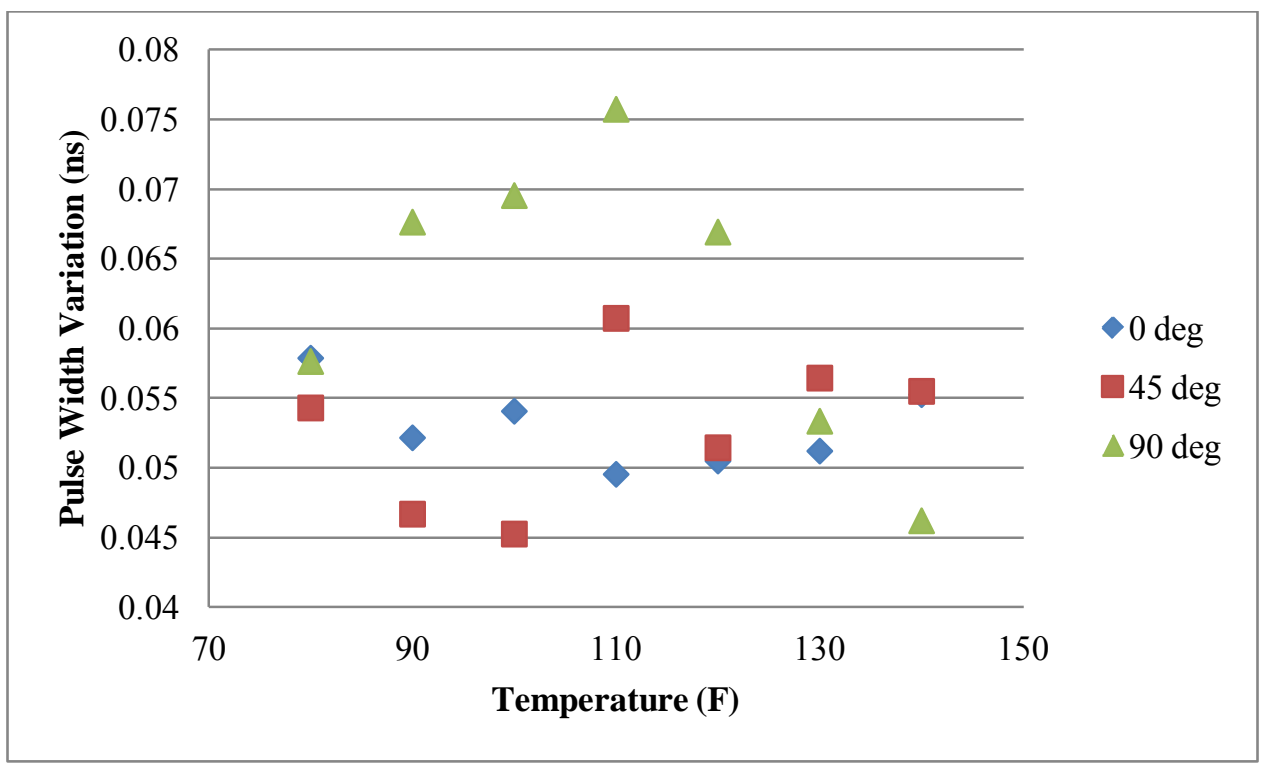

Figure 89: Pulse width variation as a function of temperature for the 0,45 and 90 degree mounting orientation with $100 \%$ acceleration

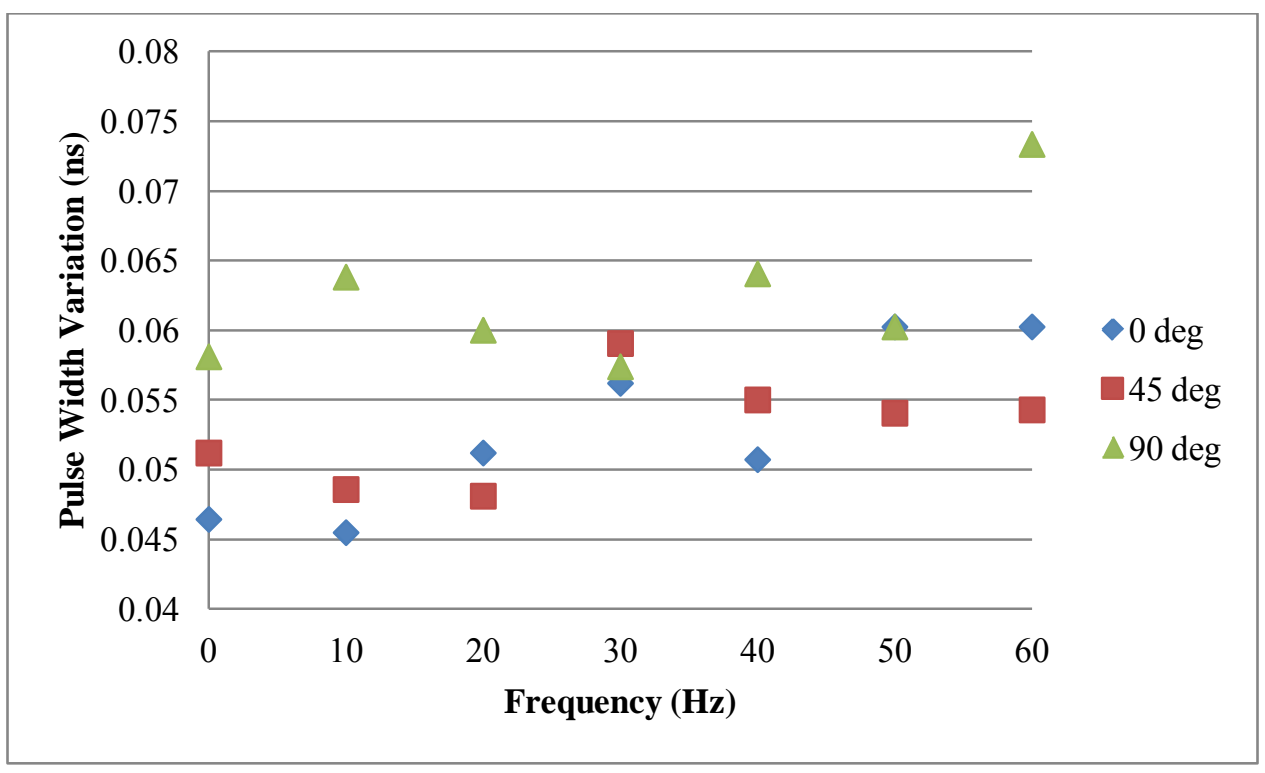

Figure 90: Pulse width variation as a function of frequency for the 0,45 and 90 degree mounting orientation with $100 \%$ acceleration 


\section{Pulse Width: 100\%}

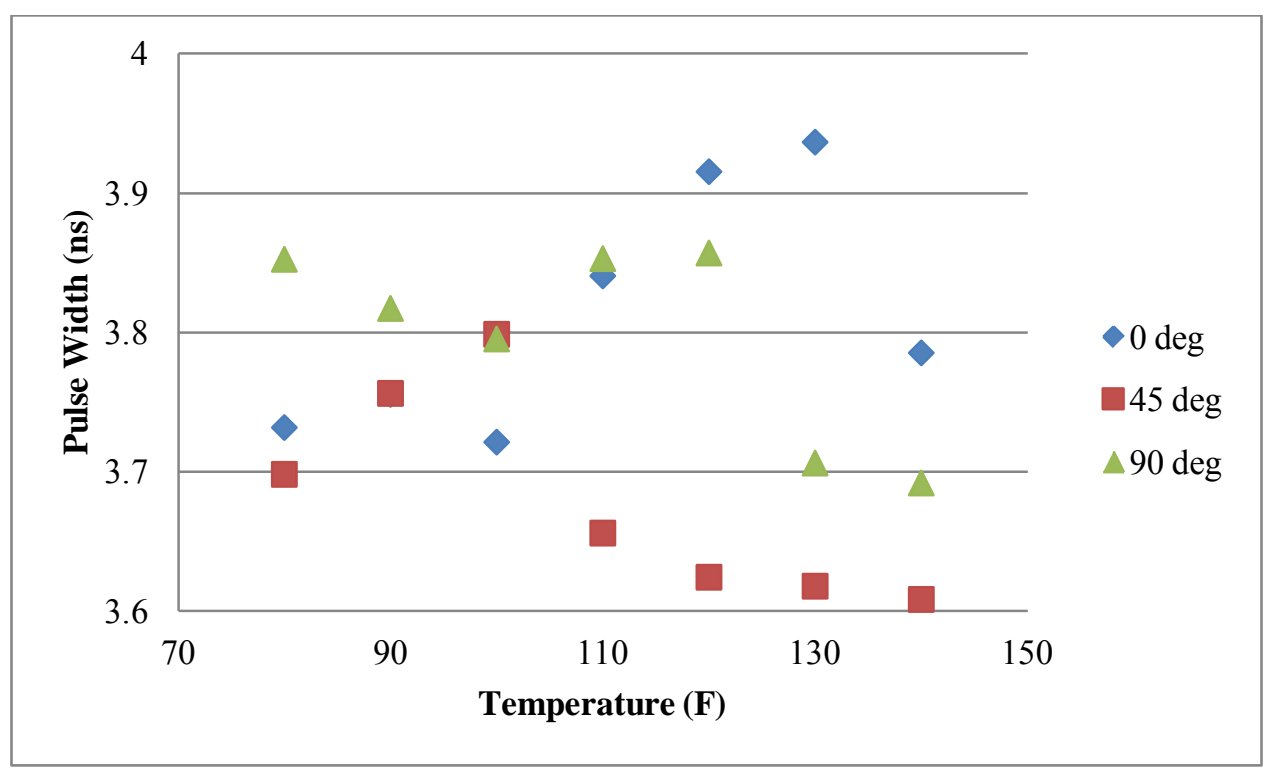

Figure 91: Pulse width as a function of temperature for the 0, 45 and 90 degree mounting orientation with $100 \%$ acceleration

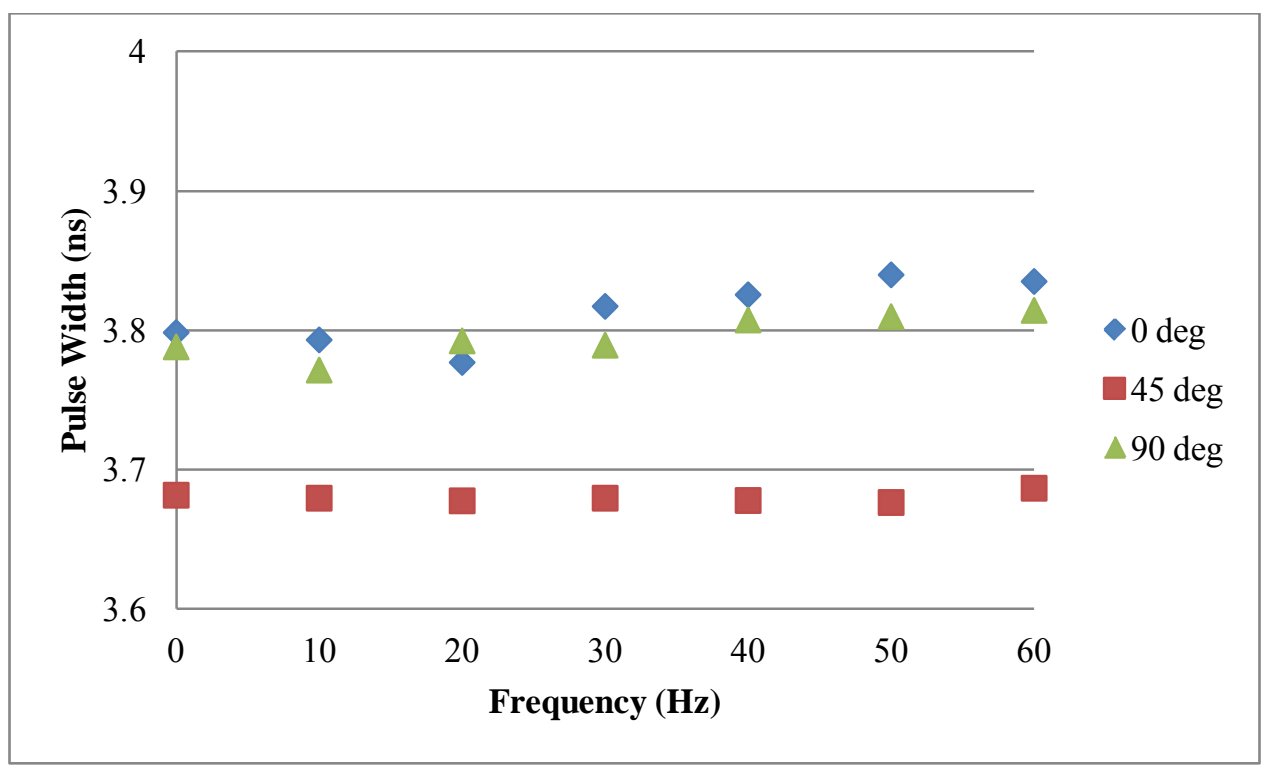

Figure 92: Pulse width as a function of frequency for the 0,45 and 90 degree mounting orientation with $100 \%$ acceleration 


\section{Jitter: 100\%}

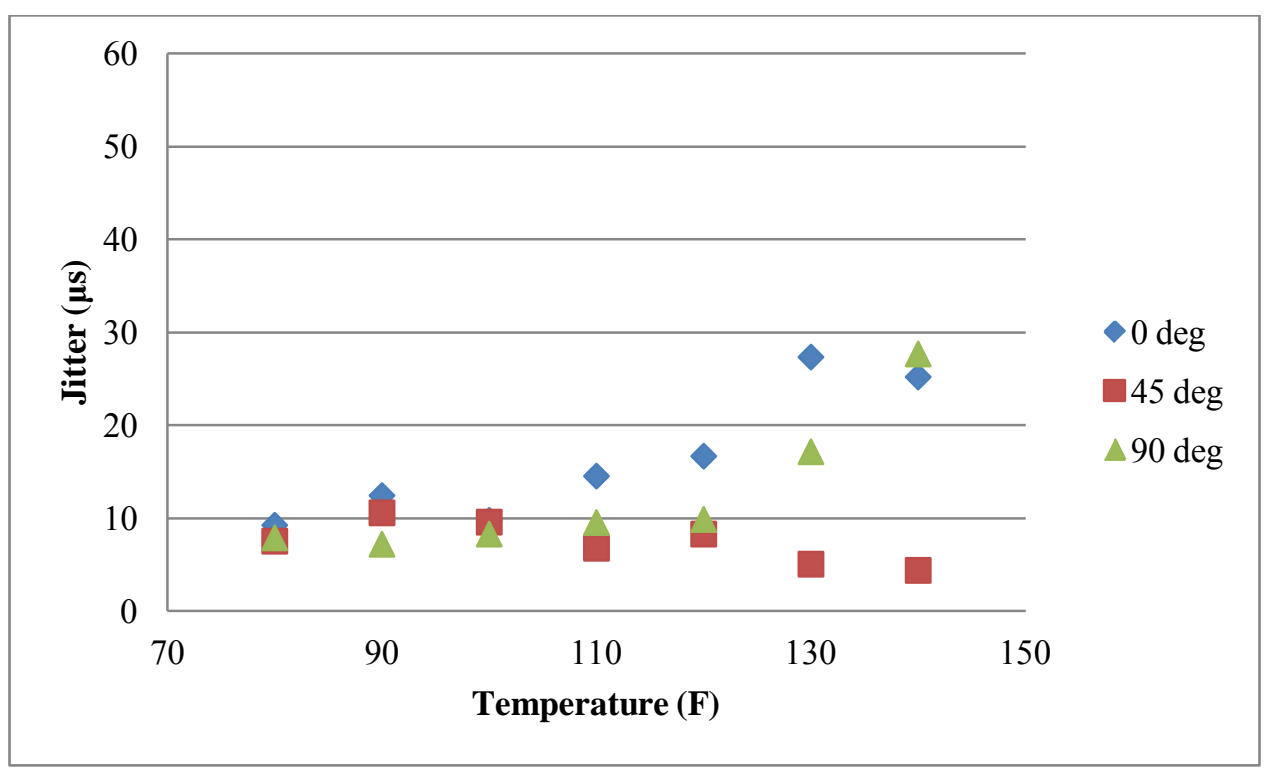

Figure 93: Jitter as a function of temperature for the 0,45 and 90 degree mounting orientation with $100 \%$ acceleration

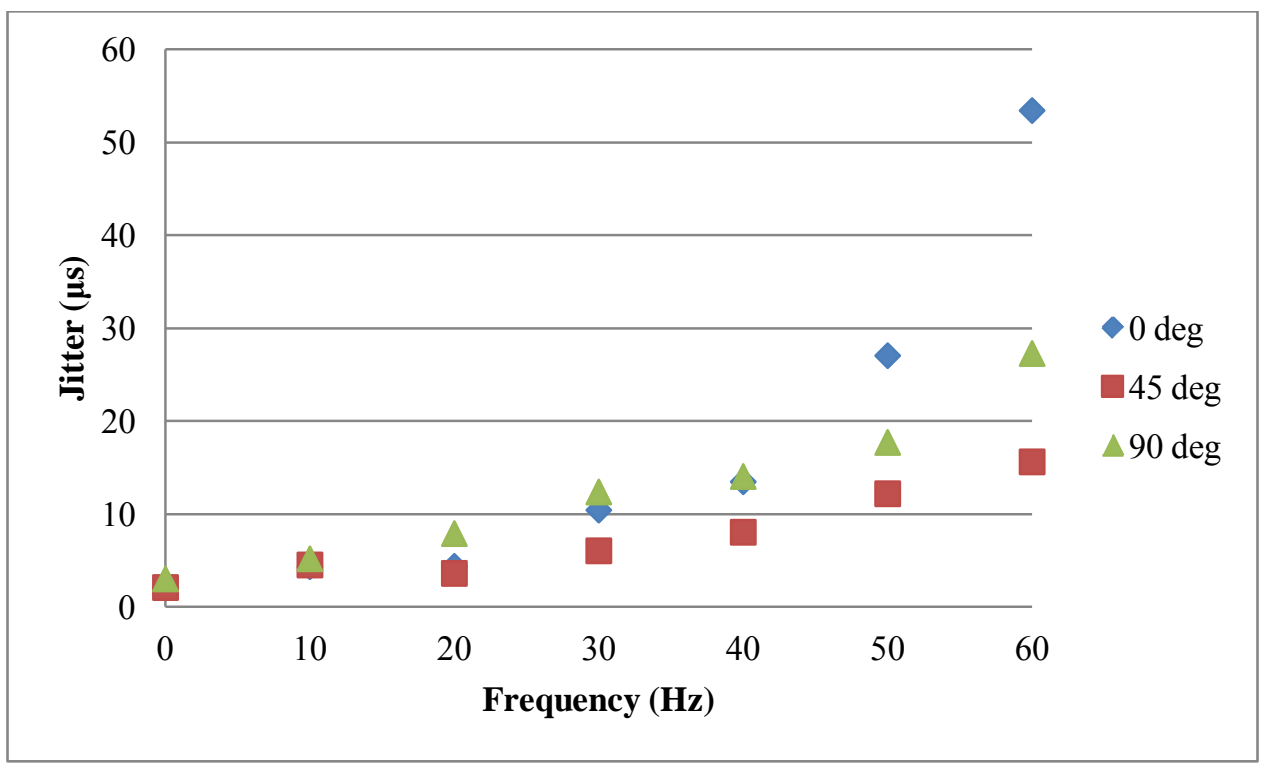

Figure 94: Jitter as a function of frequency for the 0, 45 and 90 degree mounting orientation with $100 \%$ acceleration 


\section{Energy: 100\%}

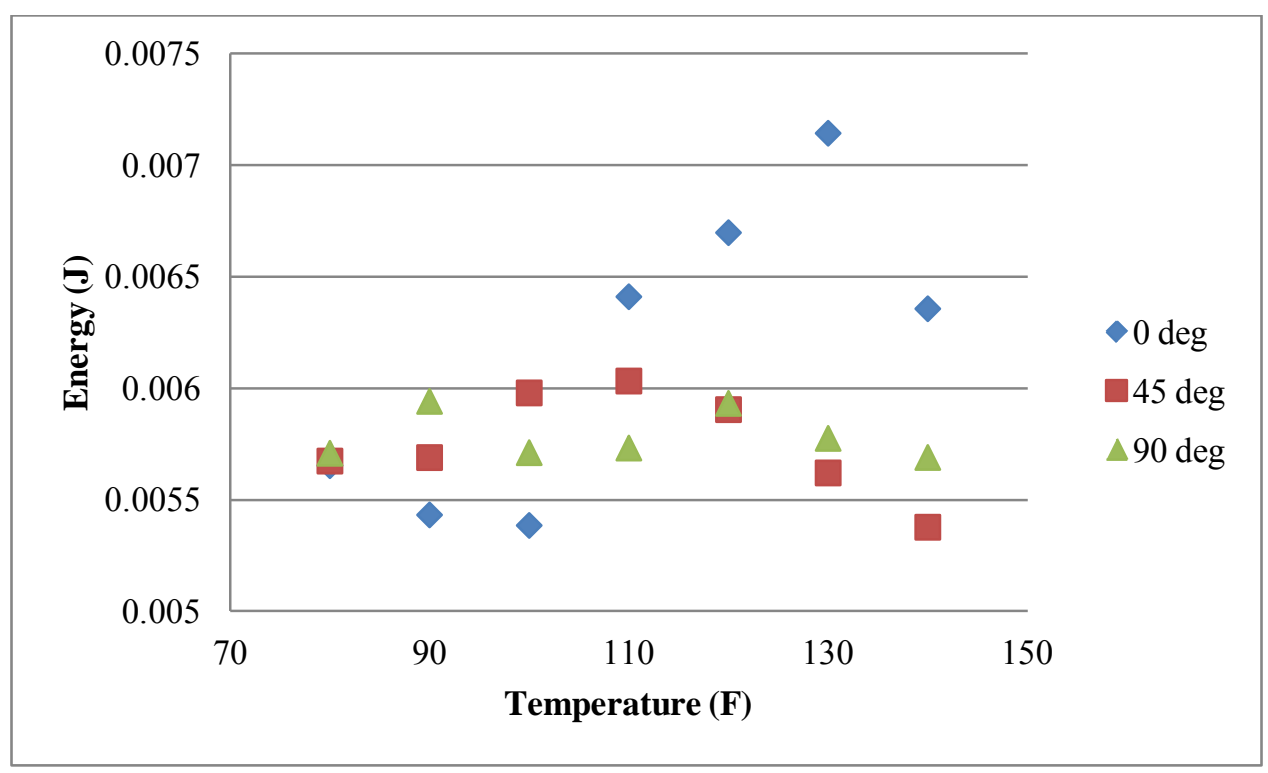

Figure 95: Energy as a function of temperature for the 0, 45 and 90 degree mounting orientations with $100 \%$ acceleration

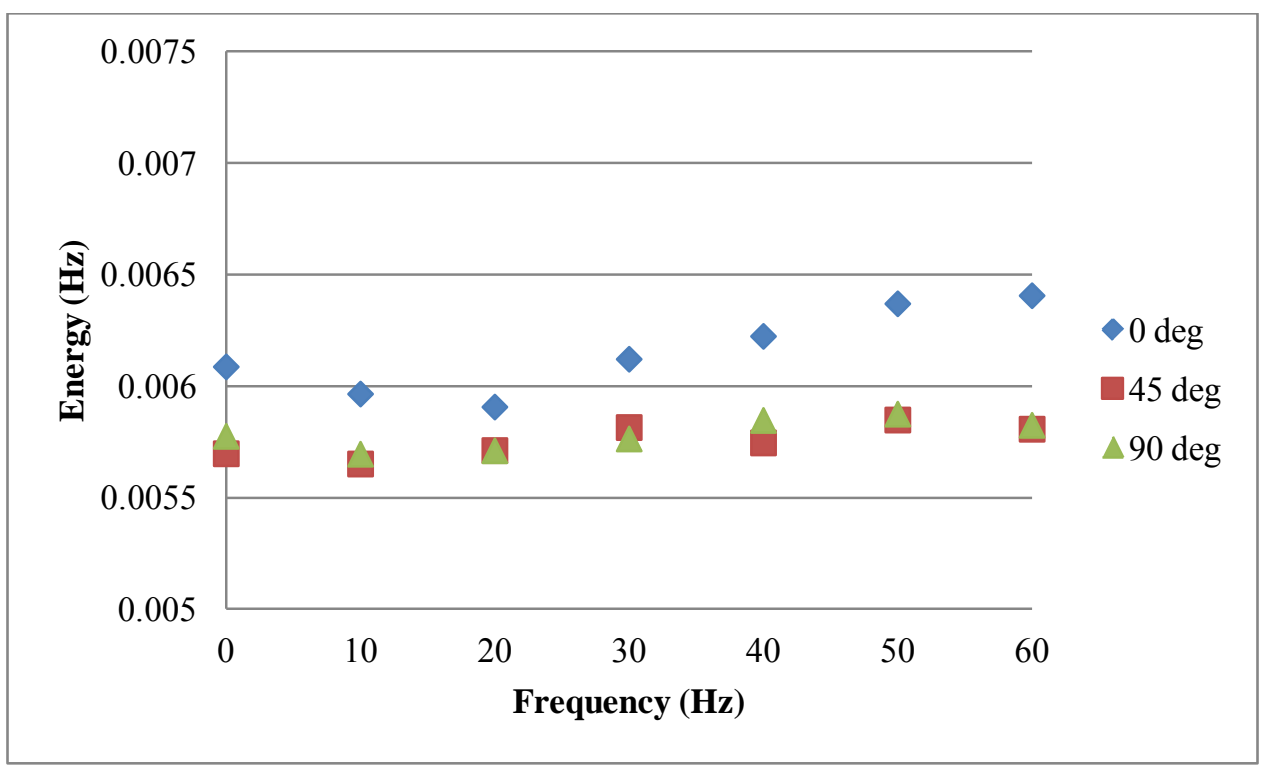

Figure 96: Energy as a function of frequency for the 0,45 and 90 degree mounting orientations with $100 \%$ acceleration 
Focal Intensity: 100\%

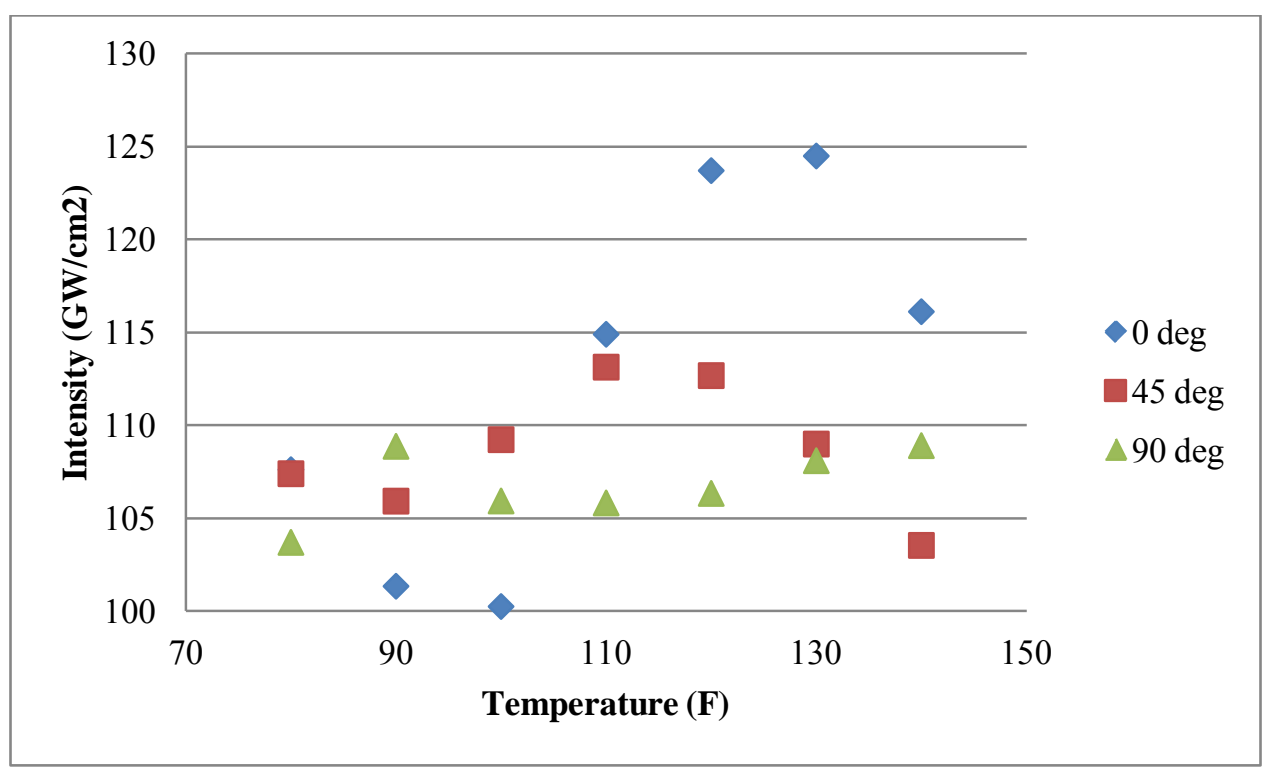

Figure 97: Intensity as a function of temperature for the 0,45 and 90 degree mounting orientations with $100 \%$ acceleration

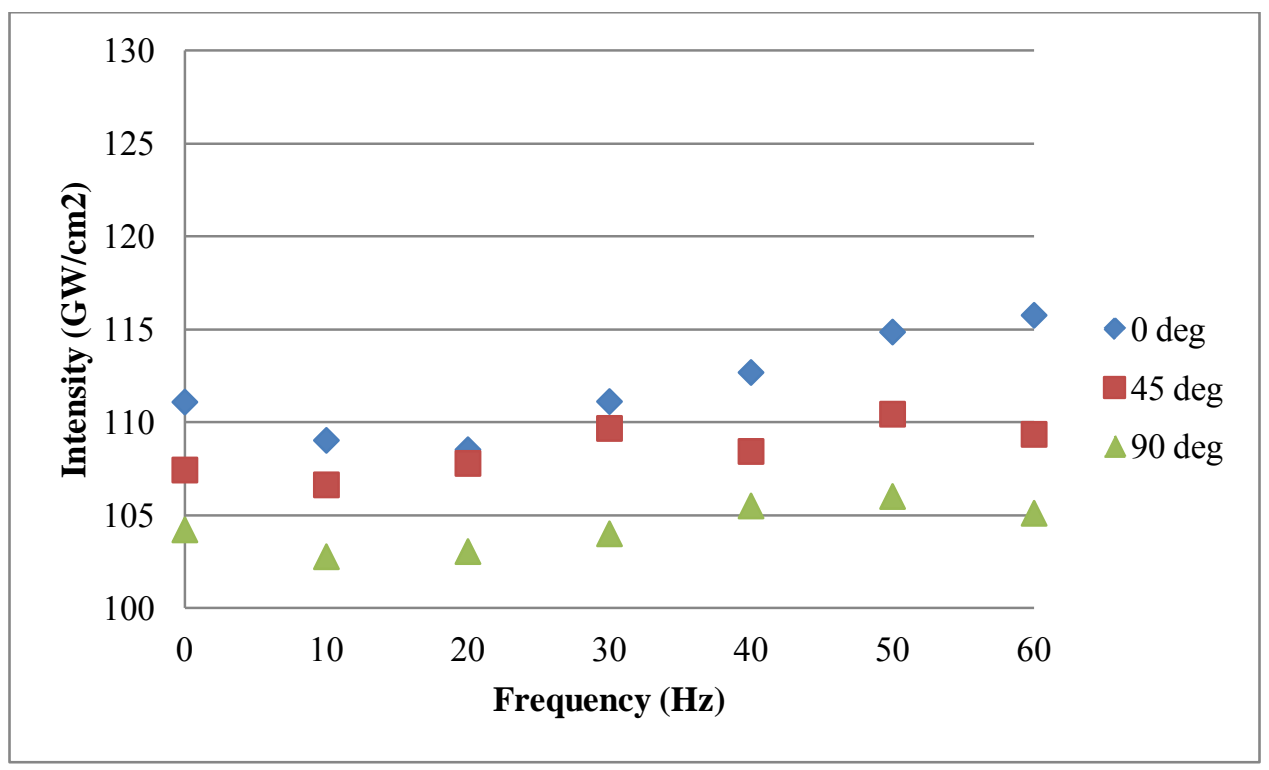

Figure 98: Intensity as a function of frequency for the 0,45 and 90 degree mounting orientations with $100 \%$ acceleration 


\section{Appendix-F: Tabulated Data}

\section{Pulse Width}

Table 7: Pulse width (ns) in the 0 degree mounting orientation with $50 \%$ acceleration

\begin{tabular}{|c|c|c|c|c|c|c|c|c|}
\hline & \multicolumn{7}{|c|}{ Temperature $\left({ }^{\circ} \mathbf{F}\right)$} \\
\hline & & 80 & 90 & 100 & 110 & 120 & 130 & 140 \\
\hline \multirow{7}{*}{ 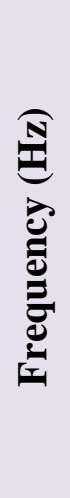 } & 0 & 3.7167 & 3.7617 & 3.7217 & 3.8233 & 3.8600 & 3.9100 & 3.7733 \\
\hline & 10 & 3.7167 & 3.7517 & 3.7000 & 3.8250 & 3.8717 & 3.9050 & 3.7650 \\
\hline & 20 & 3.7300 & 3.7417 & 3.6933 & 3.8483 & 3.9450 & 3.9583 & 3.7667 \\
\hline & 30 & 3.7683 & 3.7683 & 3.7283 & 3.8667 & 3.9233 & 3.9567 & 3.7800 \\
\hline & 40 & 3.7367 & 3.7450 & 3.7267 & 3.8733 & 3.9500 & 3.9467 & 3.8183 \\
\hline & 50 & 3.7767 & 3.7350 & 3.7150 & 3.8683 & 3.9267 & 4.0083 & 3.8333 \\
\hline & 60 & 3.7733 & 3.7717 & 3.7400 & 3.9133 & 3.9300 & 3.8867 & 3.8167 \\
\hline
\end{tabular}

Table 8: Pulse width (ns) in the 0 degree mounting orientation with $100 \%$ acceleration

\begin{tabular}{|c|c|c|c|c|c|c|c|c|}
\hline & \multicolumn{7}{|c|}{ Temperature $\left({ }^{\circ} \mathbf{F}\right)$} \\
\hline & & 80 & 90 & 100 & 110 & 120 & 130 & 140 \\
\hline \multirow{7}{*}{ 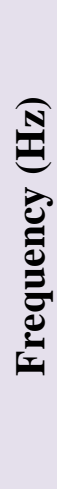 } & 0 & 3.7550 & 3.7383 & 3.7133 & 3.8283 & 3.8533 & 3.9400 & 3.7600 \\
\hline & 10 & 3.7217 & 3.7550 & 3.7100 & 3.8517 & 3.8400 & 3.9150 & 3.7583 \\
\hline & 20 & 3.6917 & 3.7533 & 3.6667 & 3.7700 & 3.9567 & 3.8583 & 3.7417 \\
\hline & 30 & 3.7333 & 3.7583 & 3.7233 & 3.8483 & 3.9300 & 3.9467 & 3.7800 \\
\hline & 40 & 3.7283 & 3.7467 & 3.7483 & 3.8150 & 3.9250 & 3.9900 & 3.8250 \\
\hline & 50 & 3.7250 & 3.7700 & 3.7300 & 3.8833 & 3.9400 & 4.0383 & 3.7917 \\
\hline & 60 & 3.7667 & 3.7667 & 3.7567 & 3.8867 & 3.9617 & 3.8667 & 3.8400 \\
\hline
\end{tabular}


Table 9: Pulse width (ns) in the 45 degree mounting orientation with $50 \%$ acceleration

\begin{tabular}{|c|c|c|c|c|c|c|c|c|}
\hline & & \multicolumn{7}{|c|}{ Temperature $\left({ }^{\circ} \mathrm{F}\right)$} \\
\hline & & 80 & 90 & 100 & 110 & 120 & 130 & 140 \\
\hline \multirow{7}{*}{ 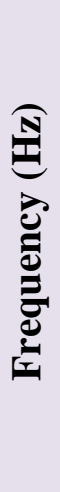 } & $\mathbf{0}$ & 3.6533 & 3.7717 & 3.7933 & 3.6850 & 3.6400 & 3.6367 & 3.6233 \\
\hline & 10 & 3.6817 & 3.7483 & 3.7783 & 3.6750 & 3.6367 & 3.6017 & 3.5967 \\
\hline & 20 & 3.6733 & 3.7833 & 3.8017 & 3.6683 & 3.6500 & 3.6200 & 3.6033 \\
\hline & 30 & 3.7067 & 3.7683 & 3.8067 & 3.6533 & 3.6167 & 3.6017 & 3.6167 \\
\hline & 40 & 3.6550 & 3.7817 & 3.8167 & 3.6500 & 3.6317 & 3.6267 & 3.6050 \\
\hline & 50 & 3.7200 & 3.7783 & 3.8000 & 3.6400 & 3.6367 & 3.6067 & 3.6017 \\
\hline & 60 & 3.6983 & 3.7733 & 3.8217 & 3.6417 & 3.6000 & 3.6183 & 3.5967 \\
\hline
\end{tabular}

Table 10: Pulse width (ns) in the 45 degree mounting orientation with $100 \%$ acceleration

\begin{tabular}{|c|c|c|c|c|c|c|c|c|}
\hline & & \multicolumn{7}{|c|}{ Temperature $\left({ }^{\circ} \mathrm{F}\right)$} \\
\hline & & 80 & 90 & 100 & 110 & 120 & 130 & 140 \\
\hline \multirow{7}{*}{ 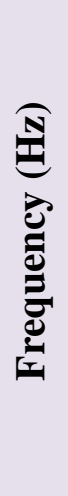 } & $\mathbf{0}$ & 3.6700 & 3.7700 & 3.8017 & 3.6583 & 3.6383 & 3.6233 & 3.6100 \\
\hline & 10 & 3.7000 & 3.7333 & 3.7883 & 3.6800 & 3.6250 & 3.6050 & 3.6250 \\
\hline & 20 & 3.7067 & 3.7417 & 3.8050 & 3.6817 & 3.6183 & 3.6100 & 3.5800 \\
\hline & 30 & 3.7033 & 3.7400 & 3.8100 & 3.6467 & 3.6400 & 3.6267 & 3.5900 \\
\hline & 40 & 3.6767 & 3.7700 & 3.7950 & 3.6467 & 3.6117 & 3.6183 & 3.6267 \\
\hline & 50 & 3.7067 & 3.7767 & 3.8017 & 3.6300 & 3.6067 & 3.6100 & 3.6033 \\
\hline & 60 & 3.7233 & 3.7617 & 3.7883 & 3.6483 & 3.6300 & 3.6317 & 3.6233 \\
\hline
\end{tabular}


Table 11: Pulse width (ns) in the 90 degree mounting orientation with $50 \%$ acceleration

\begin{tabular}{|c|c|c|c|c|c|c|c|c|}
\hline & & \multicolumn{7}{|c|}{ Temperature $\left({ }^{\circ} \mathrm{F}\right)$} \\
\hline & & 80 & 90 & 100 & 110 & 120 & 130 & 140 \\
\hline \multirow{7}{*}{ 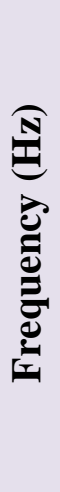 } & $\mathbf{0}$ & 3.8550 & 3.7900 & 3.7717 & 3.8117 & 3.8917 & 3.6700 & 3.6683 \\
\hline & 10 & 3.8583 & 3.8217 & 3.7900 & 3.8433 & 3.8800 & 3.6483 & 3.6533 \\
\hline & 20 & 3.8583 & 3.8183 & 3.7783 & 3.8567 & 3.8850 & 3.6800 & 3.6783 \\
\hline & 30 & 3.8433 & 3.8050 & 3.7917 & 3.8667 & 3.8700 & 3.7083 & 3.7133 \\
\hline & 40 & 3.8483 & 3.8417 & 3.8100 & 3.8333 & 3.8700 & 3.7400 & 3.7100 \\
\hline & 50 & 3.8550 & 3.8583 & 3.8150 & 3.8183 & 3.9000 & 3.7517 & 3.7183 \\
\hline & 60 & 3.8450 & 3.8367 & 3.7883 & 3.8400 & 3.8733 & 3.7767 & 3.7133 \\
\hline
\end{tabular}

Table 12: Pulse width (ns) in the 90 degree mounting orientation with $100 \%$ acceleration

\begin{tabular}{|c|c|c|c|c|c|c|c|c|}
\hline & & \multicolumn{7}{|c|}{ Temperature $\left({ }^{\circ} \mathrm{F}\right)$} \\
\hline & & 80 & 90 & 100 & 110 & 120 & 130 & 140 \\
\hline \multirow{7}{*}{ 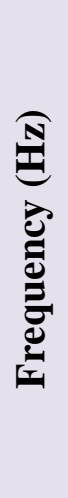 } & $\mathbf{0}$ & 3.8467 & 3.8067 & 3.8067 & 3.8617 & 3.8533 & 3.6850 & 3.6567 \\
\hline & 10 & 3.8267 & 3.8183 & 3.7933 & 3.8467 & 3.8267 & 3.6517 & 3.6367 \\
\hline & 20 & 3.8617 & 3.8217 & 3.7900 & 3.8467 & 3.8583 & 3.7050 & 3.6633 \\
\hline & 30 & 3.8633 & 3.8200 & 3.7883 & 3.8500 & 3.8333 & 3.7000 & 3.6700 \\
\hline & 40 & 3.8567 & 3.8267 & 3.8083 & 3.8417 & 3.8883 & 3.7100 & 3.7200 \\
\hline & 50 & 3.8700 & 3.8200 & 3.7767 & 3.8683 & 3.8683 & 3.7117 & 3.7533 \\
\hline & 60 & 3.8417 & 3.8067 & 3.8033 & 3.8550 & 3.8700 & 3.7800 & 3.7433 \\
\hline
\end{tabular}




\section{Pulse Width Variation}

Table 13: Pulse width variation (ns) in the 0 degree mounting orientation with $50 \%$ acceleration

\begin{tabular}{|c|c|c|c|c|c|c|c|c|}
\hline & \multicolumn{7}{|c|}{ Temperature $\left({ }^{\circ} \mathbf{F}\right)$} \\
\hline & & 80 & 90 & 100 & 110 & 120 & 130 & 140 \\
\hline \multirow{7}{*}{ 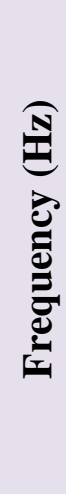 } & $\mathbf{0}$ & 0.0533 & 0.0450 & 0.0517 & 0.0367 & 0.0367 & 0.0600 & 0.0467 \\
\hline & 10 & 0.0433 & 0.0417 & 0.0500 & 0.0517 & 0.0417 & 0.0550 & 0.0483 \\
\hline & 20 & 0.0567 & 0.0550 & 0.0433 & 0.0517 & 0.0317 & 0.0383 & 0.0467 \\
\hline & 30 & 0.0517 & 0.0517 & 0.0450 & 0.0533 & 0.0433 & 0.0400 & 0.0867 \\
\hline & 40 & 0.0567 & 0.0517 & 0.0467 & 0.0400 & 0.0533 & 0.0467 & 0.0550 \\
\hline & 50 & 0.0567 & 0.0517 & 0.0317 & 0.0417 & 0.0600 & 0.0450 & 0.0500 \\
\hline & 60 & 0.0667 & 0.0750 & 0.0367 & 0.0467 & 0.0367 & 0.0600 & 0.0567 \\
\hline
\end{tabular}

Table 14: Pulse width variation (ns) in the 0 degree mounting orientation with $100 \%$ acceleration

\begin{tabular}{|c|c|c|c|c|c|c|c|c|}
\hline & \multicolumn{7}{|c|}{ Temperature $\left({ }^{\circ} \mathbf{F}\right)$} \\
\hline & & 80 & 90 & 100 & 110 & 120 & 130 & 140 \\
\hline \multirow{7}{*}{ 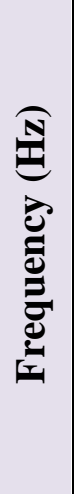 } & $\mathbf{0}$ & 0.0450 & 0.0417 & 0.0600 & 0.0483 & 0.0433 & 0.0400 & 0.0467 \\
\hline & 10 & 0.0483 & 0.0450 & 0.0367 & 0.0417 & 0.0600 & 0.0417 & 0.0450 \\
\hline & 20 & 0.0683 & 0.0533 & 0.0433 & 0.0433 & 0.0433 & 0.0550 & 0.0517 \\
\hline & 30 & 0.0667 & 0.0450 & 0.0500 & 0.0583 & 0.0467 & 0.0667 & 0.0600 \\
\hline & 40 & 0.0517 & 0.0500 & 0.0517 & 0.0450 & 0.0550 & 0.0367 & 0.0650 \\
\hline & 50 & 0.0483 & 0.0633 & 0.0633 & 0.0500 & 0.0600 & 0.0783 & 0.0583 \\
\hline & 60 & 0.0767 & 0.0667 & 0.0733 & 0.0600 & 0.0450 & 0.0400 & 0.0600 \\
\hline
\end{tabular}


Table 15: Pulse width variation (ns) in the 45 degree mounting orientation with $50 \%$ acceleration

\begin{tabular}{|c|c|c|c|c|c|c|c|c|}
\hline & & \multicolumn{7}{|c|}{ Temperature $\left({ }^{\circ} \mathrm{F}\right)$} \\
\hline & & 80 & 90 & 100 & 110 & 120 & 130 & 140 \\
\hline \multirow{7}{*}{ 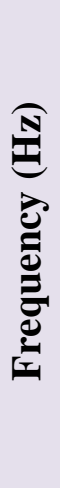 } & $\mathbf{0}$ & 0.0600 & 0.0383 & 0.0400 & 0.0550 & 0.0700 & 0.0733 & 0.0700 \\
\hline & 10 & 0.0450 & 0.0383 & 0.0383 & 0.0650 & 0.0533 & 0.0483 & 0.0633 \\
\hline & 20 & 0.0467 & 0.0567 & 0.0317 & 0.0517 & 0.0567 & 0.0533 & 0.0500 \\
\hline & 30 & 0.0667 & 0.0483 & 0.0400 & 0.0533 & 0.0433 & 0.0583 & 0.0633 \\
\hline & 40 & 0.0583 & 0.0583 & 0.0533 & 0.0800 & 0.0517 & 0.0533 & 0.0650 \\
\hline & 50 & 0.0600 & 0.0483 & 0.0533 & 0.0600 & 0.0567 & 0.0633 & 0.0583 \\
\hline & 60 & 0.0483 & 0.0467 & 0.0517 & 0.0650 & 0.0500 & 0.0683 & 0.0467 \\
\hline
\end{tabular}

Table 16: Pulse width variation (ns) in the 45 degree mounting orientation with $100 \%$ acceleration

\begin{tabular}{|c|c|c|c|c|c|c|c|c|}
\hline & & \multicolumn{7}{|c|}{ Temperature $\left({ }^{\circ} \mathrm{F}\right)$} \\
\hline & & 80 & 90 & 100 & 110 & 120 & 130 & 140 \\
\hline \multirow{7}{*}{ 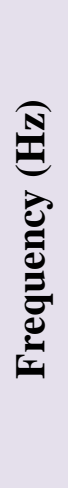 } & $\mathbf{0}$ & 0.0567 & 0.0333 & 0.0450 & 0.0583 & 0.0517 & 0.0633 & 0.0500 \\
\hline & 10 & 0.0533 & 0.0400 & 0.0450 & 0.0533 & 0.0550 & 0.0483 & 0.0450 \\
\hline & 20 & 0.0467 & 0.0417 & 0.0483 & 0.0617 & 0.0417 & 0.0433 & 0.0533 \\
\hline & 30 & 0.0633 & 0.0567 & 0.0500 & 0.0533 & 0.0667 & 0.0600 & 0.0633 \\
\hline & 40 & 0.0500 & 0.0433 & 0.0450 & 0.0733 & 0.0483 & 0.0683 & 0.0567 \\
\hline & 50 & 0.0467 & 0.0500 & 0.0383 & 0.0700 & 0.0533 & 0.0567 & 0.0633 \\
\hline & 60 & 0.0633 & 0.0617 & 0.0450 & 0.0550 & 0.0433 & 0.0550 & 0.0567 \\
\hline
\end{tabular}


Table 17: Pulse width variation (ns) in the 90 degree mounting orientation with $50 \%$ acceleration

\begin{tabular}{|c|c|c|c|c|c|c|c|c|}
\hline & & \multicolumn{7}{|c|}{ Temperature $\left({ }^{\circ} \mathrm{F}\right)$} \\
\hline & & 80 & 90 & 100 & 110 & 120 & 130 & 140 \\
\hline \multirow{7}{*}{ 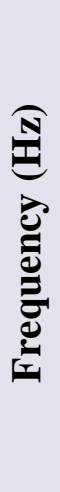 } & $\mathbf{0}$ & 0.0450 & 0.0667 & 0.0483 & 0.0783 & 0.0583 & 0.0567 & 0.0450 \\
\hline & 10 & 0.0517 & 0.0683 & 0.0533 & 0.0633 & 0.0600 & 0.0517 & 0.0533 \\
\hline & 20 & 0.0417 & 0.0583 & 0.0450 & 0.0600 & 0.0617 & 0.0533 & 0.0450 \\
\hline & 30 & 0.0433 & 0.0750 & 0.0817 & 0.0833 & 0.0767 & 0.0750 & 0.0533 \\
\hline & 40 & 0.0450 & 0.0683 & 0.0700 & 0.0767 & 0.0633 & 0.0600 & 0.0567 \\
\hline & 50 & 0.0583 & 0.0550 & 0.0583 & 0.0717 & 0.0600 & 0.0550 & 0.0517 \\
\hline & 60 & 0.0583 & 0.0633 & 0.0883 & 0.0600 & 0.0600 & 0.0500 & 0.0533 \\
\hline
\end{tabular}

Table 18: Pulse width variation (ns) in the 90 degree mounting orientation with $100 \%$ acceleration

\begin{tabular}{|c|c|c|c|c|c|c|c|c|}
\hline & & \multicolumn{7}{|c|}{ Temperature $\left({ }^{\circ} \mathrm{F}\right)$} \\
\hline & & 80 & 90 & 100 & 110 & 120 & 130 & 140 \\
\hline \multirow{7}{*}{ 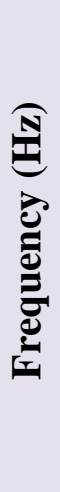 } & $\mathbf{0}$ & 0.0433 & 0.0533 & 0.0767 & 0.0817 & 0.0600 & 0.0483 & 0.0433 \\
\hline & 10 & 0.0667 & 0.0550 & 0.0700 & 0.0667 & 0.0867 & 0.0517 & 0.0500 \\
\hline & 20 & 0.0583 & 0.0550 & 0.0633 & 0.0633 & 0.0817 & 0.0483 & 0.0500 \\
\hline & 30 & 0.0567 & 0.0667 & 0.0650 & 0.0633 & 0.0400 & 0.0600 & 0.0500 \\
\hline & 40 & 0.0533 & 0.0667 & 0.0583 & 0.0850 & 0.0683 & 0.0633 & 0.0533 \\
\hline & 50 & 0.0500 & 0.0833 & 0.0633 & 0.0750 & 0.0617 & 0.0483 & 0.0400 \\
\hline & 60 & 0.0750 & 0.0933 & 0.0900 & 0.0950 & 0.0700 & 0.0533 & 0.0367 \\
\hline
\end{tabular}




\section{Q-Switch Delay}

Table 19: Q-switch delay $(\mu \mathrm{s})$ in the 0 degree mounting orientation with $50 \%$ acceleration

\begin{tabular}{|c|c|c|c|c|c|c|c|c|}
\hline & \multicolumn{7}{|c|}{ Temperature $\left({ }^{\circ} \mathbf{F}\right)$} \\
\hline & & 80 & 90 & 100 & 110 & 120 & 130 & 140 \\
\hline \multirow{7}{*}{ 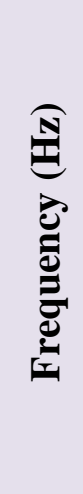 } & $\mathbf{0}$ & 257.33 & 268.67 & 265.33 & 284.67 & 307.33 & 326.67 & 305.00 \\
\hline & 10 & 249.67 & 263.67 & 263.33 & 282.33 & 309.33 & 329.67 & 301.33 \\
\hline & 20 & 255.33 & 273.67 & 260.67 & 292.33 & 338.33 & 346.00 & 307.33 \\
\hline & 30 & 265.00 & 280.67 & 262.33 & 300.67 & 354.67 & 378.33 & 321.33 \\
\hline & 40 & 272.33 & 290.00 & 265.67 & 325.00 & 376.33 & 397.33 & 349.00 \\
\hline & 50 & 279.67 & 299.67 & 272.67 & 351.67 & 412.00 & 475.33 & 392.67 \\
\hline & 60 & 288.00 & 316.33 & 279.33 & 386.67 & 437.33 & 458.00 & 460.33 \\
\hline
\end{tabular}

Table 20: Q-switch delay ( $\mu \mathrm{s})$ in the 0 degree mounting orientation with $100 \%$ acceleration

\begin{tabular}{|c|c|c|c|c|c|c|c|c|}
\hline & \multicolumn{7}{|c|}{ Temperature $\left({ }^{\circ} \mathbf{F}\right)$} \\
\hline & & 80 & 90 & 100 & 110 & 120 & 130 & 140 \\
\hline \multirow{7}{*}{ 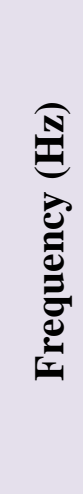 } & $\mathbf{0}$ & 255.67 & 271.67 & 262.67 & 294.67 & 310.67 & 336.67 & 301.00 \\
\hline & 10 & 253.00 & 270.67 & 262.33 & 291.33 & 313.00 & 340.33 & 299.67 \\
\hline & 20 & 243.33 & 279.00 & 258.33 & 299.00 & 334.67 & 338.67 & 305.67 \\
\hline & 30 & 249.33 & 275.67 & 265.67 & 312.67 & 338.33 & 402.00 & 318.00 \\
\hline & 40 & 264.33 & 283.33 & 269.67 & 323.33 & 392.00 & 431.67 & 338.00 \\
\hline & 50 & 268.67 & 298.00 & 279.00 & 369.67 & 390.00 & 450.67 & 398.00 \\
\hline & 60 & 276.33 & 321.33 & 290.00 & 405.67 & 407.33 & 406.00 & 434.00 \\
\hline
\end{tabular}


Table 21: Q-switch delay $(\mu \mathrm{s})$ in the 45 degree mounting orientation with $50 \%$ acceleration

\begin{tabular}{|c|c|c|c|c|c|c|c|c|}
\hline & & \multicolumn{7}{|c|}{ Temperature $\left({ }^{\circ} \mathrm{F}\right)$} \\
\hline & & 80 & 90 & 100 & 110 & 120 & 130 & 140 \\
\hline \multirow{7}{*}{ 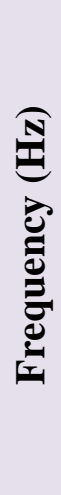 } & $\mathbf{0}$ & 248.33 & 243.33 & 249.00 & 249.00 & 244.00 & 252.67 & 241.33 \\
\hline & 10 & 249.00 & 239.33 & 245.67 & 247.67 & 241.00 & 248.00 & 239.33 \\
\hline & 20 & 246.00 & 242.67 & 247.33 & 246.67 & 238.00 & 243.67 & 235.00 \\
\hline & 30 & 250.33 & 245.67 & 250.67 & 240.00 & 233.67 & 242.33 & 233.00 \\
\hline & 40 & 254.00 & 248.67 & 253.67 & 240.67 & 233.33 & 240.67 & 235.00 \\
\hline & 50 & 259.00 & 249.67 & 261.00 & 237.00 & 233.67 & 242.33 & 238.00 \\
\hline & 60 & 264.67 & 254.00 & 260.67 & 238.33 & 236.00 & 241.67 & 241.00 \\
\hline
\end{tabular}

Table 22: Q-switch delay ( $\mu \mathrm{s})$ in the 45 degree mounting orientation with $100 \%$ acceleration

\begin{tabular}{|c|c|c|c|c|c|c|c|c|}
\hline & & \multicolumn{7}{|c|}{ Temperature $\left({ }^{\circ} \mathrm{F}\right)$} \\
\hline & & 80 & 90 & 100 & 110 & 120 & 130 & 140 \\
\hline \multirow{7}{*}{ 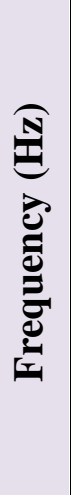 } & $\mathbf{0}$ & 245.00 & 246.00 & 250.00 & 246.67 & 245.67 & 250.33 & 245.00 \\
\hline & 10 & 244.67 & 243.33 & 246.67 & 246.33 & 242.33 & 245.33 & 242.33 \\
\hline & 20 & 246.00 & 243.33 & 247.00 & 244.67 & 238.33 & 242.00 & 235.33 \\
\hline & 30 & 248.33 & 242.67 & 251.33 & 240.67 & 235.00 & 238.67 & 234.33 \\
\hline & 40 & 251.33 & 245.00 & 255.00 & 240.00 & 233.33 & 238.00 & 235.67 \\
\hline & 50 & 255.00 & 245.67 & 253.33 & 239.67 & 239.00 & 241.33 & 240.00 \\
\hline & 60 & 262.33 & 251.33 & 260.33 & 239.33 & 238.33 & 243.00 & 240.67 \\
\hline
\end{tabular}


Table 23: Q-switch delay $(\mu \mathrm{s})$ in the 90 degree mounting orientation with $50 \%$ acceleration

\begin{tabular}{|c|c|c|c|c|c|c|c|c|}
\hline & & \multicolumn{7}{|c|}{ Temperature $\left({ }^{\circ} \mathrm{F}\right)$} \\
\hline & & 80 & 90 & 100 & 110 & 120 & 130 & 140 \\
\hline \multirow{7}{*}{ 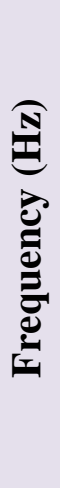 } & $\mathbf{0}$ & 251.33 & 255.00 & 258.33 & 273.00 & 283.33 & 309.33 & 328.67 \\
\hline & 10 & 249.67 & 254.33 & 253.00 & 273.00 & 282.67 & 303.67 & 323.00 \\
\hline & 20 & 249.33 & 255.67 & 255.00 & 272.00 & 277.33 & 306.33 & 325.67 \\
\hline & 30 & 249.33 & 256.00 & 258.00 & 272.33 & 279.67 & 315.67 & 342.00 \\
\hline & 40 & 250.67 & 261.67 & 258.67 & 273.67 & 283.00 & 331.67 & 359.33 \\
\hline & 50 & 254.00 & 264.00 & 263.33 & 279.33 & 286.67 & 339.00 & 379.67 \\
\hline & 60 & 256.67 & 268.67 & 267.33 & 282.00 & 289.33 & 349.67 & 401.67 \\
\hline
\end{tabular}

Table 24: Q-switch delay ( $\mu$ s) in the 90 degree mounting orientation with $100 \%$ acceleration

\begin{tabular}{|c|c|c|c|c|c|c|c|c|}
\hline & & \multicolumn{7}{|c|}{ Temperature $\left({ }^{\circ} \mathrm{F}\right)$} \\
\hline & & 80 & 90 & 100 & 110 & 120 & 130 & 140 \\
\hline \multirow{7}{*}{ 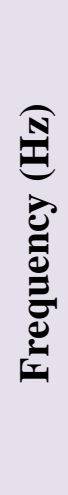 } & $\mathbf{0}$ & 254.83 & 267.33 & 257.00 & 274.00 & 279.00 & 301.33 & 321.00 \\
\hline & 10 & 247.67 & 260.33 & 254.33 & 268.00 & 278.00 & 304.00 & 318.67 \\
\hline & 20 & 248.67 & 260.33 & 256.33 & 267.67 & 276.00 & 302.33 & 330.67 \\
\hline & 30 & 251.67 & 259.67 & 258.33 & 267.33 & 281.67 & 312.67 & 341.33 \\
\hline & 40 & 254.33 & 260.33 & 262.00 & 272.00 & 281.33 & 341.67 & 358.00 \\
\hline & 50 & 256.00 & 263.67 & 264.00 & 275.33 & 283.33 & 338.00 & 390.33 \\
\hline & 60 & 257.33 & 266.67 & 266.00 & 278.33 & 286.33 & 348.67 & 442.33 \\
\hline
\end{tabular}




\section{$\underline{\text { Jitter }}$}

Table 25: Jitter $(\mu \mathrm{s})$ in the 0 degree mounting orientation with $50 \%$ acceleration

\begin{tabular}{|c|c|c|c|c|c|c|c|c|}
\hline & & \multicolumn{7}{|c|}{ Temperature $\left({ }^{\circ} \mathrm{F}\right)$} \\
\hline & & 80 & 90 & 100 & 110 & 120 & 130 & 140 \\
\hline \multirow{7}{*}{ 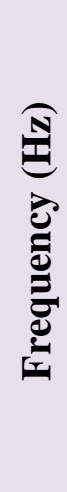 } & $\mathbf{0}$ & 0.667 & 1.333 & 1.333 & 2.000 & 2.667 & 4.000 & 3.000 \\
\hline & 10 & 1.667 & 1.667 & 1.333 & 2.333 & 3.333 & 4.333 & 4.000 \\
\hline & 20 & 2.667 & 3.000 & 2.000 & 3.000 & 4.333 & 6.000 & 5.333 \\
\hline & 30 & 5.000 & 3.333 & 3.667 & 6.000 & 6.000 & 9.000 & 8.000 \\
\hline & 40 & 3.667 & 4.667 & 3.667 & 6.333 & 9.667 & 12.667 & 13.667 \\
\hline & 50 & 6.333 & 5.667 & 6.667 & 10.333 & 11.333 & 24.667 & 28.000 \\
\hline & 60 & 11.333 & 11.667 & 8.000 & 20.000 & 12.667 & 42.000 & 83.000 \\
\hline
\end{tabular}

Table 26: Jitter $(\mu \mathrm{s})$ in the 0 degree mounting orientation with $100 \%$ acceleration

\begin{tabular}{|c|c|c|c|c|c|c|c|c|}
\hline & & \multicolumn{7}{|c|}{ Temperature $\left({ }^{\circ} \mathrm{F}\right)$} \\
\hline & & 80 & 90 & 100 & 110 & 120 & 130 & 140 \\
\hline \multirow{7}{*}{ 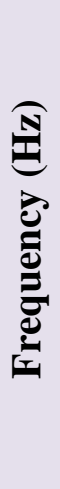 } & $\mathbf{0}$ & 1.00 & 1.00 & 1.33 & 2.00 & 2.67 & 3.33 & 3.00 \\
\hline & 10 & 3.67 & 4.00 & 1.67 & 2.67 & 6.33 & 7.67 & 4.33 \\
\hline & 20 & 2.00 & 2.33 & 3.00 & 3.67 & 7.33 & 6.67 & 5.67 \\
\hline & 30 & 6.67 & 9.67 & 5.67 & 9.33 & 12.33 & 18.00 & 11.33 \\
\hline & 40 & 10.33 & 13.33 & 10.33 & 15.33 & 14.00 & 19.00 & 12.00 \\
\hline & 50 & 16.00 & 26.00 & 15.00 & 24.33 & 31.33 & 42.67 & 34.00 \\
\hline & 60 & 25.00 & 30.67 & 31.33 & 44.33 & 42.67 & 94.00 & 106.00 \\
\hline
\end{tabular}


Table 27: Jitter ( $\mu \mathrm{s})$ in the 45 degree mounting orientation with $50 \%$ acceleration

\begin{tabular}{|c|c|c|c|c|c|c|c|c|}
\hline & \multicolumn{7}{|c|}{ Temperature $\left({ }^{\circ} \mathbf{F}\right)$} \\
\hline & & 80 & 90 & 100 & 110 & 120 & 130 & 140 \\
\hline \multirow{7}{*}{ 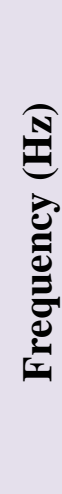 } & $\mathbf{0}$ & 1.67 & 1.33 & 1.67 & 1.67 & 2.00 & 2.00 & 2.00 \\
\hline & 10 & 2.33 & 2.67 & 2.33 & 2.33 & 3.00 & 2.00 & 2.00 \\
\hline & 20 & 2.67 & 2.67 & 2.67 & 3.33 & 2.67 & 3.00 & 3.00 \\
\hline & 30 & 3.67 & 3.67 & 4.00 & 4.67 & 3.00 & 4.33 & 3.67 \\
\hline & 40 & 4.00 & 4.67 & 4.33 & 4.67 & 4.00 & 4.00 & 3.67 \\
\hline & 50 & 5.67 & 9.67 & 8.33 & 6.33 & 5.67 & 5.00 & 4.00 \\
\hline & 60 & 7.33 & 11.33 & 15.33 & 9.67 & 8.67 & 6.33 & 5.67 \\
\hline
\end{tabular}

Table 28: Jitter ( $\mu \mathrm{s})$ in the 45 degree mounting orientation with $100 \%$ acceleration

\begin{tabular}{|c|c|c|c|c|c|c|c|c|}
\hline & \multicolumn{7}{|c|}{ Temperature $\left({ }^{\circ} \mathbf{F}\right)$} \\
\hline & & 80 & 90 & 100 & 110 & 120 & 130 & 140 \\
\hline \multirow{7}{*}{ 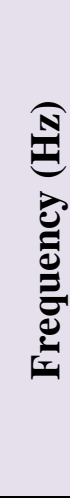 } & $\mathbf{0}$ & 2.33 & 2.00 & 2.00 & 1.33 & 2.33 & 2.33 & 2.33 \\
\hline & 10 & 2.00 & 3.33 & 3.33 & 2.33 & 12.33 & 4.00 & 4.33 \\
\hline & 20 & 3.33 & 3.33 & 3.67 & 2.67 & 5.00 & 4.00 & 3.33 \\
\hline & 30 & 5.00 & 6.67 & 6.00 & 6.00 & 9.00 & 5.33 & 4.33 \\
\hline & 40 & 9.33 & 11.67 & 7.67 & 9.33 & 8.00 & 4.67 & 5.67 \\
\hline & 50 & 14.33 & 21.67 & 17.33 & 11.00 & 9.00 & 7.33 & 4.67 \\
\hline & 60 & 16.33 & 25.33 & 27.00 & 14.67 & 12.33 & 7.67 & 6.00 \\
\hline
\end{tabular}


Table 29: Jitter ( $\mu \mathrm{s})$ in the 90 degree mounting orientation with $50 \%$ acceleration

\begin{tabular}{|c|c|c|c|c|c|c|c|c|}
\hline & \multicolumn{7}{|c|}{ Temperature $\left({ }^{\circ} \mathbf{F}\right)$} \\
\hline & & 80 & 90 & 100 & 110 & 120 & 130 & 140 \\
\hline \multirow{7}{*}{ 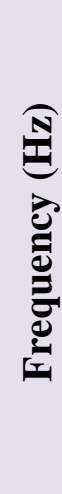 } & $\mathbf{0}$ & 2.00 & 2.33 & 2.33 & 3.67 & 2.67 & 4.00 & 5.33 \\
\hline & 10 & 3.00 & 2.33 & 3.67 & 5.67 & 3.33 & 5.67 & 7.00 \\
\hline & 20 & 4.67 & 3.67 & 4.33 & 6.67 & 4.67 & 10.33 & 10.33 \\
\hline & 30 & 6.00 & 6.00 & 8.00 & 9.00 & 7.00 & 15.00 & 19.33 \\
\hline & 40 & 7.33 & 6.33 & 7.33 & 9.67 & 9.67 & 17.67 & 26.00 \\
\hline & 50 & 7.33 & 6.67 & 8.00 & 10.67 & 9.33 & 24.33 & 33.67 \\
\hline & 60 & 9.33 & 10.00 & 10.67 & 12.67 & 12.67 & 29.00 & 40.33 \\
\hline
\end{tabular}

Table 30: Jitter ( $\mu \mathrm{s})$ in the 90 degree mounting orientation with $100 \%$ acceleration

\begin{tabular}{|c|c|c|c|c|c|c|c|c|}
\hline & \multicolumn{7}{|c|}{ Temperature $\left({ }^{\circ} \mathbf{F}\right)$} \\
\hline & & 80 & 90 & 100 & 110 & 120 & 130 & 140 \\
\hline \multirow{7}{*}{ 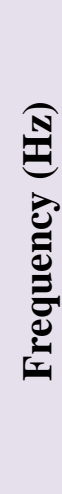 } & $\mathbf{0}$ & 2.83 & 2.00 & 3.00 & 3.33 & 2.33 & 3.33 & 4.33 \\
\hline & 10 & 3.67 & 3.67 & 3.00 & 6.67 & 4.00 & 6.00 & 9.33 \\
\hline & 20 & 6.00 & 5.00 & 5.67 & 7.00 & 7.33 & 10.33 & 14.00 \\
\hline & 30 & 9.00 & 7.67 & 9.00 & 11.33 & 9.67 & 17.33 & 22.67 \\
\hline & 40 & 8.33 & 7.67 & 10.67 & 11.33 & 12.67 & 19.00 & 28.67 \\
\hline & 50 & 10.67 & 10.33 & 12.00 & 12.00 & 14.00 & 27.33 & 37.67 \\
\hline & 60 & 14.67 & 14.00 & 14.67 & 15.00 & 19.00 & 36.67 & 77.00 \\
\hline
\end{tabular}




\section{Energy}

Table 31: Energy $(\mathrm{J})$ in the 0 degree mounting orientation with $50 \%$ acceleration

\begin{tabular}{|c|c|c|c|c|c|c|c|c|}
\hline & \multicolumn{7}{|c|}{ Temperature $\left({ }^{\circ} \mathbf{F}\right)$} \\
\hline & & 80 & 90 & 100 & 110 & 120 & 130 & 140 \\
\hline \multirow{7}{*}{ 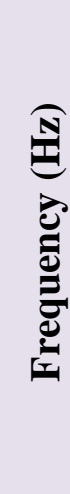 } & $\mathbf{0}$ & 0.00572 & 0.00552 & 0.00537 & 0.00610 & 0.00660 & 0.00670 & 0.00621 \\
\hline & 10 & 0.00556 & 0.00542 & 0.00531 & 0.00600 & 0.00646 & 0.00661 & 0.00614 \\
\hline & 20 & 0.00565 & 0.00547 & 0.00522 & 0.00617 & 0.00665 & 0.00661 & 0.00597 \\
\hline & 30 & 0.00597 & 0.00556 & 0.00543 & 0.00616 & 0.00700 & 0.00728 & 0.00627 \\
\hline & 40 & 0.00571 & 0.00554 & 0.00545 & 0.00654 & 0.00739 & 0.00741 & 0.00630 \\
\hline & 50 & 0.00594 & 0.00543 & 0.00527 & 0.00683 & 0.00741 & 0.00731 & 0.00672 \\
\hline & 60 & 0.00610 & 0.00542 & 0.00554 & 0.00693 & 0.00735 & 0.00752 & 0.00681 \\
\hline
\end{tabular}

Table 32: Energy $(\mathrm{J})$ in the 0 degree mounting orientation with 100\% acceleration

\begin{tabular}{|c|c|c|c|c|c|c|c|c|}
\hline & \multicolumn{7}{|c|}{ Temperature $\left({ }^{\circ} \mathrm{F}\right)$} \\
\hline & & 80 & 90 & 100 & 110 & 120 & 130 & 140 \\
\hline \multirow{7}{*}{ 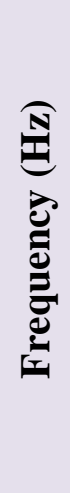 } & o & 0.00574 & 0.00541 & 0.00534 & 0.00610 & 0.00667 & 0.00698 & 0.00636 \\
\hline & 10 & 0.00556 & 0.00532 & 0.00546 & 0.00603 & 0.00637 & 0.00698 & 0.00603 \\
\hline & 20 & 0.00571 & 0.00535 & 0.00524 & 0.00619 & 0.00563 & 0.00697 & 0.00625 \\
\hline & 30 & 0.00558 & 0.00561 & 0.00533 & 0.00619 & 0.00698 & 0.00699 & 0.00616 \\
\hline & 40 & 0.00559 & 0.00545 & 0.00534 & 0.00654 & 0.00686 & 0.00748 & 0.00630 \\
\hline & 50 & 0.00566 & 0.00544 & 0.00539 & 0.00683 & 0.00720 & 0.00748 & 0.00658 \\
\hline & 60 & 0.00572 & 0.00544 & 0.00559 & 0.00698 & 0.00719 & 0.00711 & 0.00681 \\
\hline
\end{tabular}


Table 33: Energy $(\mathrm{J})$ in the 45 degree mounting orientation with $50 \%$ acceleration

\begin{tabular}{|c|c|c|c|c|c|c|c|c|}
\hline & \multicolumn{7}{|c|}{ Temperature $\left({ }^{\circ} \mathbf{F}\right)$} \\
\hline & & 80 & 90 & 100 & 110 & 120 & 130 & 140 \\
\hline \multirow{7}{*}{ 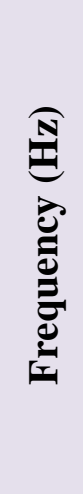 } & 0 & 0.00556 & 0.00552 & 0.00587 & 0.00604 & 0.00614 & 0.00574 & 0.00532 \\
\hline & 10 & 0.00557 & 0.00540 & 0.00582 & 0.00601 & 0.00610 & 0.00566 & 0.00530 \\
\hline & 20 & 0.00565 & 0.00599 & 0.00583 & 0.00592 & 0.00587 & 0.00562 & 0.00534 \\
\hline & 30 & 0.00550 & 0.00597 & 0.00606 & 0.00580 & 0.00572 & 0.00552 & 0.00541 \\
\hline & 40 & 0.00585 & 0.00577 & 0.00619 & 0.00588 & 0.00573 & 0.00567 & 0.00547 \\
\hline & 50 & 0.00591 & 0.00587 & 0.00605 & 0.00605 & 0.00581 & 0.00571 & 0.00540 \\
\hline & 60 & 0.00585 & 0.00578 & 0.00606 & 0.00604 & 0.00588 & 0.00582 & 0.00542 \\
\hline
\end{tabular}

Table 34: Energy $(\mathrm{J})$ in the 45 degree mounting orientation with $100 \%$ acceleration

\begin{tabular}{|c|c|c|c|c|c|c|c|c|}
\hline & \multicolumn{7}{|c|}{ Temperature $\left({ }^{\circ} \mathbf{F}\right)$} \\
\hline & & 80 & 90 & 100 & 110 & 120 & 130 & 140 \\
\hline \multirow{7}{*}{ 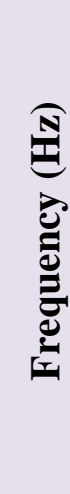 } & $\mathbf{0}$ & 0.00552 & 0.00549 & 0.00588 & 0.00604 & 0.00604 & 0.00563 & 0.00526 \\
\hline & 10 & 0.00545 & 0.00539 & 0.00576 & 0.00605 & 0.00617 & 0.00549 & 0.00524 \\
\hline & 20 & 0.00564 & 0.00591 & 0.00582 & 0.00593 & 0.00581 & 0.00557 & 0.00530 \\
\hline & 30 & 0.00554 & 0.00597 & 0.00617 & 0.00601 & 0.00595 & 0.00555 & 0.00550 \\
\hline & 40 & 0.00577 & 0.00573 & 0.00608 & 0.00590 & 0.00564 & 0.00565 & 0.00543 \\
\hline & 50 & 0.00598 & 0.00565 & 0.00612 & 0.00623 & 0.00584 & 0.00566 & 0.00546 \\
\hline & 60 & 0.00581 & 0.00568 & 0.00602 & 0.00605 & 0.00587 & 0.00579 & 0.00543 \\
\hline
\end{tabular}


Table 35: Energy $(\mathrm{J})$ in the 90 degree mounting orientation with 50\% acceleration

\begin{tabular}{|c|c|c|c|c|c|c|c|c|}
\hline & \multicolumn{7}{|c|}{ Temperature $\left({ }^{\circ} \mathbf{F}\right)$} \\
\hline & & 80 & 90 & 100 & 110 & 120 & 130 & 140 \\
\hline \multirow{7}{*}{ 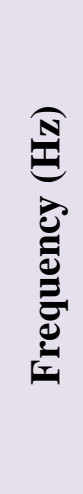 } & 0 & 0.00563 & 0.00571 & 0.00571 & 0.00588 & 0.00605 & 0.00575 & 0.00580 \\
\hline & 10 & 0.00558 & 0.00572 & 0.00571 & 0.00586 & 0.00605 & 0.00569 & 0.00568 \\
\hline & 20 & 0.00576 & 0.00629 & 0.00570 & 0.00571 & 0.00588 & 0.00563 & 0.00576 \\
\hline & 30 & 0.00571 & 0.00622 & 0.00586 & 0.00583 & 0.00584 & 0.00559 & 0.00580 \\
\hline & 40 & 0.00585 & 0.00601 & 0.00589 & 0.00583 & 0.00594 & 0.00596 & 0.00582 \\
\hline & 50 & 0.00598 & 0.00592 & 0.00591 & 0.00598 & 0.00592 & 0.00583 & 0.00589 \\
\hline & 60 & 0.00577 & 0.00612 & 0.00572 & 0.00586 & 0.00594 & 0.00601 & 0.00582 \\
\hline
\end{tabular}

Table 36: Energy $(\mathrm{J})$ in the 90 degree mounting orientation with $100 \%$ acceleration

\begin{tabular}{|c|c|c|c|c|c|c|c|c|}
\hline & \multicolumn{7}{|c|}{ Temperature $\left({ }^{\circ} \mathbf{F}\right)$} \\
\hline & & 80 & 90 & 100 & 110 & 120 & 130 & 140 \\
\hline \multirow{7}{*}{ 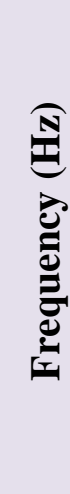 } & $\mathbf{0}$ & 0.00564 & 0.00586 & 0.00567 & 0.00589 & 0.00616 & 0.00566 & 0.00555 \\
\hline & 10 & 0.00550 & 0.00581 & 0.00571 & 0.00557 & 0.00607 & 0.00570 & 0.00550 \\
\hline & 20 & 0.00568 & 0.00602 & 0.00546 & 0.00540 & 0.00587 & 0.00568 & 0.00585 \\
\hline & 30 & 0.00571 & 0.00632 & 0.00579 & 0.00572 & 0.00586 & 0.00547 & 0.00546 \\
\hline & 40 & 0.00580 & 0.00588 & 0.00593 & 0.00577 & 0.00584 & 0.00596 & 0.00573 \\
\hline & 50 & 0.00585 & 0.00584 & 0.00588 & 0.00596 & 0.00578 & 0.00589 & 0.00591 \\
\hline & 60 & 0.00576 & 0.00585 & 0.00554 & 0.00580 & 0.00593 & 0.00605 & 0.00584 \\
\hline
\end{tabular}




\section{Focal Intensity}

Table 37: Focal intensity $\left(\mathrm{GW} / \mathrm{cm}^{2}\right)$ in the 0 degree mounting orientation with $50 \%$ acceleration

\begin{tabular}{|c|c|c|c|c|c|c|c|c|}
\hline & \multicolumn{7}{|c|}{ Temperature $\left({ }^{\circ} \mathbf{F}\right)$} \\
\hline & & 80 & 90 & 100 & 110 & 120 & 130 & 140 \\
\hline \multirow{7}{*}{ 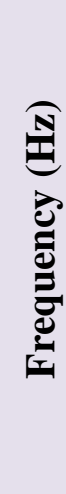 } & $\mathbf{0}$ & 106.88 & 101.86 & 100.18 & 110.67 & 118.62 & 118.99 & 114.18 \\
\hline & 10 & 103.78 & 100.26 & 99.71 & 108.92 & 115.78 & 117.55 & 113.24 \\
\hline & 20 & 105.22 & 101.42 & 98.01 & 111.33 & 117.05 & 115.90 & 110.05 \\
\hline & 30 & 109.91 & 102.45 & 101.08 & 110.60 & 123.80 & 127.80 & 115.19 \\
\hline & 40 & 106.07 & 102.68 & 101.47 & 117.16 & 129.84 & 130.36 & 114.60 \\
\hline & 50 & 109.16 & 100.94 & 98.41 & 122.60 & 131.04 & 126.57 & 121.67 \\
\hline & 60 & 112.23 & 99.74 & 102.84 & 122.98 & 129.77 & 134.24 & 123.84 \\
\hline
\end{tabular}

Table 38: Focal intensity $\left(\mathrm{GW} / \mathrm{cm}^{2}\right)$ in the 0 degree mounting orientation with $100 \%$ acceleration

\begin{tabular}{|c|c|c|c|c|c|c|c|c|}
\hline & \multicolumn{7}{|c|}{ Temperature $\left({ }^{\circ} \mathbf{F}\right)$} \\
\hline & & 80 & 90 & 100 & 110 & 120 & 130 & 140 \\
\hline \multirow{7}{*}{ 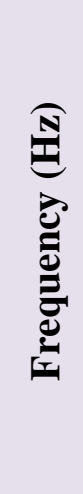 } & $\mathbf{0}$ & 106.08 & 100.43 & 99.75 & 110.68 & 120.12 & 123.00 & 117.48 \\
\hline & 10 & 103.68 & 98.40 & 102.18 & 108.59 & 115.14 & 123.80 & 111.31 \\
\hline & 20 & 107.45 & 98.96 & 99.25 & 114.02 & 98.69 & 125.31 & 115.91 \\
\hline & 30 & 103.70 & 103.66 & 99.41 & 111.64 & 123.20 & 123.02 & 113.10 \\
\hline & 40 & 104.11 & 100.95 & 98.83 & 119.06 & 121.27 & 130.09 & 114.35 \\
\hline & 50 & 105.46 & 100.09 & 100.36 & 122.12 & 126.79 & 128.66 & 120.44 \\
\hline & 60 & 105.46 & 100.20 & 103.20 & 124.64 & 125.89 & 127.68 & 123.14 \\
\hline
\end{tabular}


Table 39: Focal intensity $\left(\mathrm{GW} / \mathrm{cm}^{2}\right)$ in the 45 degree mounting orientation with $50 \%$ acceleration

\begin{tabular}{|c|c|c|c|c|c|c|c|c|}
\hline & \multicolumn{7}{|c|}{ Temperature $\left({ }^{\circ} \mathbf{F}\right)$} \\
\hline & & 80 & 90 & 100 & 110 & 120 & 130 & 140 \\
\hline \multirow{7}{*}{ 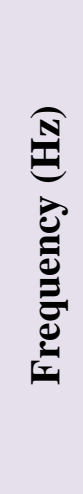 } & 0 & 105.62 & 101.57 & 107.43 & 113.84 & 117.13 & 109.56 & 101.86 \\
\hline & 10 & 104.93 & 99.97 & 106.98 & 113.48 & 116.47 & 109.03 & 102.22 \\
\hline & 20 & 106.71 & 109.86 & 106.53 & 112.11 & 111.68 & 107.78 & 102.78 \\
\hline & 30 & 103.09 & 109.93 & 110.42 & 110.29 & 109.70 & 106.40 & 103.89 \\
\hline & 40 & 111.17 & 105.98 & 112.58 & 111.80 & 109.57 & 108.56 & 105.23 \\
\hline & 50 & 110.35 & 107.76 & 110.58 & 115.29 & 110.86 & 109.91 & 104.14 \\
\hline & 60 & 109.88 & 106.34 & 110.04 & 115.07 & 113.28 & 111.66 & 104.60 \\
\hline
\end{tabular}

Table 40: Focal intensity $\left(\mathrm{GW} / \mathrm{cm}^{2}\right)$ in the 45 degree mounting orientation with $100 \%$ acceleration

\begin{tabular}{|c|c|c|c|c|c|c|c|c|}
\hline & \multicolumn{7}{|c|}{ Temperature $\left({ }^{\circ} \mathbf{F}\right)$} \\
\hline & & 80 & 90 & 100 & 110 & 120 & 130 & 140 \\
\hline \multirow{7}{*}{ 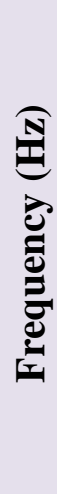 } & $\mathbf{0}$ & 104.45 & 101.15 & 107.44 & 114.60 & 115.29 & 107.89 & 101.10 \\
\hline & 10 & 102.26 & 100.19 & 105.55 & 114.20 & 118.11 & 105.64 & 100.40 \\
\hline & 20 & 105.58 & 109.61 & 106.15 & 111.82 & 111.49 & 107.02 & 102.76 \\
\hline & 30 & 103.79 & 110.86 & 112.40 & 114.43 & 113.41 & 106.30 & 106.43 \\
\hline & 40 & 108.96 & 105.43 & 111.27 & 112.31 & 108.48 & 108.46 & 104.02 \\
\hline & 50 & 112.03 & 103.84 & 111.67 & 119.16 & 112.32 & 108.83 & 105.11 \\
\hline & 60 & 108.24 & 104.77 & 110.21 & 115.15 & 112.30 & 110.57 & 104.12 \\
\hline
\end{tabular}


Table 41: Focal intensity $\left(\mathrm{GW} / \mathrm{cm}^{2}\right)$ in the 90 degree mounting orientation with $50 \%$ acceleration

\begin{tabular}{|c|c|c|c|c|c|c|c|c|}
\hline & \multicolumn{7}{|c|}{ Temperature $\left({ }^{\circ} \mathbf{F}\right)$} \\
\hline & & 80 & 90 & 100 & 110 & 120 & 130 & 140 \\
\hline \multirow{7}{*}{ 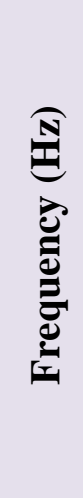 } & 0 & 101.38 & 104.51 & 105.09 & 107.11 & 107.92 & 108.79 & 109.69 \\
\hline & 10 & 100.41 & 103.83 & 104.54 & 105.81 & 108.22 & 108.23 & 107.87 \\
\hline & 20 & 103.66 & 114.41 & 104.68 & 102.75 & 105.00 & 106.24 & 108.71 \\
\hline & 30 & 103.19 & 113.51 & 107.35 & 104.71 & 104.83 & 104.63 & 108.40 \\
\hline & 40 & 105.46 & 108.67 & 107.34 & 105.62 & 106.57 & 110.53 & 108.98 \\
\hline & 50 & 107.62 & 106.46 & 107.58 & 108.79 & 105.36 & 107.79 & 109.95 \\
\hline & 60 & 104.14 & 110.66 & 104.90 & 105.94 & 106.41 & 110.43 & 108.73 \\
\hline
\end{tabular}

Table 42: Focal intensity $\left(\mathrm{GW} / \mathrm{cm}^{2}\right)$ in the 90 degree mounting orientation with $100 \%$ acceleration

\begin{tabular}{|c|c|c|c|c|c|c|c|c|}
\hline & \multicolumn{7}{|c|}{ Temperature $\left({ }^{\circ} \mathrm{F}\right)$} \\
\hline & & 80 & 90 & 100 & 110 & 120 & 130 & 140 \\
\hline \multirow{7}{*}{ 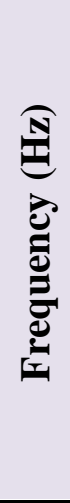 } & 0 & 101.71 & 105.77 & 102.25 & 106.25 & 111.16 & 102.17 & 100.09 \\
\hline & 10 & 99.34 & 104.82 & 103.02 & 100.54 & 109.48 & 102.89 & 99.19 \\
\hline & 20 & 102.56 & 108.68 & 98.50 & 97.49 & 105.99 & 102.47 & 105.54 \\
\hline & 30 & 103.05 & 114.05 & 104.57 & 103.28 & 105.81 & 98.71 & 98.45 \\
\hline & 40 & 104.75 & 106.08 & 106.93 & 104.21 & 105.45 & 107.62 & 103.37 \\
\hline & 50 & 105.63 & 105.46 & 106.07 & 107.52 & 104.34 & 106.37 & 106.58 \\
\hline & 60 & 103.94 & 105.53 & 99.96 & 104.64 & 107.00 & 109.19 & 105.38 \\
\hline
\end{tabular}

